\title{
EFFECTIVE CONDUCTIVITY AND THE IMPACT OF AGING EFFECTS ON FOAM INSULATIONS IN COLD CLIMATES
}

\author{
By
}

Douglas Belanger, B.Arch.Sci, Ryerson University, 2014

\author{
A thesis \\ presented to Ryerson University \\ in partial fulfillment of the \\ requirements for the degree of \\ Master of Applied Science \\ in the program of \\ Building Science \\ Toronto, Ontario, Canada, 2018 \\ (C) Douglas Belanger, 2018
}




\section{AUTHOR'S DECLARATION FOR ELECTRONIC SUBMISSION OF A THESIS}

I hereby declare that I am the sole author of this thesis. This is a true copy of the thesis, including any required final revisions, as accepted by my examiners.

I authorize Ryerson University to lend this thesis to other institutions or individuals for the purpose of scholarly research.

I further authorize Ryerson University to reproduce this thesis by photocopying or by other means, in total or in part, at the request of other institutions or individuals for the purpose of scholarly research. I understand that my thesis may be made electronically available to the public. 


\title{
EFFECTIVE CONDUCTIVITY AND THE IMPACT OF AGING EFFECTS \\ ON FOAM INSULATIONS IN COLD CLIMATES
}

\author{
Douglas Belanger \\ Master of Applied Science, Building Science, 2018 \\ Ryerson University
}

\begin{abstract}
Understanding material thermal conductivity is fundamental in high performance building design. This property is often advertised using a single value implied to be constant, though research shows that insulating materials have an effective conductivity that changes over a range of environmental parameters, including temperature and moisture levels. Various polyurethane and polyisocyanurate materials are analyzed in order to determine how the effective conductivity is altered by accelerated aging, obtained through exposure to high temperature, moisture, and freeze-thaw cycling. The measured results are used in hygrothermal simulations to determine the assumed and actual performance of insulating materials in the context of high performance wall and roof assemblies in cold climates. Results show that effects of aging and environmental temperature have higher impacts on the performance of polyisocyanurate materials than polyurethanes. Additionally, high moisture levels contribute to lower performance in all foam materials, with open cell materials experiencing the greatest performance reduction.
\end{abstract}




\section{ACKNOWLEDGEMENTS}

First, I would like to thank Dr. Umberto Berardi of the Building Science program at Ryerson University, my thesis advisor, for the tremendous help he has provided throughout the process of this research. Umberto provided great insights, helping to direct and shape the thesis. His passion for this research was instilled into me, and his encouragement kept me motivated through tough times.

I would also like to thank the other members of my thesis committee, Miljana Horvat and Russell Richman, for the valuable feedback they provided after progress reviews and the final thesis defense. I extend these thanks to the entire faculty of Ryerson University's Department of Architectural Science, specifically in the Building Science program. Many of these professors and staff have had a profound impact on my academic growth and journey through both undergraduate and graduate studies.

I would like to thank the Department of Architectural Science workshop and lab staff who aided in the design, preparation and construction of equipment and materials for this thesis work. Thank you to Greg Labbe, Frank Bowen, Jordan So and Jason Ramelson for their support in the architecture workshop and building science lab.

I wish to thank the Ontario Center for Excellence through the OCE-VIP 1 Program, the Natural Sciences and Engineering Research Council of Canada (NSERC) and the Ontario Ministry of Research, Innovation and Science through the Early Researcher Award program for their financial support, and the industry collaborators and partners for their kind donations of the tested materials.

Finally I thank my family for their unwavering support throughout my time at Ryerson University. Especially my wonderful fiancée, Kelsey, who helped keep me organized and motivated me through hard times.

To all of these people and any others who have helped me along the way, thank you. 


\section{TABLE OF CONTENTS}

Abstract $\quad$ iii

$\begin{array}{ll}\text { List of Tables } & \text { vii }\end{array}$

List of Figures $\quad$ viii

List of Appendicies $\quad$ xii

Part 1: Introduction 1

1.1 Objectives \& Research Output _. . . . . 4

Part 2: Literature Review $\quad 5$

2.1 Effective Conductivity

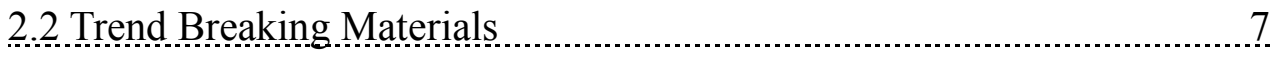

2.2.1 Polyurethane

2.2.2 Polyisocyanurate

2.2.3 Blowing Agents

2.3 Material Aging

2.3.1 Long Term Thermal Resistance (LTTR) _. 20

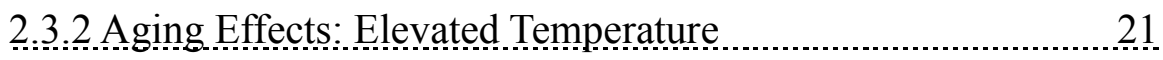

2.3.3 Aging Effects: High Moisture 23

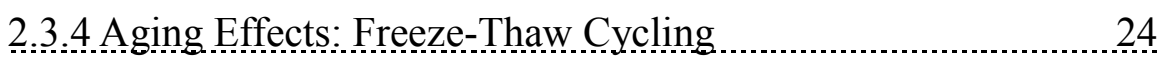

\begin{tabular}{lr} 
Part 3: Methodology & 27 \\
\hline
\end{tabular}

3.1 Materials

3.2 Temperature Dependent Conductivity $\ldots$

3.3 Moisture Dependent Conductivity $\ldots 33$

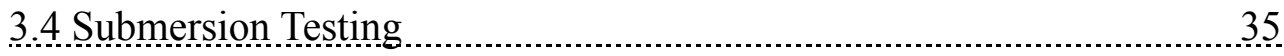

3.5 Freeze-Thaw Cycling

3.6 Elevated Temperature Aging 
4.1 Temperature Dependent Conductivity $\ldots$

4.2 Elevated Temperature Results

4.3 Moisture Exposure Results

4.4 Freeze-Thaw Results

\begin{tabular}{ll} 
Part 5: Discussion & 62 \\
\hline
\end{tabular}

5.1 Aging Analysis

5.2 Hygrothermal Simulation 70

5.3 Effective Conductivity Surface Meshes _.

5.4 Effective Conductivity Surface Models and Simulation Analysis $\quad 89$

Part 6: Conclusions 104

\begin{tabular}{ll} 
Part 7: Limitations and Future Work & 106 \\
\hline
\end{tabular}

\begin{tabular}{ll} 
Appendix & 108 \\
\hline
\end{tabular}

Bibliography 120 


\section{LIST OF TABLES}

Part 3: Methodology

3.1 Investigated Material Properties $\quad 30$

Part 4: Results

4.1 Material Mass at Varying RH\% Levels

4.2 Material Mass at Varying RH\% Levels After Freeze-Thaw 59 


\section{LIST OF FIGURES}

Part 2: Literature Review

2.1: Thermal Conductivity vs. Material Temperature 7

2.2: Thermal Conductivity vs. Moisture Content 8

2.3: Effective Conductivity of Polyisocyanurate and Stone Wool 9

2.4: Thermal Conductivity vs. Material Temperature (Polyurethane Foam) 11

2.5: Advertised, Assumed Linear and Lab Measured Temperature Dependent

Conductivity 12

2.6: Typical Aging Curve of Insulating Plastic Foam 18

2.7: Aging test of fresh polyester material at three temperatures 21

2.8: Average Ratio of Wet vs. Dry Conductivity for Common Insulating

Materials 23

Part 3: Methodology

3.1: Investigated Material Samples 29

3.2: Custom Built Environmental Chamber 34

3.3: Custom Built Submersion Tanks

3.4: Freeze-Thaw Chamber 36

3.5: Elevated Temperature Aging Oven 38

Part 4: Results

4.1: Initial Temperature Dependent Conductivity of Polyurethane A 40

4.2: Initial Temperature Dependent Conductivity of Polyurethane B 41

4.3: Initial Temperature Dependent Conductivity of Polyurethane C 41

4.4: Initial Temperature Dependent Conductivity of Polyurethane D 42

4.5: Initial Temperature Dependent Conductivity of Polyisocyanurate A

4.6: Initial Temperature Dependent Conductivity of Polyisocyanurate B 43

4.7: Measured Temperature Dependent Conductivity for All Materials $\quad 44$

4.8: Effective Conductivity of Aged Closed Cell Polyurethane (PU-A) 46

4.9: Effective Conductivity of Aged Closed Cell Polyurethane (PU-B) 47

4.10: Effective Conductivity of Aged Open Cell Polyurethane (PU-C) 47

4.11: Effective Conductivity of Aged Open Cell Polyurethane (PU-D) 48

4.12: Effective Conductivity of Aged Closed Cell Polyisocyanurate (PI-A) 49

4.13: Effective Conductivity of Aged Closed Cell Polyisocyanurate (PI-B) 49 
4.14: Effective Conductivity of Closed Cell Polyurethane A across a range of moisture levels

4.15: Effective Conductivity of Closed Cell Polyurethane B across a range of moisture levels

4.16: Effective Conductivity of Open Cell Polyurethane $\mathrm{C}$ across a range of moisture levels

4.17: Effective Conductivity of Open Cell Polyurethane D across a range of moisture levels

4.18: Effective Conductivity of Closed Cell Polyisocyanurate A across a range of moisture levels

4.19: Effective Conductivity of Closed Cell Polyisocyanurate B across a range of moisture levels

4.20: Effective Conductivity of Closed Cell Polyurethane after Freeze-Thaw

Cycles (PU-A) 56

4.21: Effective Conductivity of Closed Cell Polyurethane after Freeze-Thaw Cycles (PU-B) 56

4.22: Effective Conductivity of Open Cell Polyurethane after Freeze-Thaw.

Cycles (PU-C) 57

4.23: Effective Conductivity of Open Cell Polyurethane after Freeze-Thaw

Cycles (PU-D)

4.24: Effective Conductivity of Closed Cell Polyisocyanurate after Freeze-Thaw

Cycles (PI-A)

4.25: Effective Conductivity of Closed Cell Polyisocyanurate after Freeze-Thaw

Cycles (PI-B) 58

4.26: Moisture Content in closed cell polyurethane over a range of moisture levels (PU-A)

4.27: Moisture Content in closed cell polyurethane over a range of moisture levels (PU-B) 60

4.28: Moisture Content in open cell polyurethane over a range of moisture levels (PU-C)

4.29: Moisture Content in open cell polyurethane over a range of moisture levels (PU-D) 
4.30: Moisture Content in closed cell polyisocyanurate over a range of moisture levels (PI-A)

4.31: Moisture Content in closed cell polyisocyanurate over a range of moisture levels (PI-B)

4.32: Effective Conductivity of Closed Cell Polyurethane after 150

Freeze-Thaw Cycles and Moisture Exposure (PU-A) 63

4.33: Effective Conductivity of Closed Cell Polyurethane after 150.

Freeze-Thaw Cycles and Moisture Exposure (PU-B) 64

4.34: Effective Conductivity of Open Cell Polyurethane after 150

Freeze-Thaw Cycles and Moisture Exposure (PU-C) 64

4.35: Effective Conductivity of Open Cell Polyurethane after 150

Freeze-Thaw Cycles and Moisture Exposure (PU-D) 65

4.36: Effective Conductivity of Closed Cell Polyisocyanurate after 150

Freeze-Thaw Cycles and Moisture Exposure (PI-A) 65

4.37: Effective Conductivity of Closed Cell Polyisocyanurate after 150

Freeze-Thaw Cycles and Moisture Exposure (PI-B) 66

Part 5: Discussion

5.1: Acceleration Factors of foam materials for aging at $60^{\circ} \mathrm{C}$ 69

5.2: Basic Wood Frame Wall Assembly.

5.3: Exterior Insulated Wall Assembly

5.4: Flat Commercial Roof Assembly

5.5: Effective Conductivity for Interior Insulation Layer of Material PU-A $\quad 73$

5.6: Effective Conductivity for Exterior Insulation Layer of Material PU-A $\quad 74$

5.7: Effective Conductivity for Roof Insulation Layer of Material PU-A $\quad 75$

5.8: Effective Conductivity for Interior Insulation Layer of Material PU-D $\quad 76$

5.9: Effective Conductivity for Exterior Insulation Layer of Material PU-D 77

5.10: Effective Conductivity for Roof Insulation Layer of Material PU-D $\quad 78$

5.11: Effective Conductivity for Interior Insulation Layer of Material PI-B $\quad 79$

5.12: Effective Conductivity for Exterior Insulation Layer of Material PI-B $\quad 80$

5.13: Effective Conductivity for Roof Insulation Layer of Material PI-B $\quad 81$

5.14: Effective Conductivity (W/m*K) of Closed Cell Polyurethane across a

range of moisture levels and temperatures (PU-A). 84 
5.15: Effective Conductivity (W/m*K) of Closed Cell Polyurethane across a

range of moisture levels and temperatures (PU-B),

5.16: Effective Conductivity (W/m*K) of Open Cell Polyurethane across a

range of moisture levels and temperatures (PU-C).

85

5.17: Effective Conductivity (W/m*K) of Open Cell Polyurethane across a

range of moisture levels and temperatures (PU-D).

5.18: Effective Conductivity (W/m*K) of Closed Cell Polyisocyanurate across a

range of moisture levels and temperatures (PI-A).

87

5.19: Effective Conductivity (W/m*K) of Closed Cell Polyisocyanurate across a

range of moisture levels and temperatures (PI-B) $\quad 88$

5.20: Effective Conductivity Model for Closed Cell PU-A

5.21: Effective Conductivity Model for Closed Cell PU-B

5.22: Effective Conductivity Model for Open Cell PU-C

5.23: Effective Conductivity Model for Open Cell PU-D

5.24: Effective Conductivity Model for Closed Cell PI-A

5.25: Effective Conductivity Model for Closed Cell PI-B

5.26: Effective Conductivity for Interior Insulation Layer of Material PU-A $\quad 95$

5.27: Effective Conductivity for Exterior Insulation Layer of Material PU-A 96

5.28: Effective Conductivity for Roof Insulation Layer of Material PU-A $\quad 97$

5.29: Effective Conductivity for Interior Insulation Layer of Material PU-D $\quad 98$

5.30: Effective Conductivity for Exterior Insulation Layer of Material PU-D 99

5.31: Effective Conductivity for Roof Insulation Layer of Material PU-D $\quad 100$

5.32: Effective Conductivity for Interior Insulation Layer of Material PI-B 101

5.33: Effective Conductivity for Exterior Insulation Layer of Material PI-B 102

5.34: Effective Conductivity for Roof Insulation Layer of Material PI-B $\quad 103$ 


\section{LIST OF APPENDICIES}

8.1: Simulation (WUFI) Material Library Inputs 108

8.2: 3-D Surface Model Equations (MATLAB Outputs) 


\subsection{Introduction}

In cold climates, insulation plays an important role in the building envelope, limiting the heatflow through the wall. The performance of insulation is quantified by thermal conductivity, a property that indicates the rate of heat transfer through a given material thickness. The advertised conductivities of insulating materials are determined through lab testing in accordance with standards such as the ASTM C518 standard test method for steady-state thermal transmission properties by means of the heat flow meter apparatus (ASTM C518-15, 2015). This methodology requires that materials are tested at a mean temperature of $24^{\circ} \mathrm{C}$ using a delta temperature of $20^{\circ} \mathrm{C}$ in a heat flow meter However, research has shown that the thermal conductivity results obtained through the ASTM C518 may be misleading for determining the actual performance of building insulating materials in realistic environments. (Berardi \& Naldi, 2017). Although the ASTM C518 standard only requires measurements at one average temperature of $24^{\circ} \mathrm{C}$, it is often reported that at lower temperatures, conductivity reduces, and at high temperatures, conductivity increases. While research suggested that most fibrous insulation materials have a linear relationship between their conductivities and the temperature, in some foam insulation materials such as polyisocyanurate, the conductivity has a non-linear relationship with the environmental temperature and may experience increased conductivity at cold temperatures (Lepage \& Schumacher, 2013). This effect occurs due to the condensation of certain blowing agents within the foam cells, such as the pentane found within polyisocyanurate (Berardi, 2017). Research also shows, insulating materials have moisture content dependent conductivities in addition to temperature dependencies (Muller-Steinhagen \& Ochs, 2005). It was shown that generally, there is a linear correlation between the conductivity of a material and its moisture content. As the moisture level in the material increases, the conductivity of the material also increases due to the relatively high conductivity of water compared to the low conductivity of foam insulations. Combined, the effects of temperature and moisture levels together, determine the effective conductivity of a material, which describes the actual thermal performance of an insulating material under a given environmental condition. 
In addition to the effective conductivity of insulating materials varying over a range of temperatures and humidities, most varieties of insulation will experience a reduction in performance due to the effects of aging and environmental weathering over time. (Stovall, 2012) In foam insulation, the main cause of reduced thermal performance over time is that the effects of weathering and aging cause blowing agent gasses used in material production to escape from the foam cell structure and become replaced with air (Shukla, 2017). In regard to the aging of insulation materials, the CAN/ULCS770-15 Standard test method for determination of long-term thermal resistance of closed-cell thermal insulating foams describes the long-term thermal resistance (LTTR) to be the conductivity value measured in accordance with ASTM C518 after an aging period of 5 years in a consistent lab environment. In order to expedite the testing process, methods for accelerated aging have been developed in order to determine the LTTR on a shorter timeline (CAN/ULC-S770-15, 2015). While aging alone will have an effect on the performance of insulating materials, environmental weathering due to the effects of elevated temperature and moisture levels have been shown to contribute to the deterioration of polymer materials (Ludwick et al, 2008). Though the scope of Ludwick's research focused on the structural performance of the foams and did not include investigating the aging effect on thermal conductivity, it will be valuable to investigate the effects that these types of aging techniques have on the conductivity of the materials. Research regarding foam materials undergoing freeze-thaw cycling has also shown that the moisture storage capacity of foam materials including closed-cell insulation will increase after $\sim 165$ cycles (Garber-Slaght, 2012). This indicates that in a cold climate where freeze-thaw cycles are common, aged materials may have increased ability to hold moisture, and therefore have the potential to experience larger impacts on performance from high moisture levels, in addition to any other physical degradation that the freeze-thaw cycles may cause to the material.

Investigation into the performance of insulating materials over a range of temperatures and humidities, as well as how those relationships are affected by the aging of the material will provide valuable insight into the expected performance of these materials in the built environment. As the 
building industry moves towards high efficiency design strategies such as Passive House, not only the level of performance, but also the accuracy at which the performance of insulation can be determined will greatly impact the functionality of the design as a whole. More thorough understanding of foam insulation materials will assist designers in the development of efficiently designed assemblies that will perform at a level similar to what is assumed prior to construction. 


\subsection{Objectives \& Research Output}

This study explores the effects of environmental weathering and aging, through exposure to high temperature, moisture, and freeze/thaw cycles, on the temperature and humidity dependent conductivity of foam insulating materials. Using this information, hygrothermal models are developed to assess how insulation materials perform over the life cycle of a building under real environmental conditions. These developments should inform designers about the dynamic nature of material conductivity and promote informed design, taking into account the temperature dependent conductivities and the effects of aging for insulating materials being used in envelope design.

Over the course of this research project, two major outputs are developed. The first major output will take the measurements that indicate conductivity of the materials over a range of temperatures and moisture levels, developing three-axis plots to illustrate the effective conductivity of the selected materials as a function of both temperature and moisture level. Additionally, the effect of various aging processes on the effective conductivities will be measured. This data provides insight into the potential range of effective conductivity a material may experience, and will be used for further analysis of the materials within the context of a building envelope.

The second output will be the results of hygrothermal simulations for materials simulated in various wall and roof assemblies in a Toronto, ON climate. This output allows for further analysis regarding which materials are better suited to be used under certain environmental conditions or assembly designs. The results of this study will allow designers to further understand the dynamic nature of the foam insulation materials that were analyzed and how their effective performances may change over the life-cycle of a building. Valuable information regarding the true thermal performance of insulating materials under realistic environmental conditions can lead to more accurately designed building envelope assemblies that aim to meet specific performance benchmarks. 


\subsection{Literature Review}

In this literature review, topics related to the study of temperature and relative humidity dependent conductivity will be discussed. The concepts of temperature dependent and moisture dependent conductivity will be explored, and an understanding of what causes the phenomenon to occur will be developed. Research regarding the material properties of foam insulating materials will also be reviewed in order to gain a deeper knowledge of the materials themselves, as well as beginning to understand how various materials are affected in different ways by temperature and relative humidity. With the focus on foam insulation, elements pertaining to foams such as the effect of blowing agents will also be discussed. Effects of aging and environmental conditions on the performance of foam materials will be discussed, in order to develop an idea of the knowledge gaps or uncertainties in current studies. The methodologies and results of similar research projects will be discussed, providing a useful framework for the development of the methodology for this research project, as well as an understanding of the state of knowledge in the area of aging effects on foam insulating materials. 


\subsection{Effective Conductivity}

In order to understand the impact of temperature and moisture on the conductivity of insulating materials, first the concept of temperature dependent conductivity must be understood. A common misconception regarding insulation materials is that the conductivity of a given material is constant over a range of environmental conditions. Advertised conductivity values for insulation are based on testing at $24^{\circ} \mathrm{C}$ in accordance with ASTM C518 - 15 while the advertised long term thermal resistance (LTTR) is determined under strictly controlled laboratory settings such as the method described in CAN/ULC-S770-15 which is used in the case of closed-cell foam insulation. Under the standards for determining the LTTR of a material, the thermal conductivity value is still based on measurements in accordance with ASTM C518 - 15 at $24^{\circ} \mathrm{C}$.

Research conducted by Grin et. al mention that for most materials, there tends to be a linear trend for conductivities. At cold temperatures conductivities are generally lower, where at higher temperatures the conductivities are higher (Muller-Steinhagen \& Ochs, 2005). In Fig. 2.1, this change in conductivity is shown for various insulating materials. Temperature dependent conductivity can be explained through basic state of matter laws in physics. As the temperature of a material increases, the molecules of the material will vibrate and move faster, allowing for increased rates of heat transfer through conduction. 


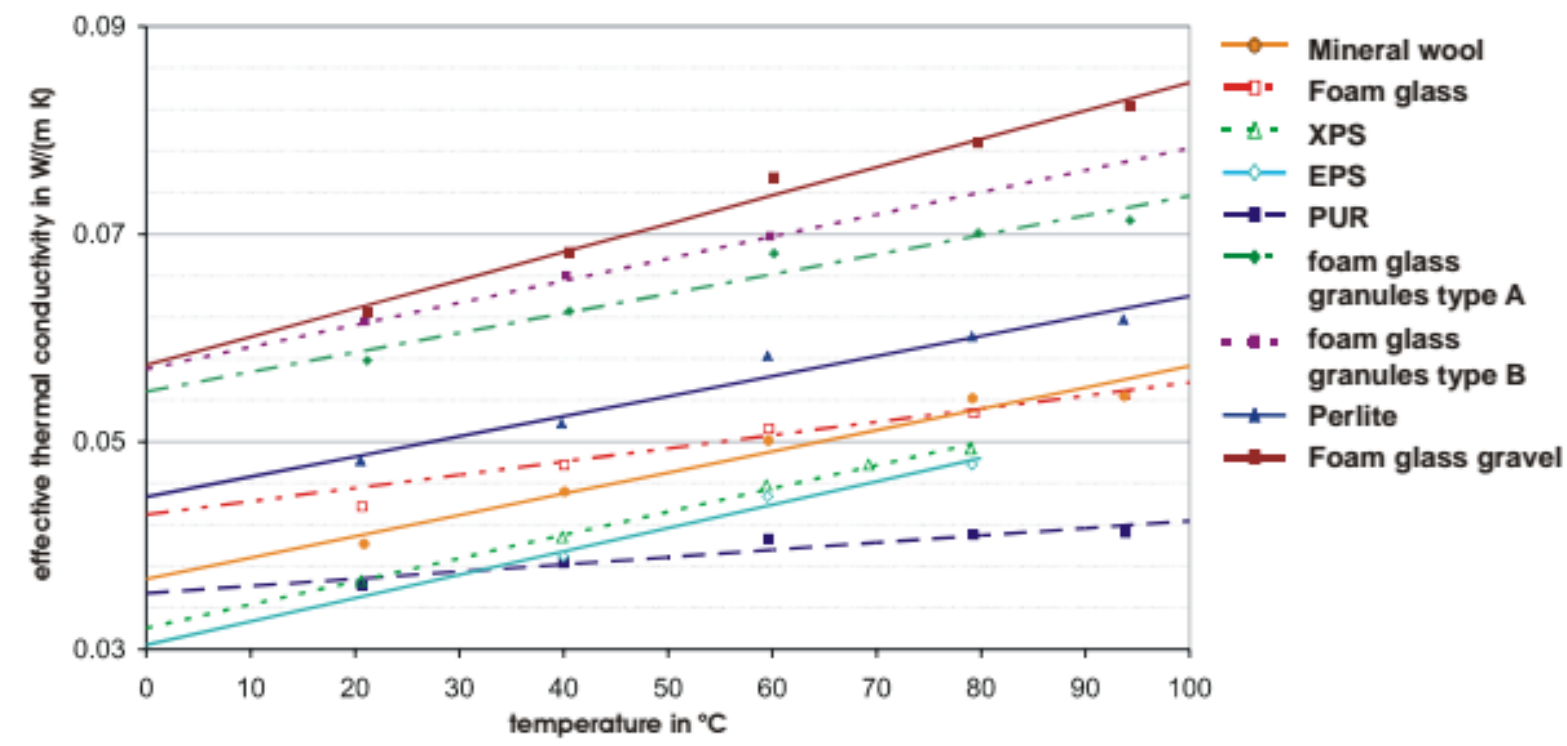

Figure 2.1: Thermal Conductivity vs. Material Temperature (Muller-Steinhagen \& Ochs, 2005)

Similarly, according to Muller-Steinhagen \& Ochs (2005), diffusion into insulating materials due to humidity causes an increase in thermal conductivity as the moisture content increases. As shown in Fig. 2.2, as more the level of moisture increases, the conductivity of the material also increases due to the relatively high conductivity of water $(\sim 0.6 \mathrm{~W} / \mathrm{mK})$ compared to the conductivity of dry foam insulation which are generally advertised to perform in the range of $\sim 0.02-0.04 \mathrm{~W} / \mathrm{mK}$. Additionally, the results show that at higher temperatures, the rate at which the increasing moisture content impacts the conductivity is higher (Muller-Steinhagen \& Ochs, 2005). 


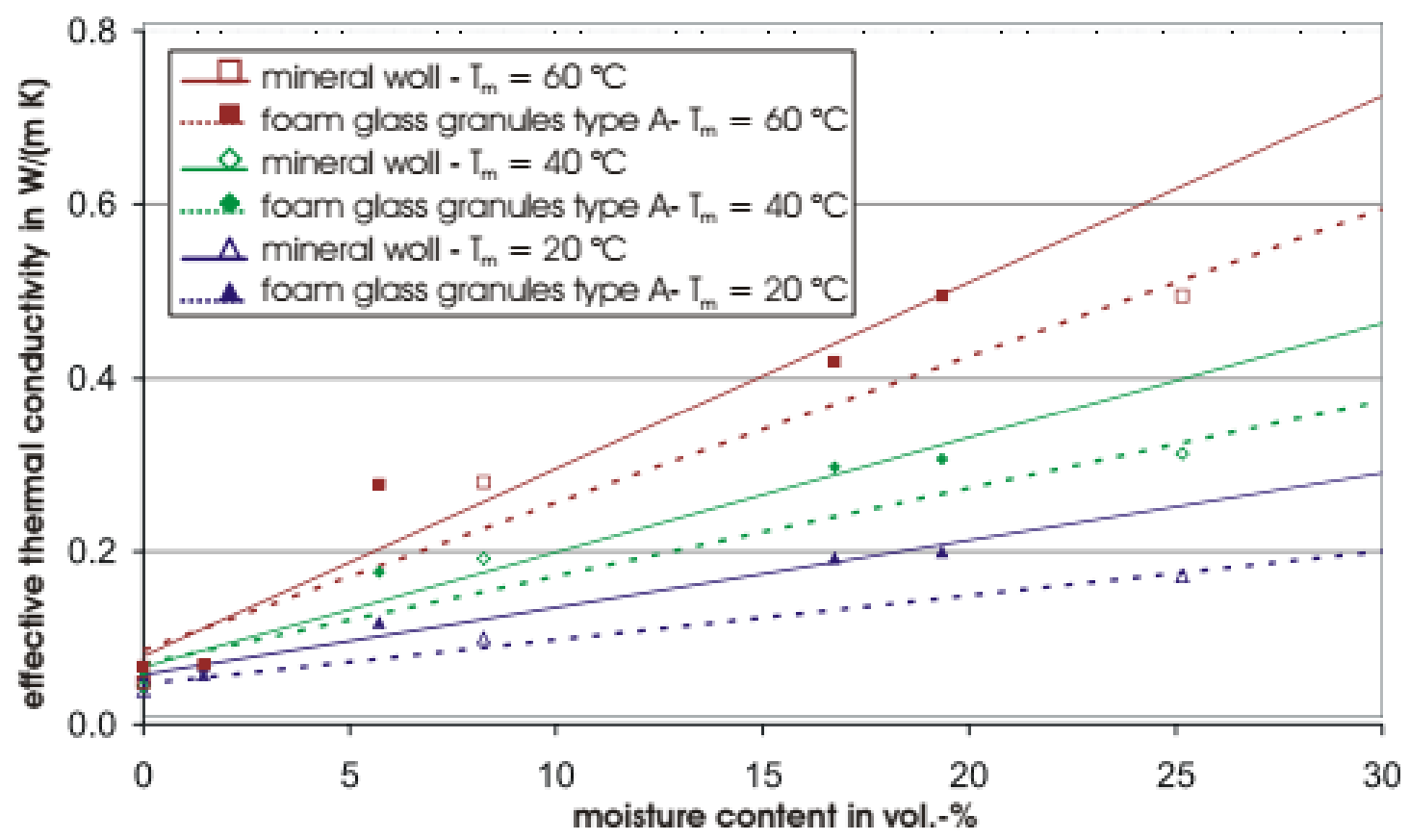

Figure 2.2: Thermal conductivity vs. moisture content (Muller-Steinhagen \& Ochs, 2005).

Between the impact of both, temperature dependent and moisture content dependent conductivity, the overall thermal resistance of a wall or roof assembly that was designed under the assumption of a constant conductivity may perform differently than expected. In many modern hygrothermal simulation softwares, material databases often make use of an assumed linear temperature dependent conductivity in an attempt to increase accuracy (Lepage \& Schumacher, 2013). Alternate standards have started to acknowledge this effect and suggest that the reported thermal properties of materials should be measured under conditions closer to the final applications in order to more accurately assess the performance of a material in its intended environment (ASTM C1058). This issue is often important in cases of insulation for pipes which carry liquids at very high or low temperatures such as boiled water or refrigerants below freezing temperatures, but further research suggests that the range of temperatures experienced in the exterior environment can have large impacts on performance as well. 


\subsection{Trend Breaking Materials}

While an assumed linear temperature dependent conductivity does provide an adequate model for most materials, some materials do not follow a linear trend. Some foamed insulation products such as polyurethane and polyisocyanurate appear to have some unusual behaviour in certain temperature ranges (Lepage \& Schumacher, 2013). In Fig. 2.3, the measured thermal conductivity of polyisocyanurate is compared to a stone wool insulation board. As seen in the figure, the stone wool follows a linear temperature dependent conductivity, while the conductivity of Polyisocyanurate (PIC) seems to suddenly increase as the temperature falls below $15^{\circ} \mathrm{C}$ (Grin at al., 2014).

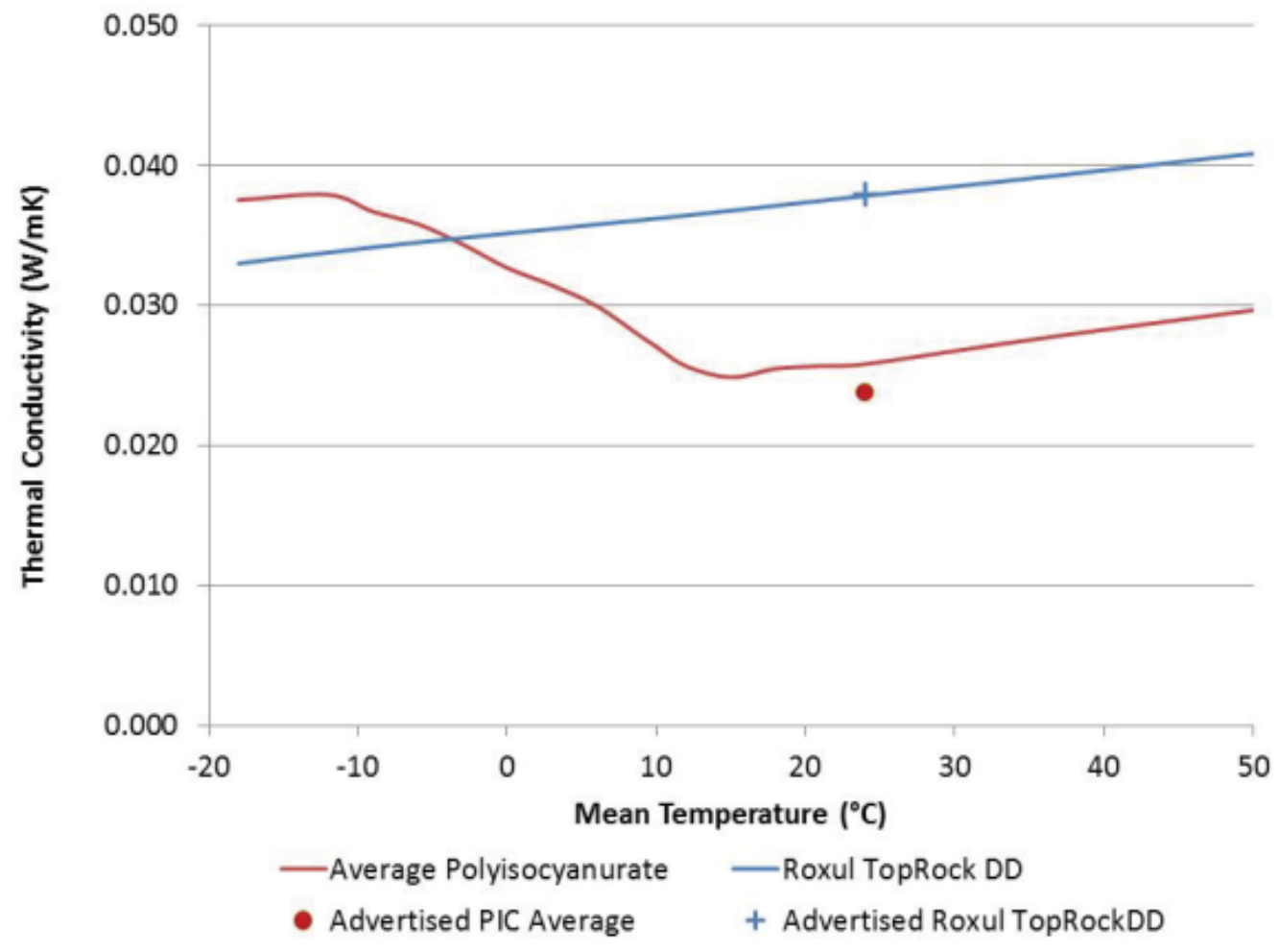

Figure 2.3: Effective Conductivity of Polyisocyanurate and Stone Wool (Grin et al., 2014) 
The study conducted by Grin et al (2014), reports that in simulations of a large flat roof taking into account the measured conductivity of the polyisocyanurate material, it was calculated that the conductivity of polyisocyanurate could be in the range of $32-58 \%$ higher in the heating season and 16 $37 \%$ higher during the cooling season compared to its advertised value (Grin et al, 2014). In a similar study, Lepage \& Schumacher (2013), found similar results when assessing a range of polyisocyanurate materials. In the analysis of the results in these studies, it is not mentioned in detail the reasons that this type of effect may be occurring in certain insulating materials, but rather just that the effect was occurring.

A study of the temperature dependent thermal conductivity of polyurethane foam conducted by Tseng et al, (1997), provides deeper analysis at the material level regarding its unusual thermal behaviour. In the study, the conductivity of polyurethane foam is explored both theoretically and through measurements from $20 \mathrm{~K}\left(-253^{\circ} \mathrm{C}\right)$ to $300 \mathrm{~K}\left(27^{\circ} \mathrm{C}\right)$ (Tseng et al., 1997). The report analyzes the heat transfer mechanisms in polyurethane to high levels of detail, and provides insight into the nonlinear trend found in the temperature dependent conductivity for both polyurethane and polyisocyanurate. Shown below, in Fig. 2.4, are the measured and theoretical conductivities of polyurethane foam in a temperature ranger from $20 \mathrm{~K}\left(-253^{\circ} \mathrm{C}\right)$ to $300 \mathrm{~K}\left(27^{\circ} \mathrm{C}\right)$. Similarly to what is seen in Fig 2.3, the polyurethane foam measured in Tseng's study (1997) also has a conductivity increase as the temperature falls, in this case, as the material begins to fall below $273\left(0^{\circ} \mathrm{C}\right)$. Interestingly, as the temperature continues to fall past $\sim 230 \mathrm{~K}\left(-43^{\circ} \mathrm{C}\right)$, the conductivity trend becomes linear again, and additionally seems to have linear gain in conductivity as the temperature increases past $\sim 270 \mathrm{~K}\left(-3^{\circ} \mathrm{C}\right)$. 


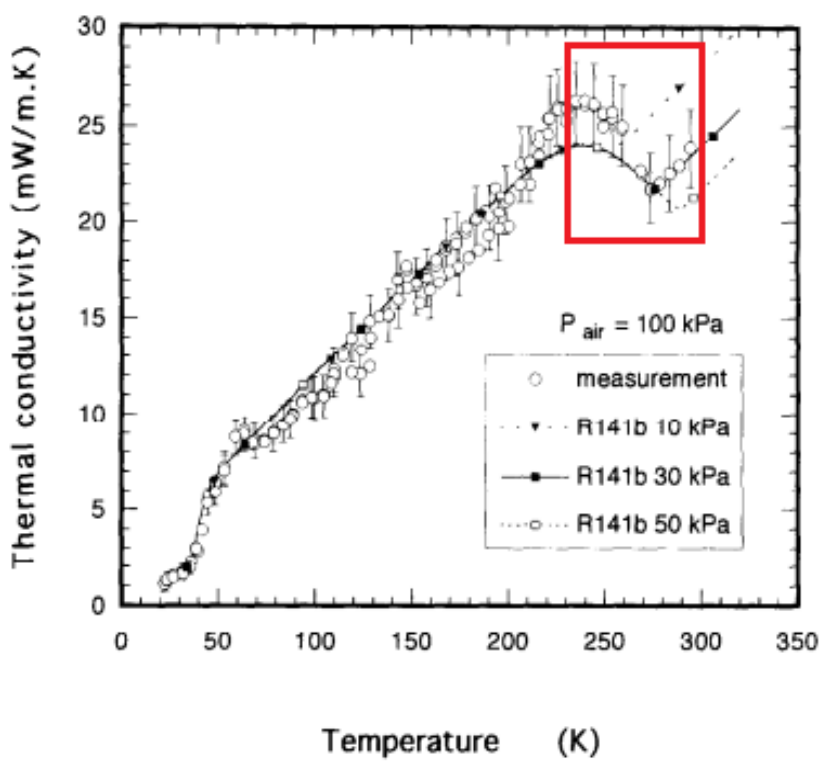

Figure 2.4: Thermal Conductivity vs. Material Temperature (Polyurethane Foam) (Tseng et al., 1997)

Tseng also explains what is happening within the material where this non-linear trend is occurring. As the temperature of the polyurethane foam decreased below 270K, the R141b gas within the material begins to condense, increasing the conductivity. At $230 \mathrm{~K}$, the gas is fully condensed, and the linear trend begins to occur again as the temperature reduces from there (Tseng et al., 1997). It is also mentioned, that due to the uncertainties in the processing conditions, blowing agents, and gasses used in polyurethane manufacturing, this trend may occur differently in any given sample. In modern production, the R141b gas is no longer used. It was discontinued in favor of the current HFC (hydrofluorocarbons) blowing agents in polyurethanes and pentane gasses in polyisocyanurate foams. Through recent studies performed by the Building Science Corporation (Grin et al, 2014) it was confirmed that some foam insulating materials such as polyisocyanurate are still experiencing the effect of increased conductivity at low temperatures, suggesting that some number of the currently used blowing agents in polyisocyanurate are experiencing similar phenomenon to that described by Tseng et al. (1997), for the polyurethane sample that was investigated. 


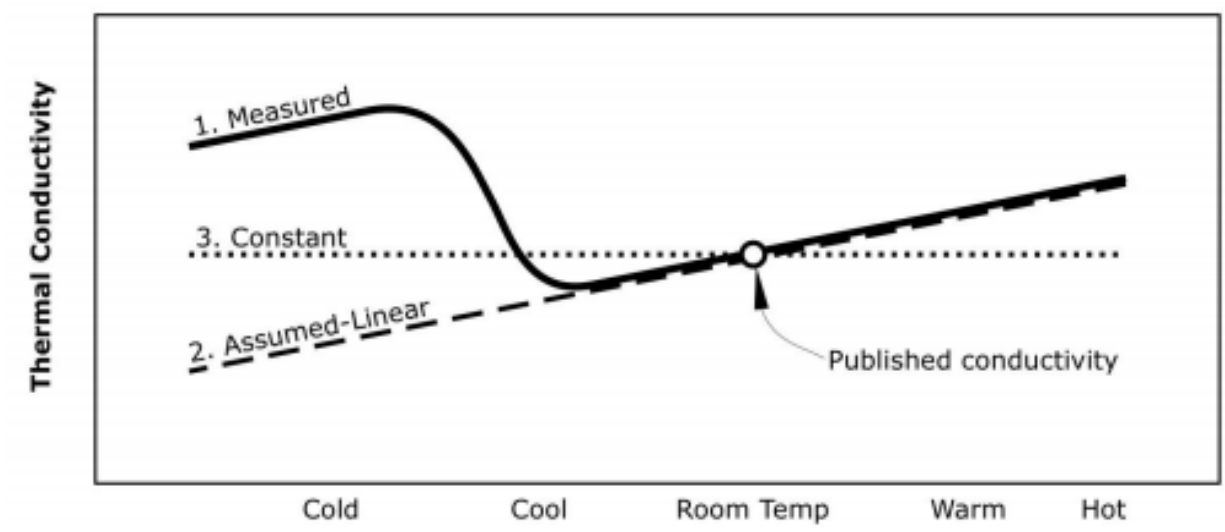

Figure 2.5: Advertised, Assumed Linear and Lab Measured Temperature Dependent Conductivity

(Lepage \& Schumacher, 2013)

Overall it is very important to understand the material properties of all insulation products that are being designed with. The difference between an advertised (constant), assumed-linear, and a true measured material conductivity may vary greatly depending on the environmental condition as represented in Fig. 2.5. Especially in the case of materials that have many uncertainties in the manufacturing process, specifically in the blowing agents, such as in the cases of most polyisocyanurate and polyurethane foams, lab testing for a measured temperature and humidity dependent thermal conductivity provides more accurate information to use in envelope design.

In this study, the materials being focused on are foam based insulating materials, where studies have shown inconsistencies in the conductivity over a range of environmental conditions. Commonly used foam insulating materials include polyurethane and polyisocyanurate. Each of these materials will be studied in order to discover why they each may react to changing environmental conditions and aging effects differently. The following sections review details of these foams. 


\subsubsection{Polyurethane}

Polyurethane foam is a very versatile material, used for many purposes from insulation to padding. There are two major types of polyurethane foam, open and closed cell. Within each of these categories there are further distinctions within the types of material and their functions. The focus of this study will be on the insulating performance of polyurethane foam. The two types of insulating polyurethane that will be assessed are low density semi-rigid open cell foams, and high density rigid closed cell foams.

In open cell polyurethane, the foam is expanded using $\mathrm{CO}_{2}$ as a blowing agent, as the foam expands, bubbles within the foam develop open connections to each other allowing the $\mathrm{CO}_{2}$ to escape and be replaced by air, this means that the foam is permeable to both air and vapour. Open-cell insulation is often referred to as low density foam and generally is produced to be $8 \mathrm{~kg} / \mathrm{m} 3(0.5 \mathrm{lbs} /$ cu.ft.), and has a firm cushion structure (CUFCA, 2005). Low density open cell polyurethane foam generally provides $9.76 \mathrm{RSI} / \mathrm{m}(\sim 3.5 \mathrm{R} / \mathrm{inch})$. Closed cell polyurethane is expanded using a blowing agent such as an HFC (hydrofluorocarbon) which have a higher thermal resistance than air. Through the nature of the foam expansion with the blowing agent, a more dense foam is created and bubbles are prevented from developing open connections, leaving the gas trapped within the foam bubbles. This creates a material that has a higher thermal resistance value than an open cell foam, and is impermeable to air after 1" of thickness as well as to vapour after 2" of thickness according to refrigerant manufacturer technical data. Generally, closed cell insulations are referred to as medium or high density foam. medium density foams, (the more commonly used closed cell foam), usually have a density of $32 \mathrm{~kg} / \mathrm{m} 3$ (2lbs/cu.ft.) and provide a thermal resistance value of $18.2 \mathrm{RSI} / \mathrm{m}(\sim 6.5 \mathrm{R} / \mathrm{inch})$ (CUFCA, 2005). High density foams are generally only used where greater compressive strength is required, and can be produced to a density greater than $48 \mathrm{~kg} / \mathrm{m} 3$ (3.0lbs/cu.ft).

Research presented by CUFCA also indicates that there is a correlation between the overall thickness of 
the spray foam insulation and its long term thermal resistance per meter. They describe that the effects of off gassing and aging generally occur on the surface of the material, meaning that the thicker that the material is sprayed, the higher the overall thermal resistance over time. With the material in the center of the foam being less affected by aging, that portion of the foam holds a higher thermal resistance for longer (CUFCA, 2005). 


\subsubsection{Polyisocyanurate}

Similar in nature to polyurethane foam, polyisocyanurate is another versatile foam capable of performing as a high efficiency insulator. Polyisocyanurate products are all relatively high density closed-cell products which are manufactured similarly to polyurethane, using blowing agents to expand the polymer material into foam, currently making use of various pentane gas mixtures (Letts et. al, 2016). Polyiso is most commonly used in roofing applications for cold climates, as well as exterior insulation of an existing wall. Generally being advertised as the highest thermal resistance rating per material thickness, some of the high efficiency varieties have conductivities advertised to be $0.018-0.02$ $\mathrm{W} / \mathrm{mK}$. A major difference between polyurethane and polyisocyanurate is that polyisocyanurate is generally manufactured into boards, where most polyurethanes are sprayed in place.

polyiso is advertised with a lower thermal conductivity than other foam insulation materials, making it a common choice for large commercial/industrial roofing projects (Shukla, 2017). Similar to closedcell polyurethane, this material gains much of its thermal resistance value from the blowing agents which exist in the foam cells. In the case of polyisocyanurate, pentane gas is often the blowing agent used in the manufacturing process. While the pentane gas provides a high thermal resistance in the foam cells, it is also suggested that this gas may be the reason that polyisocyanurate foams have been found to perform worse at cold temperatures, due to the pentane blowing agent condensing at low temperatures, increasing the conductivity of the material. (Lepage, 2013) 


\subsubsection{Blowing Agents}

Blowing agents are used in the manufacturing process of foam insulation to expand the foam material through chemical reaction, provide thermal resistance within closed cell foams, and have a large impact on the end product of the material (Bogdan et al., 2011). Historically, CFC and HCFC gasses were once used as blowing agents, though due to issues of ozone damaging material and global warming potential, these blowing agents were phased out after the "ODP Reduction Montreal Protocol" in 1987, which outlawed the use of ozone depleting materials (Environment Canada, 1987). Currently, HFC (hydrofluorocarbon) gas is used as the blowing agent for most polyurethane foam insulation. While this material does not have the same ozone depleting effects of CFC or HCFC, it still has a very high global warming potential and is currently being phased out. HFO (hydrofluoroolefin) gas represents the next generation of available blowing agents. According to manufacturers, this gas provides equal or better performance than HFC gasses while having a much lower global warming potential (Bogdan et al. 2011). Using HFO gas will mean that over time, off gassing from the foam insulation materials may not be as big of an issue as with HFC or older blowing agents in terms of their impact on the ozone layer as well as reducing contribution to global warming. Unfortunately, research performed on materials using HFO blowing agents have not been carried out at an extensive level, and there is a gap in knowledge regarding performance of these materials, especially considering effective conductivity.

After the phase out of HCFCs, most polyisocyanurate manufacturers moved toward Pentane gas mixtures as blowing agents (Letts et. al, 2016). As evidenced by recent research carried out by Grin et al (2014), as well as Letts et al (2016), polyisocyanurate materials are currently the focus of materials that do not conform to the assumed linear trend in temperature dependent conductivity. While polyurethane materials have shown this effect in past studies (Tseng et al., 1997), more recent investigations reveal that the occurrence is more prevalent in polyisocyanurate samples. Research 
suggests that this may be related to the changes in blowing agents used between the date of Tseng's 1997 investigation and the investigations by Grin et al., in the last 5 years (since 2013), where polyurethanes moved towards HFC's where Polyisocyanurates moved to Pentane gasses. As blowing agents evolve, it is important to revisit how the new materials behave as a result of aging effects, as investigations of newer foam materials are lacking. 


\subsection{Effects of Material Aging}

As the effects of effective conductivity in foam insulations are explored, the issue of aging effects on effective conductivity becomes very important as well. Over time, foam insulation materials experience three stages throughout their aging process. The effect of aging in foam materials is seen in both closed cell polyurethane and polyisocyanurate materials. One of the most impactful aging effects on the thermal conductivity is the diffusion of the highly insulating blowing agents, and the infusion of air from the environment which may carry moisture into the material. These two effects begin to occur immediately after the manufacturing process, but the rate at which the gas movement occurs is much faster for the infusion of outside air than for the diffusion of blowing agent gasses. It is suggested that the infiltration of exterior air takes place over the first 1-2 years of the material life cycle, while the diffusion of blowing agent gasses take place over 10-20 years (Shukla, 2017). This sheds light on the model presented in Fig. 2.6, where initially the conductivity of a foam material increases rapidly (the effect of air infiltration), and then slowly plateaus (the effect of blowing agent gasses diffusing expires). The implication of this aging model is that any given foam insulation material will perform closer to its manufactured state for a short time, while it will perform at a level closer to its final conductivity for the majority of the life cycle.

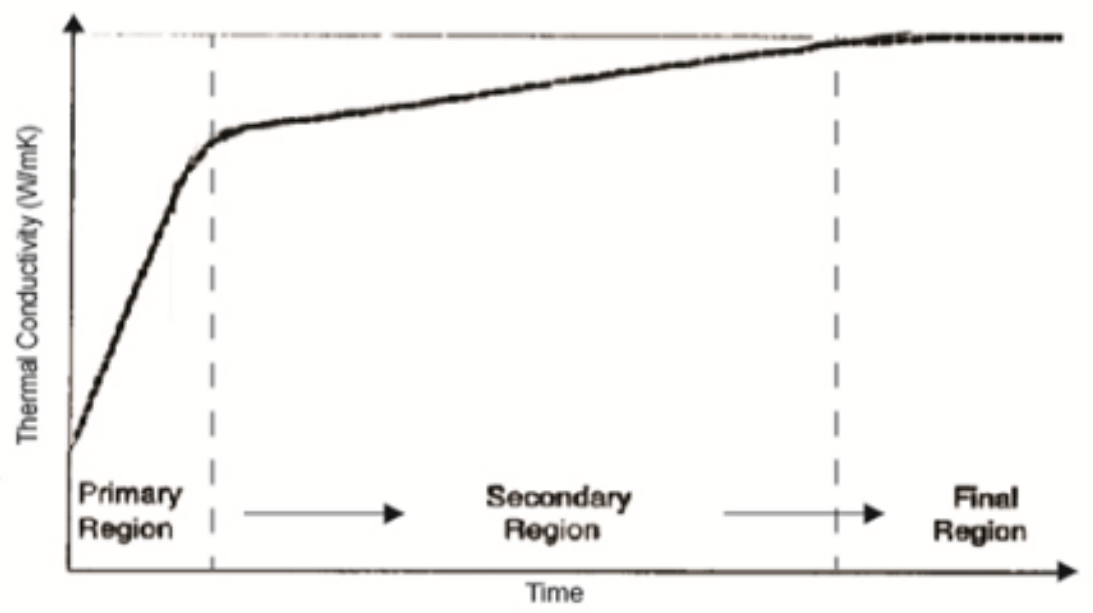

Figure 2.6: Typical Aging Curve of Insulating Plastic Foam (Bhattacharjee et al., 1994) 


\subsubsection{Long Term Thermal Resistance (LTTR)}

As the effects of material aging are explored, the final effective conductivity becomes more important. Currently, the long-term thermal resistance rating (LTTR) is the Canadian standard value used for advertising the long term thermal conductivity value of foam insulations. The LTTR of a material is described in the CAN/ULC-S770 (2015) standard as the conductivity of the material after 5year storage in a laboratory setting. The LTTR value is assumed to be close to the conductivity in the final region in the aging curve. The CAN/ULC-S770 (2015) standard also provides the framework for determining the LTTR of a closed cell foam insulation sample at an accelerated rate, through the thin slice stack method. Due to the progressively reduced thickness of the material samples, and consequently, to the higher surface area to volume ratio, the insulating blowing agent gasses are able to escape at a faster rate, and a higher percentage of the original blowing agent molecules are replaced with air. This allows the samples to undergo a simulated aging of five or more years over the course of a shorter duration based on Eq.1, where $\mathrm{t}$ is temperature and $\mathrm{L}$ is material thickness.

$$
(\mathrm{t} 1 / \mathrm{t} 2)=(\mathrm{L} 1 / \mathrm{L} 2)^{2} \quad \text { Eq. } 1-\text { LTTR Aging Factor }
$$

Currently in order to determine the Long term thermal resistance rating in foam insulations, manufacturers must perform measurements on the aged materials at $24 \mathrm{C}$. While this test method may give an indication of the thermal resistance in a lab environment after aging, the effective conductivity curve over a larger temperature range may not be affected uniformly, this greatly increases the level of uncertainty in the actual performance of foam insulation materials. Another major issue with the LTTR method is that it assumes only diffusion of the blowing agent gasses have an effect on the aged thermal conductivity of the material, and disregards environmental conditions that materials would be exposed to outside of a laboratory environment, such as varying moisture levels and temperature ranges, as well as polymer degradation over time due to freeze-thaw cycles or other environmental conditions, which 
are not reflected in the thin slice method. In particular, the polymer degradation could be simulated through heat exposition, rather than reducing material thickness through the thin slice method (Ludwick et al, 2008).

Further investigative studies have been carried out on polyurethane foams in order to determine the effects of accelerated aging on structural material properties after aging under high temperatures (Tcharkhtchi, 2014) as well as through UV exposure and moisture exposure (Ludwick et al, 2008). Though the purpose of both of these studies were to understand the structural properties of the material, the framework for these studies can easily be applied to accelerated aging in order to observe the effects that these same types of aging may have on the thermal conductivity of the materials. Due to the very different effects that the various methods of aging have on materials, it may be important to explore how materials are affected in the context of their thermal conductivity. 


\subsubsection{Aging Effects: Elevated Temperature}

The effect of material aging is an important issue that can have an impact on the insulating value of the material over time. The effects of aging occur slowly in most insulating materials, and in order to develop an understanding of the effect of aging on foam insulation, the aging process must be expedited. In a study on the aging of polyester materials, it was found that the temperature at which a material is stored at, has a large impact on the aging process. At higher temperatures, the material was found to age at a much faster rate. (Hunter et al., 2000) Though the testing in this project was not specific to insulating foams, the methodology used in the project can be applied to other materials as well. As shown in Fig. 2.7, it was found that at higher temperatures, the rate of deterioration increases substantially.

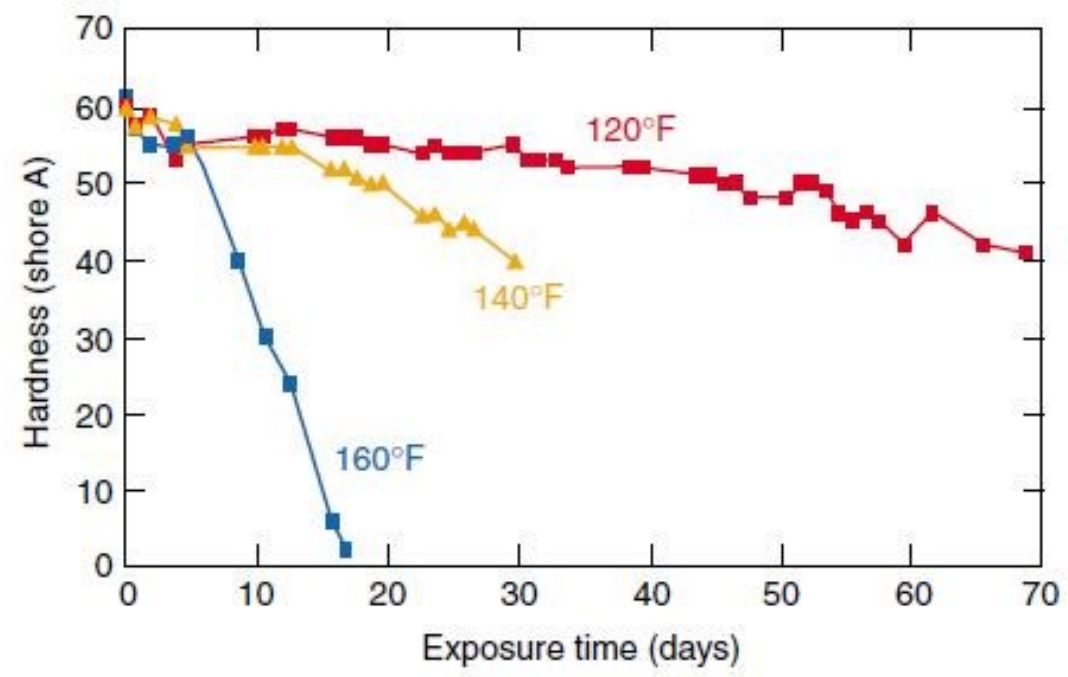

Figure 2.7: Aging test of fresh polyester material at three temperatures (Hunter et al, 2000)

As reported by Jelle (2011), accelerated aging through various mechanisms in addition to temperature, including hygrothermal exposure and UV radiation will also affect building materials in different ways over time. Jelle (2014) also reported that through using the Arrhenius Equation, the duration of the expedited aging process can be correlated to the time it takes for the material to age similarly in standard environmental conditions. Knowing this, foam insulation samples can be stored at 
higher than standard heat levels for an extended period of time, and later it can be determined the equivalent age in a standard environment, allowing conclusions to be drawn regarding the effect of long term aging on performance of foam insulation. 


\subsubsection{Aging Effects: Moisture Exposure}

High moisture levels have a negative impact on the thermal resistance of insulating materials. As moisture levels within insulation increase, the performance is reduced through the water content providing a reduced average thermal resistance in the material. Additionally, excess moisture may cause water damage in the building envelope, as well as providing environments that can encourage mold growth. As a general rule, it is important to ensure that moisture levels are adequately handled through the design of the building envelope to reduce the risk of moisture related issues occurring.

Theoretically, closed-cell materials should have their performance affected minimally by high moisture levels due to the nature of closed cell materials being water resistant. In studies investigating the long term effects of high moisture levels on foam insulation materials, it was found that over time even these closed-cell materials absorb a small amount of water. (Garber-Slaght, 2012)

\begin{tabular}{|c|c|c|c|c|}
\hline Insula ion & $\begin{array}{c}k_{d \mathrm{dy}} \\
\left(\mathrm{W} / \mathrm{m} \cdot{ }^{\circ} \mathrm{C}\right)\end{array}$ & $k_{\text {med }} / k_{d y}$ & $\begin{array}{l}\text { Fraction of total } \\
\text { installed: } 1983-88 \text { [4] }\end{array}$ & $\begin{array}{l}\text { Fraction of total } \\
\text { installed: } 1992 \text { [5] }\end{array}$ \\
\hline Polyisocyanurate & 0.021 & 1.14 & 0.28 & 0.49 \\
\hline $\begin{array}{l}\text { Expanded } \\
\text { polystyrene }\end{array}$ & 0.034 & 1.22 & 0.08 & 0.12 \\
\hline $\begin{array}{l}\text { Extruded } \\
\text { polystyrene }\end{array}$ & 0.029 & 1.27 & 0.08 & 0.06 \\
\hline Phenolic & 0.016 & 1.30 & 0.00 & 0.03 \\
\hline Polyurethane & 0.027 & 1.14 & 0.05 & 0.00 \\
\hline Wood fiber & 0.058 & 1.76 & 0.18 & 0.00 \\
\hline Perlite & 0.052 & 2.14 & 0.10 & 0.25 \\
\hline Glass fiber & 0.036 & 2.35 & 0.22 & 0.07 \\
\hline
\end{tabular}

Figure 2.8: Average Ratio of Wet vs. Dry Conductivity for Common Insulating Materials (Desjarlais \& Kyle, 1994) 
In Fig. 2.8, values regarding the dry vs. wet thermal conductivity of a selection of insulation materials including the closed-cell foam materials polyisocyanurate, polyurethane, expanded polystyrene and extruded polystyrene are shown. It can be seen that while the closed-cell materials do not experience as massive a difference between the dry and wet performance when compared to a material like glass fiber, where moisture can more freely travel into and through the material, there is still a $14 \%$ increase in conductivity for polyurethane and polyisocyanurate materials when wet.

It is very important to understand that even closed cell materials will be affected by moisture over time, especially when the material experiences many freeze/thaw cycles which can more quickly degrade a closed-cell foam material causing the maximum potential moisture content to increase. In envelope design where a high degree of accuracy regarding the material thermal properties is necessary, decreased performance over time due to wetting may have detrimental effects on the system loads for the building. 


\subsubsection{Aging Effects: Freeze-Thaw Cycling}

Investigations into the moisture issues for foam insulation revealed that freeze/thaw cycling will increase the amount of moisture that is allowed to infiltrate the material samples. (Garber, 2012) In Garber's testing, it was found that a new sample of polyurethane was able to absorb only $1.2 \%$ of its weight in moisture content and was able to dry very rapidly, after being submerged for 3 months. After 168 freeze/thaw cycles in a humid environment, the moisture content increased to $5.2 \%$ of the material weight and did not dry fully as quickly. It has also been found that as polyurethane foam materials undergo freeze-thaw cycling, more moisture is absorbed into the material when submerged (GarberSlaght, 2012). This research suggested that a foam material undergoes more freeze-thaw cycles, the polymers degrade and allow the cells to absorb more moisture. As the conductivity of a material will increase as the water content rises, the effect that freeze-thaw cycles have on a foam materials ability to absorb and store moisture may hence be very important. 


\subsection{Summary of Literature Review}

Through the research discussed in the literature review, it becomes clear that effective conductivity of insulating materials may vary from the advertised value, due to a combination of environmental conditions and aging effects. The effects of these factors on the performance of foam insulating materials, especially those which contain blowing agents (closed-cell foams), have been shown to stray from the assumed behaviour of linear temperature and moisture dependent conductivity. It will be important to continue to investigate new materials as blowing agents evolve, in order to determine whether the effective performance of the materials will vary with chemical changes. While past research has explored both temperature and moisture dependent conductivity, there is little experimental data regarding effective conductivity as a function of both temperature and moisture level. Many investigations look into the effects of aging and environmental weathering on foam materials, including elevated temperature exposure, exposure to high moisture levels, submersion in water, and freeze-thaw cycling. These investigations often focus on the structural properties of the material and provide limited results regarding the effective thermal conductivity of the material. In the case of determining the long term thermal resistance, industry standardized tests require measuring the long term thermal conductivity (LTTR) at a standard lab condition only (CAN/ULC-S770, 2015). Research suggests that this LTTR value may be a poor indication of the actual long term performance of a material, due to the standardized test methods ignoring a number of factors that affect thermal performance It is clear that more investigation into the effective performance of foam insulating materials through lab testing will provide a much more accurate understanding of how materials will perform over their life-cycle. 


\subsection{Approach \& Methodology}

The objectives of this research include developing a thorough understanding of the effective conductivity in foam insulation samples, as well as understanding how aging and weathering experienced in a cold climate will affect the performance of the materials. In order to obtain these results, initial measurements followed by four phases of testing have taken place. The initial measurement phase will provide insight to the temperature dependent conductivity of the materials when the materials are in pristine condition from the manufacturer. Following these initial measurements, separate samples will undergo further testing through the listed methods:

1) Exposure to a range of humidity levels to determine moisture dependent conductivity.

2) Full submersion in order to assess moisture storage properties of the materials.

3) Elevated temperature exposure in order to simulate accelerated aging.

4) Freeze-thaw cycling in order to determine the effects of weathering on performance.

For these test methods, separate samples have been cut from the same initial sprayed polyurethane sample or polyiso board. This will allow each test to begin with a new sample while still ensuring that all samples of a given material come from the same source, minimizing differences between samples of the same material. For the testing methods involving temperature and humidity set points, data logging humidity and temperature sensors were placed in the chambers in order to ensure a high level of accuracy in environmental set points.

For each material, there are three main test sequences which will be carried out with separate samples. These test sequences will provide a wide scope of measured conductivity values for each material. The following processes describe the sequence of testing and obtained information for each of the material samples. 
Sample 1: Temperature Dependent Conductivity and Elevated Temperature Aging

1) The temperature dependent conductivity of this sample will be measured using a heat flow meter.

2) The sample will be exposed to an elevated temperature in order to accelerate aging processes.

3) The temperature dependent conductivity will be measured throughout the accelerated aging process.

The results from sample 1 will provide insight into the long term performance of the insulating material across a range of temperatures. Through the measurements taken at set intervals, the rate of aging effects will be observed.

Sample 2: Effective Conductivity as a Function of Temperature and Moisture

1) The sample will be dried and the temperature dependent conductivity will be measured.

2) The sample will be exposed to increasing humidity levels. The mass and temperature dependent conductivity will be measured after the sample has acclimated to each humidity set point.

3) The sample will be fully submerged. The mass will be measured after one week of exposure.

The results from sample 2 will provide a set of temperature dependent conductivity measurements, over a range of humidity levels. These measurements will be used in developing 3-D surface models used to calculate the effective conductivity of the sample. 
Sample 3: Freeze-Thaw Cycling to Assess Effects of Environmental Weathering Over Time

1) The sample will be exposed to 150 freeze-thaw cycles. The temperature dependent conductivity will be measured after 75 and 150 cycles.

2) The sample will be exposed to increasing humidity levels. The mass and and temperature dependent conductivity will be measured after the sample has acclimated to each humidity set point.

3) The sample will be fully submerged. The mass will be measured after one week of exposure.

The results from sample 3 will indicate how the material strains caused by freeze-thaw cycling affect the performance of the sample. It will also provide results that can be compared with sample 2 in order to determine the effects of freeze-thaw cycling on the moisture storage capabilities of the sample. The materials investigated are discussed in further detail in the following sections. 


\subsection{Material Samples}

The material samples used in the testing include various types of polyurethane (referred to as $\mathrm{PU}$ ) and polyisocyanurate (referred to as PI). The assortment of materials was chosen to span over the range of commonly used insulations types. In total, six materials were fully assessed in this study. These six materials include two closed-cell polyurethanes, two open-cell polyurethanes, and two closed-cell polyisocyanurates. The two closed-cell polyurethane materials (PU-A and PU-B) from separate manufacturers are similar in their thermal performance and density, and reflect standard medium-high density polyurethane. The two open cell polyurethane materials are very different from each other. PU-C reflects standard low density open-cell foam insulation, while the PU-D material is novel open cell foam that is advertised to act as a vapour permeable exterior insulated sheathing membrane which has a relatively high density for open cell foams. While open cell foams theoretically avoid some of the aging effects due to the open cells being filled with air rather than blowing agents, it will still be important to investigate how the aging and weathering affects these samples. The polyisocyanurate sample PI-A is a high performance aluminum faced material designed to perform in assemblies with thickness limitations, and for this reason it is manufactured in sheets $25 \mathrm{~mm}$ thick. Material PI-B reflects a standard polyisocyanurate board, with a cardboard facing, available in $50 \mathrm{~mm}$ sheets. Table 3.1 reports the advertised conductivity and density of the selected materials which are being investigated.

Table 3.1: Investigated Material Properties

\begin{tabular}{ccc}
\hline Material & $\begin{array}{c}\text { Conductivity } \\
{[\mathrm{W} / \mathrm{m} \mathrm{K}]}\end{array}$ & $\begin{array}{c}\text { Density } \\
{\left[\mathrm{kg} / \mathrm{m}^{3}\right]}\end{array}$ \\
\hline Polyurethane-A (Closed Cell) $35 \mathrm{~mm}$ & 0.0233 & 28.9 \\
Polyurethane-B (Closed Cell) $50 \mathrm{~mm}$ & 0.025 & 35.0 \\
Polyurethane-C (Open Cell) $50 \mathrm{~mm}$ & 0.0378 & 6.83 \\
Polyurethane-D (Open Cell) 50mm & 0.0333 & 17.2 \\
Polyisocyanurate-A (Closed Cell) $25 \mathrm{~mm}$ & 0.0173 & 32.0 \\
Polyisocyanurate-B (Closed Cell) $50 \mathrm{~mm}$ & 0.0248 & 32.0 \\
\hline
\end{tabular}


For the tests, samples of the same materials were cut to equal thickness. While the objective was for all samples to be cut to a thickness of $50 \mathrm{~mm}$, due to irregularities with some of the manufacturer provided samples, some material samples were cut to be slightly thinner in order to obtain uniform samples.

Additionally, for PI-A, this material was only available in $25 \mathrm{~mm}$ thick sheets. In Fig. 3.1, images of the investigated materials are shown.

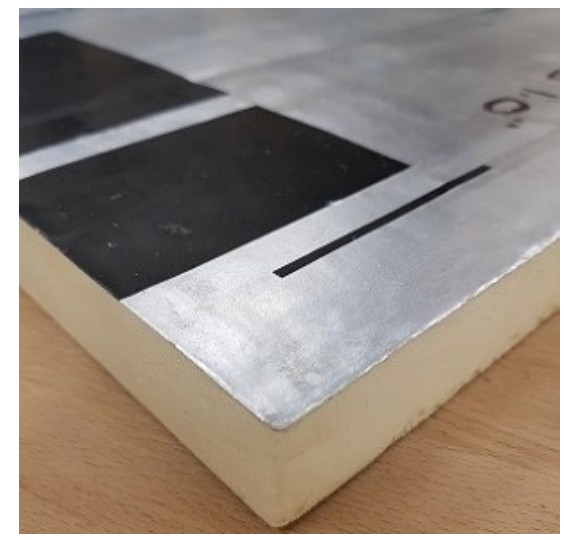

PI-A

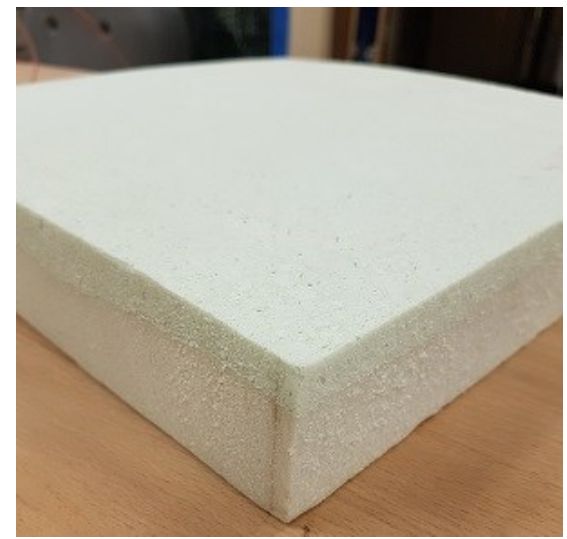

PU-B

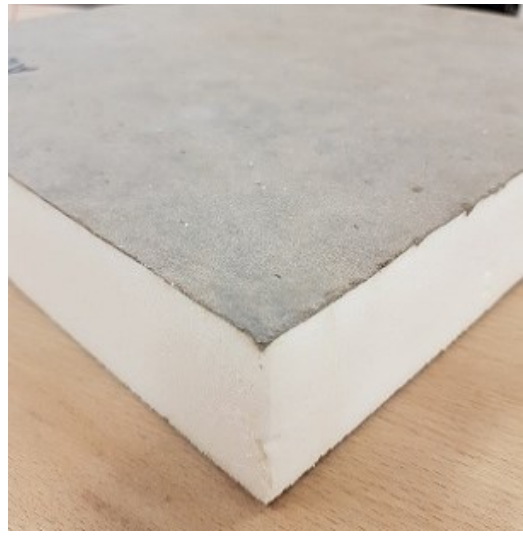

PI-B

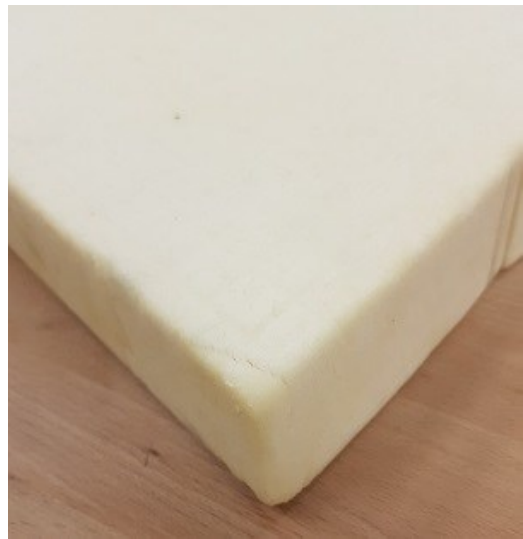

PU-C

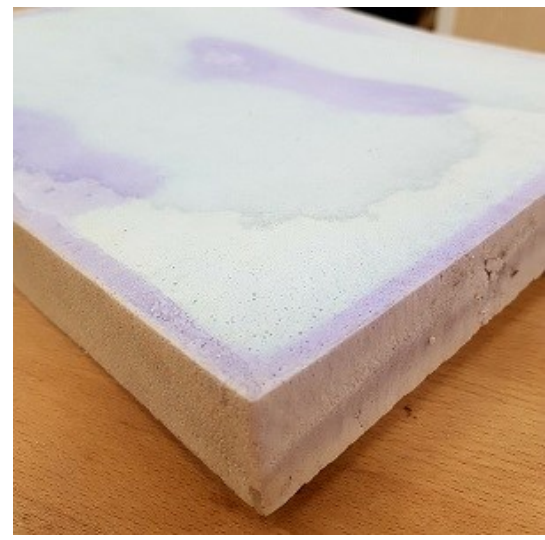

PU-A

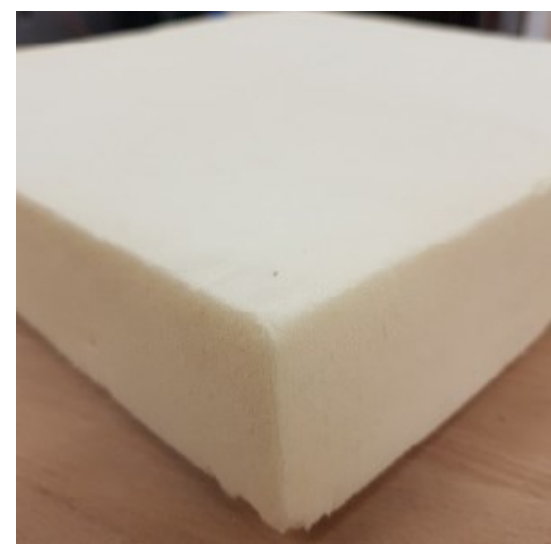

PU-D

Figure 3.1: Investigated Material Samples 


\subsection{Temperature Dependent Conductivity}

For each material sample, the initial step involved testing each sample in a heat flow meter, which will allow the conductivity of a given material to be tested at a set mean temperature as well as a set temperature delta. For all conductivity measurements taken for this portion of testing as well as all other test methods, the 436 Lambda model heat flow meter equipment, manufactured by Netzch Germany was used. This equipment functions through the use of two heat plates that provide a temperature delta, between which a material sample is placed. The sample conductivity is measured over a range of mean temperature set points. According to the CAN/ULC-S770-15 " Standard Test Method for Determination of Long-Term Thermal Resistance of Closed-Cell Thermal Insulating Foams, materials should be tested in accordance with ASTM C518 at a mean temperature of $24^{\circ} \mathrm{C}(+/-$ 2) and a delta of $22^{\circ} \mathrm{C}(+/-2)$. In order to gain a full understanding of the material performance in a Canadian climate the samples will also be tested at a range of mean temperatures from $-10^{\circ} \mathrm{C}$ to $30^{\circ}$ $\mathrm{C}$ with a delta of $20^{\circ} \mathrm{C}$, in order to determine the temperature dependent conductivity. Due to limitations with the cooling power of the testing equipment, the tests at a mean temperature below $0^{\circ}$ $\mathrm{C}$ ran with a temperature delta reduced to $10^{\circ} \mathrm{C}$ down from $20^{\circ} \mathrm{C}$. For each of these tests, the heat flow meter performed within an accuracy range of $\pm 1.0 \%$ in conductivity readings.

These measurements will indicate how each of the samples perform over a range of temperatures typically found in a Canadian climate. Through this portion of testing it will be determined whether a certain sample will behave with a linear temperature dependent conductivity or if the material experiences irregular effective conductivity trends as some research suggests.

The measurements will be used as the benchmark for continued investigation. As the materials undergo simulated environmental weathering, it will be understood how the performance of these materials will change over their life-cycle and under varying conditions. 


\subsection{Moisture Dependent Conductivity Testing}

Prior to beginning moisture testing, a customized humidity chamber has been built in order to create and environment with controlled temperature and humidity. This chamber is used for exposing material samples to a constant set environment. The chamber consists of a silicone sealed acrylic panel box, covered with a 50mm layer of rigid XPS insulation board in order to hold a set temperature and humidity level. The chamber houses the following equipment that control the humidity level and temperature within the chamber:

1) Humidifier: Crane Ultrasonic room humidifier, filled with distilled water

2) Dehumidifier: Homasy Portable 22W air dryer

3) Heat Source: Sylvania 175W Infrared Heat Lamp

The humidifier and dehumidifier are controlled by an Inkbird programmable digital humidity controller which activates the humidifier and dehumidifier separately as the humidity level in the chamber falls below or increases above the set point. A second Inkbird programmable outlet/thermostat controls the heat source, keeping a steady temperature within the chamber. Using this equipment, a relatively steady humidity and temperature level can be held within the chamber separately in order to achieve the required humidity level. The limitation of the equipment causes the relative humidity to fluctuate over a range of $\sim 5 \% \mathrm{RH}$ and the temperature to fluctuate over a range of $\sim 4^{\circ} \mathrm{C}$. The exterior and interior of the custom chamber can be seen in Fig. 3.2. 


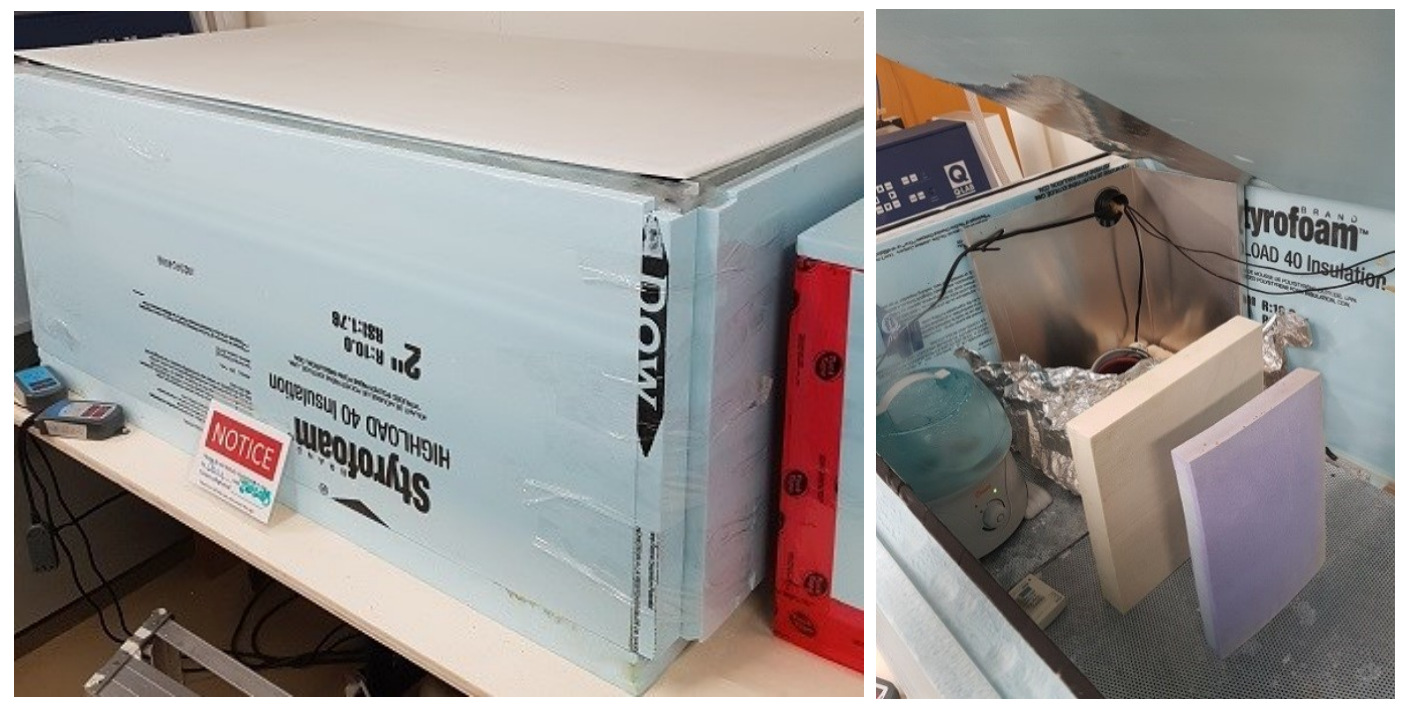

Figure 3.2: Custom Built Environmental Chamber

For the purpose of determining the moisture dependent conductivity of the materials, this chamber was used to expose the materials to environments of $40 \%, 80 \%, 90 \%$, and $95 \% \mathrm{RH}$ and a constant temperature of $25^{\circ} \mathrm{C}$ for at least seven days at each set point. Additionally, prior to these humidity exposure tests, the samples were dried in a microprocessor controlled mechanical convection oven $(\sim 10-15 \% \mathrm{RH})$ at $40^{\circ} \mathrm{C}$. These samples provided conductivity and mass measurements for the materials in an assumed dried state. After the materials have been acclimated to each set point, they were weighed in order to determine moisture storage capacity over a range of humidities, then were wrapped with a non-insulating, vapour impermeable wrapping in order to preserve the state of the material throughout the measurement duration in the heat flow meter.

As the water storage function of materials generally shows that foam materials, especially closed-cell materials, absorb more water content only at very high moisture levels. Focus was placed on set points above $80 \% \mathrm{RH}$ in order to observe the critical changes in the effective thermal conductivity and moisture content at high moisture levels, compared to the set points below $80 \%$ which are expected to see minimal differences in the material performance. 


\subsection{Submersion Testing}

Closely related to the previous section, through submersion testing, the objective is to determine the maximum moisture storage capacity of each material. For this test method, samples are submerged in filtered water for an extended period of time. Plastic tanks were built with a mechanism to hold the buoyant samples below the waterline, where the materials are also separated by plastic grates to allow maximum surface area exposure to water. The submersion tanks can be seen in Fig. 3.3. For these materials, submerged weights will be obtained after 1 week of submersion and again after 4 weeks.
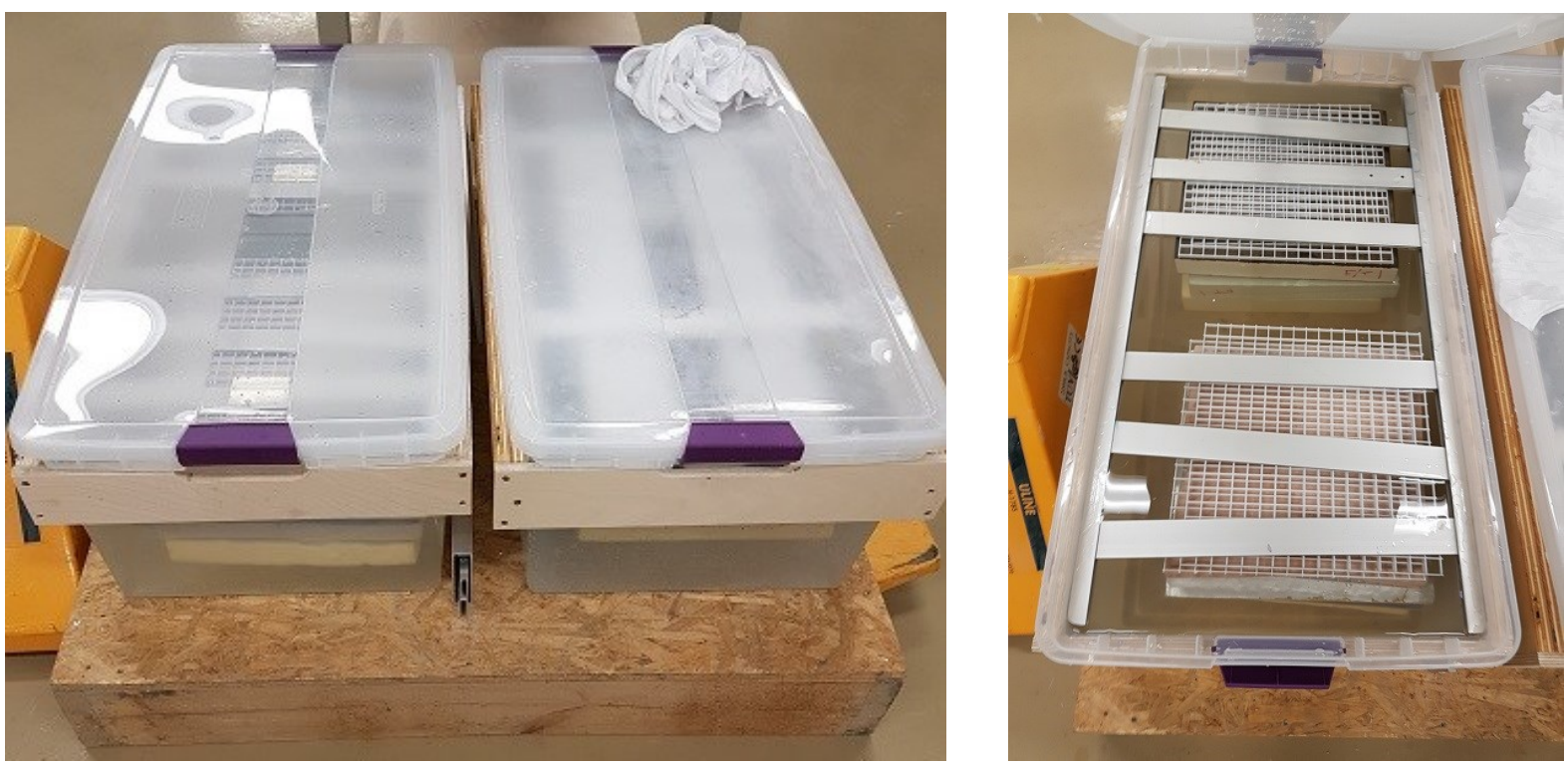

Figure 3.3: Custom Built Submersion Tanks 


\subsection{Freeze-Thaw Cycling}

Research has suggested that undergoing many freeze-thaw cycles may increase the water storage capacity of foam materials due to degradation of the foam polymers (Garber-Slaght, 2012). Consequently, the effect of freeze-thaw cycling was investigated on the materials regarding both their effective conductivity and moisture storage capacity. As the materials will experience many freezethaw cycles in a cold climate, this method provides insight as to how the materials will be affected over time in a realistic setting.

The test chamber for this method is a CF-2060 freezer (Salton Canada) which was modified to contain a 100W fan heater (STEGO England) (Fig.3.4). The components are controlled by cycled between $40^{\circ} \mathrm{C}$ and $-30^{\circ} \mathrm{C}$ on a 24-hour loop, each temperature set point was held for approximately 10 hours, with approximately 2 hours of transition between set points. Details of set point selection correspond with similar testing done by Berardi \& Nosrati (2017). The materials were exposed to approximately 150 freeze-thaw cycles, and their thermal conductivities were measured after 75 cycles, and again after 150 cycles. Following the freeze-thaw cycling tests, samples were also exposed to high moisture levels (80, 90, 95\% RH and submerged) in accordance to the methodology outlined in sections 3.3-3.4 in order to determine the extent of increased moisture storage capacity caused by the freeze-thaw cycling in addition to any effects on the effective conductivity due to the cycling alone.
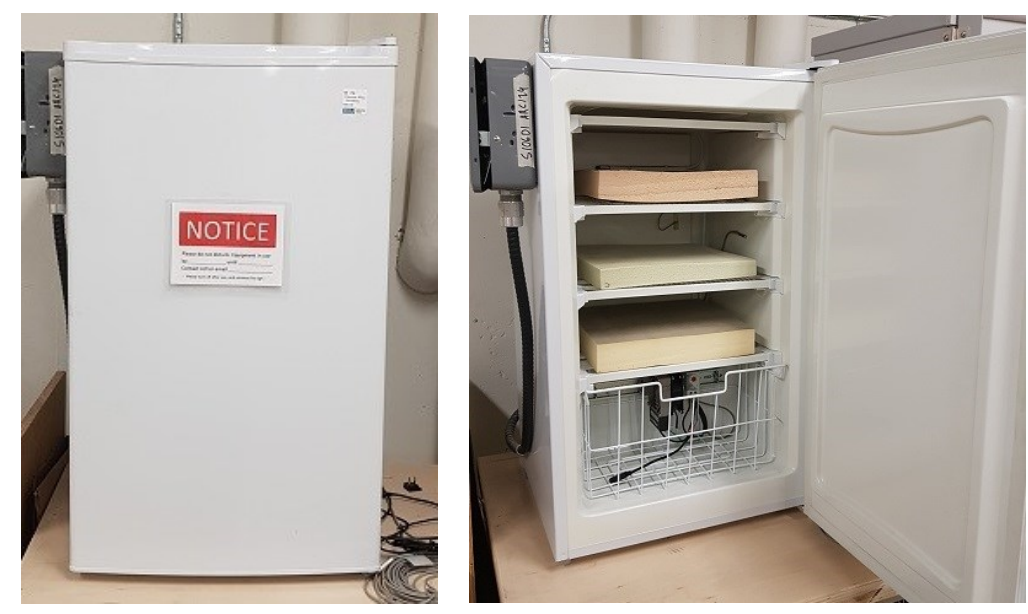

Figure 3.4: Freeze-Thaw Chamber 


\subsection{Elevated Temperature Accelerated Aging}

The most important aspect of the simulated aging method is not just to determine a single conductivity value such as the LTTR, but to understand how the effective conductivity over a range of temperatures is affected over the aging process. Through accelerated aging by means of elevated temperature, the material samples can move along an aging curve within much shorter periods. A microprocessor controlled mechanical convection (Fig 3.5) oven set at $60^{\circ} \mathrm{C}$ was used in this study and samples were aged for up to 4 months. Material conductivities were measured monthly in order to gain a better understanding of the performance over the aging process. Based on research regarding methodologies for accelerated aging, an elevated temperature of $60^{\circ} \mathrm{C}$ was selected in order to limit polymer damage from excessively high temperatures that may cause damage beyond what is experienced in a realistic setting, while still providing an environment to promote accelerated aging (Hunter et al, 2000).

Through this method, an understanding of the aging process effect on materials will be developed. While the results of the elevated temperature aging tests will provide important insights into the performance of materials, it is also important to contextualize the results through determining an equivalency between the accelerated aging duration and real time aging under lab conditions. Using the Arrhenius Equation (Eq.2), the time spent aging at $60^{\circ} \mathrm{C}$ can be equated to real time under standard lab conditions (Berardi \& Nosrati, 2017).

$$
A F_{T}=e^{-\frac{E_{A}}{K} \cdot\left(\frac{1}{T_{A}}-\frac{1}{T_{U}}\right)} \quad \text { Eq. } 2 \text {-Arrhenius Equation }
$$

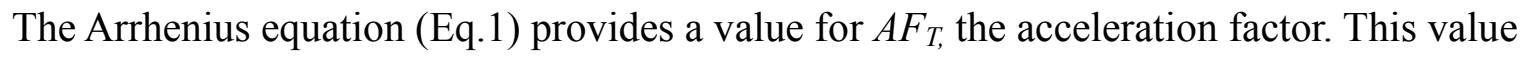
describes the rate at which a material ages at an elevated temperature $\left(T_{A}\right)$ relative to the use temperature $\left(T_{U}\right)$. The use temperature being the temperature you expect the material to be exposed to during use, or a temperature to be compared to the elevated temperature. The equation also uses $(K)$, 
the Boltzmann constant $\left(8.617 \times 10^{-5} \mathrm{eV} / \mathrm{K}\right)$. The key to the Arrhenius equation is the activation energy value for a material $\left(E_{A}\right)$. This value is the minimum energy required for chemical reactions to occur in a given material. After aging results are obtained, this equation will aid in the analysis of the effective conductivity in foam insulation materials throughout and after the process.
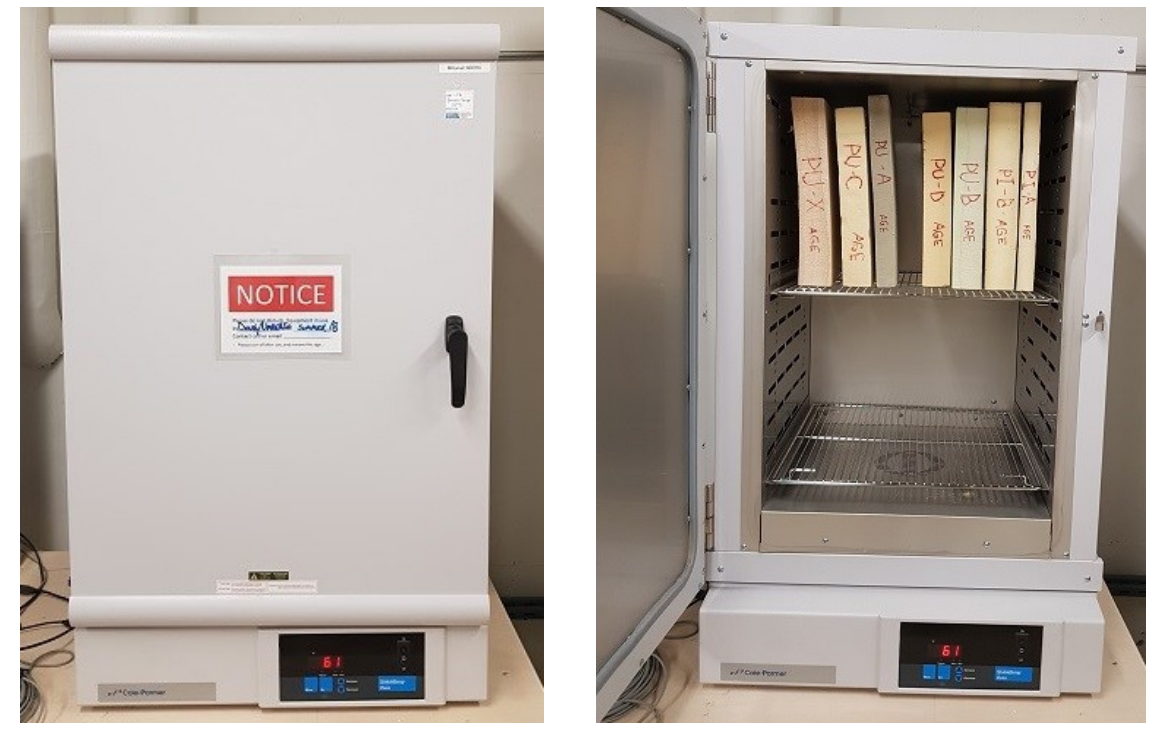

Figure 3.5: Elevated Temperature Aging Oven 


\subsection{Measured Results}

In this section, the measured results for the selected set of materials after undergoing the test methodologies previously described are reported and discussed. Results include initial measured temperature dependent conductivity curves, as well as additional temperature dependent conductivity curves over a range of moisture levels, 3-D representations of material effective conductivity, the effect of elevated temperature on temperature dependent conductivity over time, and the effect of freeze-thaw cycling on the effective conductivity and moisture storage properties of materials. 


\subsection{Temperature Dependent Conductivity}

Through the initial measurements using the heat flow meter, the material samples were tested in order to determine the benchmark temperature dependent conductivity of those materials. The results were also plotted against the assumed constant value of the manufacturers advertised conductivity.

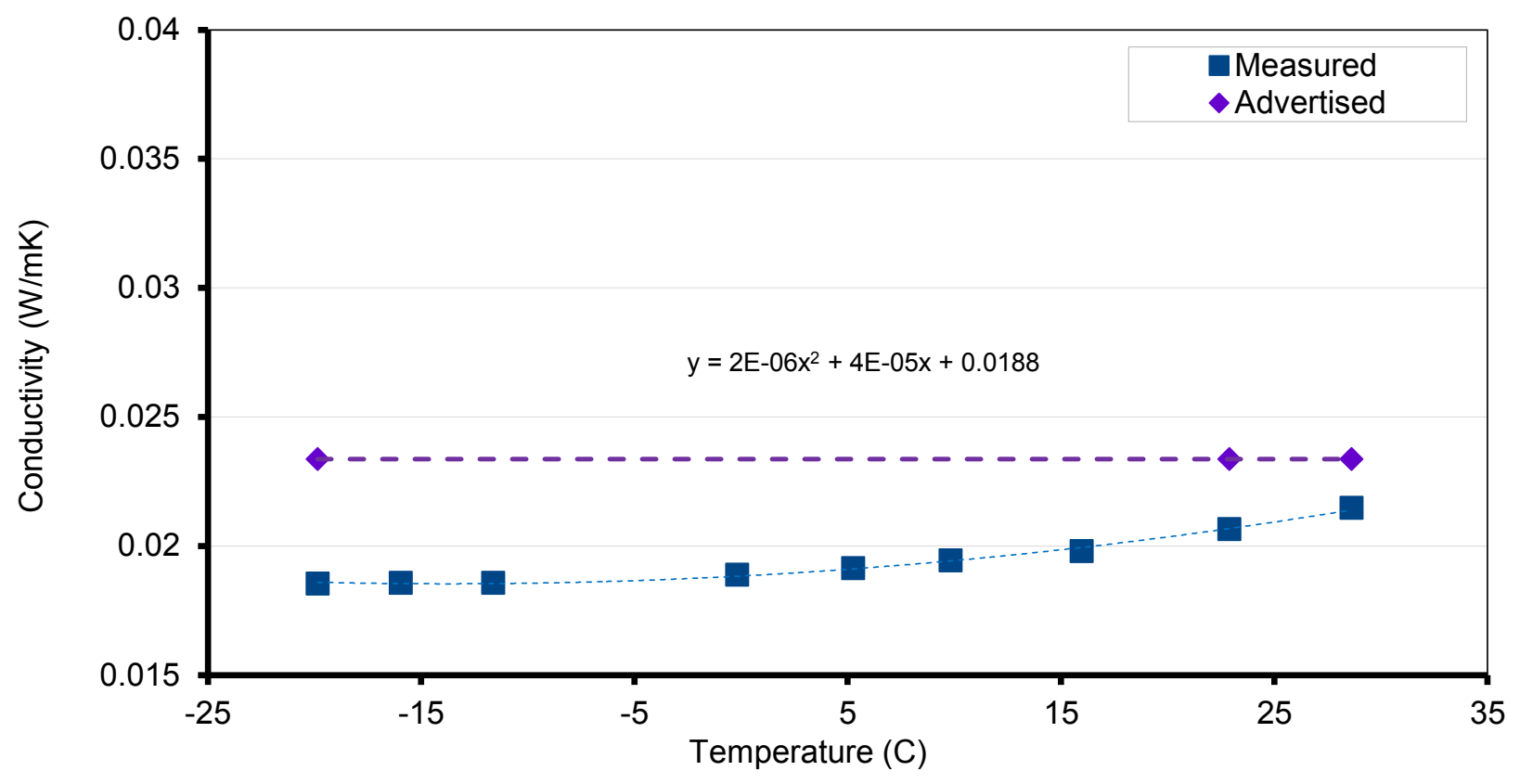

Figure 4: Initial Temperature Dependent Conductivity of Polyurethane A

In the first material test for Polyurethane A (Fig. 4.1), the conductivity is increasing at a relatively linear rate between $0 \mathrm{C}$ and $30 \mathrm{C}$. This reveals that contrary to what some research suggests (Tseng, 1997), this polyurethane sample does not appear to have a major increase in conductivity immediately below $0^{\circ} \mathrm{C}$, though the value towards $-20^{\circ} \mathrm{C}$ does appear to plateau, no longer scaling linearly with temperature. This may indicate that at even colder temperatures there could be irregular effects to assess, though for mean temperatures below -20 , lab testing is not feasible, due to constraints on the lab equipment used. At higher temperatures, the conductivity seems to increase at a relatively linear rate, which is consistent with general research regarding temperature-dependent conductivity of most insulation products (Grin et al, 2014). 
The results of Polyurethane B, (Fig. 4.2) reveal a similar trend to the previous polyurethane sample, with conductivity increasing relatively linearly towards $25^{\circ} \mathrm{C}$. Though in this sample, the trend remains relatively linear into colder temperatures.

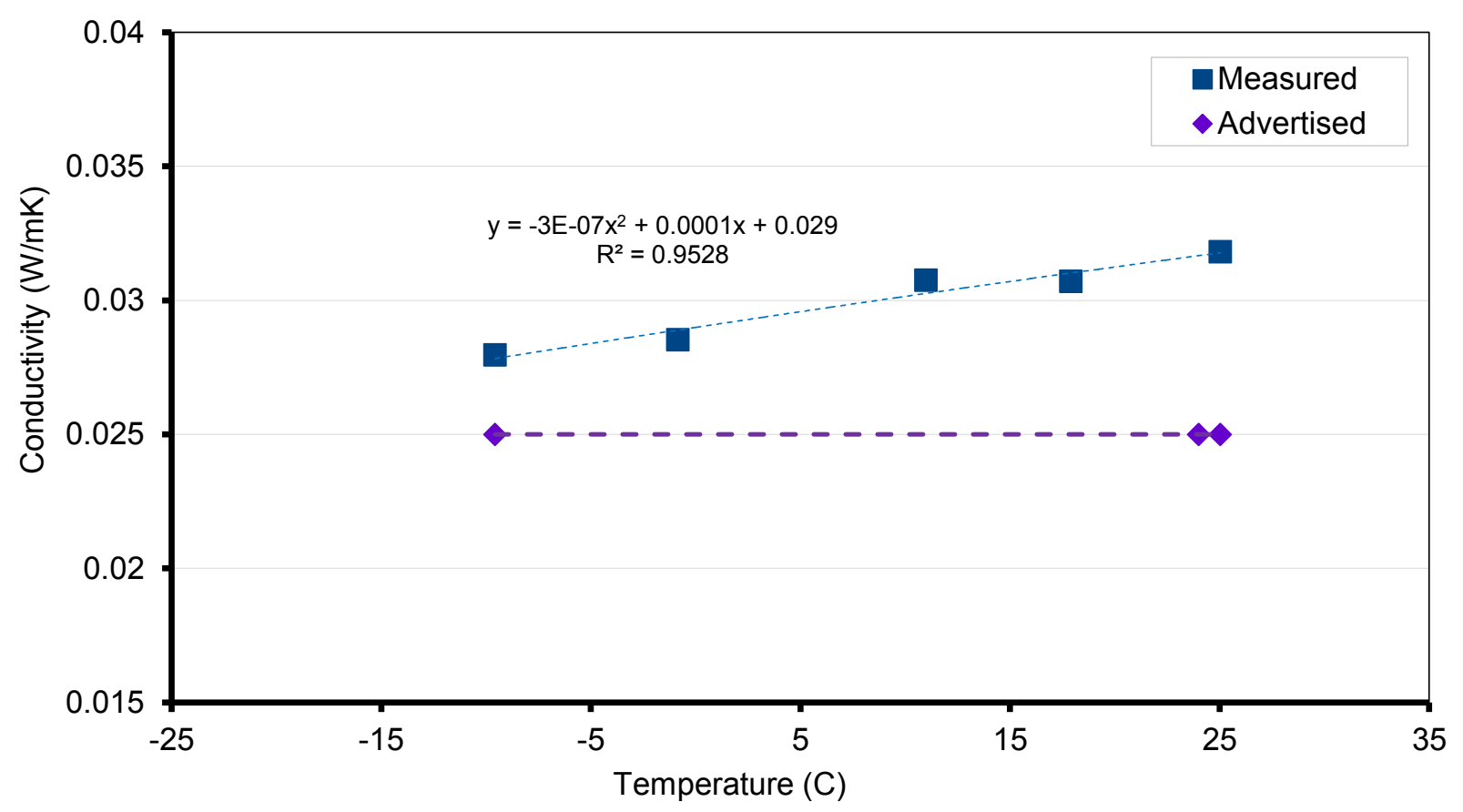

Figure 4.2: Initial Temperature Dependent Conductivity of Polyurethane B

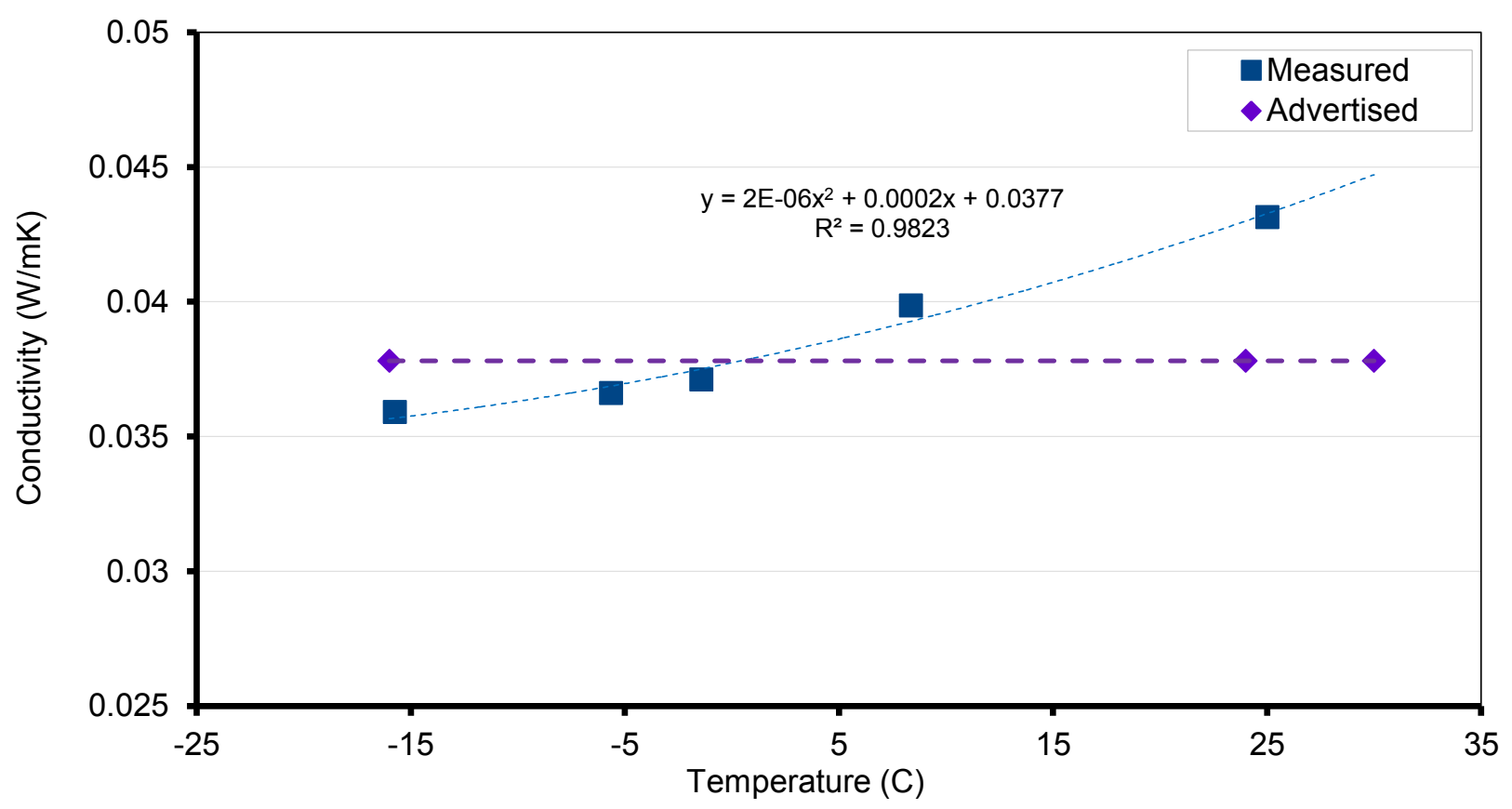

Figure 4.3: Initial Temperature Dependent Conductivity of Polyurethane C 


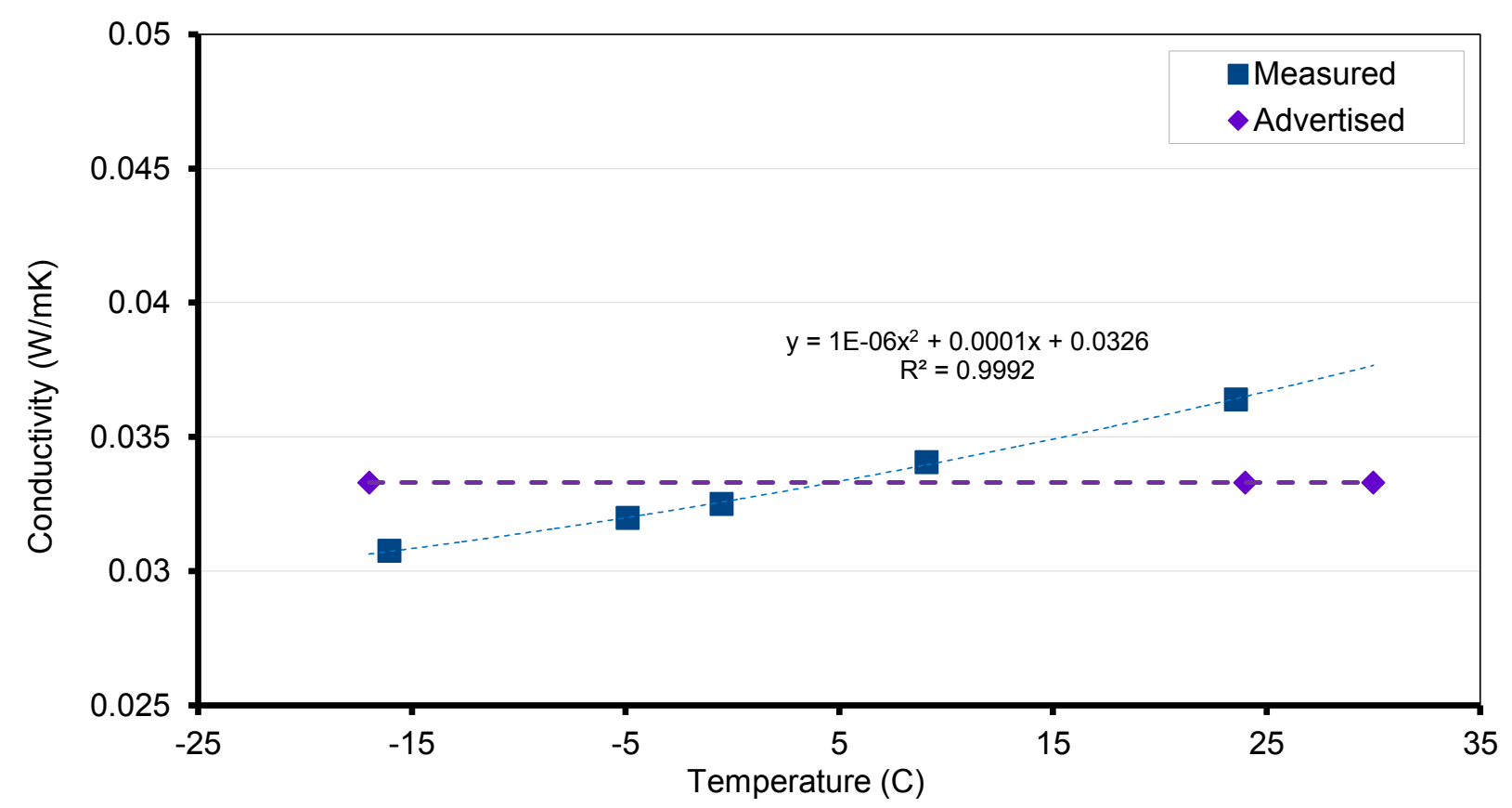

Figure 4.4: Initial Temperature Dependent Conductivity of Polyurethane D

In Polyurethane samples C and D (Fig. 4.3-4.4), relatively linear correlations between conductivity and temperature are seen once again. These four initial results for polyurethane samples indicate that for these materials, the temperature dependent conductivity is relatively consistent with the general assumptions of a linear correlation. However, in the polyisocyanurate materials, the trendbreaking effect of increasing conductivity at low temperatures as indicated in previous research (Grin et al, 2014) was seen. The test result of Polyisocyanurate sample A (Fig.4.5) indicates there is an effect of increasing conductivity at temperatures below $0^{\circ} \mathrm{C}$. From approximately $5^{\circ} \mathrm{C}$ and lower, the conductivity appears to increase until $-15^{\circ} \mathrm{C}$, where it begins to plateau. In this sample, there is $\sim 18 \%$ increase in conductivity from the lowest conductivity measured at $5^{\circ} \mathrm{C}$, compared to the highest at $20^{\circ} \mathrm{C}$. Contrary to the plateau in conductivity below $-15^{\circ} \mathrm{C}$ in PU-A, polyisocyanurate sample B (Fig. 4.6) experienced an increase in conductivity at a much higher rate. Between the lowest and highest conductivity measured for sample $\mathrm{B}$, there is $33 \%$ reduction in performance at $-20^{\circ} \mathrm{C}$ compared to the lowest measured value at $10^{\circ} \mathrm{C}$, 


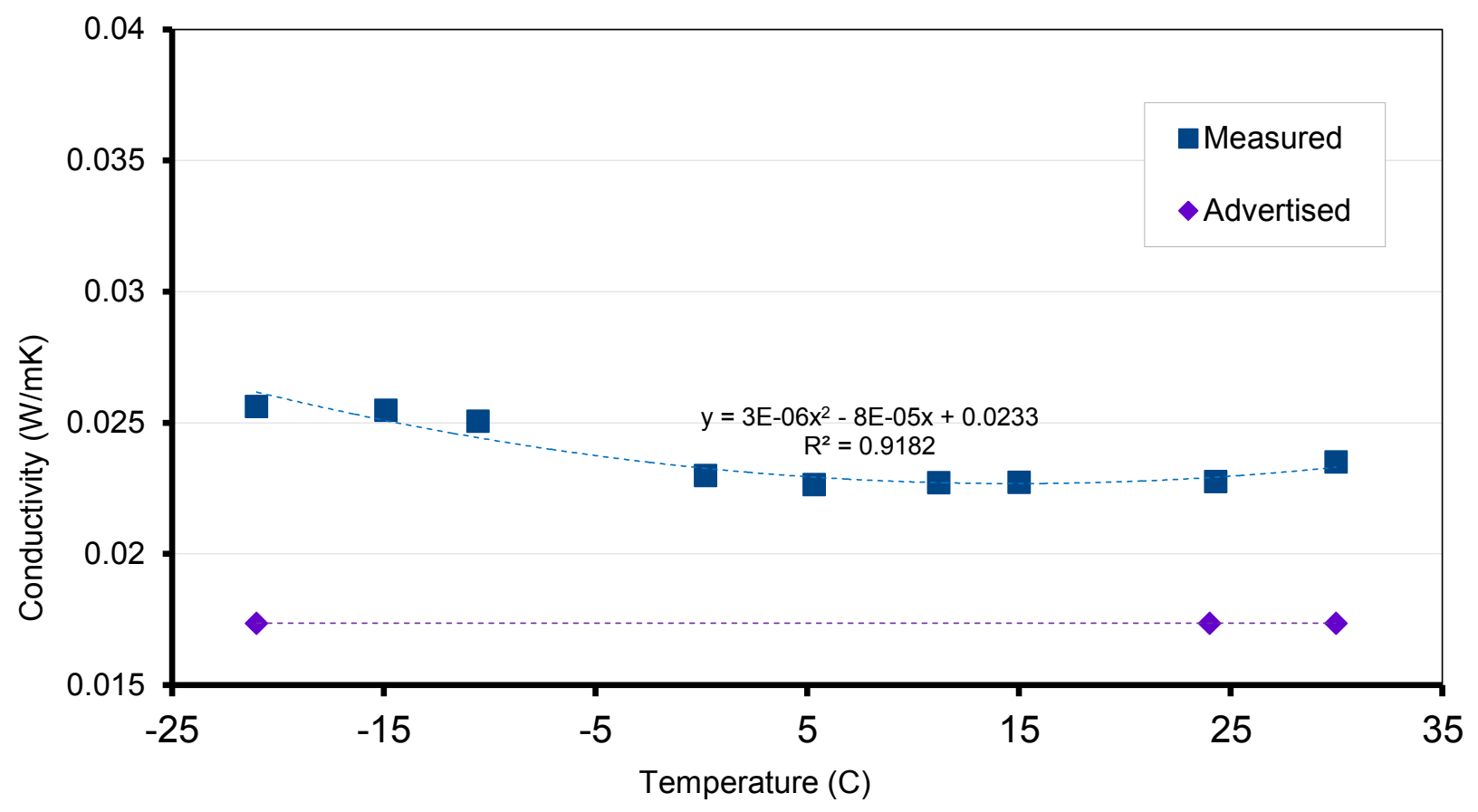

Figure 4.5: Initial Temperature Dependent Conductivity of Polyisocyanurate A

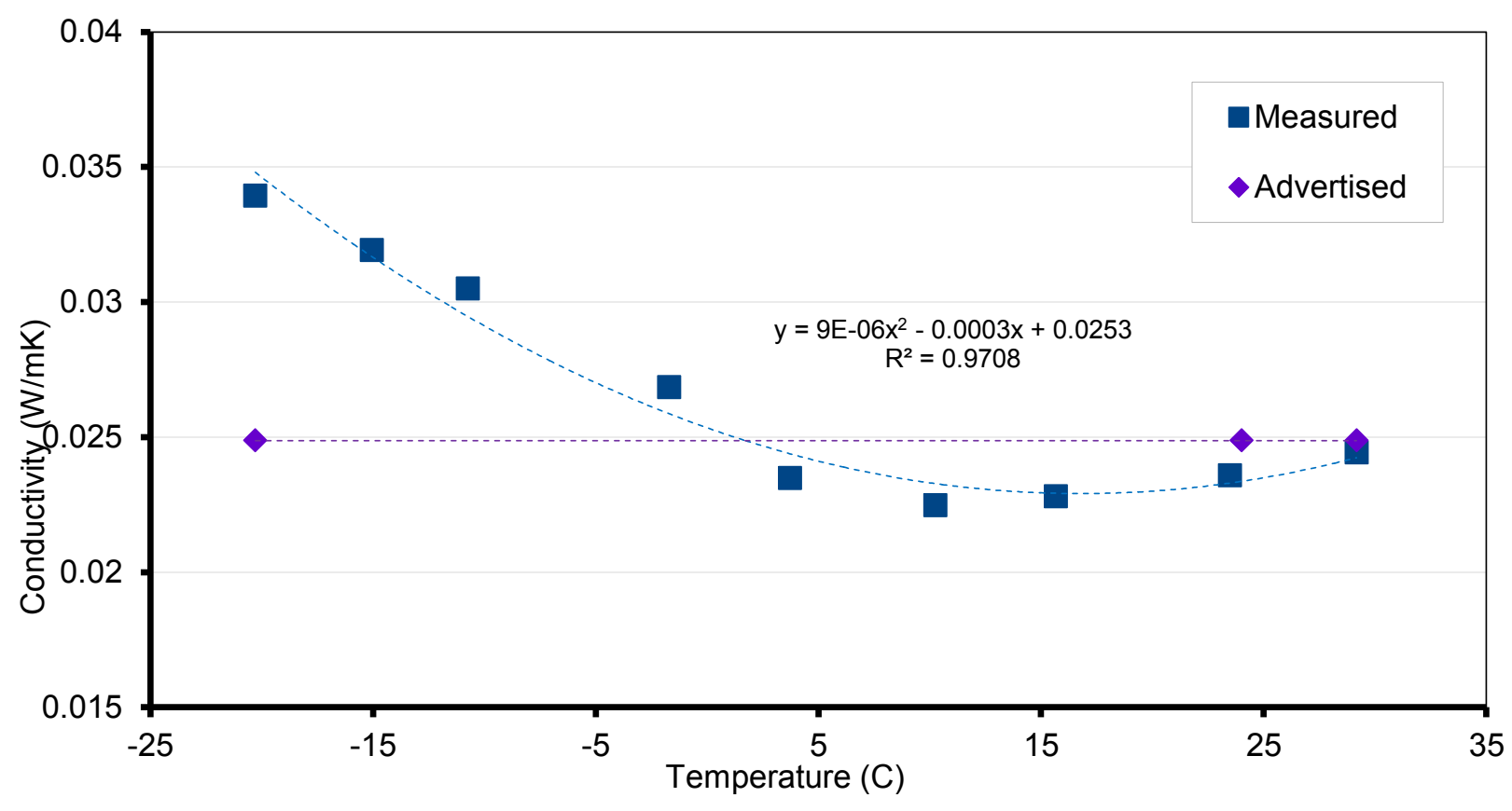

Figure 4.6: Initial Temperature Dependent Conductivity of Polyisocyanurate B 
An important secondary observation that was made looking at all five of the material test results is the inconsistencies between the advertised value and the measured value of materials. Looking at the initial results, the measurements suggest that in a scenario where an assembly is designed with a specific U-value, the wall may perform much differently than the advertised value suggests.

From Fig. 4.7, the range of conductivities measured in each material can be easily compared with each other. It can be clearly seen that while the polyurethane samples indicate an expected linear increase in conductivity with increasing temperature. In the polyisocyanurate samples it is clear that something in the material causes an unexpected increase in conductivity at low temperatures. The result of PU-A also suggests that as the temperature is further reduced below $-20^{\circ} \mathrm{C}$, this effect may begin to appear, indicated by the plateau of measured values in the $-10^{\circ} \mathrm{C}$ to $-20^{\circ} \mathrm{C}$ range, mimicking what is seen in the PI-A and PI-B results in the $5^{\circ} \mathrm{C}$ to $15^{\circ} \mathrm{C}$ range.

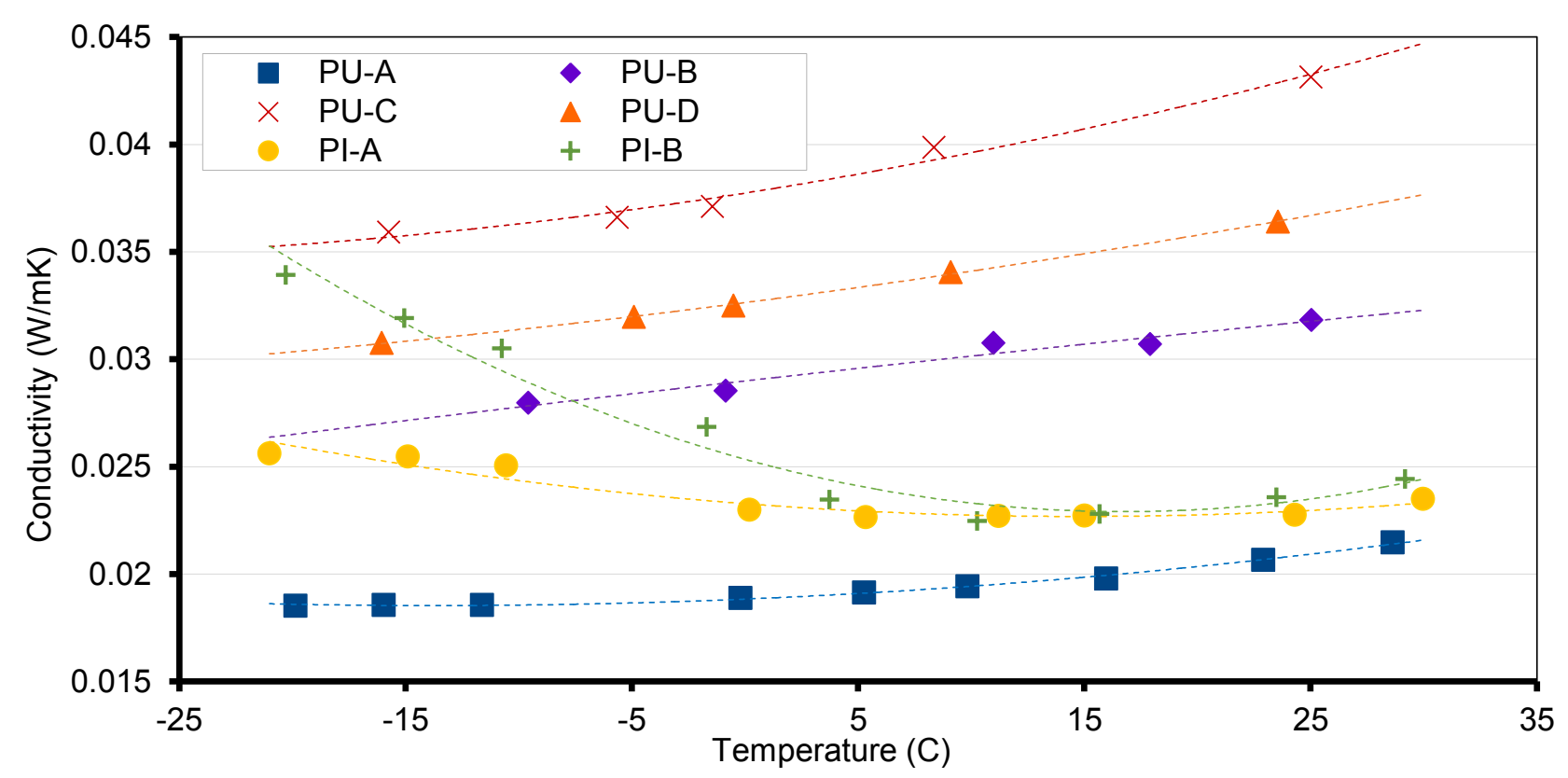

Fig 4.7: Measured Temperature Dependent Conductivity for All materials

The following sections will show the effects of the test methods on the effective conductivity for these materials. Important effects to be aware of include to what extent the test methods change the value of conductivity, as well as whether the test methods change the shape of the effective 
conductivity curves, indicating whether the material will perform better or worse under certain conditions after aging effects.

The initial measurements exploring temperature dependent conductivity revealed that the effects of condensing blowing agents causing an increase in conductivity at low temperatures are mostly seen in the polyisocyanurate materials, with only one closed cell polyurethane samples experiencing a similar effect to a very minimal degree. For this reason, it can be assumed that the cause of the unusual effect seen in these materials is due to blowing agents in the materials. The exact nature of the blowing agents used have not been disclosed by the manufacturers, but through research by Lett et al. in 2016, we can likely assume that the polyisocyanurate samples that were tested use some form of the problematic Pentane blowing agent, which were shown to condense at low temperatures causing the increase in conductivity seen in the results below $0^{\circ} \mathrm{C}$. Based on the results of the closed-cell polyurethanes that were tested, it seems that within the expected range of temperatures experienced in a Canadian climate, that the fresh samples did not experience the negative effects of increasing conductivity below $0{ }^{\circ} \mathrm{C}$. According to Tseng (1997), the issue of increasing conductivity below $0{ }^{\circ} \mathrm{C}$ was due to the condensation of interior gasses in the closed cells of the foam. The lack of this effect being observed could be explained by differences in the blowing agents used in polyurethane foam insulations compared to when Tseng was conducting his testing prior to regulations phasing out common blowing agents from the time. While the initial measured results imply that the current HFC gasses used in Polyurethane foams do not encounter the condensation effect to the extent that polyisocyanurate does, however for PU-A, there was a slight increase in conductivity towards $-15^{\circ} \mathrm{C}$ after lab testing as indicated in the elevated temperature and moisture testing results. This suggests that the HFC gasses used in polyurethanes may also experience condensation at low temperatures, though the effects seem to begin at lower temperatures than seen in polyisocyanurate and varying between materials. 


\subsection{Elevated Temperature Aging}

Through aging the foam material samples in an oven at $60 \mathrm{C}$ for up to 4 months per sample, minimal changes in thermal performance were seen in three of the four polyurethane samples, while relatively significant changes were seen in one polyurethane sample and both polyisocyanurate samples. In Figs. 4.8-4.11, the measured results for material PU-A, PU-B, PU-C and PU-D respectively are shown.

PU-A (Fig. 4.8) reflects the idea that closed cell polyurethane foam materials age faster early in the process and eventually reach a plateau. The change in the first month of aging is greater than the rate of change later in the process. It is also interesting to note that as this material ages, an increase in conductivity at low temperatures appears. This potential for this effect to occur was identified based on the initial measurement, and after accelerated aging, it appears that while the extent of increasing conductivity at low temperatures is not as high as in the polyisocyanurate materials within this temperature range, it does still occur. Overall, after aging, PU-A remains a relatively consistent performing material, with the conductivity still changing very minimally over a range of temperatures, though the conductivity range as a whole increased from the original measurement.

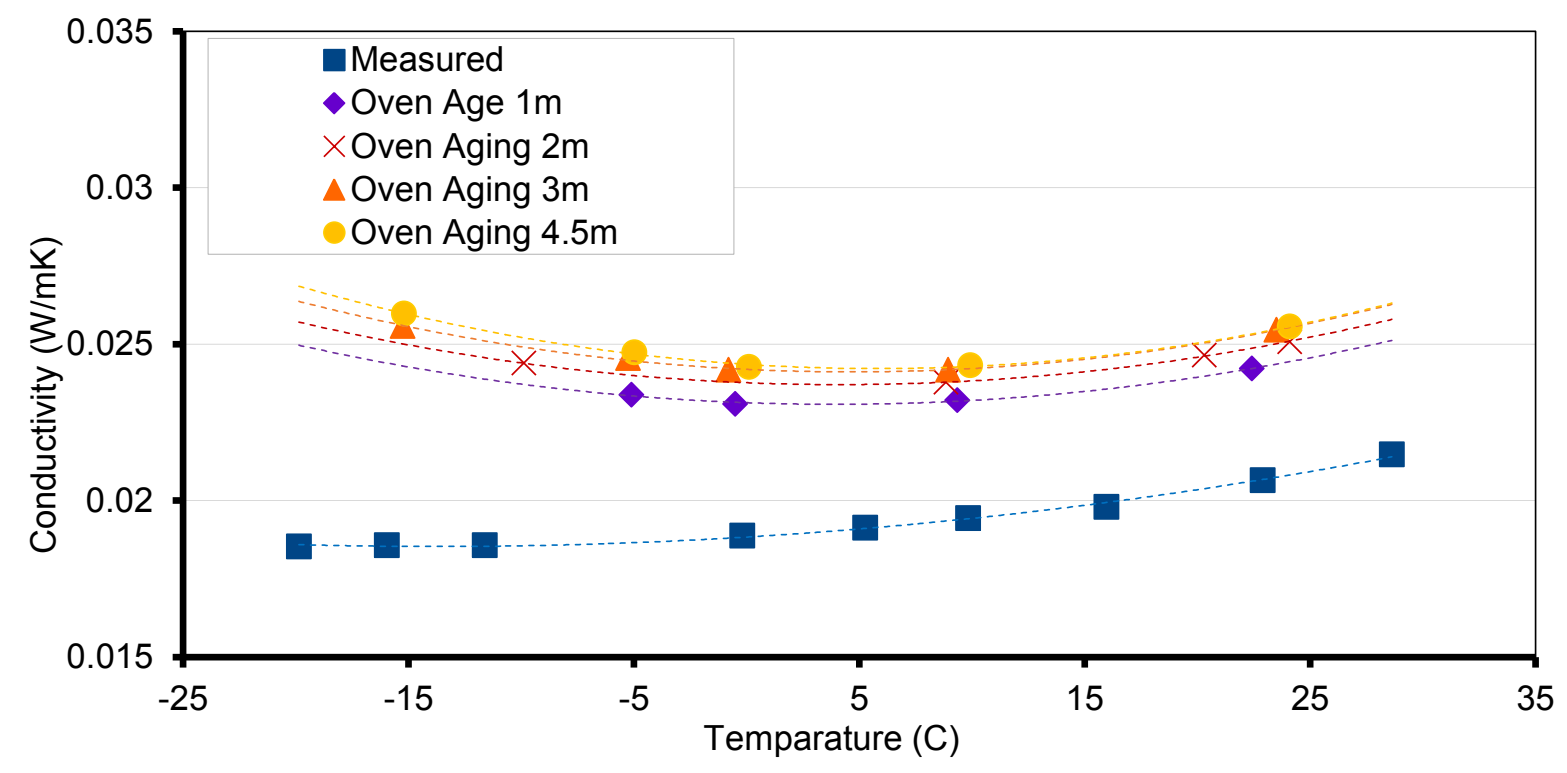

Figure 4.8: Effective Conductivity of Aged Closed Cell Polyurethane (PU-A) 


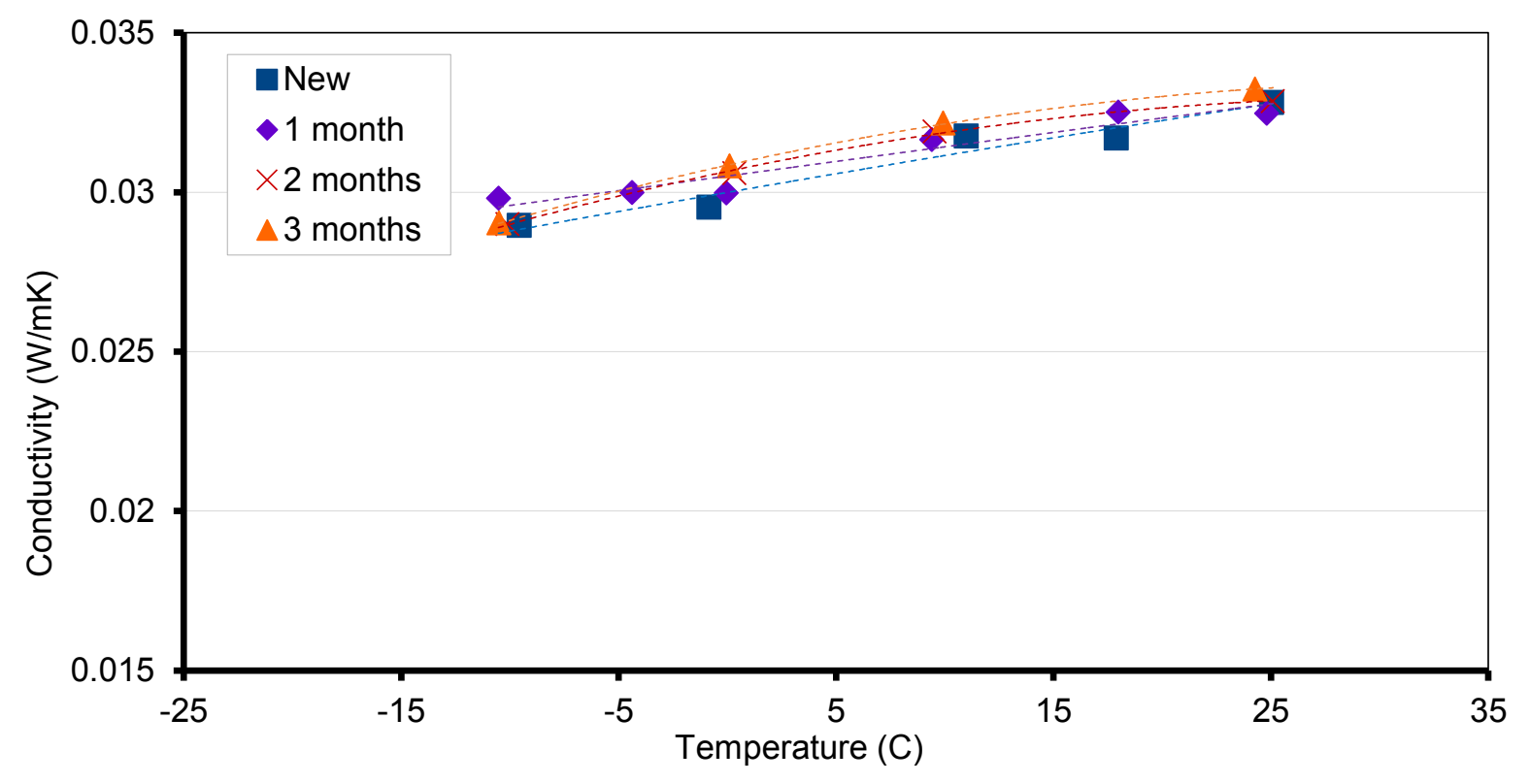

Figure 4.9: Effective Conductivity of Aged Closed Cell Polyurethane (PU-B)

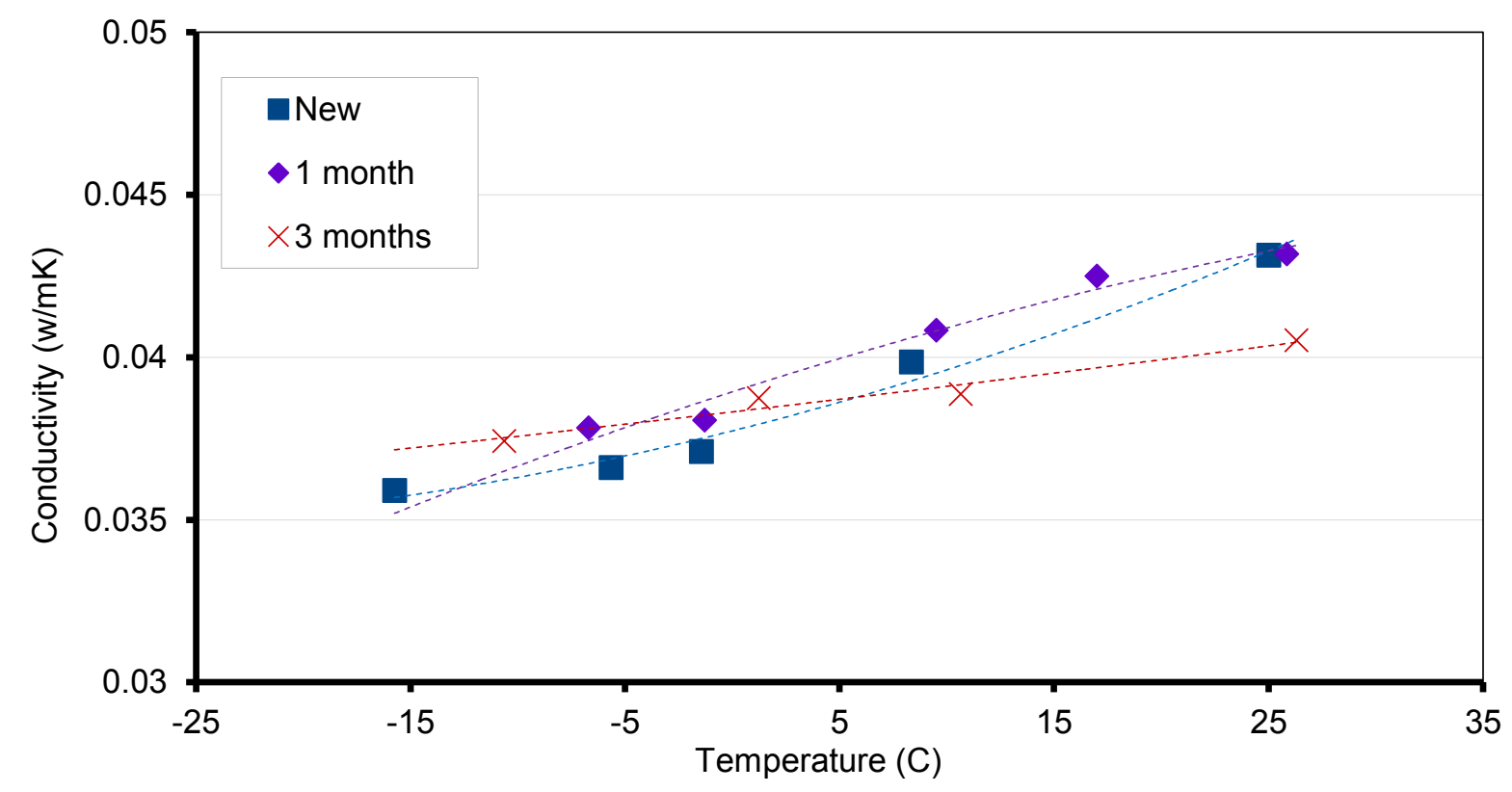

Figure 4.10: Effective Conductivity of Aged Open Cell Polyurethane (PU-C) 


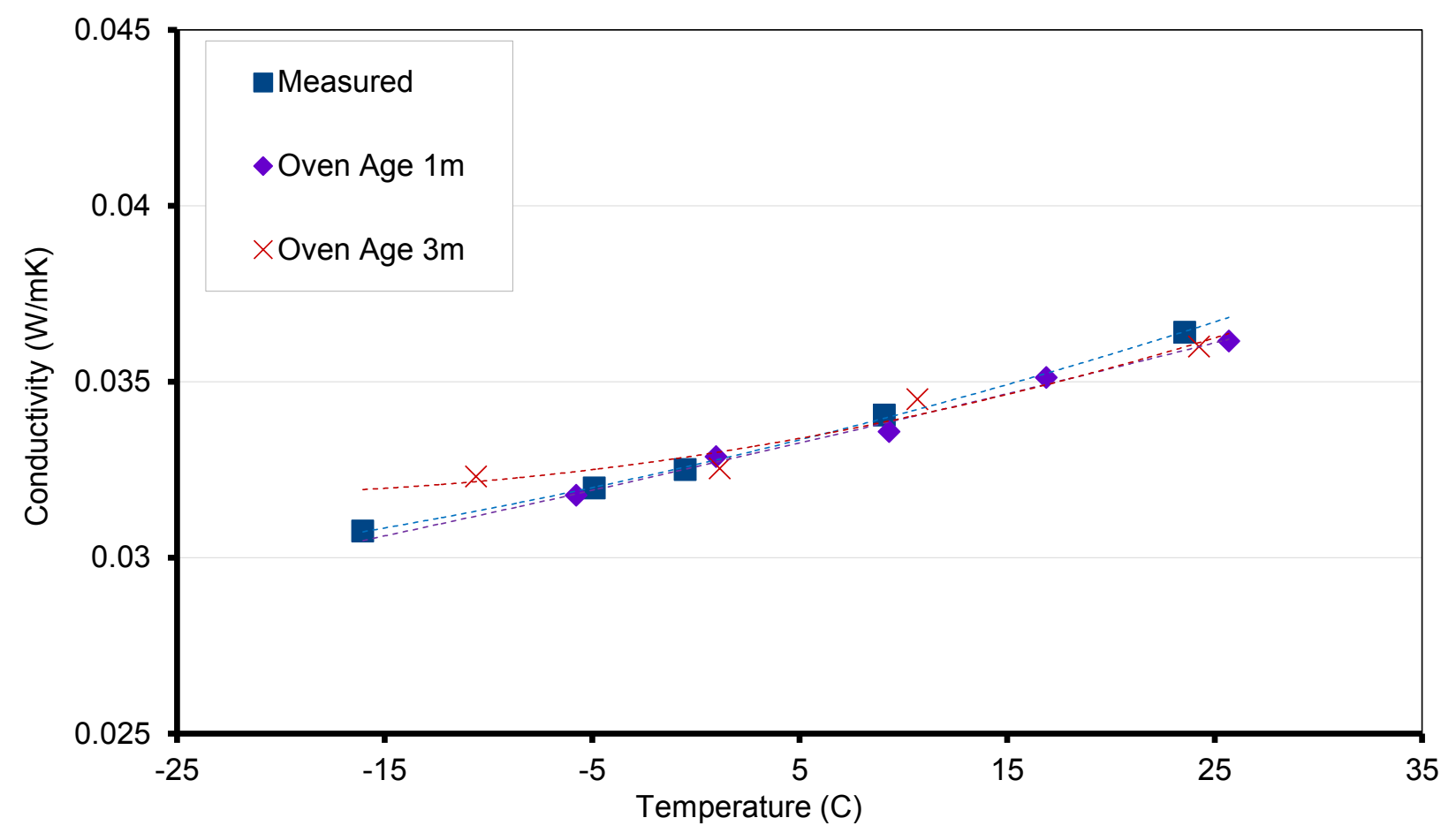

Figure 4.11: Effective Conductivity of Aged Open Cell Polyurethane (PU-D).

The other polyurethane materials (Fig.4.9-4.11) do not see this initial conductivity increase after the first month of aging, and in fact remain relatively close to the original measurements after aging. This is an interesting observation for PU-B (Fig. 4.9) because as a closed cell material, it was assumed that there would be a larger difference after the first month of aging as seen in PU-A. The minimal change in performance of open cell polyurethanes (Fig. 4.10-4.11) is expected, since there is no blowing agent within the cells. The lack of blowing agent in addition to open cells means that through this accelerated aging process, the material composition remains relatively stable, compared to closed cell materials that experience outside air infiltration as well as the escape of blowing agents. 


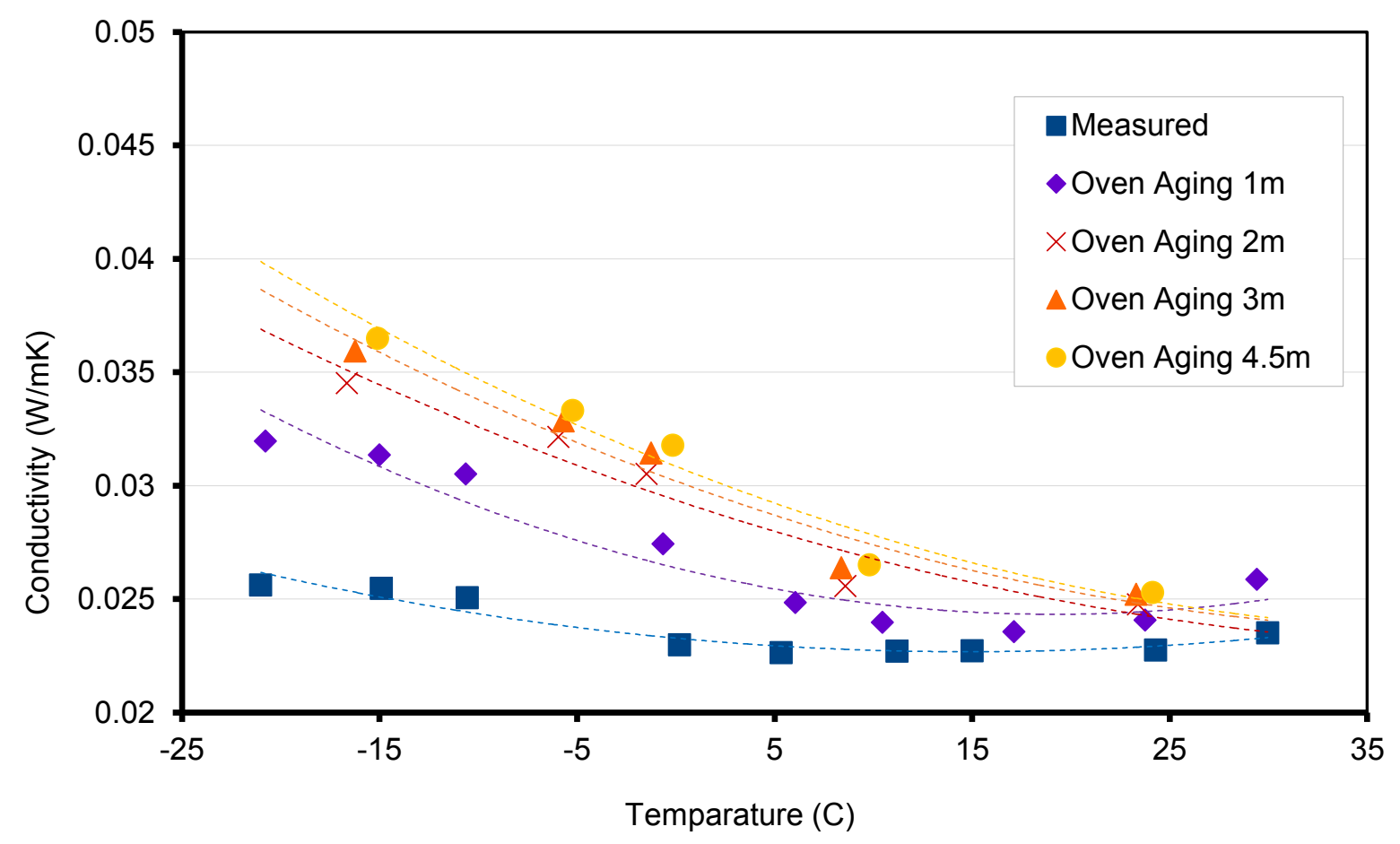

Figure 4.12: Effective Conductivity of Aged Closed Cell Polyisocyanurate (PI-A).

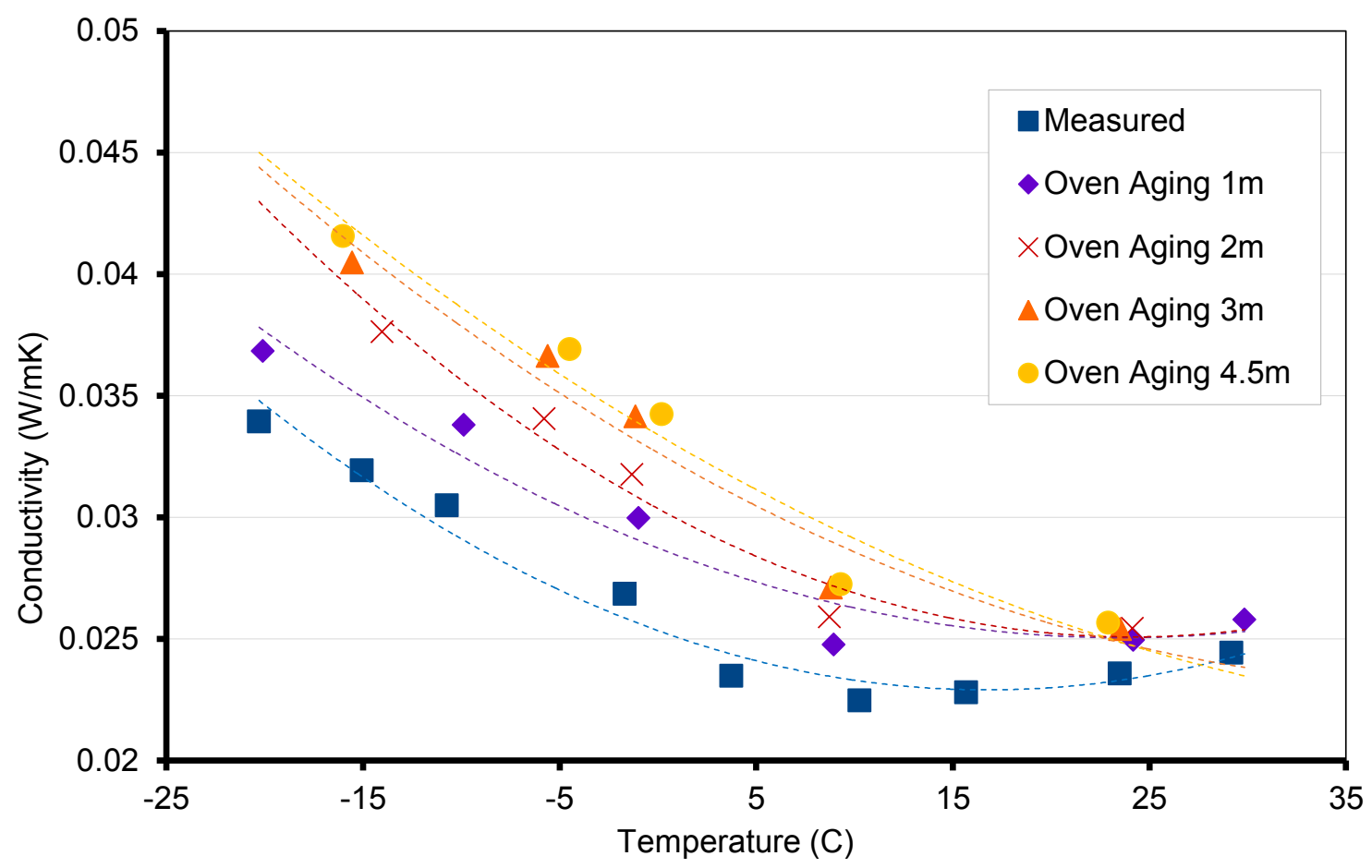

Figure 4.13: Effective Conductivity of Aged Closed Cell Polyisocyanurate (PI-B). 
In the polyisocyanurate materials (Fig. 4.12-4.13), it was observed initially that there is an evident increase in the conductivity at low temperatures in non-aged material. Interestingly, this effect appears to become even greater after aging has occurred. An important note to take from the aging of polyisocyanurate is that the difference in performance between a pristine and aged sample when measured at $24^{\circ} \mathrm{C}$ in accordance with LTTR measurements is far smaller than the difference in performance after aging at $60^{\circ} \mathrm{C}$. This means that even considering the LTTR in advertised values for polyisocyanurate materials, the actual performance may be far worse than suggested with over $40 \%$ difference in conductivity between $-15^{\circ} \mathrm{C}$ and $24^{\circ} \mathrm{C}$ after aging. 


\subsection{Moisture Results}

Table 4.1: Material Mass (g) at Varying RH\% Levels

\begin{tabular}{lcccccc}
\hline \multirow{2}{*}{ Material } & \multicolumn{7}{c}{ RH level } \\
\cline { 2 - 7 } & Dry & $40 \%$ & $80 \%$ & $90 \%$ & $95 \%$ & Submerged \\
\hline PU-A & 116.7 & 117.5 & 118.5 & 119.3 & 122.6 & 140.0 \\
PU-B & 160.3 & 162.9 & 163.8 & 165.5 & 167.9 & 212.5 \\
PU-C & 25.8 & 26.3 & 26.8 & 27.68 & 36.5 & 234.0 \\
PU-D & 60.3 & 61.1 & 61.7 & 64.5 & 69.6 & 167.0 \\
PI-A & 80.7 & 81.3 & 82.8 & 83.9 & 85.6 & 96.0 \\
PI-B & 162.3 & 164.4 & 170.8 & 185.1 & 196.8 & 213.5 \\
\hline
\end{tabular}

In Table 2, the mass of the samples measured after exposure to a range of moisture levels are reported.

The water content between dry samples and $95 \% \mathrm{RH}$ exposure are fairly significant, with the closed cell polyurethane materials both absorbing approximately $5 \%$ of their mass, open cell PU-C absorbing $44 \%$ of its mass, PU-D absorbing just over $15 \%$ of its mass, PI-A absorbing $6 \%$ of its mass, and PI-B absorbing up to $\sim 21 \%$ of its mass, though the cardboard facing on the sample was responsible for absorbing a larger portion of the moisture. After full submersion, the samples all absorbed a significant amount of water, with the open cell polyurethanes expectedly increasing by the greatest overall percentage of the original dried weight, due to the sponge like nature of the materials. While the closed cell samples were more resistant to absorption, both polyurethane and polyisocyanurate samples still absorbed a relatively large amount of water after submersion. At each of the moisture set points, the materials were also measured for conductivity across the same range of temperatures as previous measurements. These results are shown in figures $4.14-4.19$. From these graphs, some similarities between the same types of materials were seen. In both closed cell polyurethanes, the effect of moisture is relatively minimal on the conductivity. In open cell materials, major spikes in conductivity were seen at the highest moisture levels only, with an increased effect at higher temperatures. For the polyisocyanurate materials, changes in effective conductivity are seen across each moisture set point, with increased effects at low temperatures. 


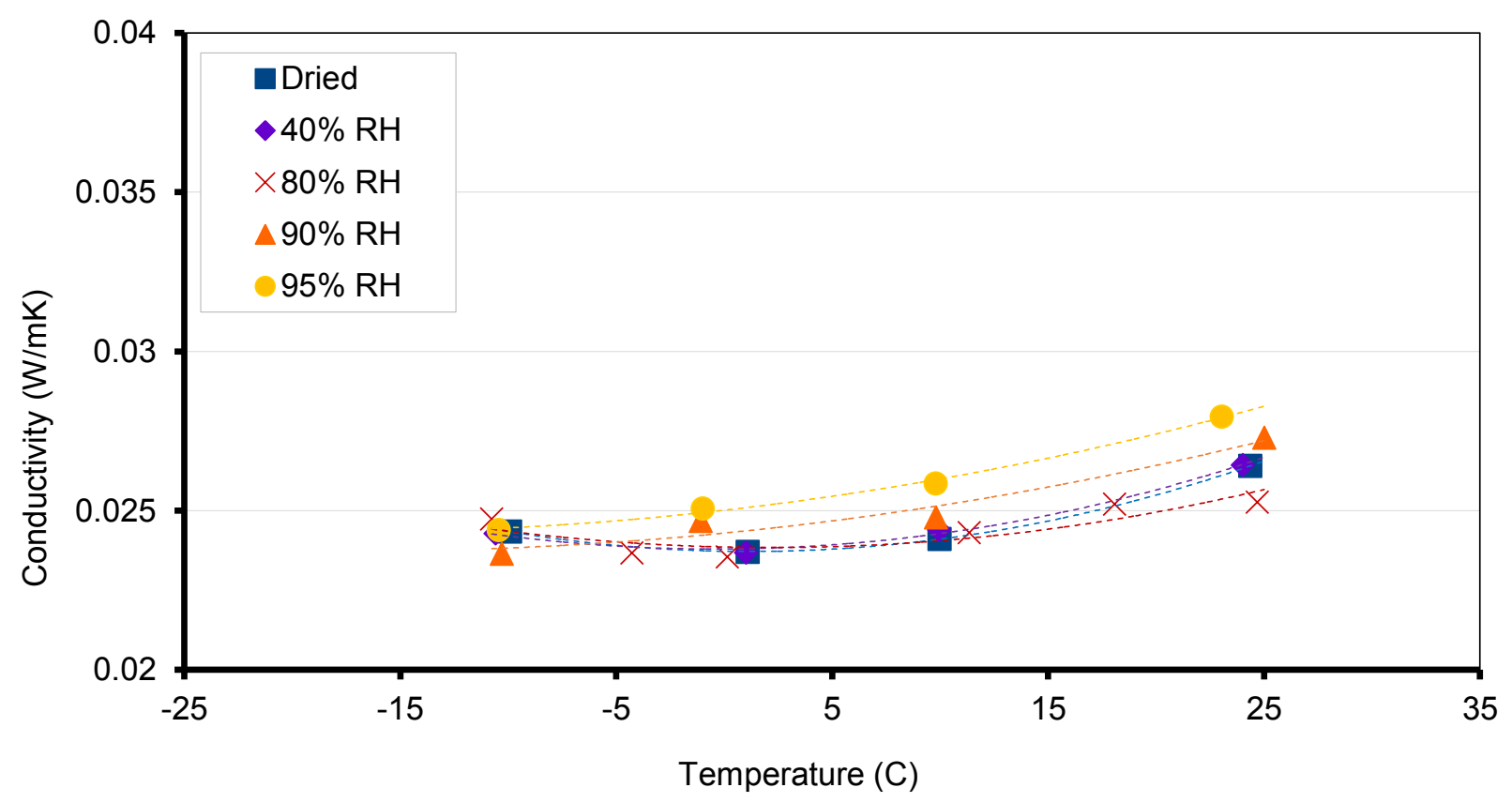

Figure 4.14: Effective Conductivity of Closed Cell Polyurethane A across a range of moisture levels

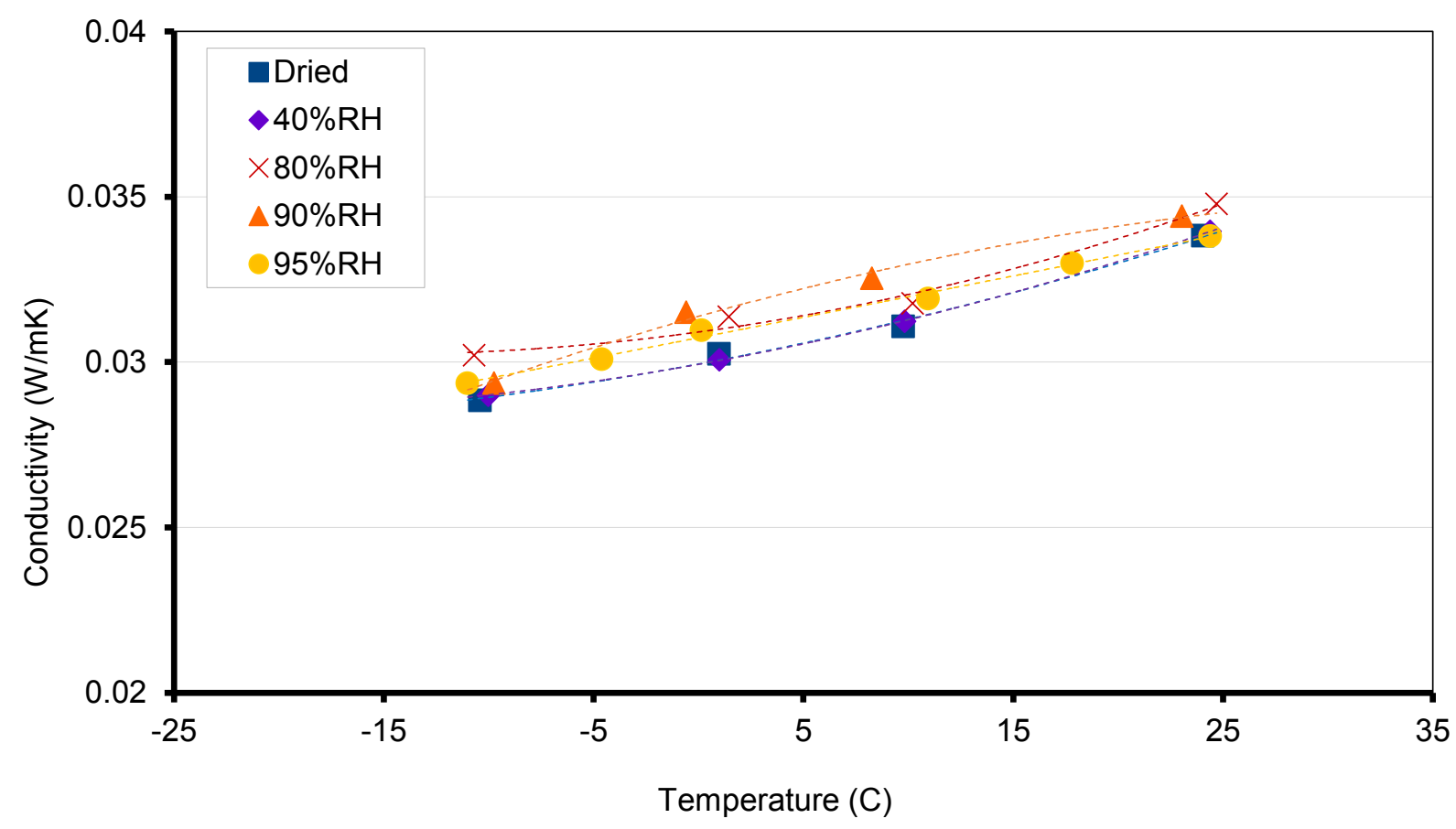

Figure 4.15: Effective Conductivity of Closed Cell Polyurethane B across a range of moisture levels 
As indicated in Fig. 4.14-4.15, the closed cell polyurethane materials are affected slightly by exposure to high moisture levels. In both materials, it was measured that at the higher moisture levels above $90 \%$ $\mathrm{RH}$, there is approximately $5-10 \%$ increase in conductivity from the dried samples. The difference in performance over a range of moisture levels is small compared to the other types of materials. In the open cell polyurethane materials, major increases in conductivity were measured at higher moisture levels. In PU-C (Fig. 4.16), it can be seen that between a dried state and exposure to $90 \% \mathrm{RH}$, there is around $8-12 \%$ increase in conductivity over a range of temperatures. After exposure to $95 \% \mathrm{RH}$, the sample was affected to a very high degree because of increased moisture content, especially at higher temperatures. While PU-D does not see such a massive effect at $95 \% \mathrm{RH}$, the sample still experiences an increase in conductivity of $12-50 \%$ over a range of temperatures between a dried state and exposure to humidity levels above $90 \%$, indicating that the open cell samples are heavily influenced by moisture levels. This can be expected due to the nature of open cell materials, allowing more free movement of moisture into the material, becoming relatively more saturated at high humidity levels.

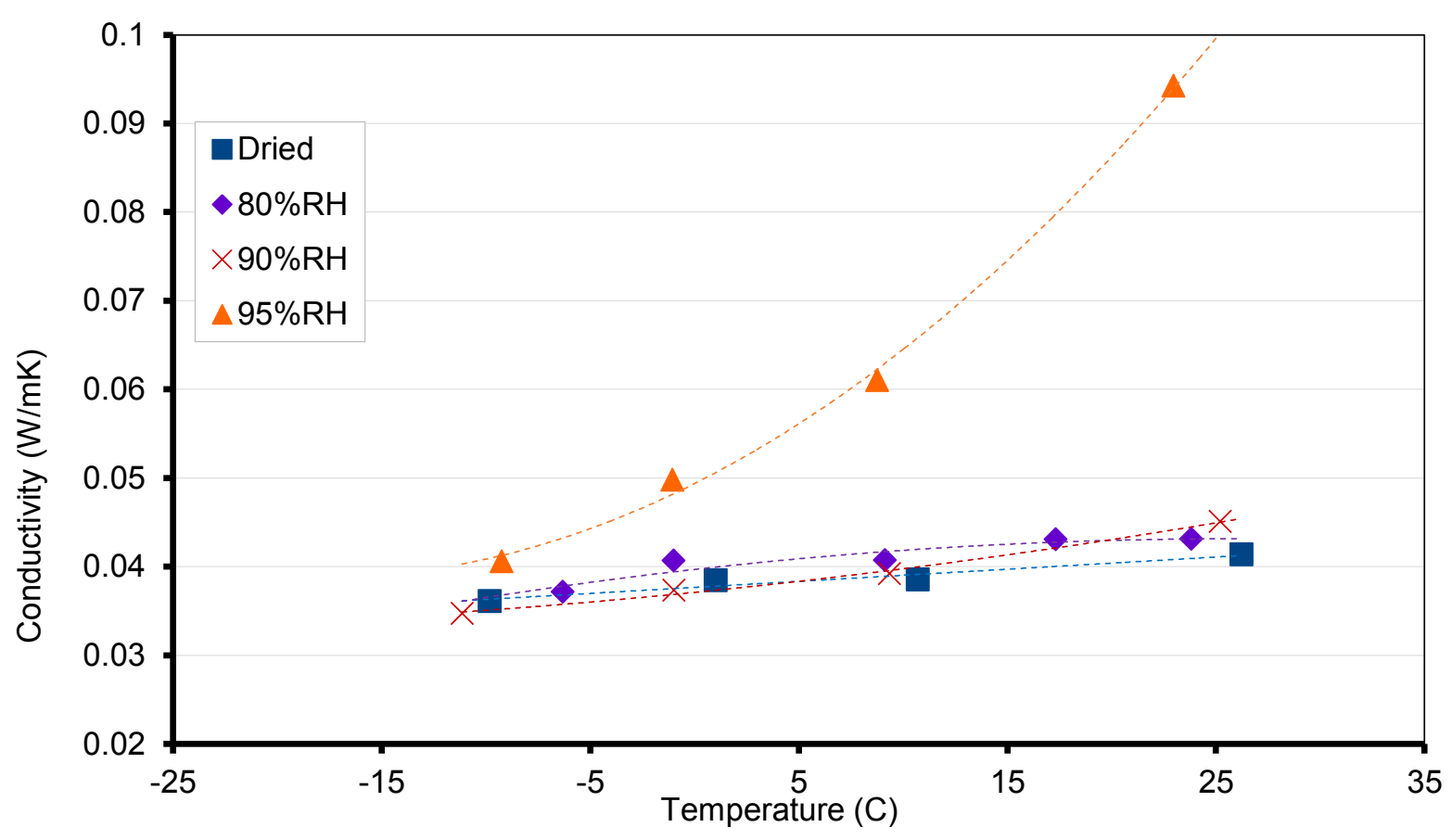

Figure 4.16: Effective Conductivity of Open Cell Polyurethane C across a range of moisture levels 


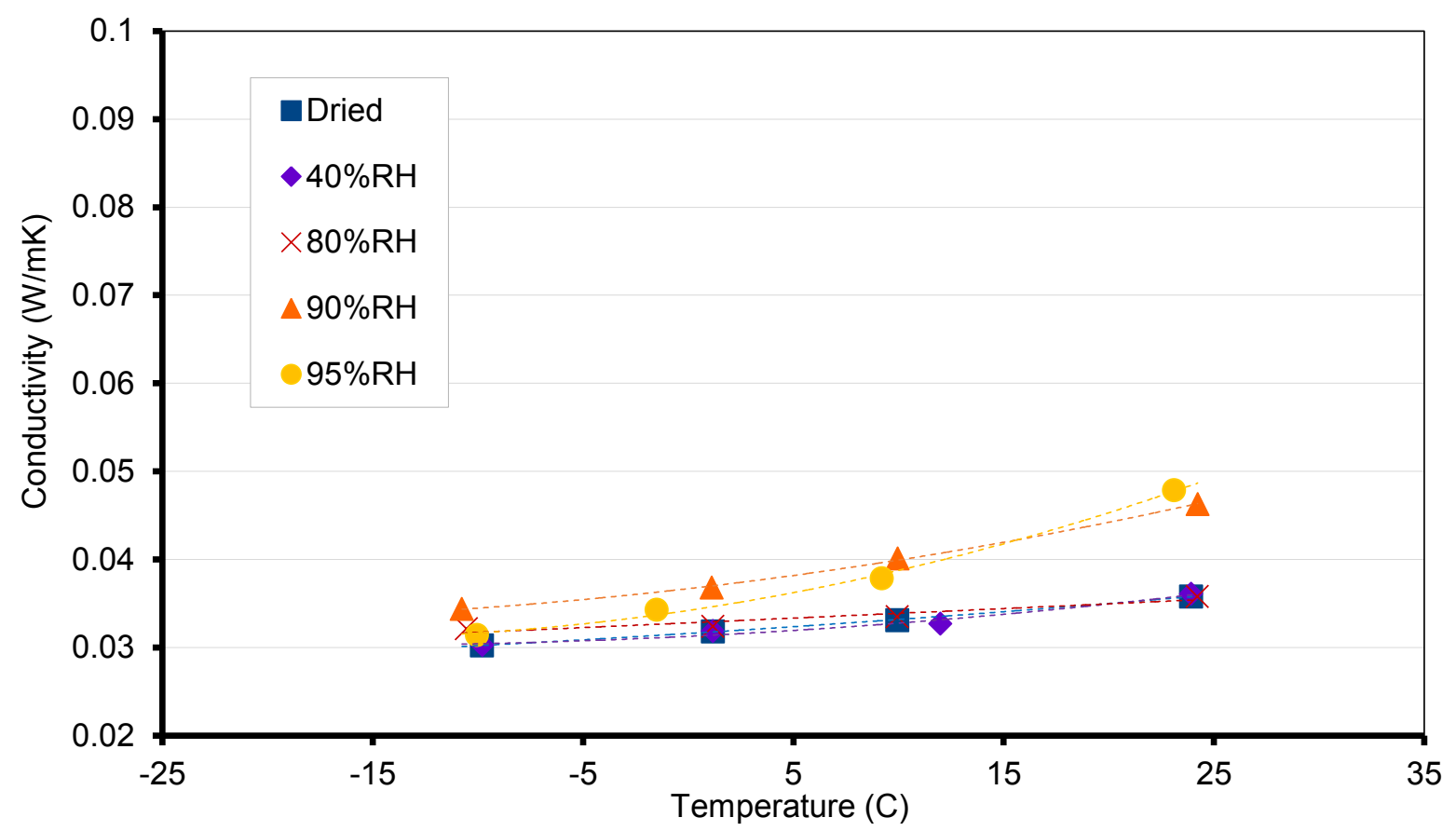

Figure 4.17: Effective Conductivity of Open Cell Polyurethane D across a range of moisture levels

In the polyisocyanurate materials (Fig 4.18-4.19), a very interesting effect was measured. While both samples experience an increase in conductivity of around $10 \%$ at the higher end of the temperature range, at low temperatures, the effect of increased moisture is massive. In both materials, increases in conductivity of over $50 \%$ were measured. When looking at a set of temperature dependent conductivity curves measured over a range of moisture levels, the idea of effective conductivity becomes even clearer. With a full understanding of how environmental conditions will affect the performance of the materials, it becomes increasingly clear that a singular value for material conductivity cannot be trusted. 


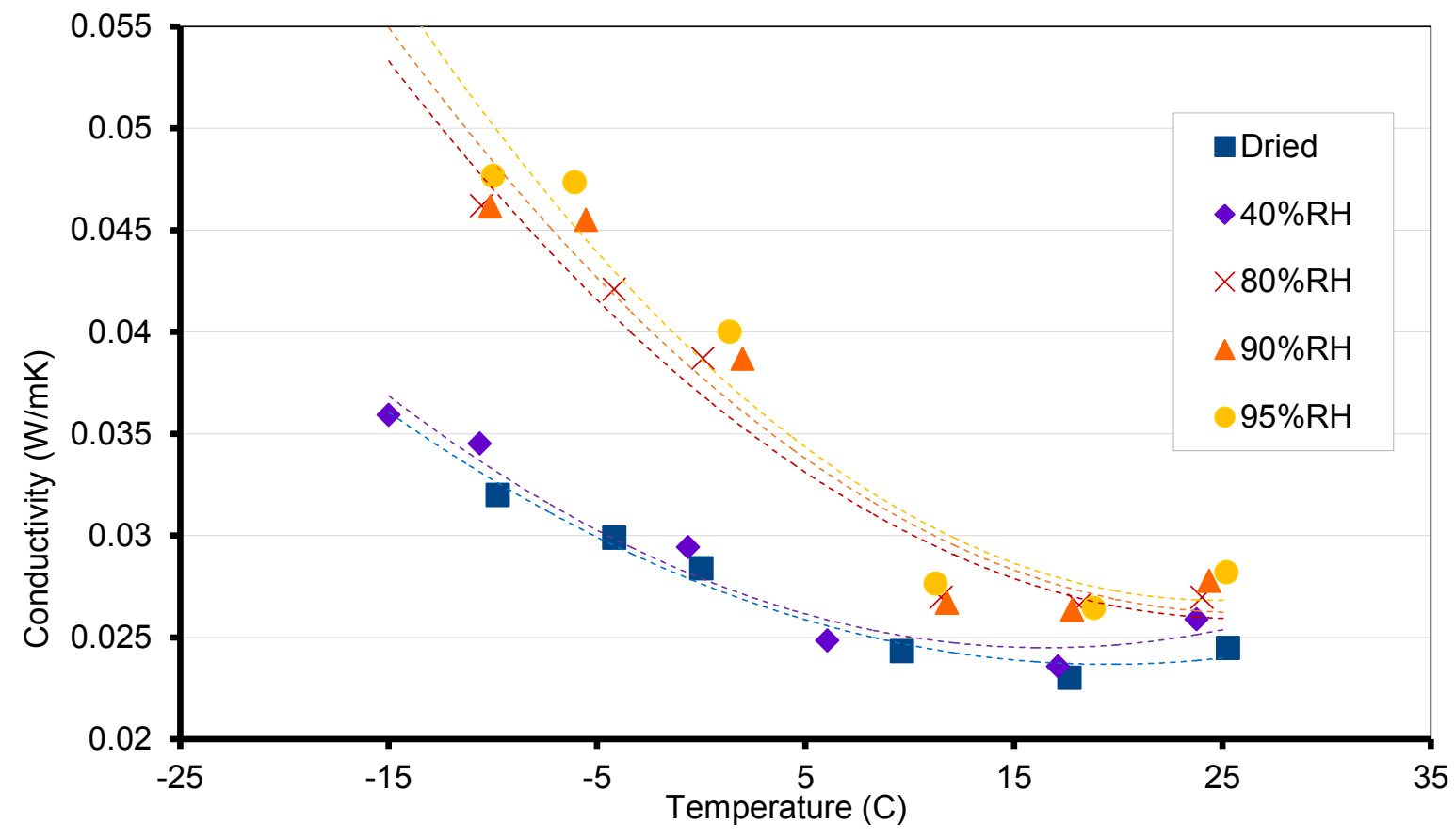

Figure 4.18: Effective Conductivity of Closed Cell Polyisocyanurate A across a range of moisture level

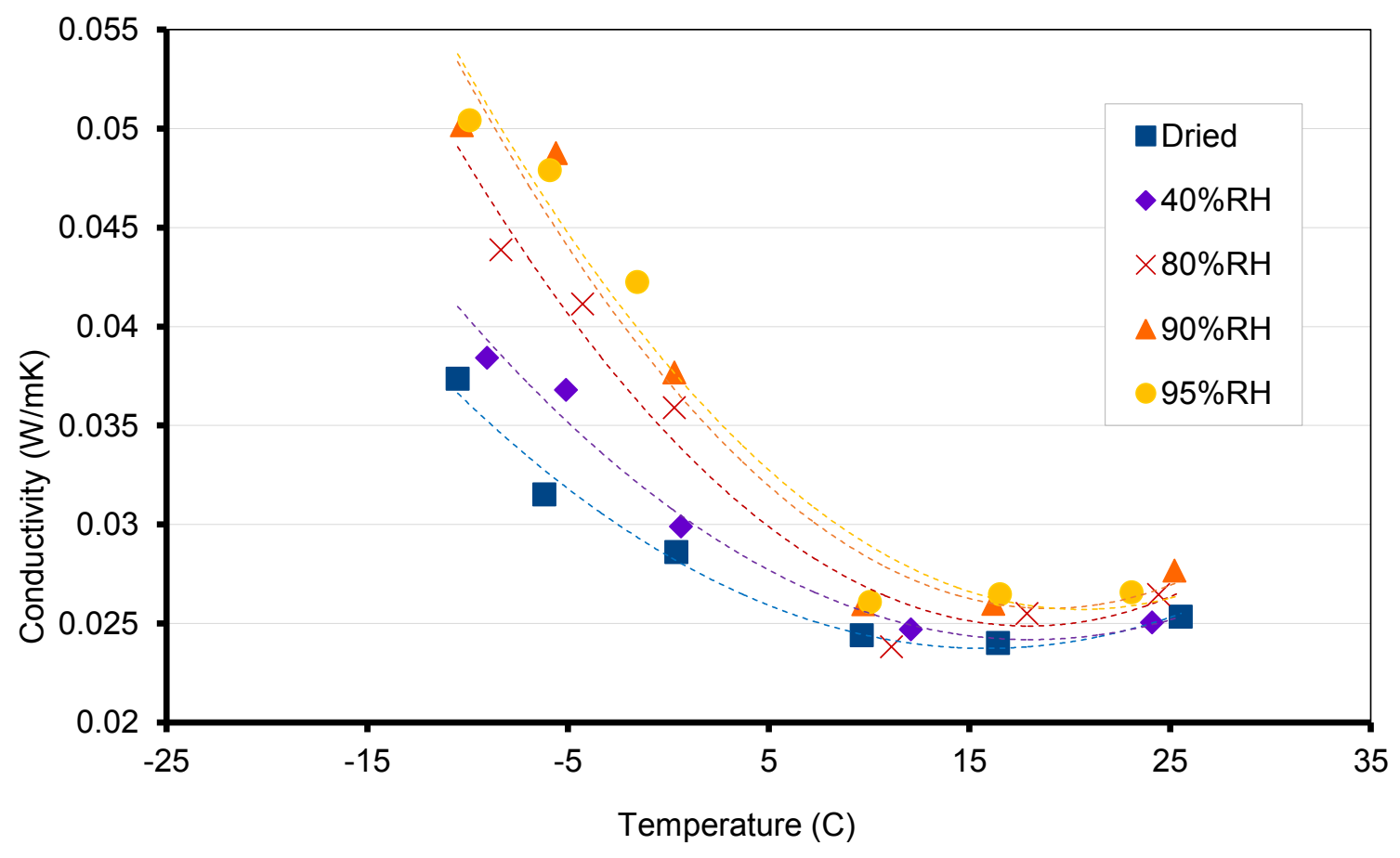

Figure 4.19: Effective Conductivity of Closed Cell Polyisocyanurate B across a range of moisture level 


\subsection{Freeze-Thaw Results}

In this section, both the effective conductivity and moisture storage properties were measured for the materials after undergoing freeze-thaw cycling as described in the methodology. In the measured conductivity results over 150 freeze-thaw cycles, shown in Figs $4.20-4.25$, the materials show results similar to what was seen in the heat accelerated aging results, though to a slightly lesser degree.

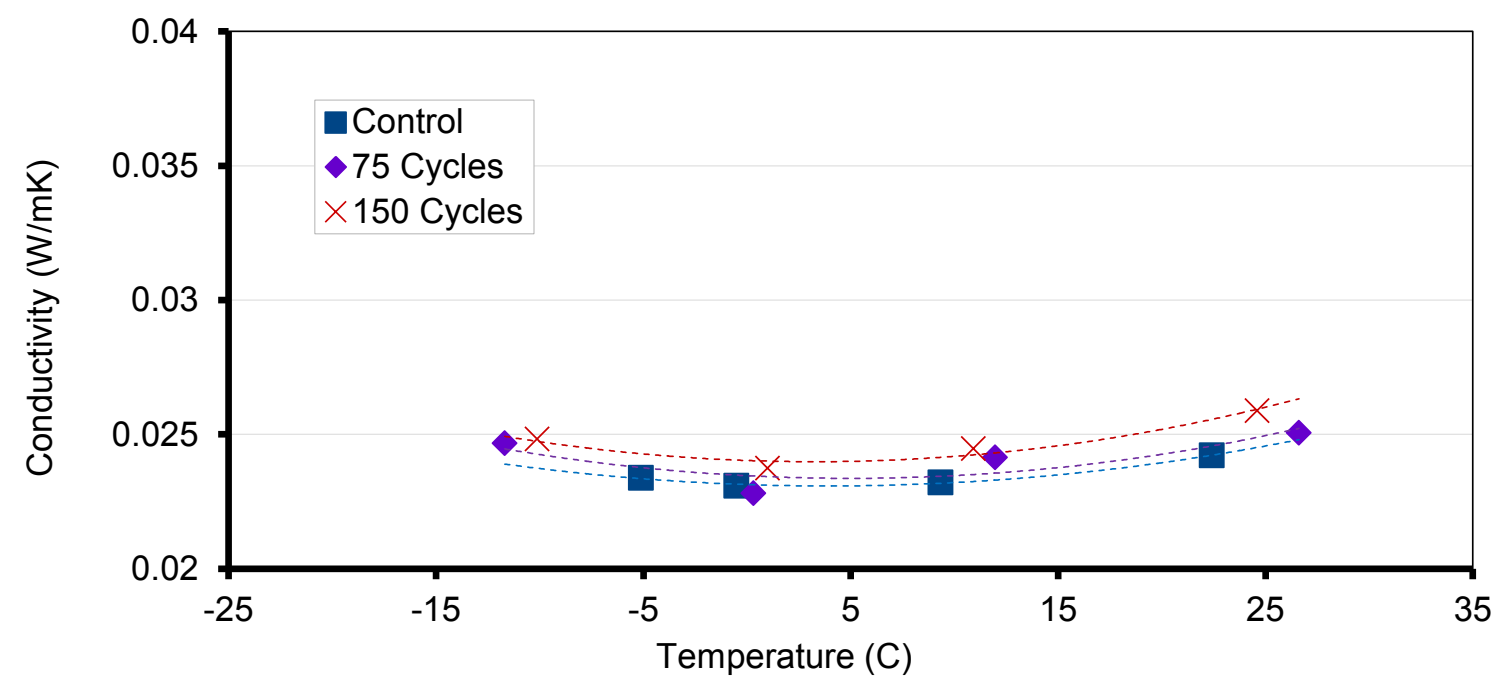

Figure 4.20: Effective Conductivity of Closed Cell Polyurethane after Freeze-Thaw Cycles (PU-A).

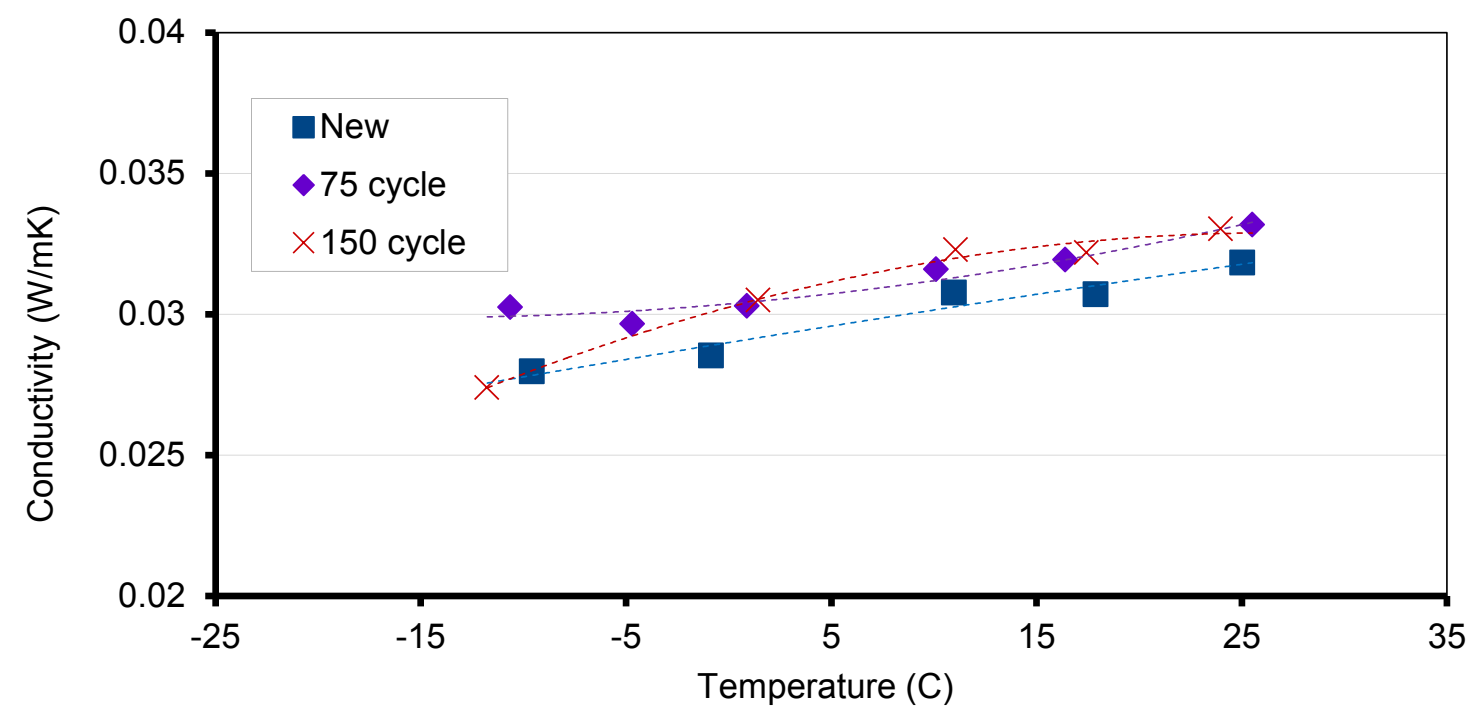

Figure 4.21: Effective Conductivity of Open Cell Polyurethane after Freeze-Thaw Cycles (PU-B). 
In the two closed cell polyurethane samples PU-A and PU-B (Fig. 4.20-4.21), the material shows a slightly higher conductivity after the freeze-thaw cycles, though still remaining relatively stable compared to the changes seen in other test methods. The open cell materials PU-C and PU-D have a relatively consistent conductivity over the freeze-thaw cycling.

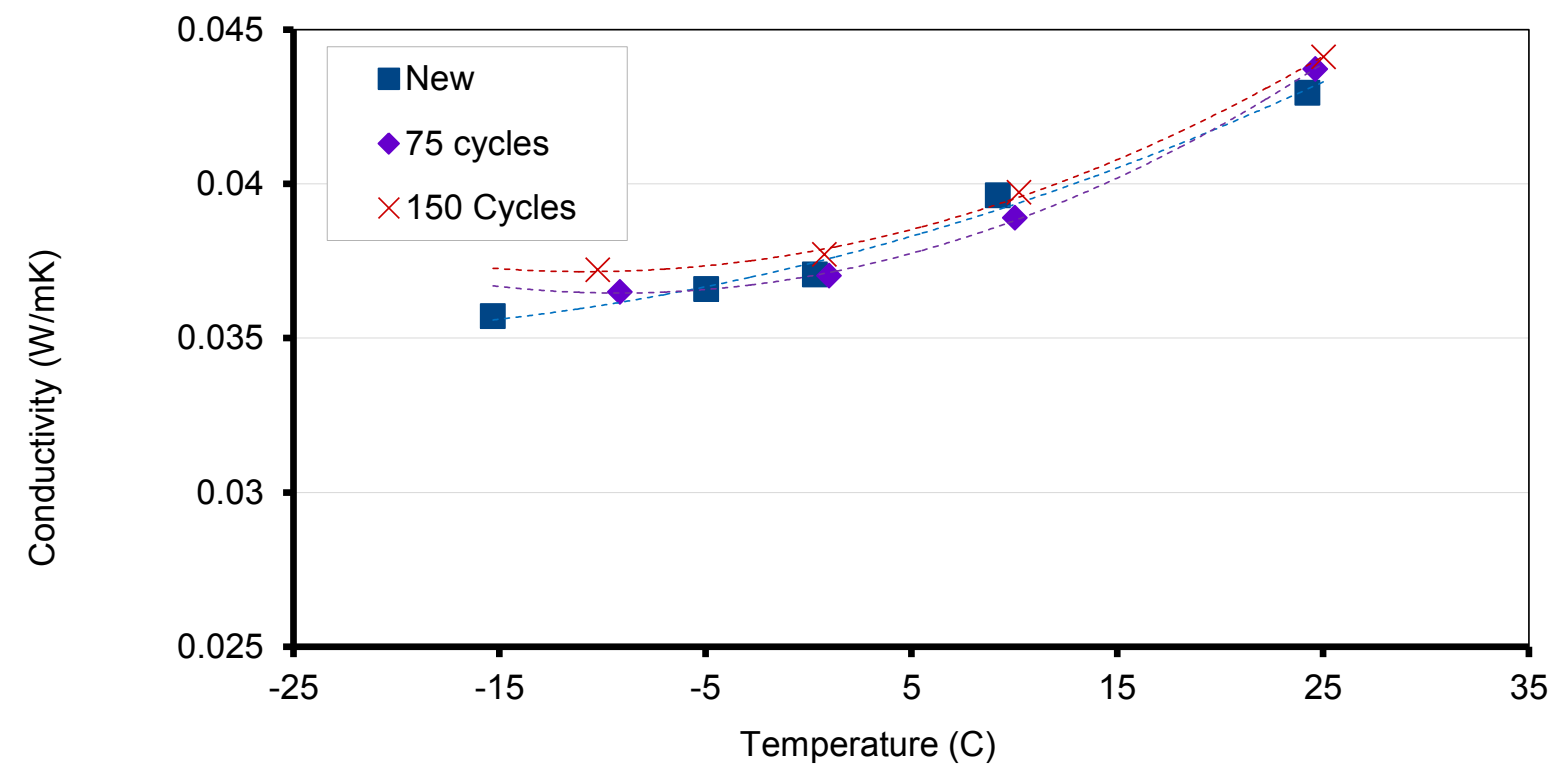

Figure 4.22: Effective Conductivity of Open Cell Polyurethane after Freeze-Thaw Cycles (PU-C).

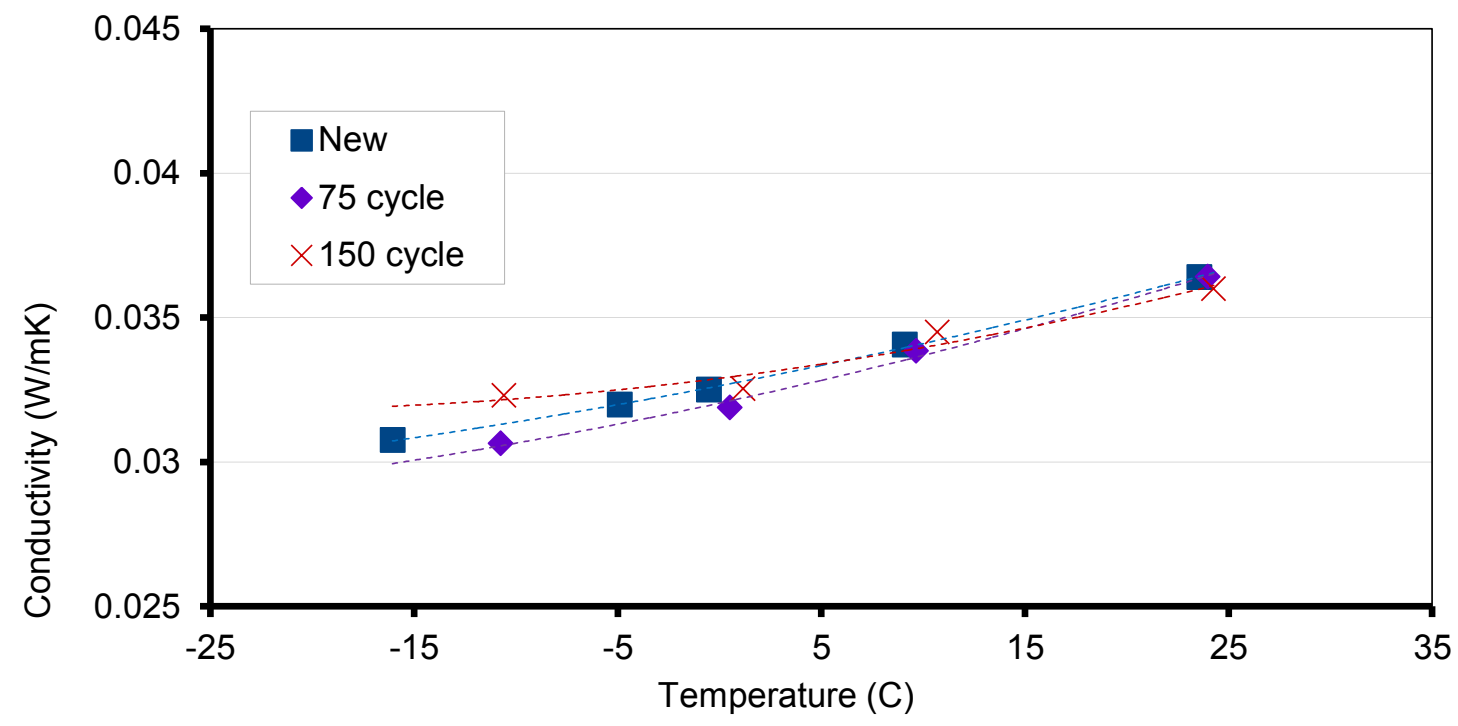

Figure 4.23: Effective Conductivity of Open Cell Polyurethane after Freeze-Thaw Cycles (PU-D). 
In the polyisocyanurate materials PI-A and PI-B, the trend is again similar to the results of elevated temperature aging. In both materials, it is seen that the effects of the freeze-thaw cycling have a larger impact on the performance at cold temperatures. This indicates that similarly to heat accelerated aging, this aging method causes some of the insulating blowing agent to escape and outside air to infiltrate the materials.

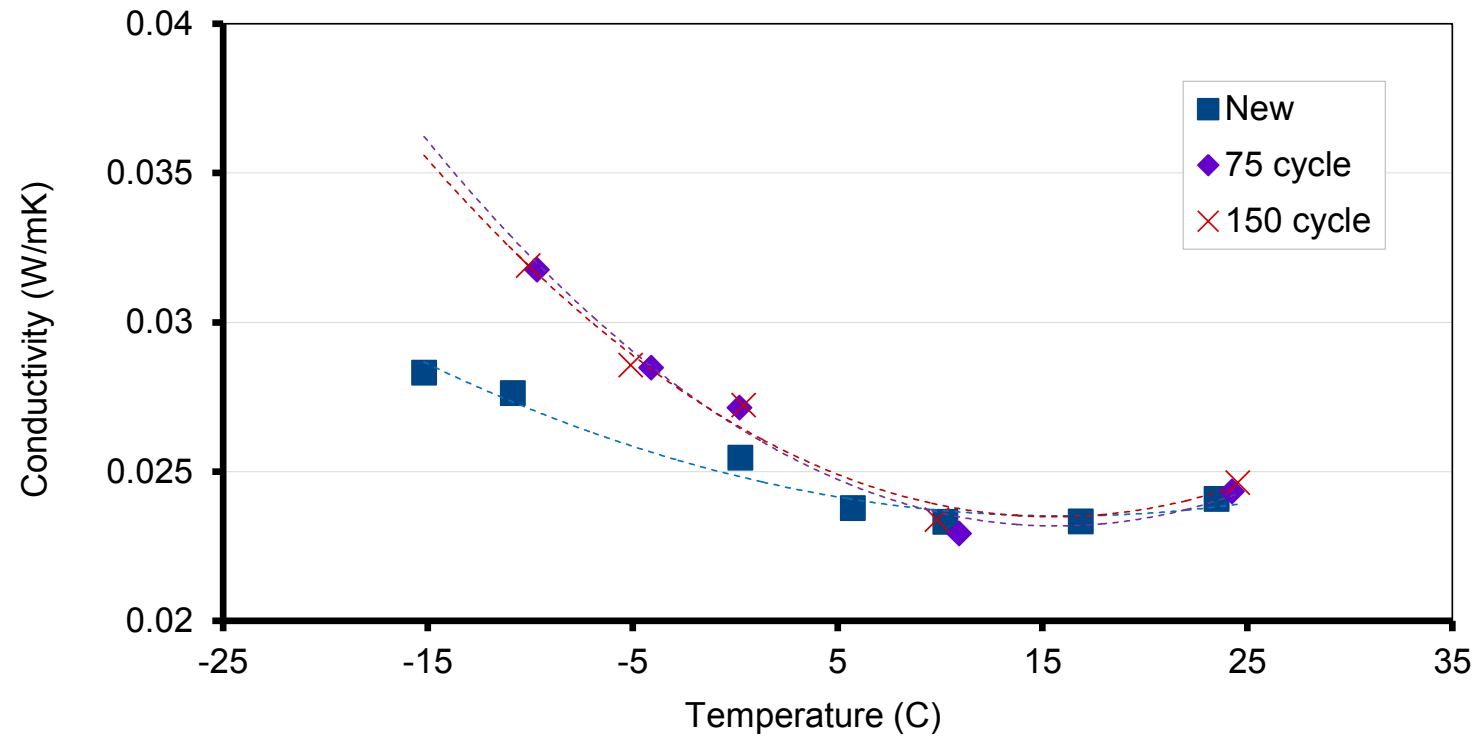

Figure 4.24: Effective Conductivity of Closed Cell Polyisocyanurate after Freeze-Thaw Cycles (PI-A).

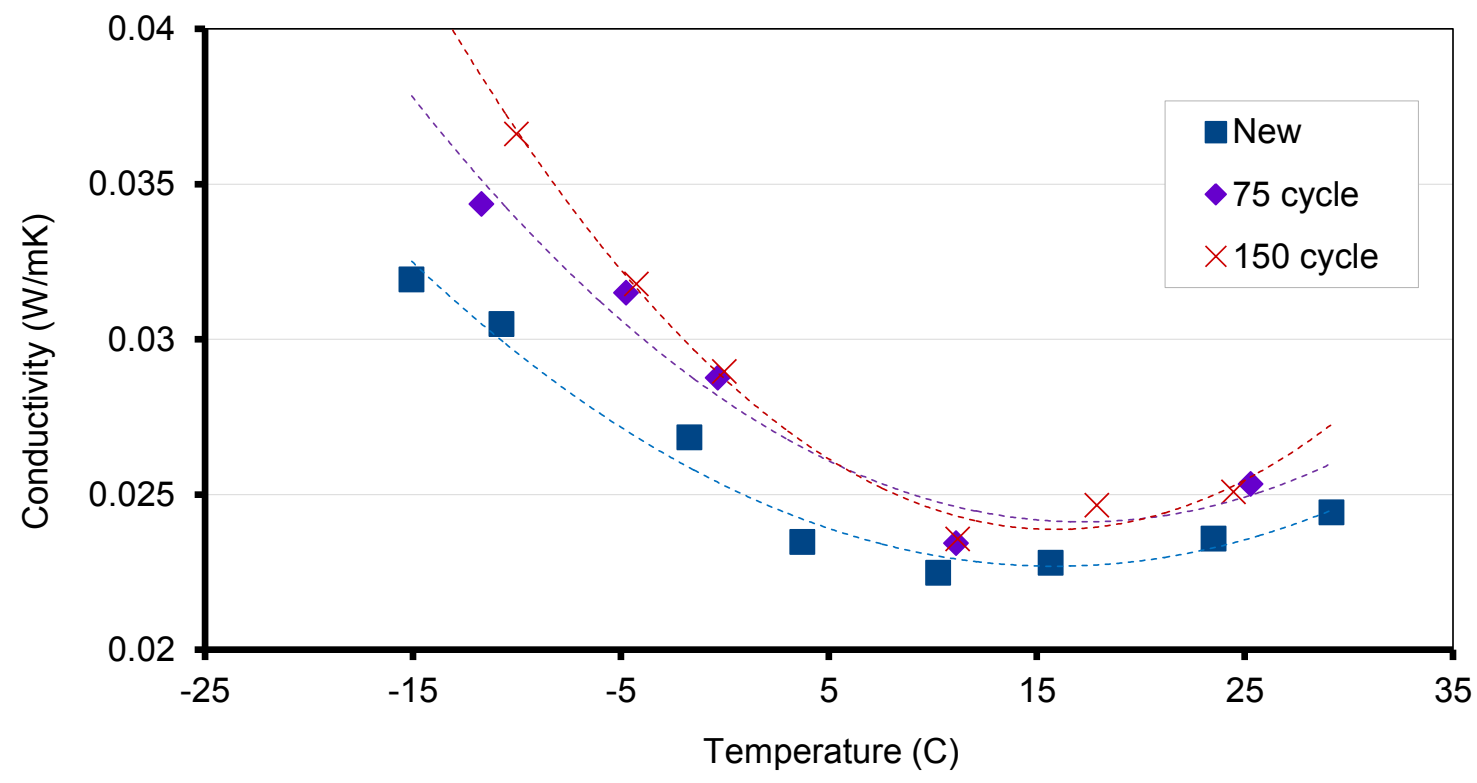

Figure 4.25: Effective Conductivity of Closed Cell Polyisocyanurate after Freeze-Thaw Cycles (PI-B). 
Following 150 freeze-thaw cycles, the samples were also exposed to high moisture levels in order to compare the impact of moisture before and after freeze-thaw cycling. In Table 3, the sample mass are recorded, here it can be seen that after 150 freeze-thaw cycles, for the most part the materials behave similarly to before freeze-thaw cycling, though absorb slightly more water.

Table 4.2: Material Mass (g) at Varying RH\% Levels after 150 Freeze-Thaw Cycles

\begin{tabular}{lccccc}
\hline \multirow{2}{*}{ Material } & \multicolumn{5}{c}{ RH\% / Moisture level } \\
\cline { 2 - 6 } & Dried & $80 \%$ & $90 \%$ & $95 \%$ & Submerged \\
\hline Polyurethane A - Closed Cell & 102.3 & 104.8 & 105.5 & 113.4 & 132.5 \\
Polyurethane B - Closed Cell & 166.4 & 168.0 & 170.9 & 179.5 & 227.5 \\
Polyurethane C - Open Cell & 28.2 & 29.4 & 30.6 & 40.4 & 262.5 \\
Polyurethane D - Open Cell & 64.2 & 66.2 & 68.3 & 84.8 & 187.0 \\
Polyisocyanurate A - Alum. Faced & 81.6 & 83.4 & 84.7 & 88.4 & 99.5 \\
Polyisocyanurate B - Card. Faced & 164.4 & 176.8 & 184.0 & 197.8 & 238.5 \\
\hline
\end{tabular}

The moisture storage capabilities of the materials were seen to increase at the highest humidity levels and after submersion. In Fig 4.26-4.31, the moisture content of the material samples measured at a range of humidity levels as well as full submersion are displayed as a percentage of the original samples dried weight. These graphs compare the moisture storage capabilities of the material samples that were experienced freeze-thaw cycling to samples that were only exposed to high moisture levels. It was shown that especially after submersion, the materials that were exposed to freeze-thaw cycling were able to absorb slightly more water content. The implication of this effect is that after experiencing many freeze-thaw cycles in a Canadian climate, humidity levels may further affect material conductivity through more moisture absorbed at the same humidity levels are before freeze-thaw cycling. 


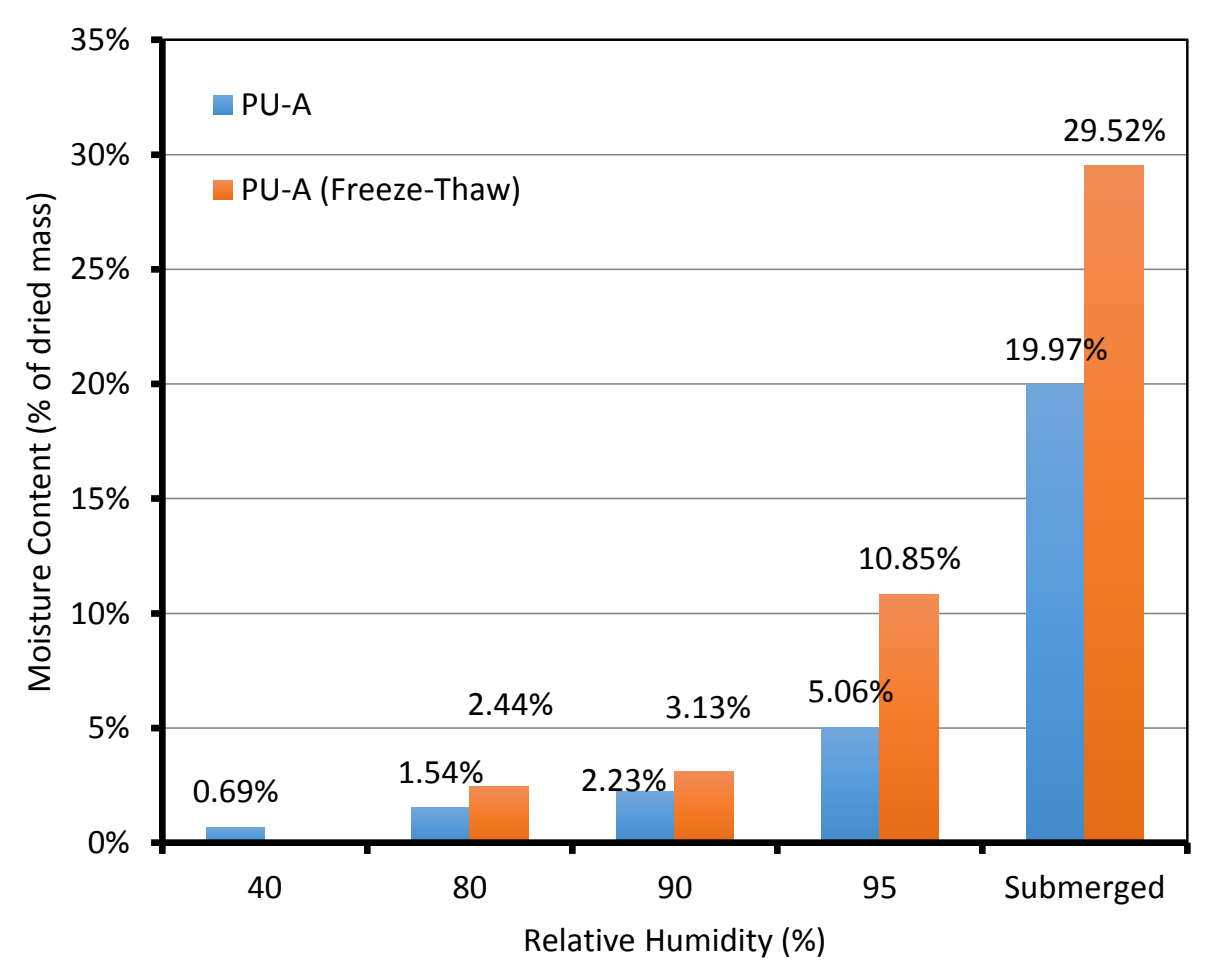

Figure 4.26: Moisture Content in closed cell polyurethane over a range of moisture levels (PU-A)

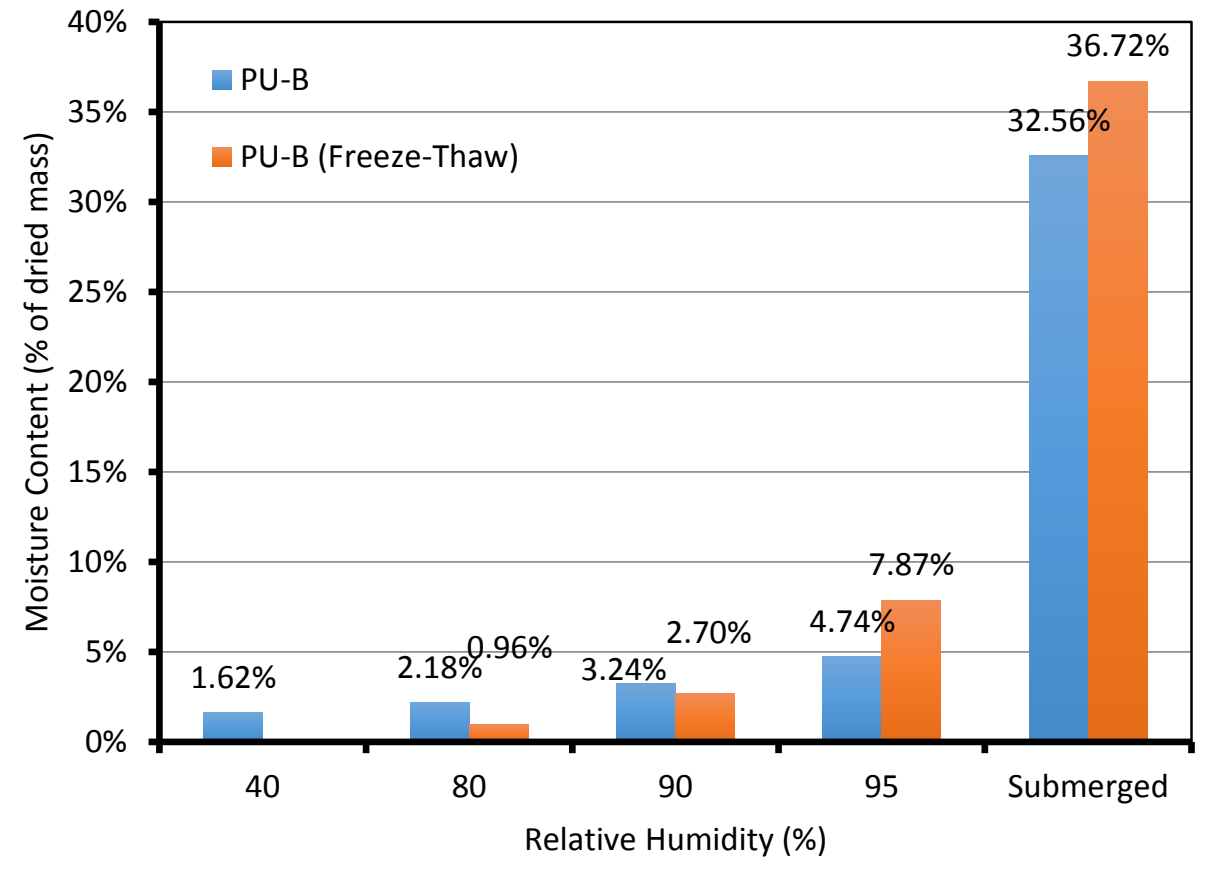

Figure 4.27: Moisture Content in closed cell polyurethane over a range of moisture levels (PU-B) 

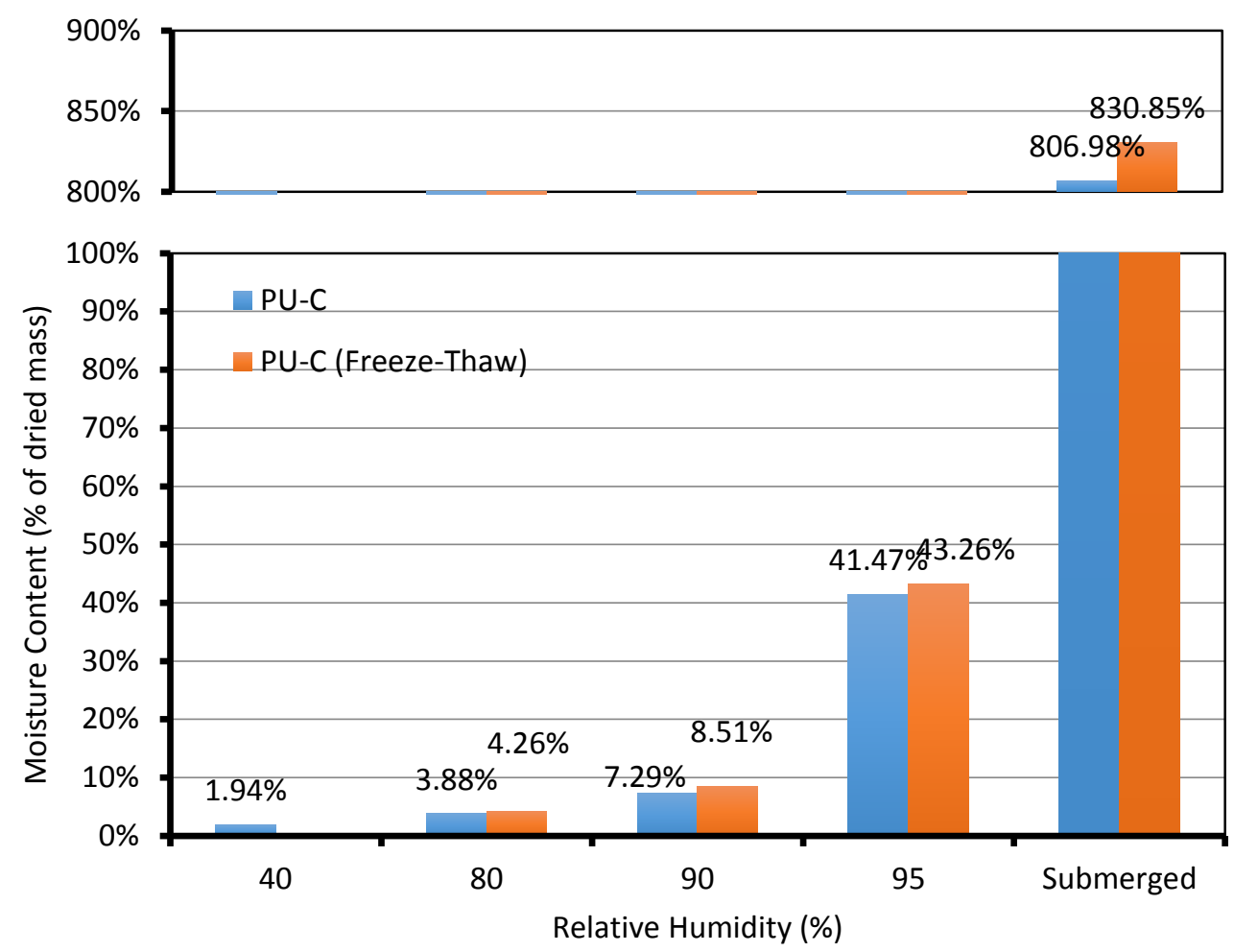

Figure 4.28: Moisture Content in open cell polyurethane over a range of moisture levels (PU-C)

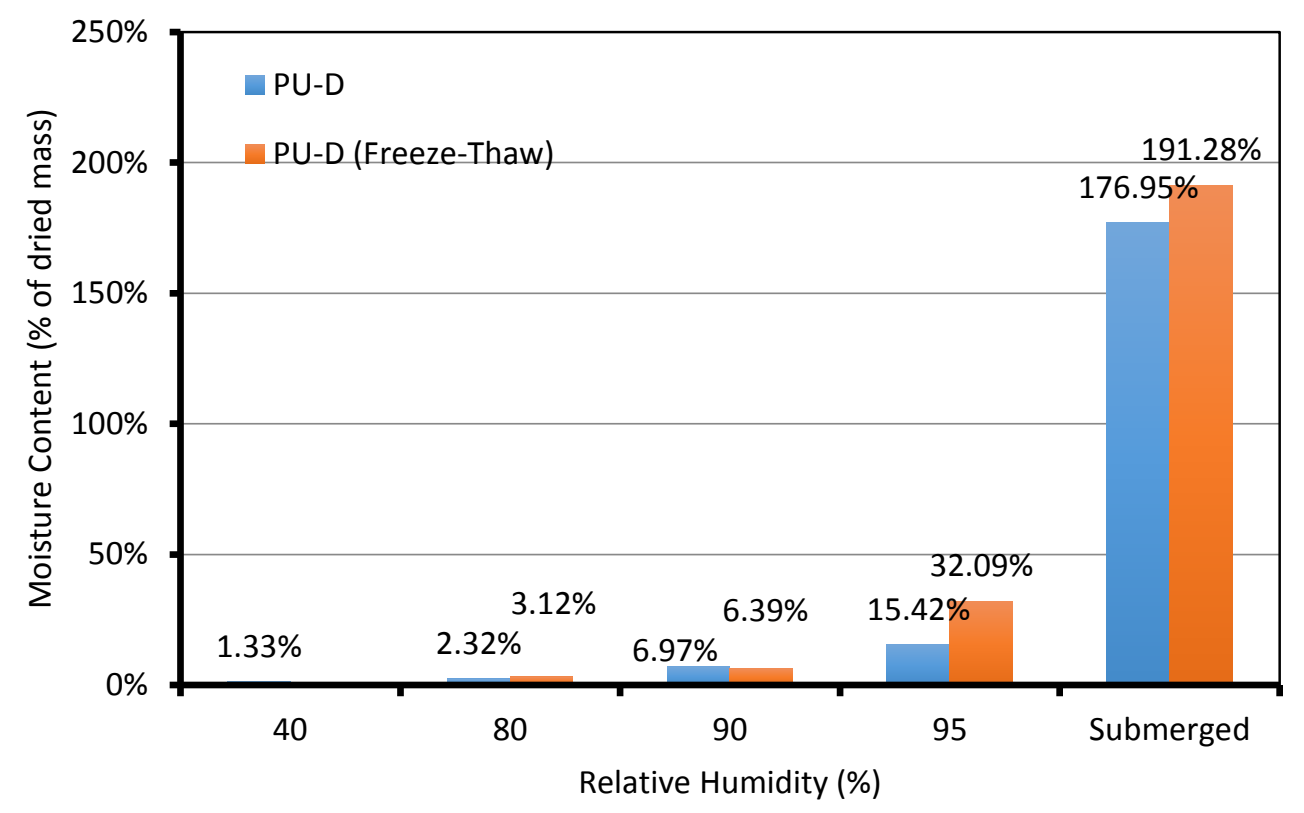

Figure 4.29: Moisture Content in open cell polyurethane over a range of moisture levels (PU-D) 


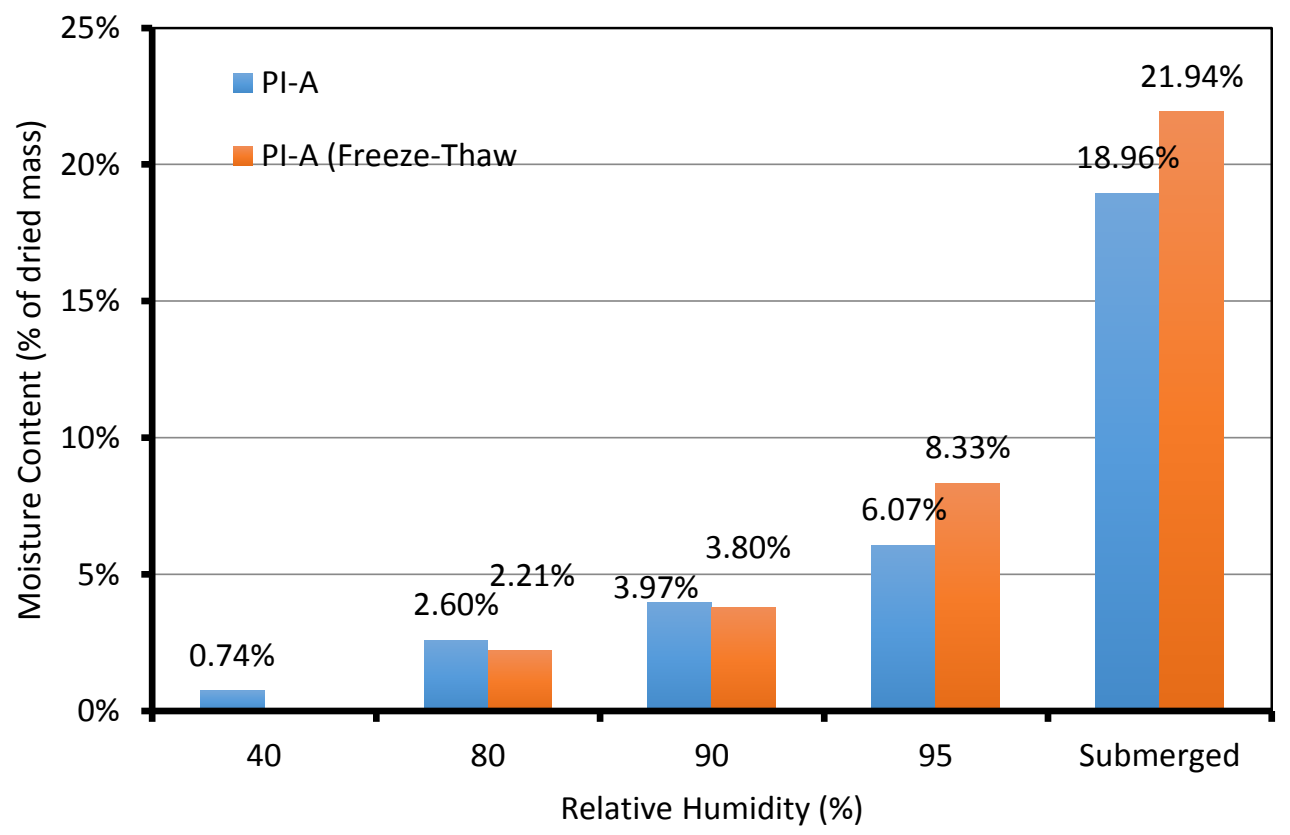

Figure 4.30: Moisture Content in closed cell polyisocyanurate over a range of moisture levels (PI-A)

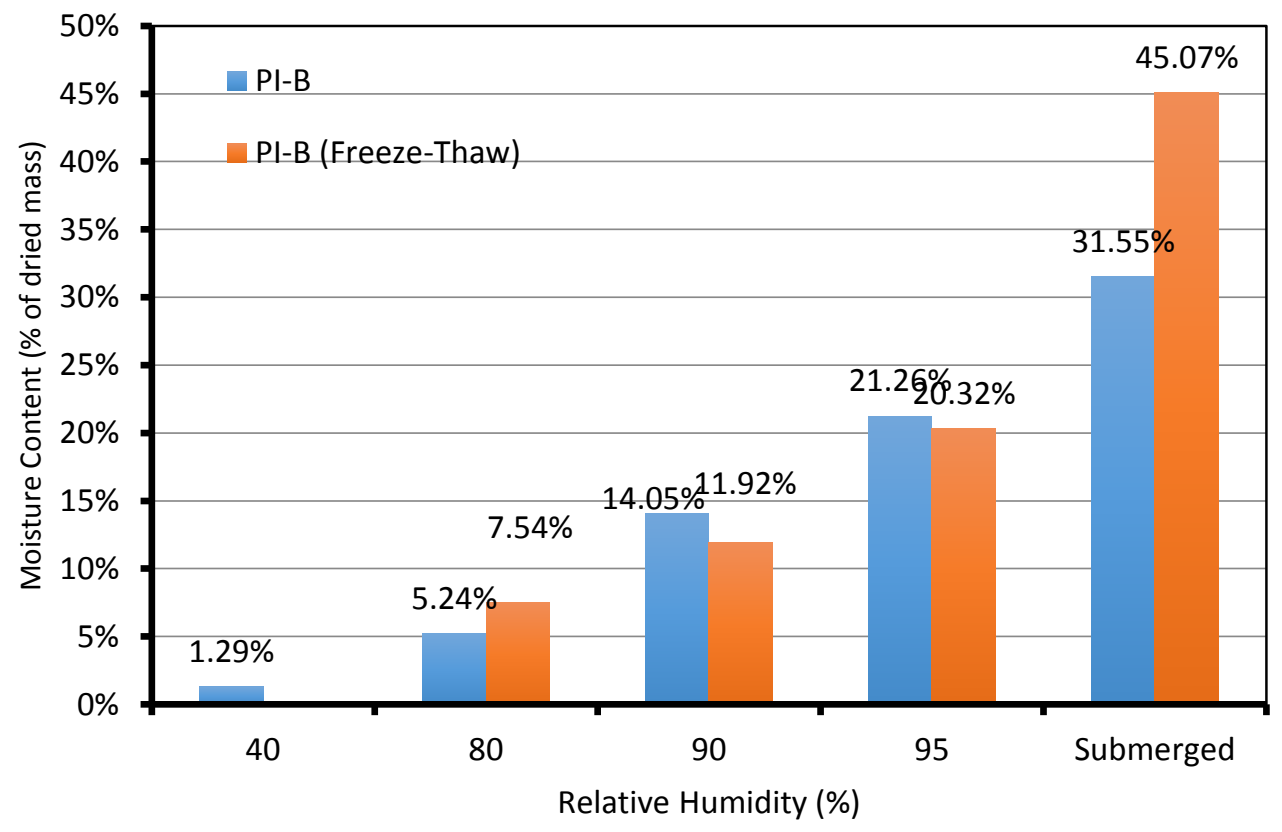

Figure 4.31: Moisture Content in closed cell polyisocyanurate over a range of moisture levels (PI-B) 
At the highest humidity levels, the samples absorbed slightly more moisture than before freezethaw cycling, however as a result, the increased moisture absorption had minimal effect on the material performance. Measured conductivity values are very close to the results of samples exposed to moisture alone. From the results in Fig 4.32-4.37, it can be seen that the trends measured at high moisture levels after freeze-thaw cycling are very similar to the results of moisture exposure alone. This indicates that while the freeze-thaw cycling allowed slightly more moisture to be absorbed at higher humidity levels, that increase had minimal effects on the actual performance.

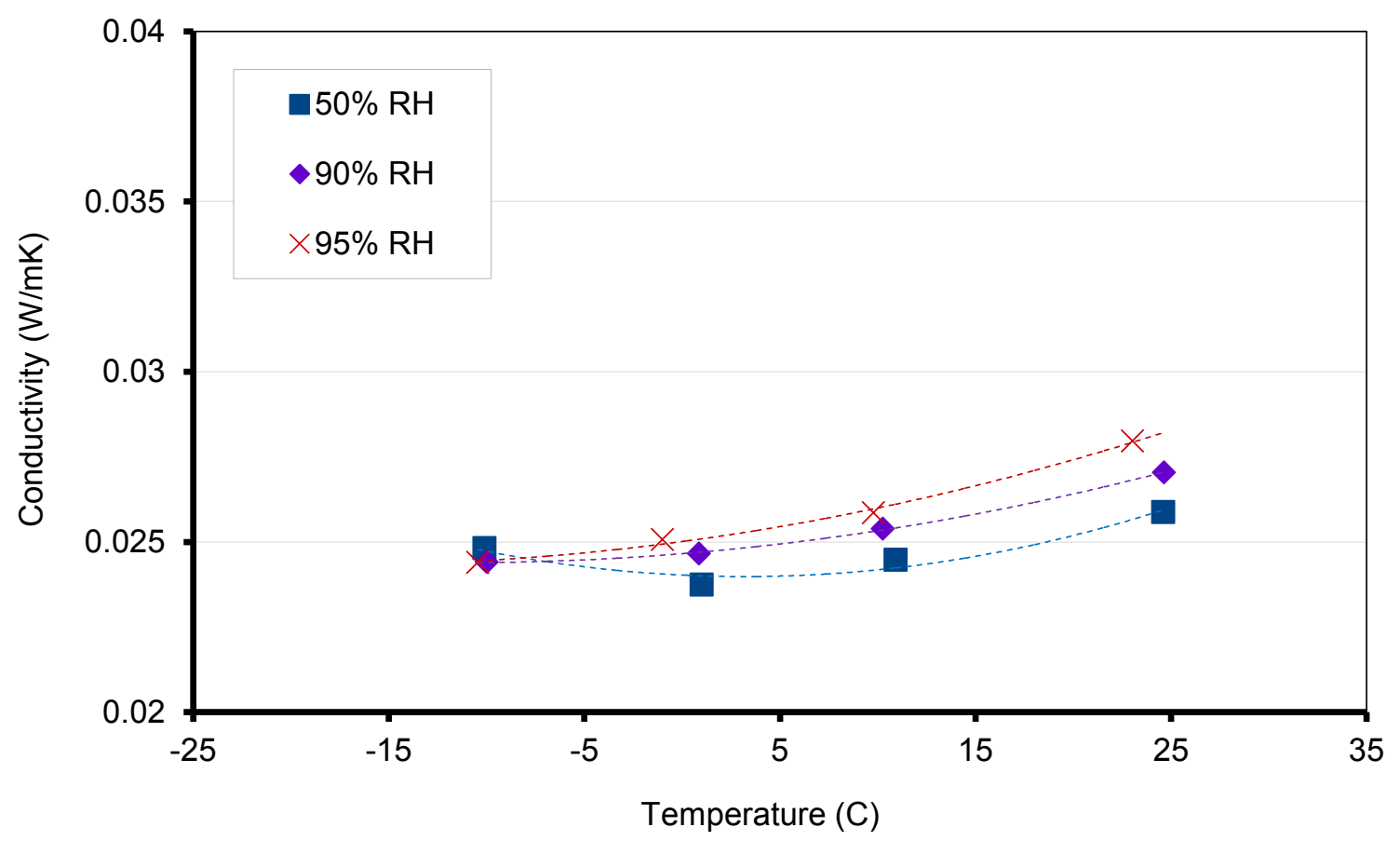

Figure 4.32: Effective Conductivity of Closed Cell Polyurethane after 150 Freeze-Thaw Cycles and Moisture Exposure (PU-A). 


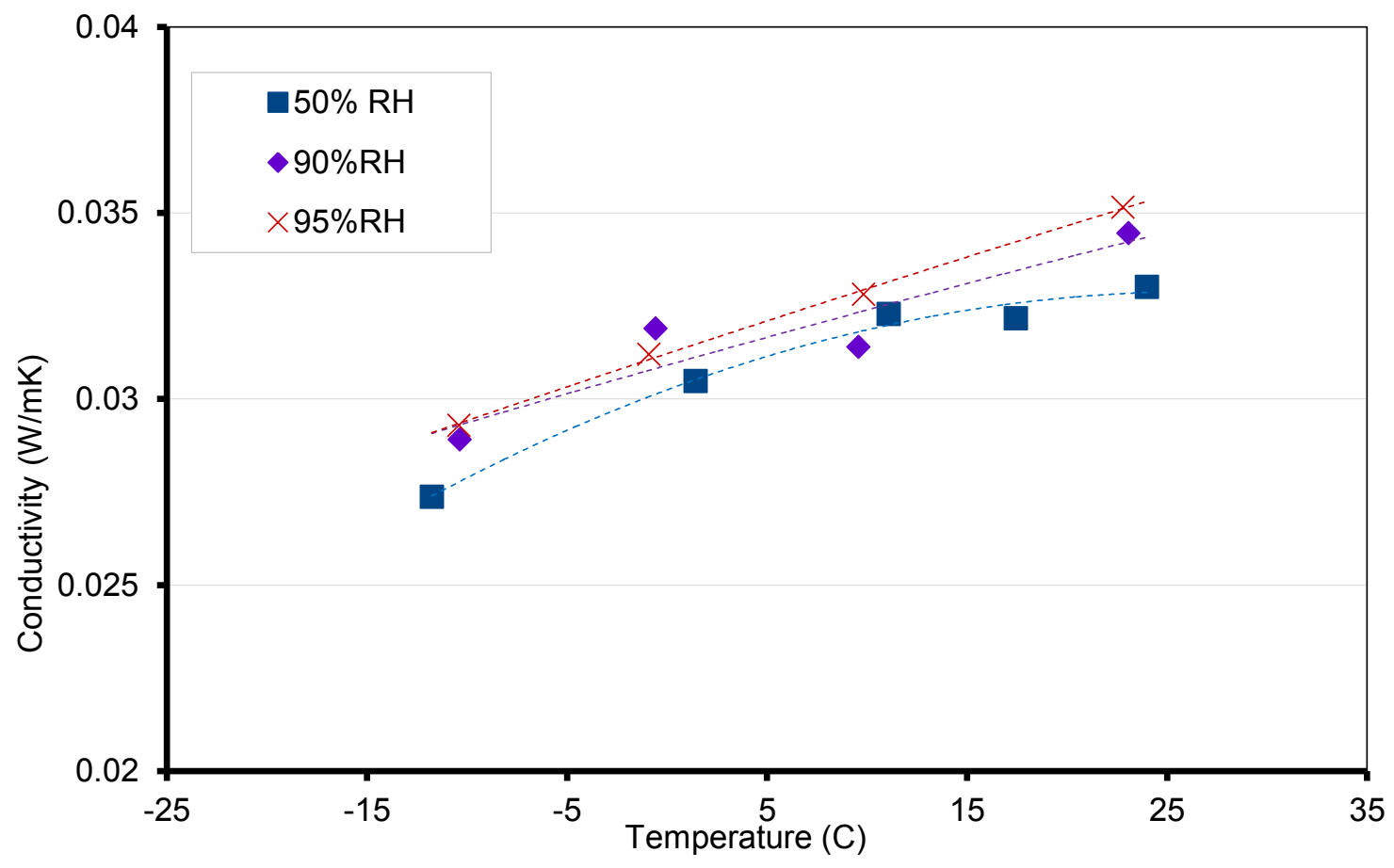

Figure 4.33: Effective Conductivity of Closed Cell Polyurethane after 150 Freeze-Thaw Cycles and Moisture Exposure (PU-B).

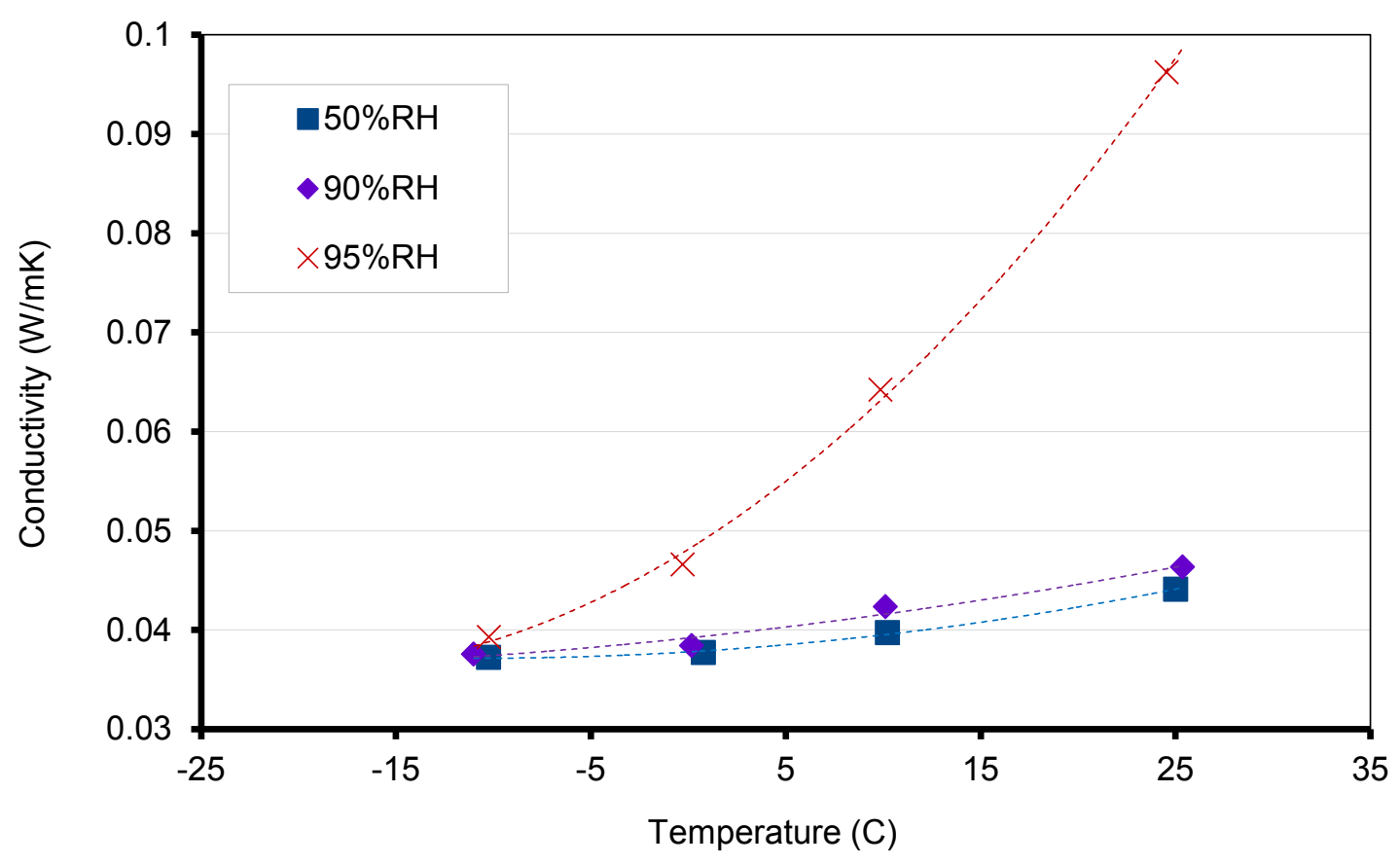

Figure 4.34: Effective Conductivity of Open Cell Polyurethane after 150 Freeze-Thaw Cycles and Moisture Exposure (PU-C). 


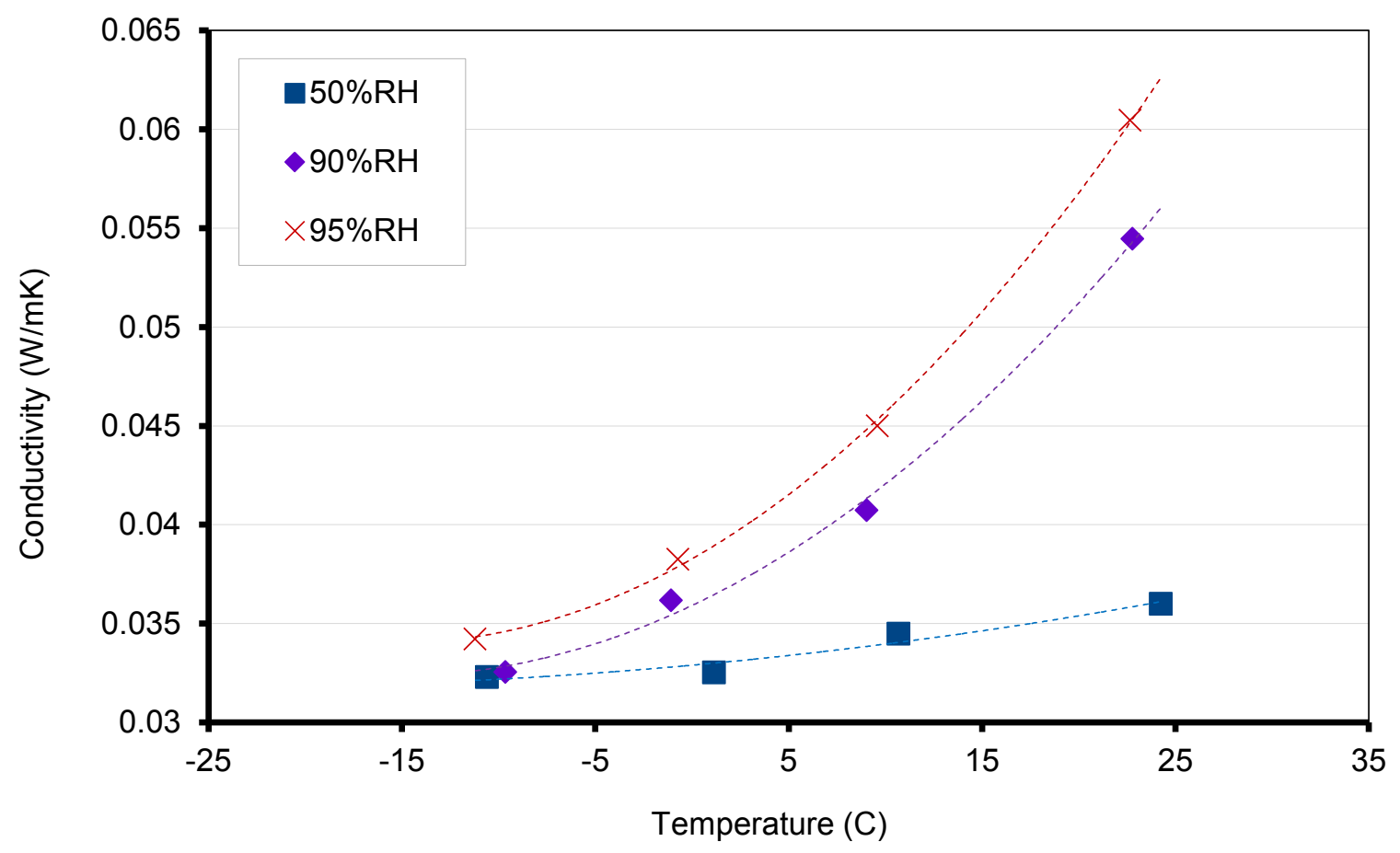

Figure 4.35: Effective Conductivity of Open Cell Polyurethane after 150 Freeze-Thaw Cycles and Moisture Exposure (PU-D).

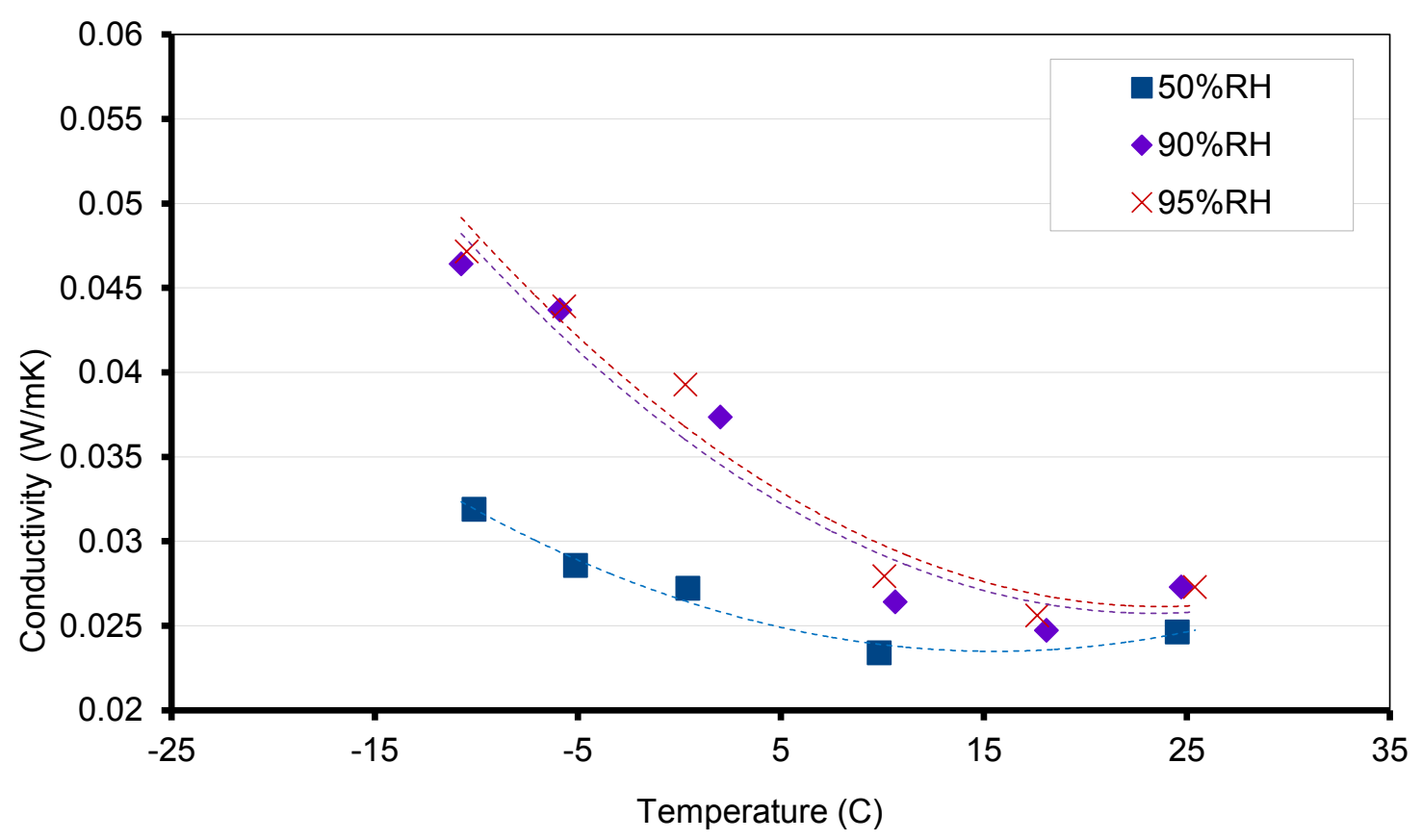

Figure 4.36: Effective Conductivity of Closed Cell Polyisocyanurate after 150 Freeze-Thaw Cycles and Moisture Exposure (PI-A). 


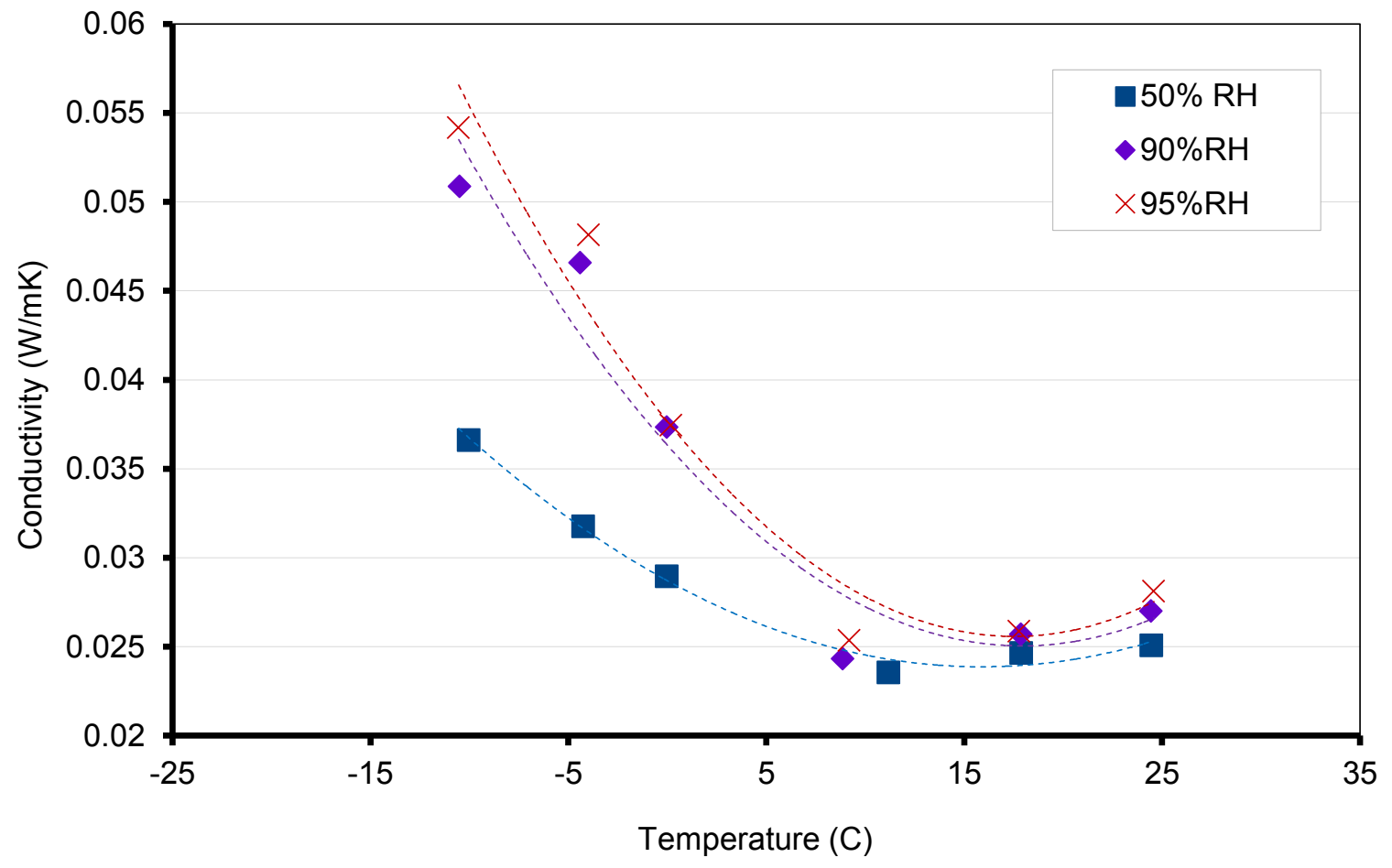

Figure 4.37: Effective Conductivity of Closed Cell Polyisocyanurate after 150 Freeze-Thaw Cycles and Moisture Exposure (PI-B). 


\section{Part 5: Discussion}

In this section, the results will be analyzed in further detail, discussing the potential causes of the results, as well as what they mean in the context of building design. Focus will be put on understanding the effects of elevated temperature aging and how it can be equated to real time, as well as visualizing effective conductivity as a function of both temperature and humidity. Additionally, wall assembly simulations using measured data for the materials will be described, and implications of effective conductivity before and after aging will be explored in a realistic simulated environment. 


\subsection{Aging Analysis}

As the aging process through elevated temperature occurred, the effects of increased conductivity at low temperatures caused by blowing agents appeared to increase in the polyisocyanurate material, and began to manifest in one closed cell polyurethane material (PU-A). Logic may suggest that as the blowing agent which is causing the issue escapes and is replaced with air, the effect of increased conductivity at low temperatures would actually be reduced in both the polyisocyanurate and polyurethane materials, this does not appear to be the case. This is likely due to the fact that, in the aging process the infiltration of outside air occurs at a faster rate than the blowing agent escapes (Shukla, 2017). As the thermal conductivity of outside air is higher than the conductivity of blowing agents, it causes the overall conductivity of the material to increase. Due to blowing agent remaining within the cells for a longer period of time, the negative effects at low temperatures remain.

A potential cause of the material PU-A experiencing this effect after aging tests occurred may be due to the infiltration of outside air mixing with the blowing agents, raising the average temperature at which the mixture begins to condense. Similarly, considering that the largest change in performance for the polyisocyanurate materials after elevated temperature aging was to the performance at low temperatures, it also suggests that as the blowing agents mix with outside air, the effects of condensation at low temperatures are further exaggerated.

The implication of temperature dependent conductivity and the effect that accelerated aging through elevated temperatures has on performance begins to show how single values for conductivity are untrustworthy in building design. Even when an advertised value takes into account aging through using LTTR testing methods, some foam materials, specifically polyisocyanurate materials have a much larger reduction in performance at cold temperatures experienced in a Canadian climate than at $24^{\circ} \mathrm{C}$. This means that even where LTTR is advertised, it may be understating the effects of aging on performance in the intended environment. 
While the results of the elevated temperature accelerated aging tests provide insight into the aging progression of the material, without understanding the rate of accelerated aging, the results are not as useful for understanding the aging progression in real time. In order to help equate the test method duration to the equivalent real time age, the Arrhenius equation (explained in methodology section 3.6) can aid in bridging the gap between elevated temperature accelerated aging and the equivalent time under realistic field or lab temperatures. Through one of the material manufacturers, it was found that the activation energy for most sprayed foam materials is around $58 \mathrm{KJ} / \mathrm{mol}$ which falls in line with general values reported by Kunic (2012). Using this value, a range of acceleration factors $\left(A F_{T}\right)$ for a range of use condition temperatures were determined based on the elevated temperature aging at $60^{\circ} \mathrm{C}$. Based on the result in Fig 5.1, it can be seen that when comparing 4 months of aging at $60^{\circ} \mathrm{C}$, the Arrhenius equation suggests that the equivalent time at $20^{\circ} \mathrm{C}$ would be 17.91 times longer (approximately 6 years). This result suggests that for the tested materials, the elevated temperature aging should provide a similar aging progression to materials left in a lab environment for 5 years as prescribed in LTTR test methods. Bridging the gap between lab storage and realistic use conditions becomes more difficult, due to the large difference in use temperatures that materials would be subjected to in Toronto, ON climate.

$$
A F_{T}=e^{-\frac{E_{A}}{K} \cdot\left(\frac{1}{T_{A}}-\frac{1}{T_{U}}\right)}
$$

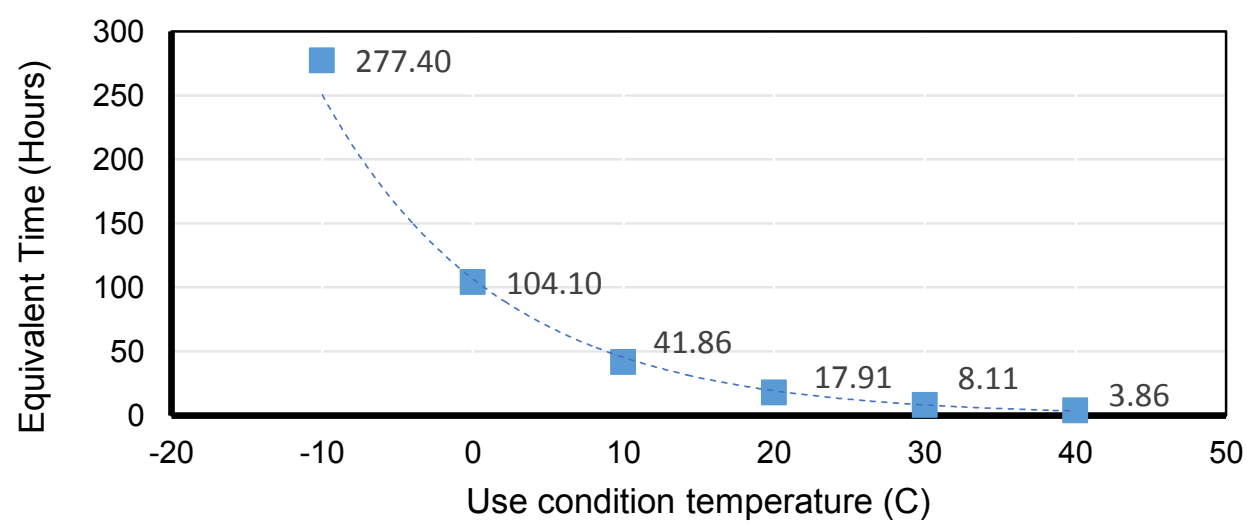

Figure 5.1: Acceleration Factors of foam materials for aging at $60^{\circ} \mathrm{C}$. 


\subsection{Hygrothermal Simulation Analysis}

In order to determine the effect of temperature dependent conductivity on the performance of a building envelope under realistic conditions, the WUFI hygrothermal analysis software was used to perform hygrothermal simulations on wall assemblies using measured values of the tested materials.

Material files based on the results of the material testing have been created. Each material incorporates the temperature and moisture dependent conductivity values found through measurements with the heat flow meter, as well as measured moisture storage function values, while other required material details were obtained through manufacturer technical data sheets. The simulations conditions were set in a Toronto, ON. climate, using the data file provided through the WUFI software.

These materials were applied to basic wood frame construction assemblies shown In Figs. 5.2 and 5.3. Figure 5.2 is a basic stud wall assembly. Figure 5.3 is the same assembly with an added layer of exterior insulation, typical of a wall renovation with the purpose of increasing the thermal resistance. Figure 5.4 presents a typical commercial roof assembly, which commonly make use of boards such as polyisocyanurate for insulation. In the simulations using Fig. 5.3, the investigated material was used as the exterior insulation layer only, in order to investigate the performance of each material with a mean temperature that is closer to the exterior environment. The simulations monitored the center as well as each face of the investigated insulation layer, and outputs of the temperatures over the course of January and July at these locations in the assembly were obtained. These temperatures were used in combination with the temperature dependent conductivity curves developed through lab measurements before and after accelerated aging, in order to create a visualization of the dynamic effective conductivity over the duration of the simulation. These graphs reveal that when taking into account effective conductivity and aging, the thermal performance of a material varies based on the environmental conditions and the location within the assembly design. 


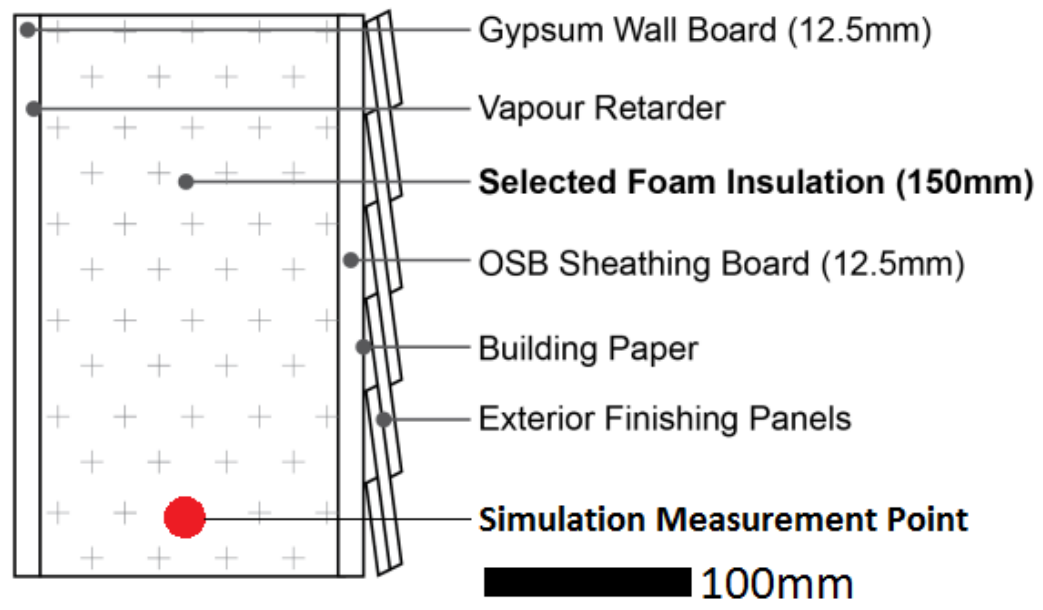

Figure 5.2: Standard Wood Frame Wall Assembly.

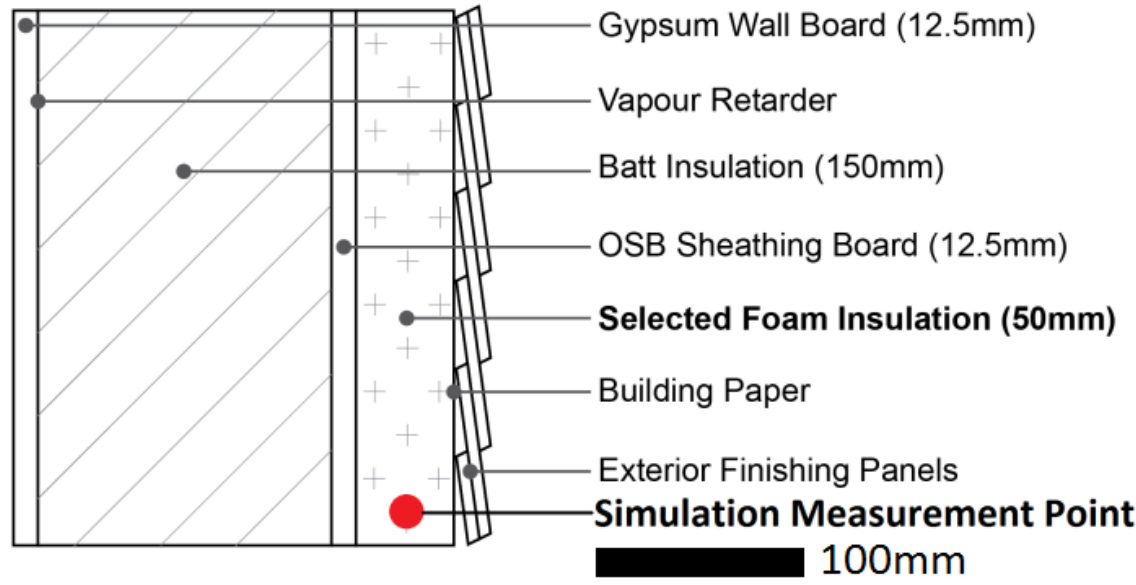

Figure 5.3: Exterior Insulated Wall Assembly.

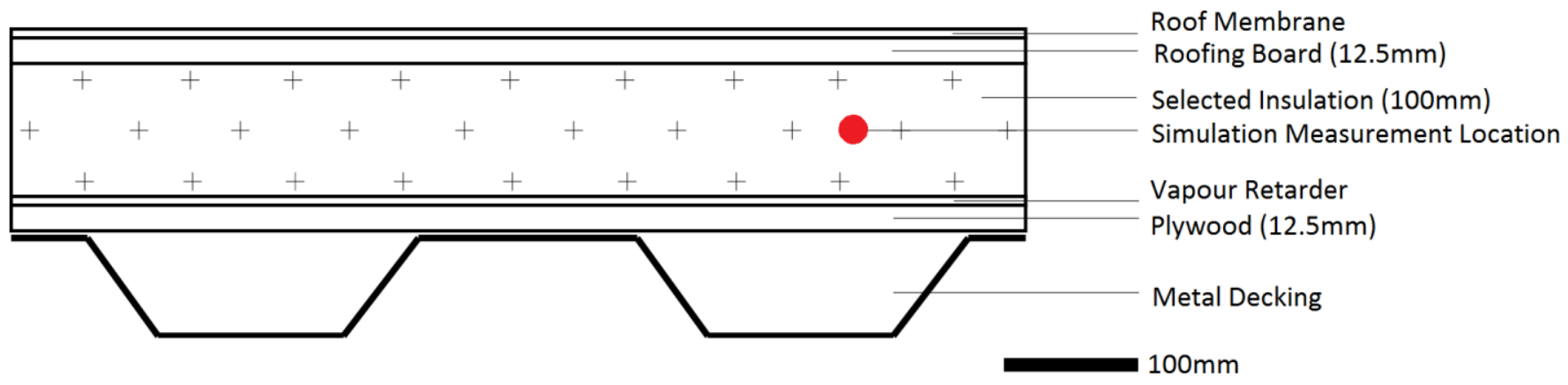

Figure 5.4: Flat Commercial Roof Assembly. 
For the following simulation results, temperature and humidity conditions were measured in the center point of the insulation layer, as well as each face of the insulation layer. The most accurate determination of effective conductivity would consider the full spectrum of temperature and moisture, as presented in section 5.1 in 3-D surface graphs. The conductivities in the following graphs were calculated using the measured temperature dependent conductivity curves measured in result section 4.2 elevated temperature accelerated aging. This limitation means that the extent at which materials are affected by high moisture levels are unclear in this portion of analysis. However, these results still provide a clear indication of the variation in effective conductivity for materials before and after accelerated aging through elevated temperature. Simulation analysis considering both temperature and humidity in combination to calculate the effective conductivity of the material is currently in development for future work. It is important to note that while the graphical analysis of the simulation outputs is currently unable to account for moisture level in calculating the effective conductivity, the simulation itself does consider both temperature and humidity dependent conductivity through an iterative process based on the input values for each material. These specific values were taken from the measured temperature and moisture dependent conductivity curves, and can be found in appendix section 9.1 .

In the initial simulations, in order to observe the effect of the insulations dynamic conductivity with the most clarity, a basic wood stud wall assembly with OSB and gypsum board on either side will be simulated filled with each insulating material. This type of wall assembly will achieve nearly all of its effective U-value through the insulation alone. The following charts show the average effective conductivity during each month of the simulation. Using these graphs, it can be determined how each material performs on average over a period of time. It is important to understand these values because in a cold Canadian climate, the performance of the insulating material is integral to the performance of the building as a whole, especially in winter months. 

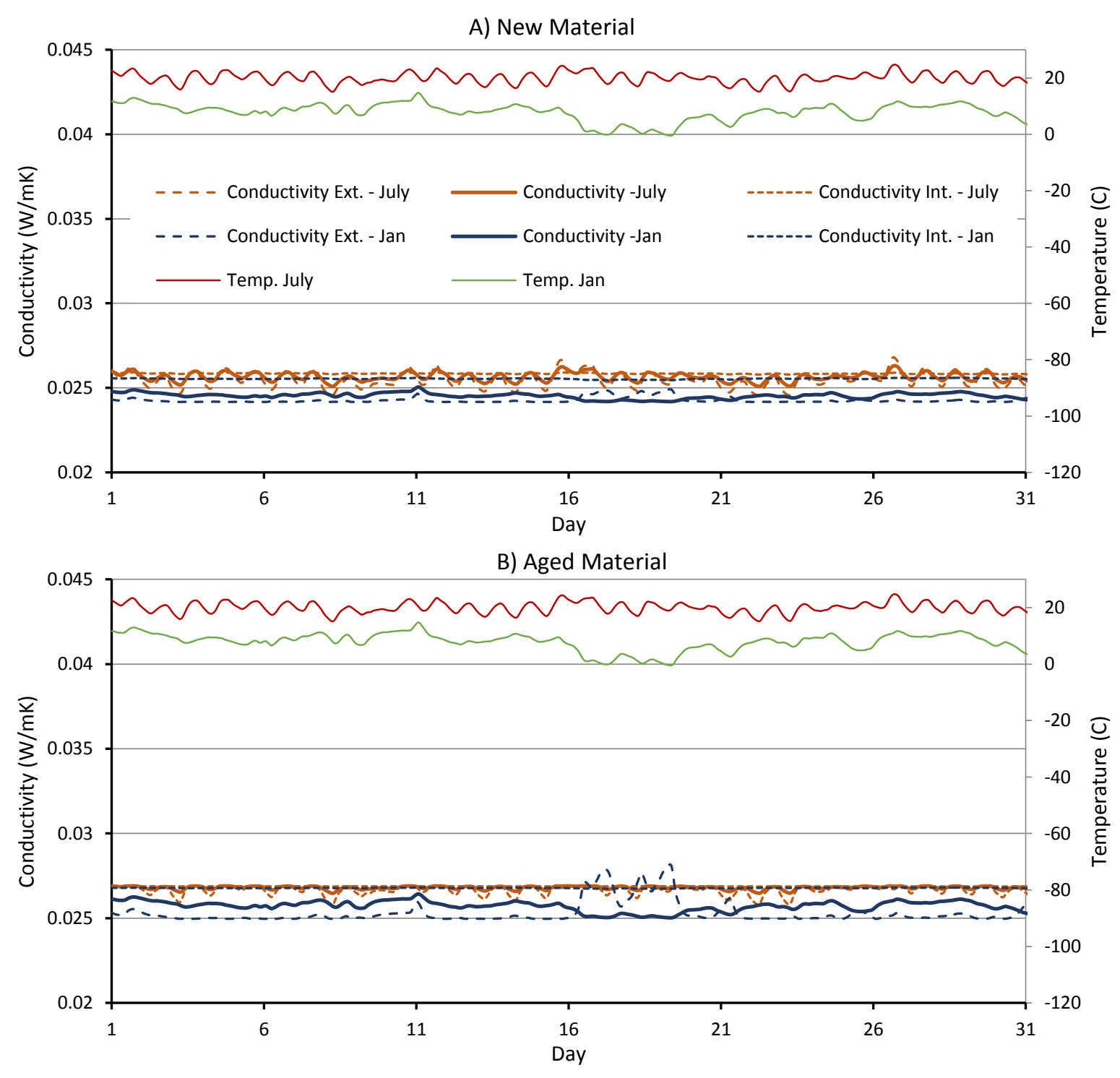

Figure 5.5: Effective Conductivity for Interior Insulation Layer of Material PU-A.

The graph in Fig.5.5a displays the effective thermal conductivity of material PU-A within the wall assembly of Fig.5.2. The solid conductivity line presents the measurement at the center point of the insulation layer, while the dashed lines above and below present the measurement at the inner and outer face of the material. It can be seen that towards the inside face of the insulation, the conductivity remains very stable, as the temperature at this location is closer to the steady interior temperature than the exterior environment. Towards the exterior surface of the material, it can be seen that the material performance becomes less consistent as the temperature fluctuates. Over the course of both January and 
July, the material performance remains relatively consistent as it would be expected based on the unaged measurements of the material shown in Fig. 4.7 (pg.44). In Fig. 5.5b, the same wall assembly was simulated using the final aged values of PU-A. It can be seen that the effective conductivity remains consistent, though performs overall slightly worse, as would be expected by the aging results in section 4.2 .
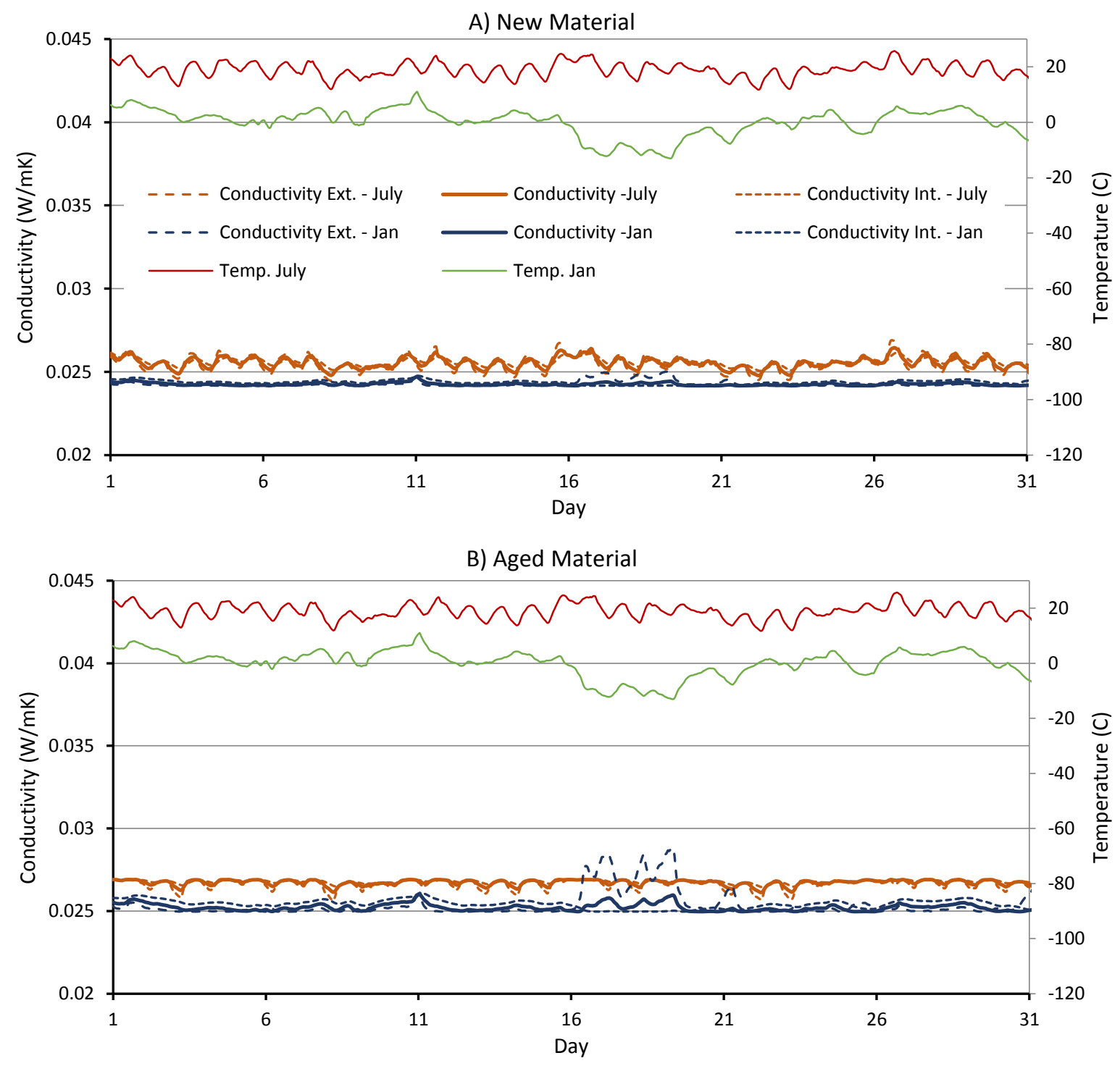

Figure 5.6: Effective Conductivity for Exterior Insulation Layer of Material PU-A.

In Fig. 5.6a, the material PU-A was simulated within the wall assembly of Fig. 5.3. The implication of monitoring this material when used as an exterior insulation layer is that the temperature within the material is closer to the exterior temperature. In Fig. 5.6b, this exterior insulation simulation was 
repeated using values of the final aged PU-A material. In Fig. 5.6, the results resemble what was found in Fig 5.5. Similarly, when implemented into a roof assembly (Fig 5.7), material PU-A remains relatively consistent in performance over the course of January and July. Due to the relatively stable conductivity of PU-A over a range of temperatures, the performance is consistent wherever the material is used within the wall assembly. Across all three assemblies, the most impactful effect is the increase in conductivity due to aging effects. The effective conductivity of PU-A was calculated to be consistently $7-10 \%$ higher after aging based on simulations using measured data.
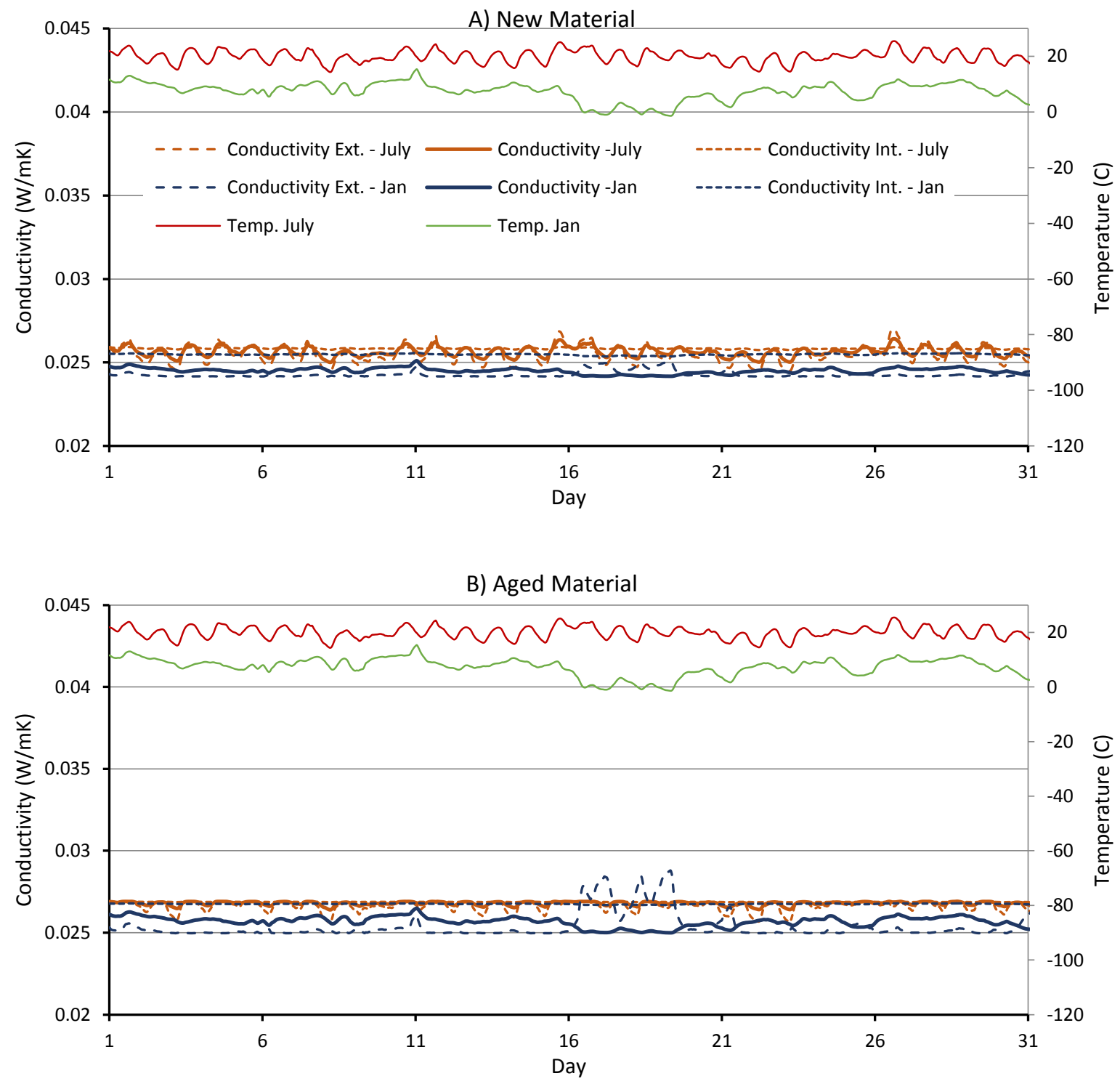

Figure 5.7: Effective Conductivity for Roof Insulation Layer of Material PU-A 
Similarly, PU-D has a relatively consistent effective conductivity as open cell polyurethane. (Fig 5.85.10) The range of performance within the material is slightly larger than PU-A in the winter months. This is due to the linear temperature dependent conductivity the foam has, reducing at cold temperatures, while the temperature dependent conductivity in PU-A plateaued and showed signs of increasing at low temperatures. In the case of PU-D, the conductivity is calculated to be decreasing towards the exterior surface in January where the temperature is low.
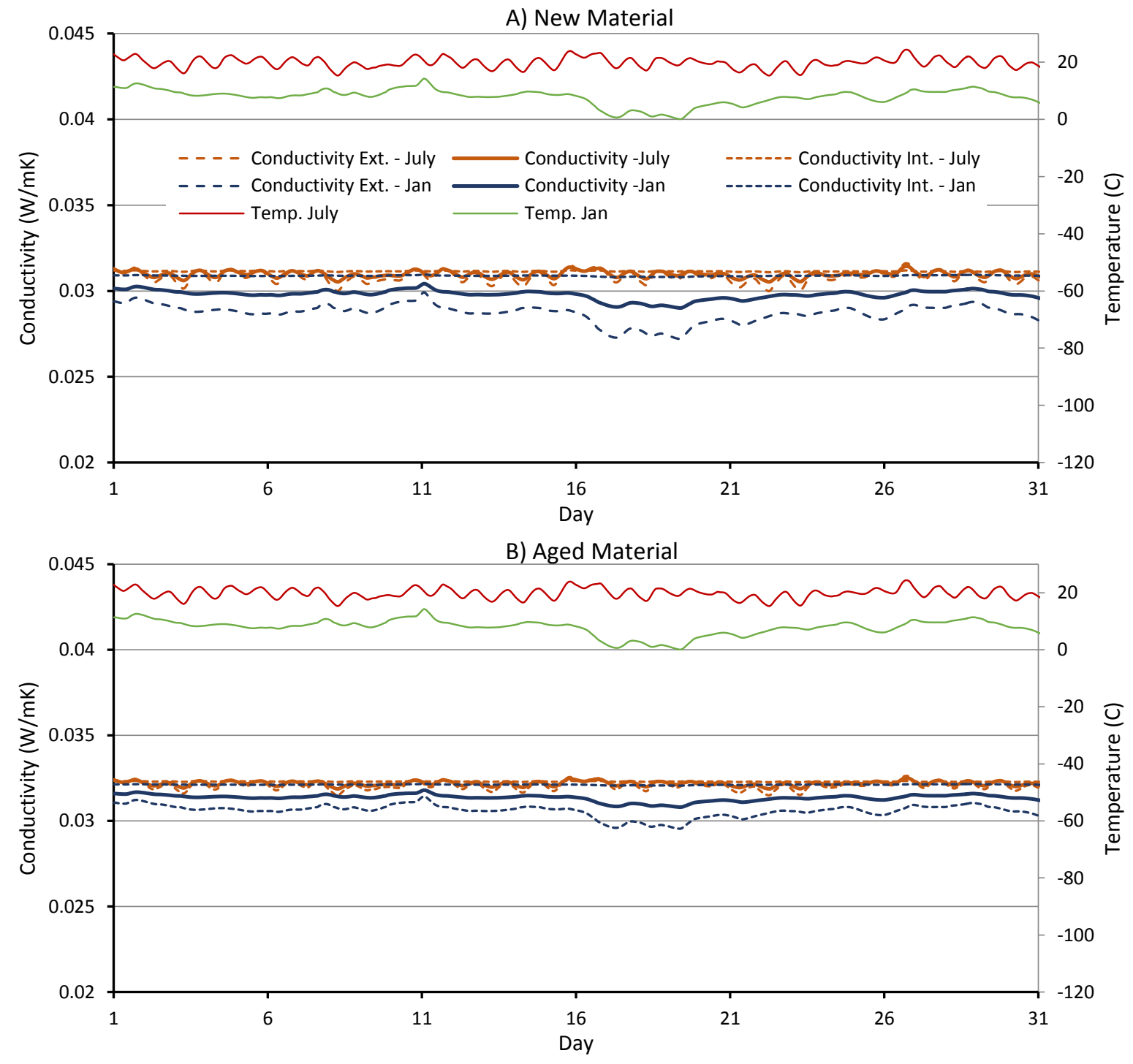

Figure 5.8: Effective Conductivity for Interior Insulation Layer of Material PU-D 


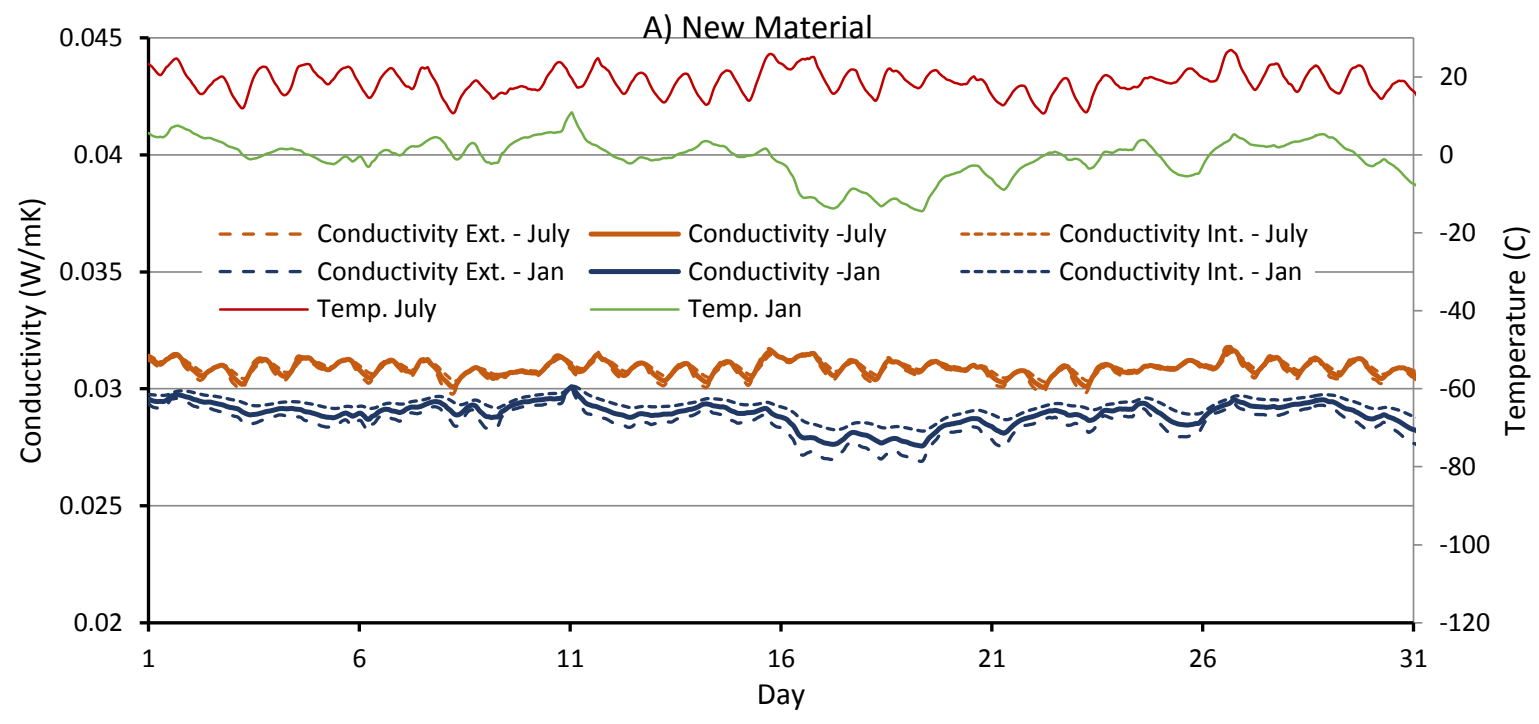

B) Aged Material

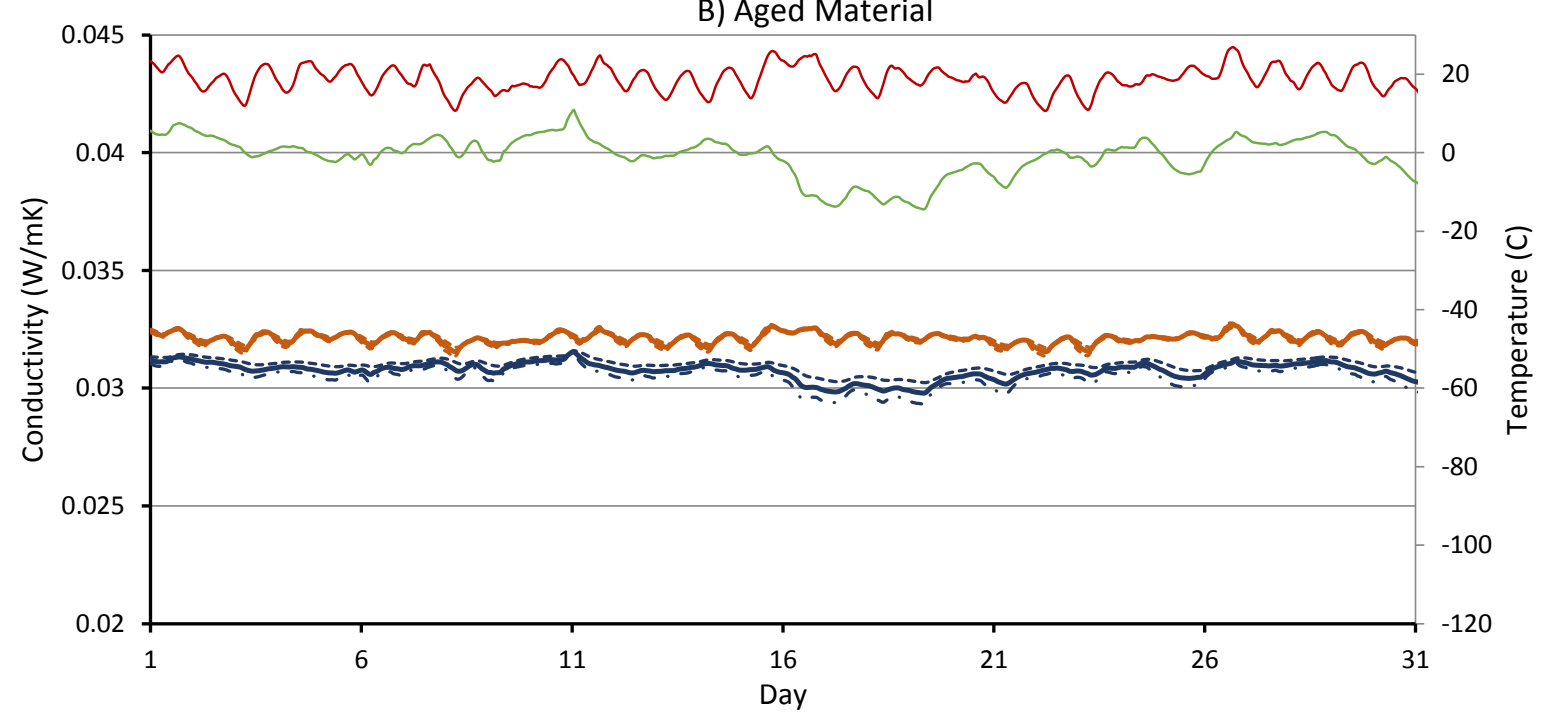

Figure 5.9: Effective Conductivity for Exterior Insulation Layer of Material PU-D

In the simulation using an exterior insulation layer (Fig 5.9), the temperatures within the material are closer to the exterior temperatures, also with a decreased range in temperatures across the layer. This causes the material to perform more consistently across the entire layer, with minimal difference between the performance at the interior and exterior surfaces of the material at any given time. In this simulation it can be seen that the open cell foam performs better in the winter at low temperatures, as suggested by its temperature dependent conductivity curve. In Fig 5.10, the result of PU-D used as a roof insulation layer can be seen. Similarly to the previous results, the material performs slightly better 
towards the colder surface of the insulation. An important note regarding the calculated effective conductivity of PU-D is that while the material performs fairly consistently over a range of temperatures, changing moisture levels within the material will have a larger impact on the material performance. In situations where the interior of the wall becomes wet, or insulation somehow becomes exposed to very high moisture levels, the open cell materials, will take the biggest hit to performance based on the results in section 4.3 (pg. 51).
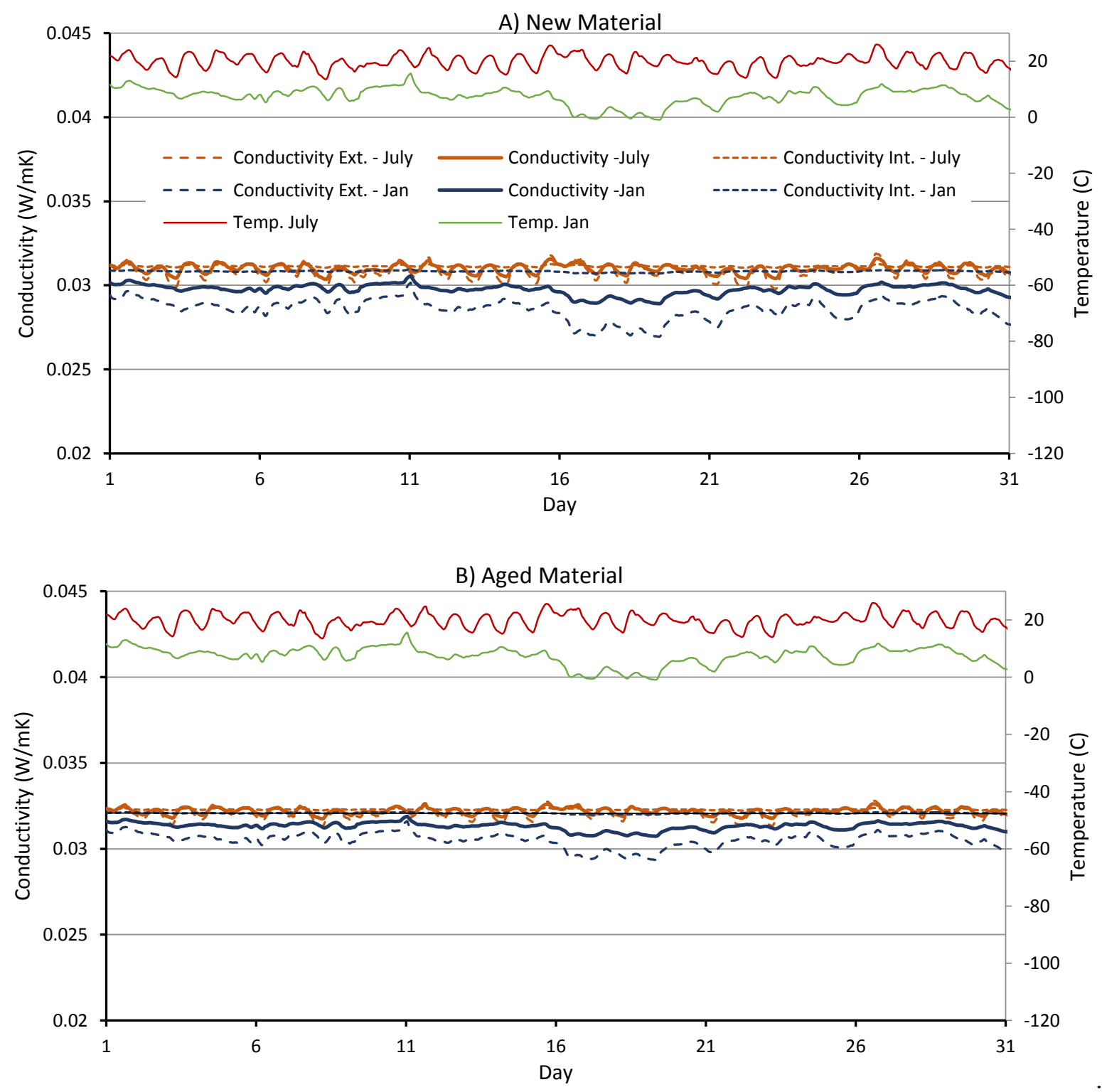

Figure 5.10: Effective Conductivity for Roof Insulation Layer of Material PU-D 

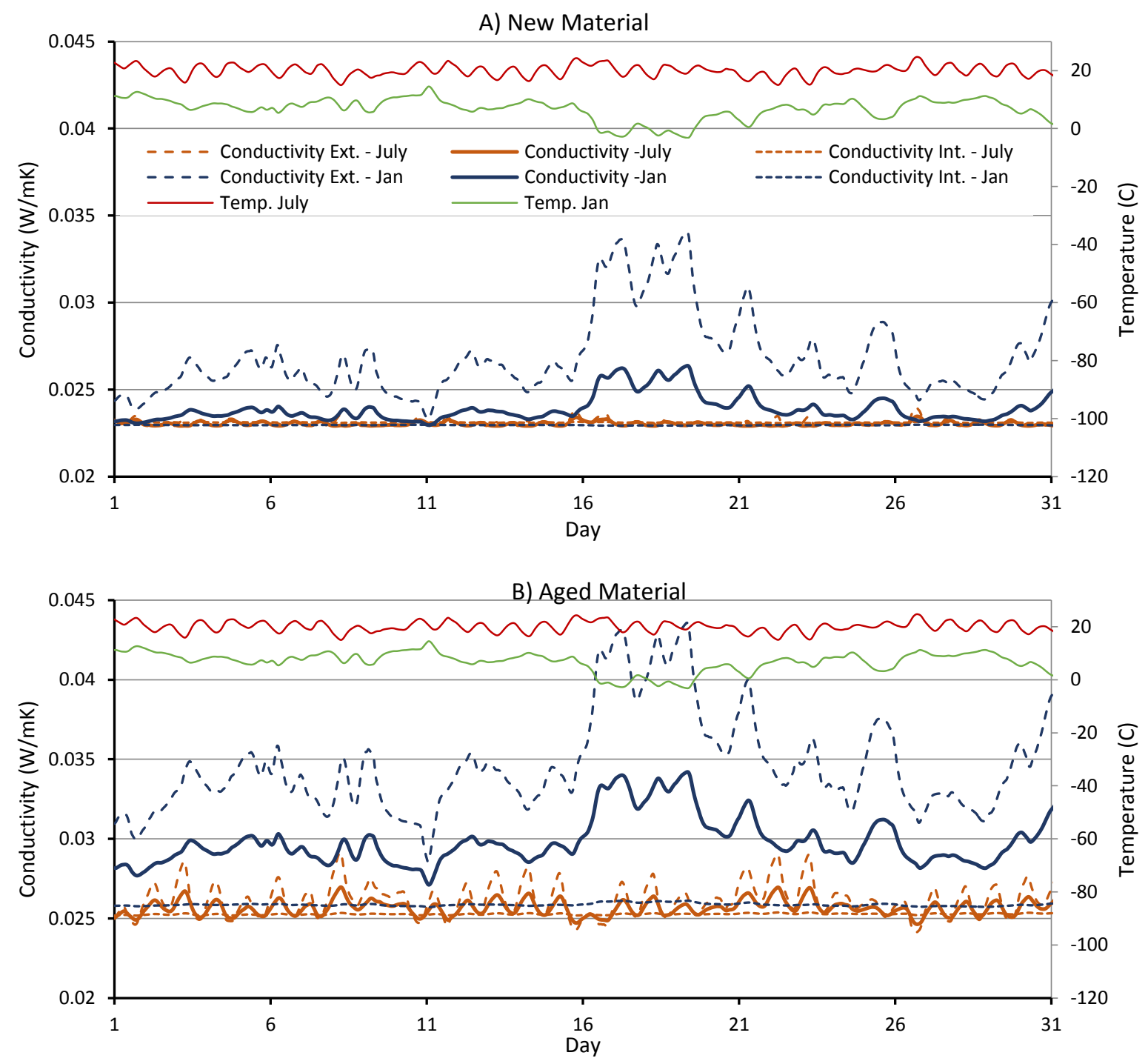

Figure 5.11: Effective Conductivity for Interior Insulation Layer of Material PI-B.

In contrast to the results of PU-A and PU-D, the polyisocyanurate material PI-B shows a very inconsistent temperature dependent conductivity, when simulated using the wall assembly in Fig 5.2. It experiences major increases as the temperature is reduced and after aging. In Fig. 5.11, simulations using unaged and aged PI-B materials, reveal that the effective conductivity are less consistent during the coldest periods of January in both simulations, especially after aging (Fig. 5.11b). At the center of the insulation layer, the conductivity performs $\sim 20 \%$ worse than in July after aging. Towards the colder outside surface of the material in January indicated by the uppermost dashed line in Fig 5.11, the effective conductivity sees increases of up to $70 \%$ higher than the conductivity in July. 
In the following scenarios, the wall assembly in Fig. 5.3 will be used. Through the temperature plots measured at the insulation center point from the simulations, it was made clear that as the outside temperature drops below $-20^{\circ} \mathrm{C}$, the mean temperature within the insulation is just beginning to drop below $0^{\circ} \mathrm{C}$. In the next simulations, it will be shown that as a secondary insulation layer applied to the exterior of an existing wood frame wall filled with batt insulation, the mean temperature within the foam layer will be closer to the exterior temperature than in the previous simulation, and the negative effects of increasing conductivities at low temperatures in the polyisocyanurate materials will be more pronounced.
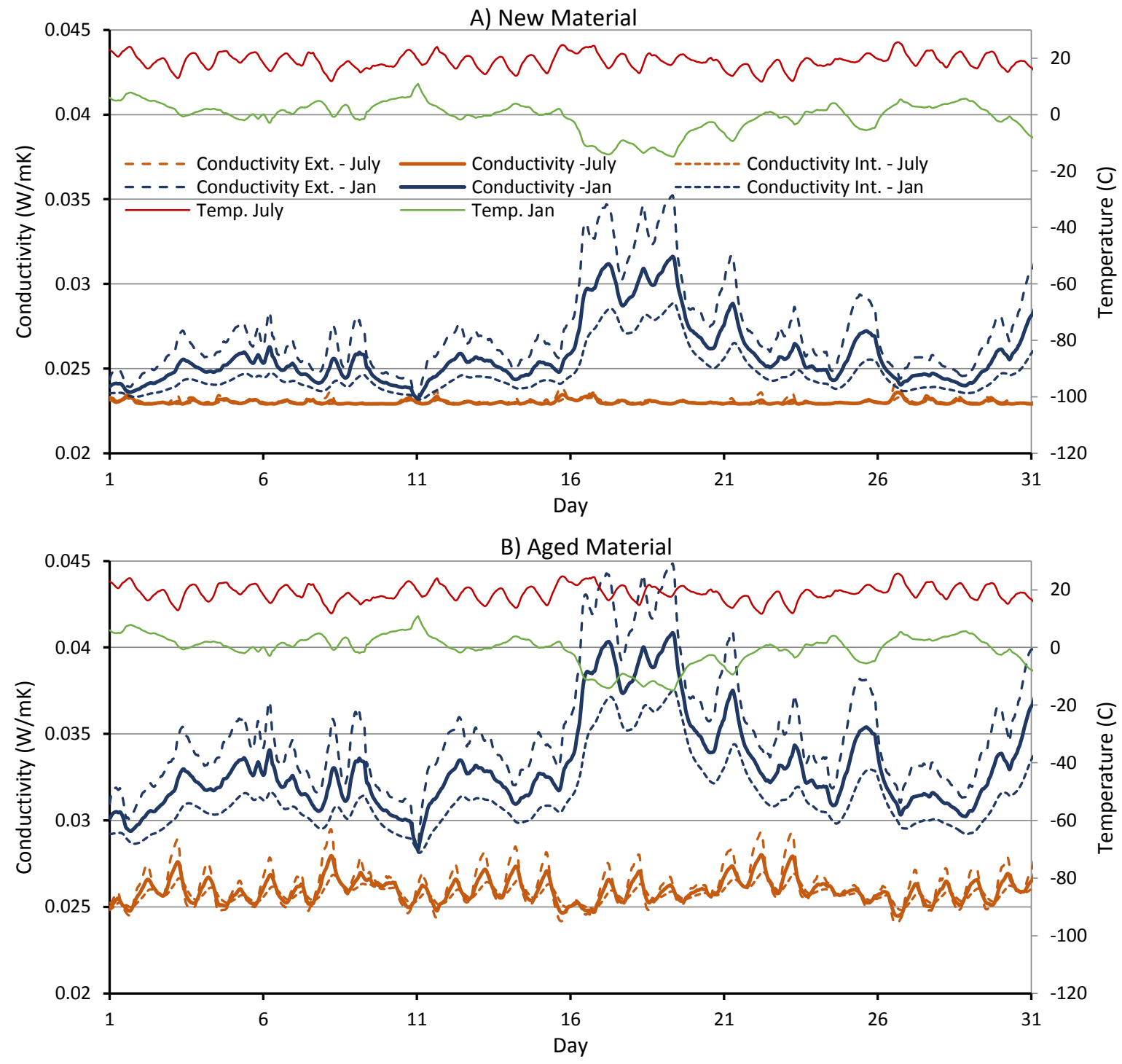

Figure 5.12: Effective Conductivity for Exterior Insulation Layer of Material PI-B. 
When these simulations were repeated with the insulating material used as the exterior layer, the effective conductivity becomes even more variable over a range of temperatures. In Fig. 5.12, it can be seen that even before aging the PI-B material has massive swings in effective conductivity when used as an exterior insulation layer in the winter. After aging (Fig. 12b), this effect becomes much worse, with nearly $40 \%$ reduction in performance during the most of January, at times the conductivity increasing up to $80 \%$ from July, with significant inconsistency during July as well. It is clear that when used as an exterior insulation layer, even the interior surface of the insulation will still be affected by the exterior temperature further reducing the overall effectiveness of the insulation layer.
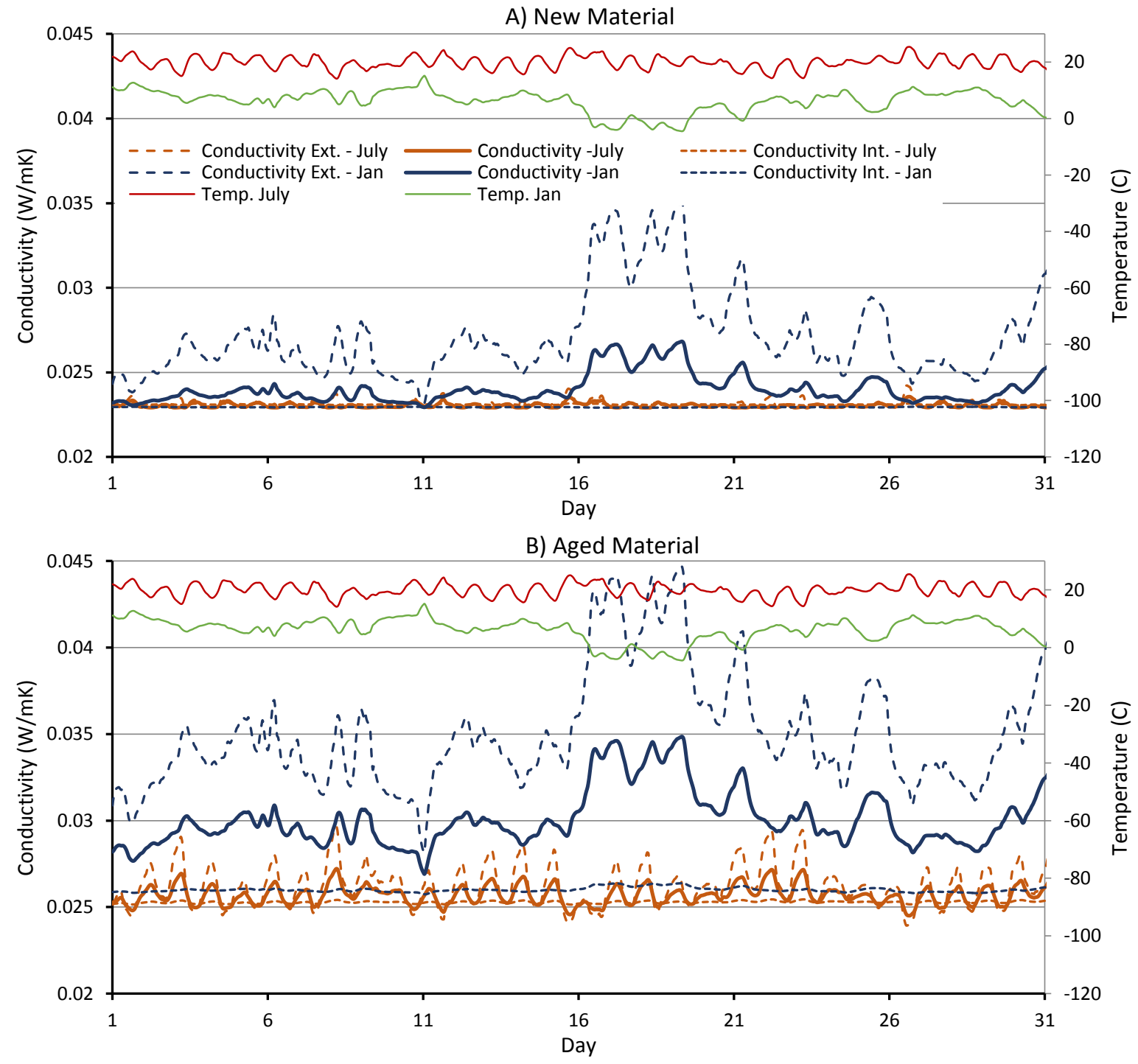

Figure 5.13: Effective Conductivity for Roof Insulation Layer of Material PI-B. 
In Fig. 5.13, the commercial roof assembly (Fig 5.4) was investigated using measured material data for PI-B. These results reveal similar results to the basic wall assembly with a single insulation layer. The polyisocyanurate material experiences spikes in conductivity during the coldest parts of the year, especially towards the exterior surface in the winter. Similar, to the other simulations, the effects are further exaggerated after aging.

Through these results, it is clear that the method in which a wall assembly is designed will have a great impact on the performance of the materials themselves due to the temperature gradient through the wall assembly, increasing the importance of position within the assembly for certain types of material. From these simulations, it can be concluded that when the temperature-dependent conductivity of a material is known, those materials such as the polyurethane that has an reduced or consistent conductivity at cold temperatures would make a better performing exterior insulation layer in a cold environment, while the materials that have higher conductivities at lower temperatures such as the polyisocyanurate, should potentially be avoided or designed to accommodate the weakness in cold operative conditions. Additionally, these simulations help visualize the difference in performance over the course of a building life cycle. As materials age, thermal properties change as well. In the case of these investigated materials, after aging, it can be seen that in the polyurethane materials, while the average conductivity increases, the effective conductivity remains relatively consistent, while for polyisocyanurate materials, the effective conductivity range becomes much larger. With a larger effective conductivity range, calculating the actual performance of a certain material may become troublesome in practice, due to the constantly changing environmental conditions that will have major impacts on the material performance.

These simulations provide insight into how even small changes in the effective conductivity of an insulating material due to varying environmental conditions or aging can have major effects on the performance of a building envelope. In situations where high degree of accuracy is critical, such as a 
passive house building design, understanding the effective conductivity of materials is very important. In these types of high performance buildings, calculations made during the design phase, play a large role in determining energy loads and equipment choices. If the input data for material conductivity is inaccurate, it could lead to issues during the life-cycle of the building, when the envelope performs at a lower level in certain weather conditions or within the first few years after construction due to the early stages of aging. Understanding the effect of temperature-dependent conductivity will be very beneficial to architects and engineers in the design of passive houses and other high-efficiency houses. If potential issues with effective conductivity are identified in the early stages of the project, the design can be altered to compensate where necessary, or material choices can be reconsidered, leading to a reduced chance for unexpected performance issues in a built envelope. 


\subsection{Effective Conductivity Surface Meshes}

In Figs. 5.14-5.19, 3-axis graphics developed from the measured data in result section 4.3, clearly display the phenomenon of changing effective conductivity over a range of temperature and humidity conditions. This type of representation will help bridge the gap between the practice of inaccurately calculating the performance of building envelopes through using a static material conductivity and the understanding that material conductivity is a function of the environmental conditions, and changes dynamically for temperatures and moisture levels. Through looking at portions of the 3-D surfaces, it becomes easy to visualize the effective conductivity of the materials in a given climate, allowing designers to more easily see under which conditions certain materials should be avoided. These figures present mesh surfaces which connect each measured point. If a material conductivity was assumed to be a single value, it would only represent a single point on these surfaces.

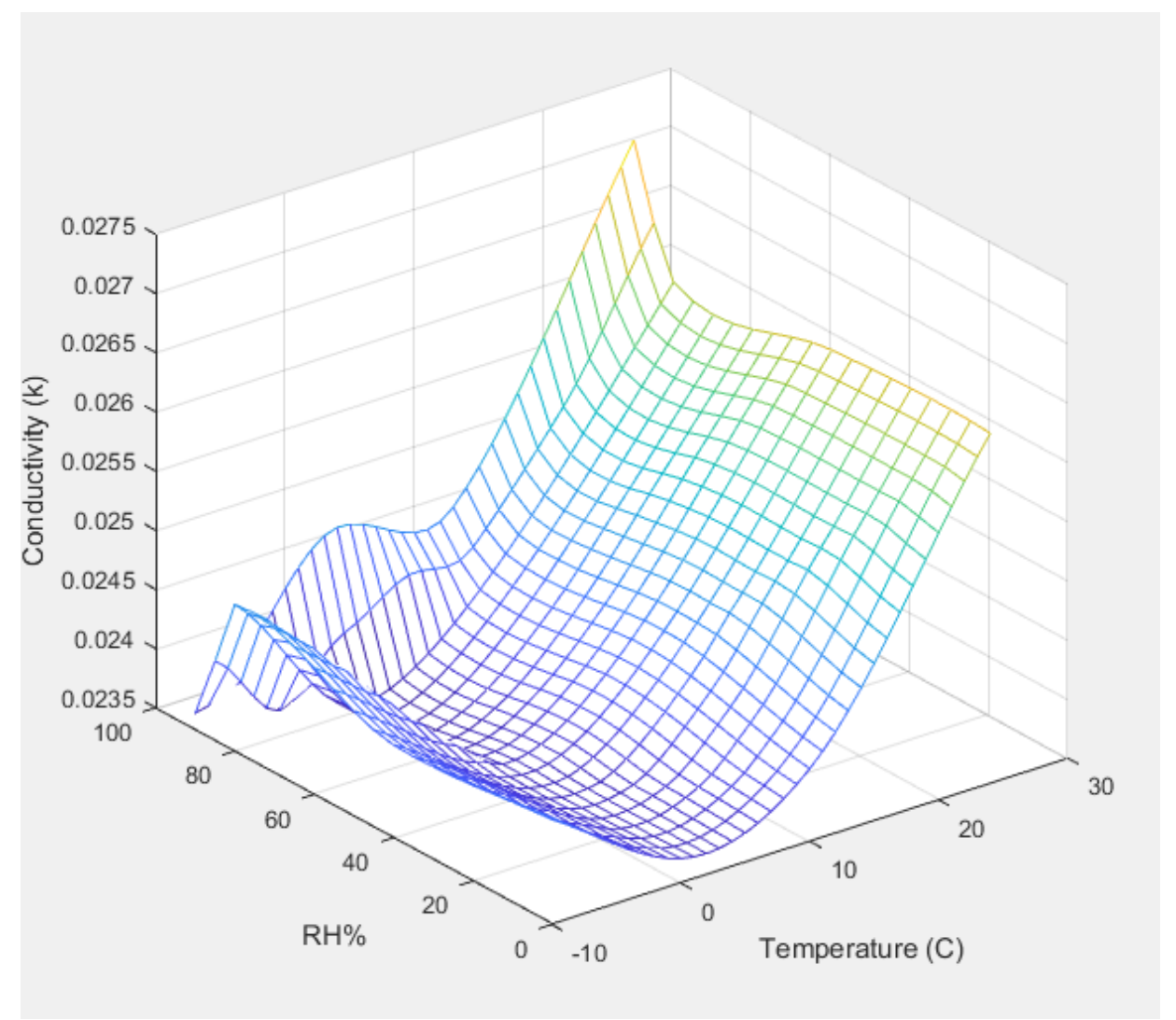

Figure 5.14: Effective Conductivity $(\mathrm{W} / \mathrm{m} * \mathrm{~K})$ of Closed Cell Polyurethane across a range of moisture levels and temperatures $(P U-A)$. 


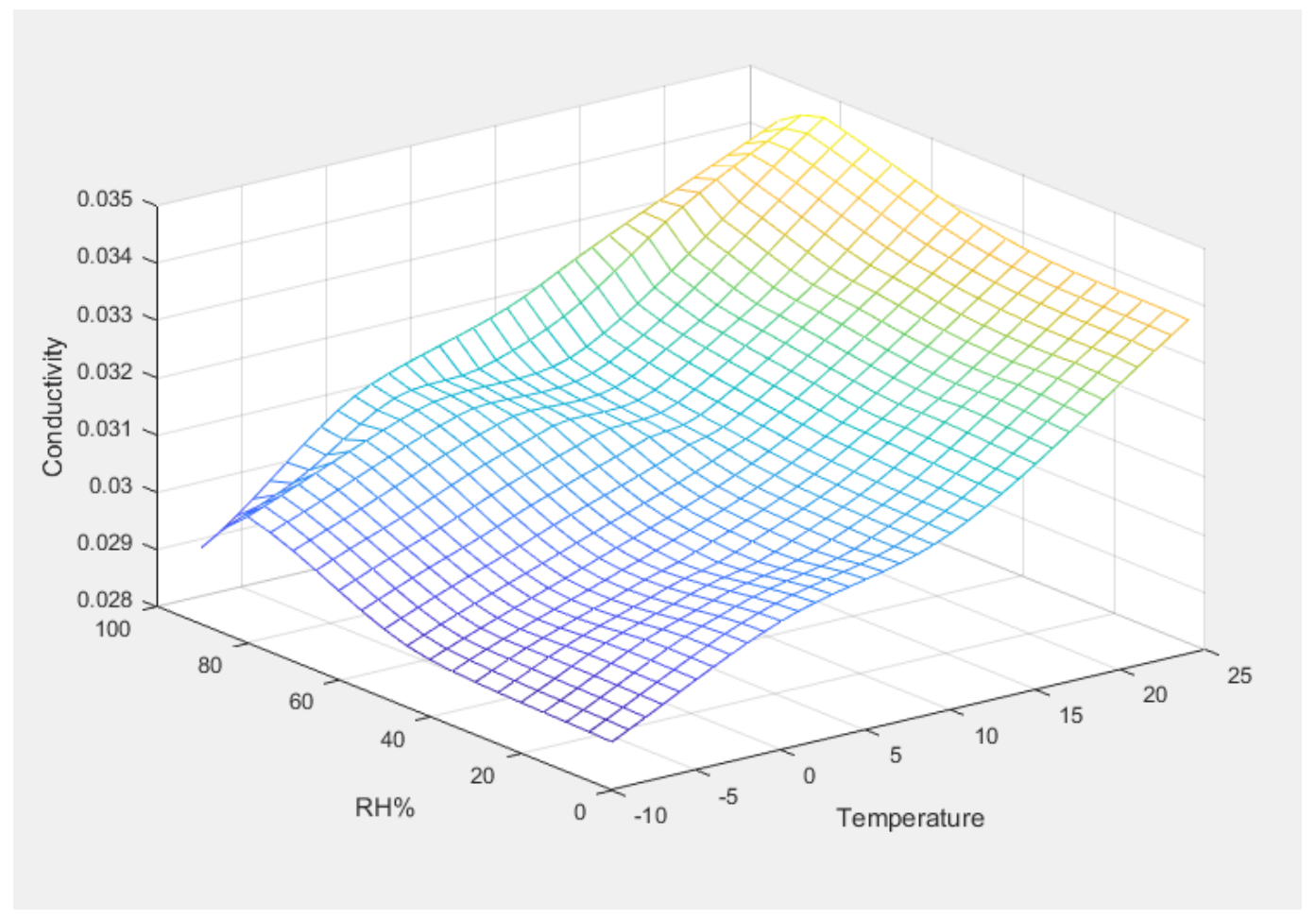

Figure 5.15: Effective Conductivity $\left(\mathrm{W} / \mathrm{m}^{*} \mathrm{~K}\right)$ of Closed Cell Polyurethane across a range of moisture levels and temperatures $(P U-B)$.

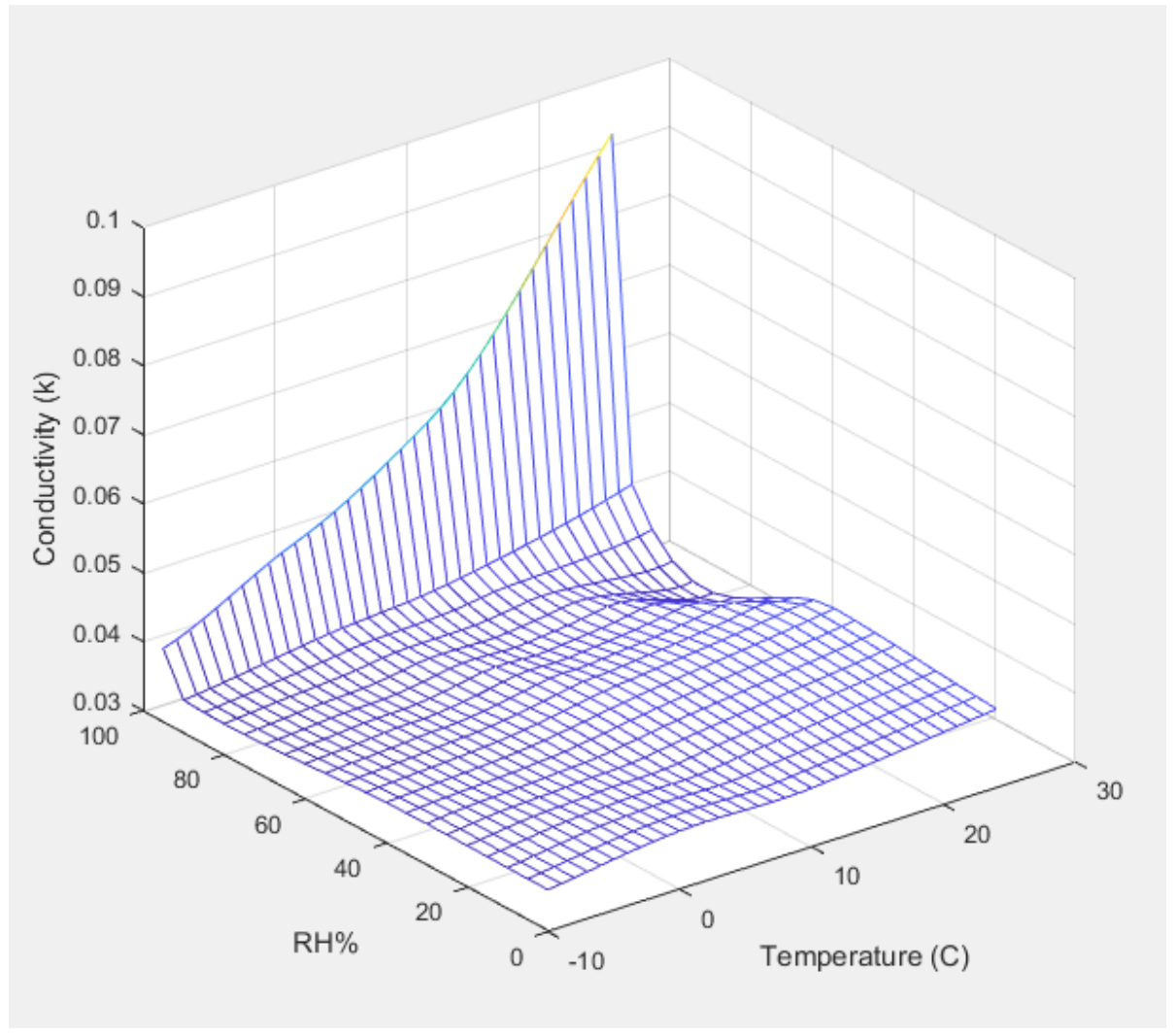

Figure 5.16: Effective Conductivity $\left(\mathrm{W} / \mathrm{m}^{*} \mathrm{~K}\right)$ of Open Cell Polyurethane across a range of moisture levels and temperatures $(P U-C)$. 


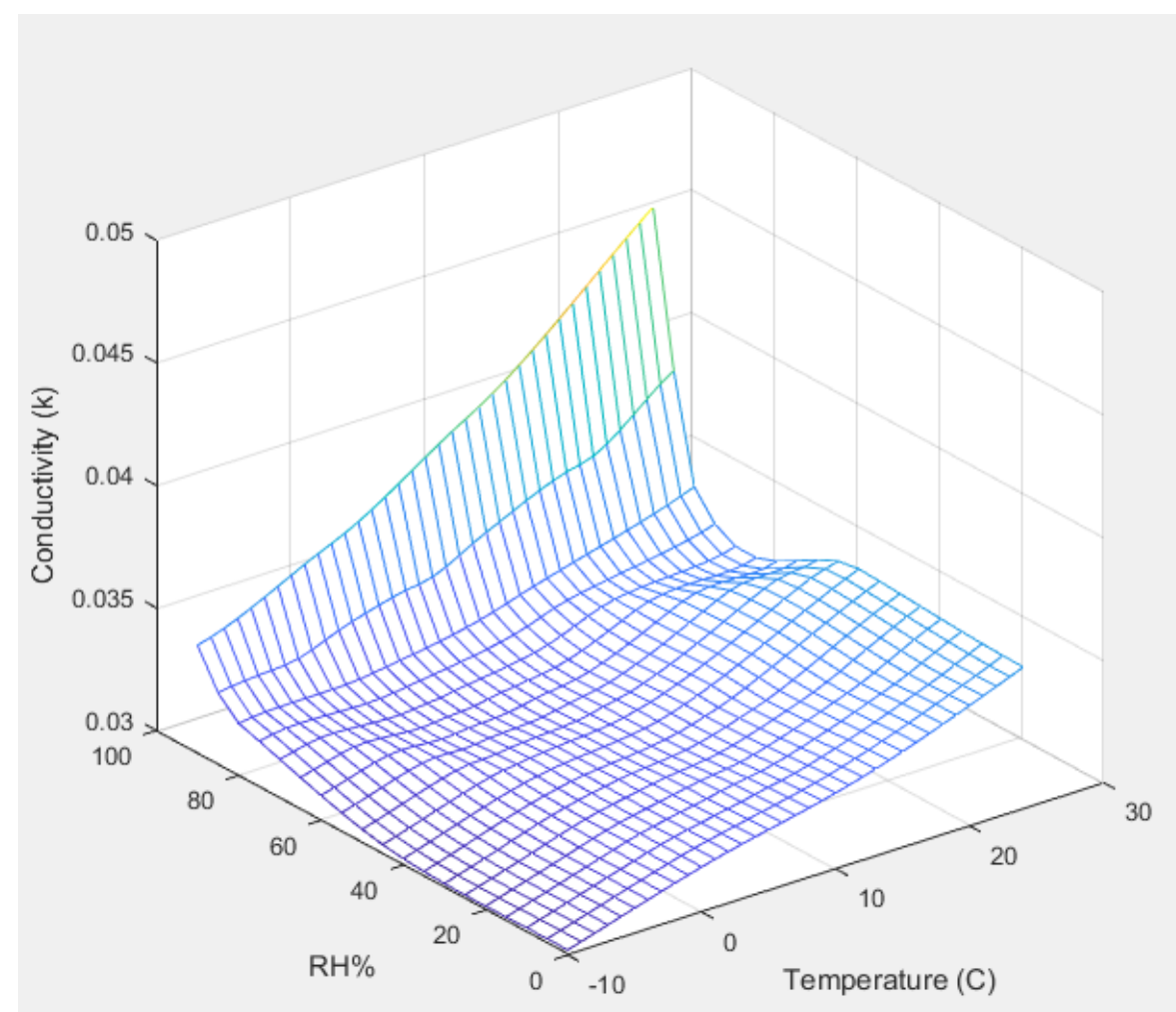

Figure 5.17: Effective Conductivity $\left(\mathrm{W} / \mathrm{m}^{*} \mathrm{~K}\right)$ of Open Cell Polyurethane across a range of moisture levels and temperatures $(P U-D)$.

Through these graphics, it can be seen that in the three types of materials being explored, different relationships exist. In the closed cell polyurethane foams (Fig. 5.2-5.3), we see that the conductivity is relatively consistent across humidity levels until it surpasses the $95 \%$ range, as well as across the range of temperatures. It is important to note that for both closed cell polyurethane material graphs, the z-scale is quite small, allowing the details of the graph to be seen. While the shape of the graphs look comparably inconsistent to the following figures, the range of effective conductivity is relatively quite small. The conductivity only slightly increases as both temperature and humidity increase, and sees a slight increase in conductivity at very low temperatures in PU-A. An important note for these materials is that even after the effects of environmental conditions, the changes in conductivity are relatively small compared with polyisocyanurate materials. The open cell polyurethane materials (Fig. 5.4-5.5) have a similar relationship with environmental conditions to the closed cell materials, with the largest difference being the extent at which high moisture levels increase the 
conductivity. In the open cell material, the effect of high moisture dwarfs the effect of changing temperature. This is expected due to the sponge like nature of the open cell foams providing a very high moisture storage capacity. In Fig 5.18-5.19, the material conductivity slightly increases with higher humidity, but also rapidly increases as the temperature decreases to lower values in both polyisocyanurates. In situations where high humidity and low temperatures are combined, these effects combined push the conductivity values very high.

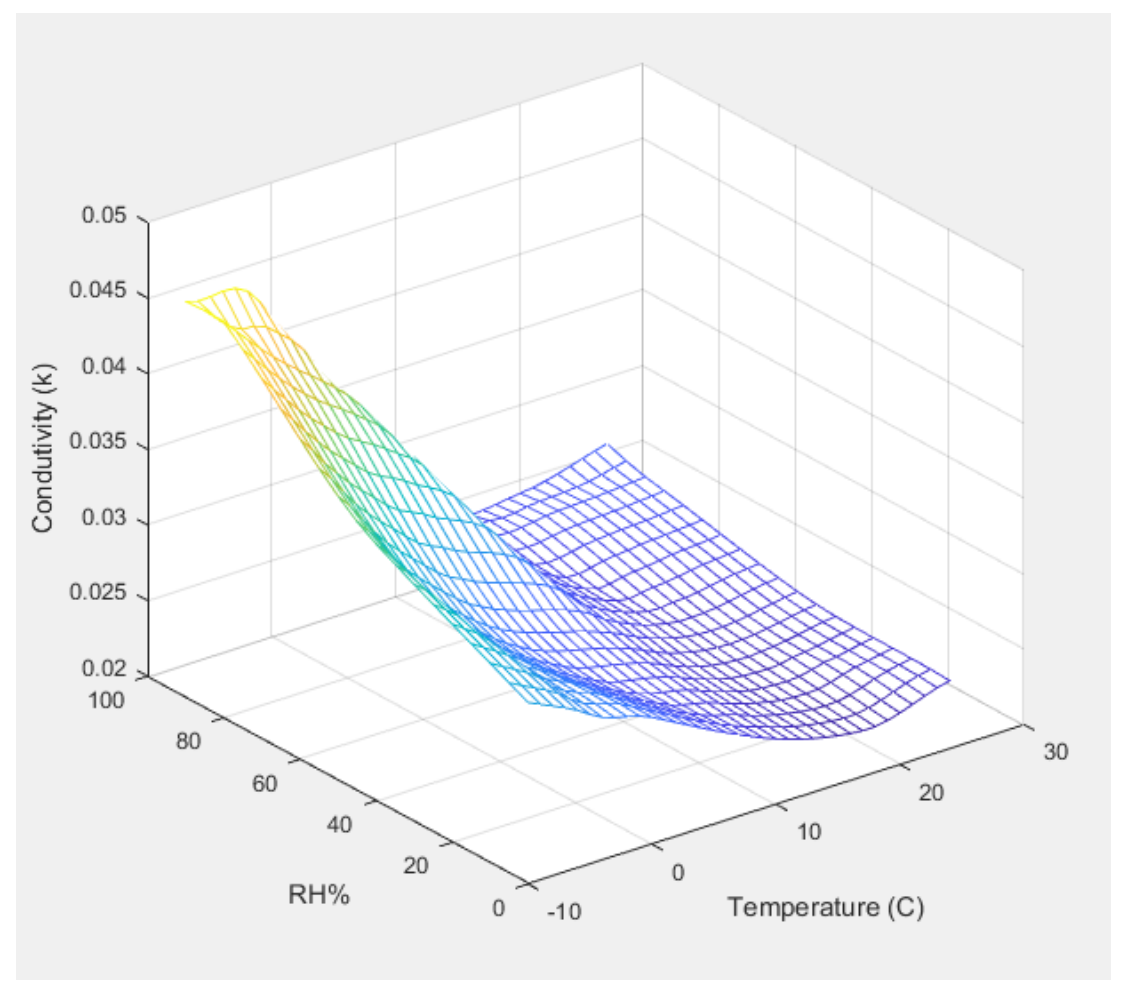

Figure 5.18: Effective Conductivity $(\mathrm{W} / \mathrm{m} * \mathrm{~K})$ of Closed Cell Polyisocyanurate across a range of moisture levels and temperatures (PI-A). 


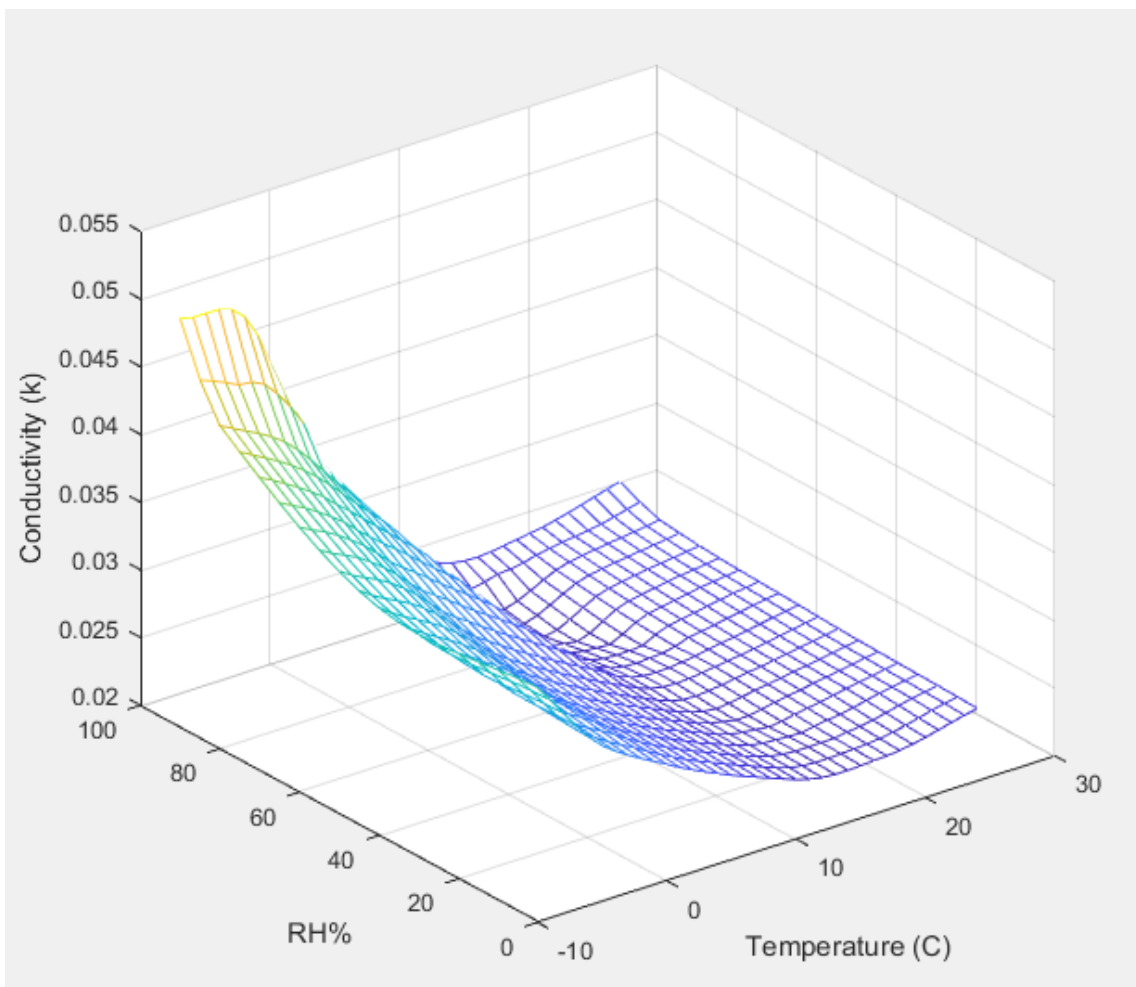

Figure 5.19: Effective Conductivity $(\mathrm{W} / \mathrm{m} * \mathrm{~K})$ of Closed Cell Polyisocyanurate across a range of moisture levels and temperatures (PI-B).

The graphics presented for these materials reveal that while similar materials may experience similar trends, each material performs differently under varying conditions. This emphasizes the importance in understanding each specific material being used in a building design. It is important to note that for these mesh surfaces, the Z-axis scale was selected in order to more closely assess the shapes of the surfaces, meaning that the scale between material graphs may not be the same and should be noted closely.

General assumptions and singular values will be very misleading in determining the actual effective conductivity of materials without specific laboratory measured data. Due to the way these mesh surfaces were developed, while they are more accurate to the measured result, they do not allow further analysis using the surface, as a functional equation can't be taken from a visual mesh. In the following section, interpolated surfaces will be discussed as a basis for developing surface model equations. 


\subsection{Effective Conductivity Surface Models and Simulation Analysis}

In comparison to the mesh surfaces in the previous section, the models presented here are simplified to a polynomial surface equation of best fit for the measured data. These surface formulas provide a model that can calculate conductivity as a function of both temperature and relative humidity. Each surface has a corresponding polynomial equation and list of coefficients in Appendix 8.2.

Similarly to the previous surfaces, the z-axis scale was selected in order to clarify the surface shape, and varies between materials. It is important to note the different z-axis scales between Fig 5.205.25 in order to accurately understand the values presented.

Fig 5.20, displays the polynomial model for material PU-A. This closed cell polyurethane material, through all testing remained relatively consistent in performance, rarely experiencing increases in conductivity of more than $10 \%$, only at the highest moisture levels and temperatures.

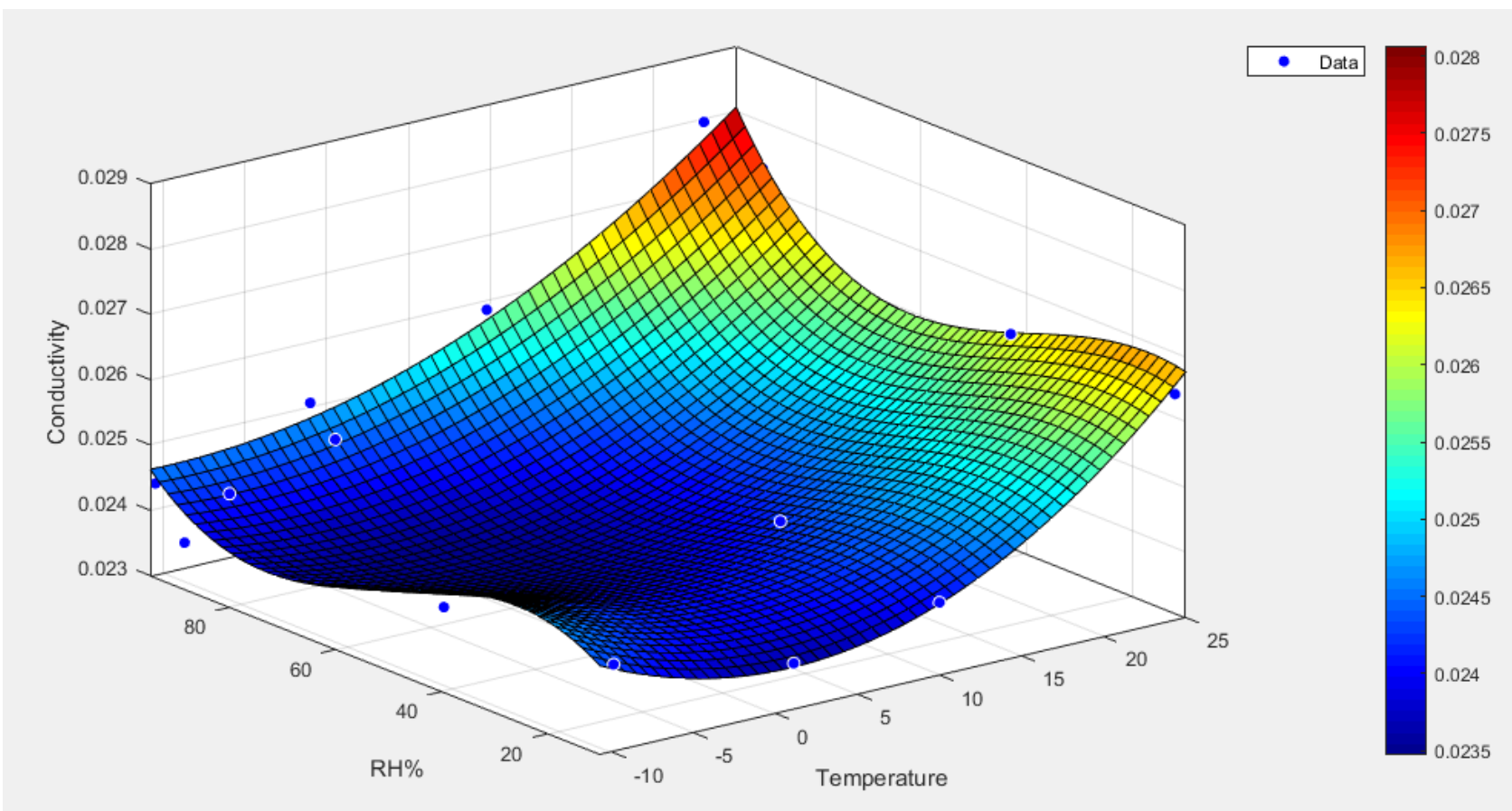

Figure 5.20: Effective Conductivity Model for Closed Cell PU-A 


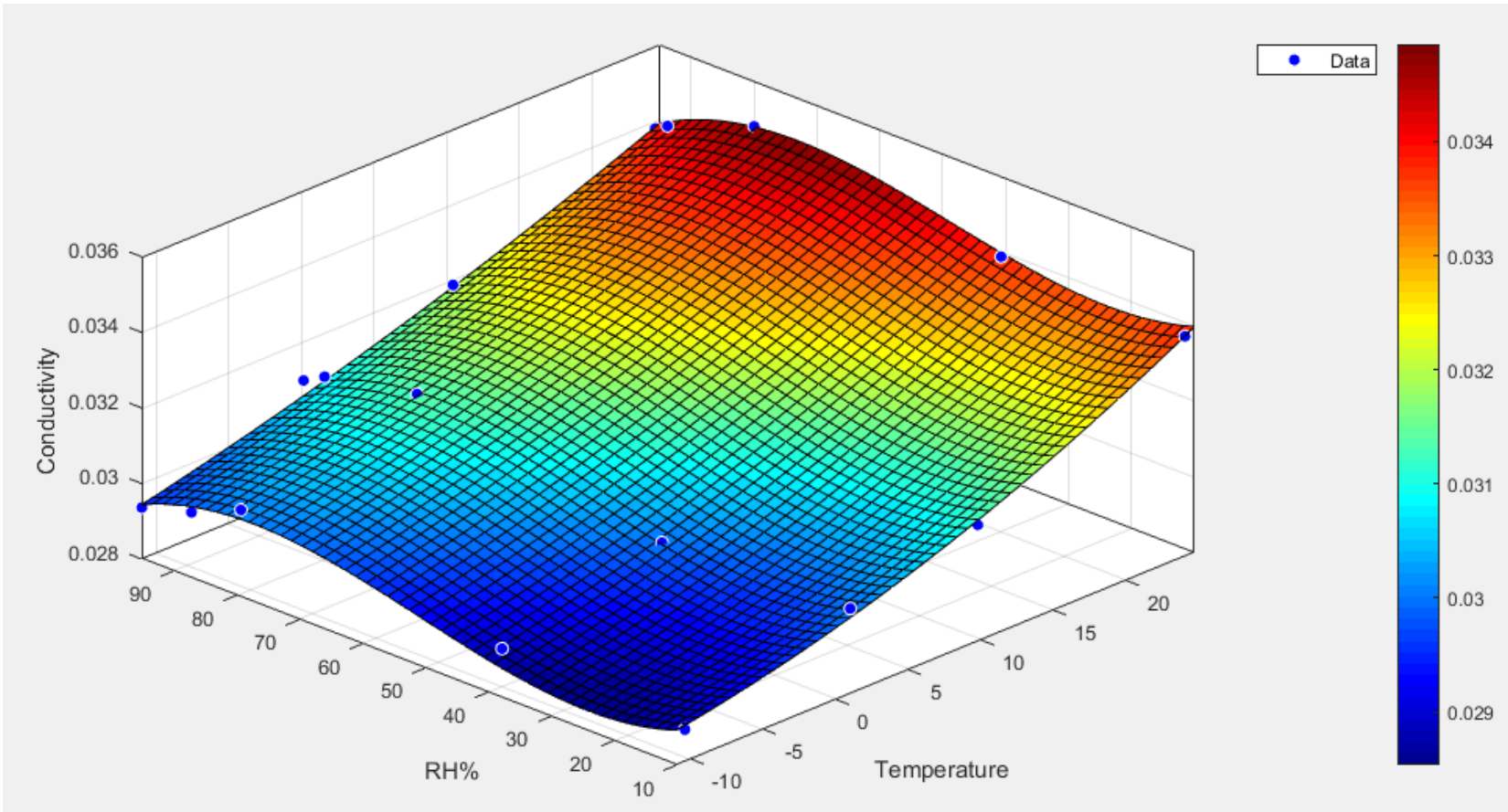

Figure 5.21: Effective Conductivity Model for Closed Cell PU-B

In Fig. 5.21, the second closed cell polyurethane PU-B presents a similar model to PU-A with a few key differences. In the case of PU-B, very high moisture levels were not as impactful as in the measurements for PU-A. This material also remains more consistent over a range of moisture levels, and experiences a fairly linear temperature dependent conductivity. 


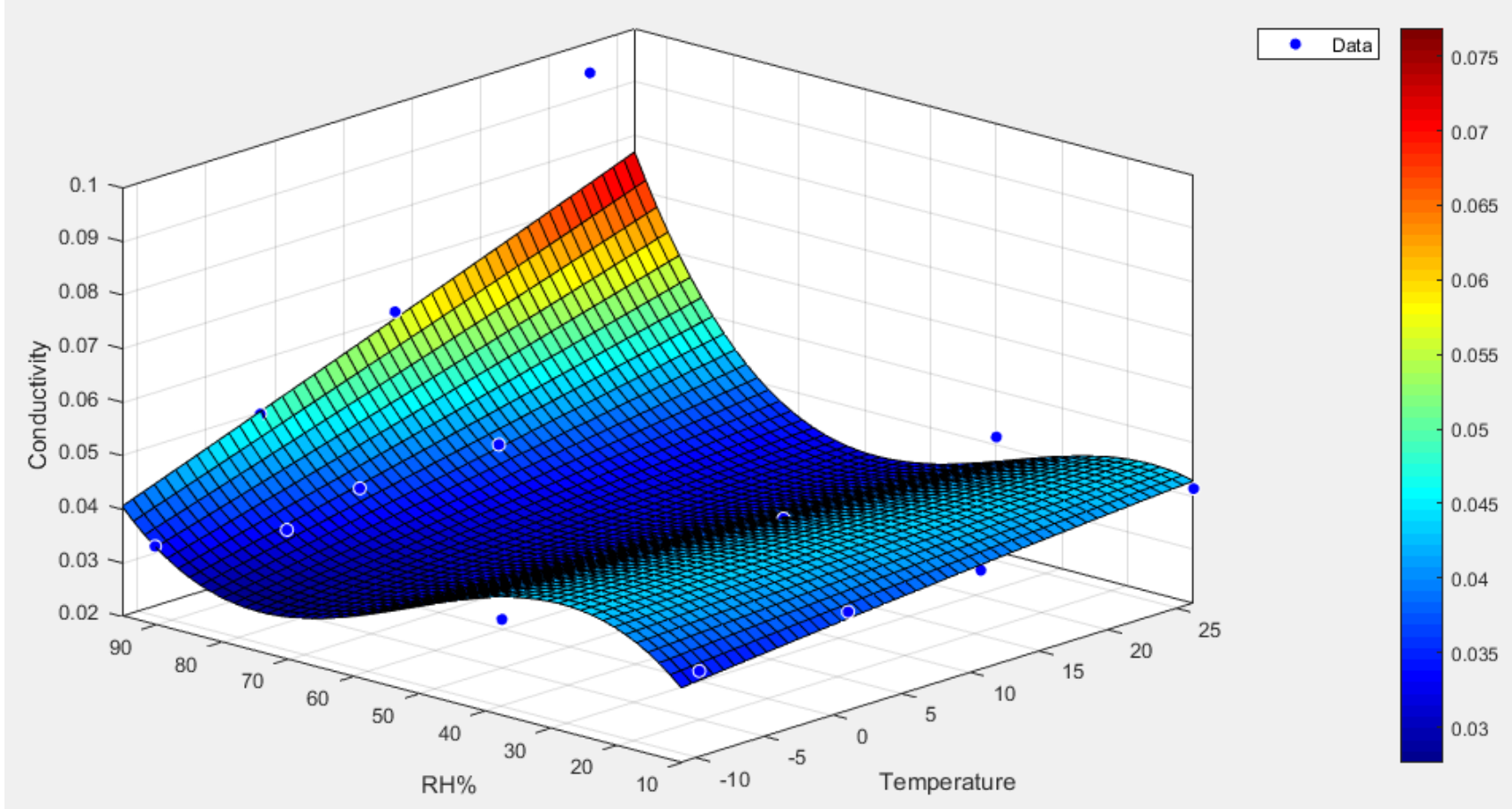

Figure 5.22: Effective Conductivity Model for Open Cell PU-C

In Fig. 5.22, the surface equation for the open cell foam PU-C is presented. There is a slight issue with the model creating a bump in the conductivity around $30 \% \mathrm{RH}$, while the measured data suggests that until $90 \% \mathrm{RH}$, the surface should be fairly flat, with a slight linear correlation with temperature. Beyond that issue, this surface indicates that the moisture dependent conductivity is the major factor in the performance of PU-C due to massive increases at high moisture levels. 


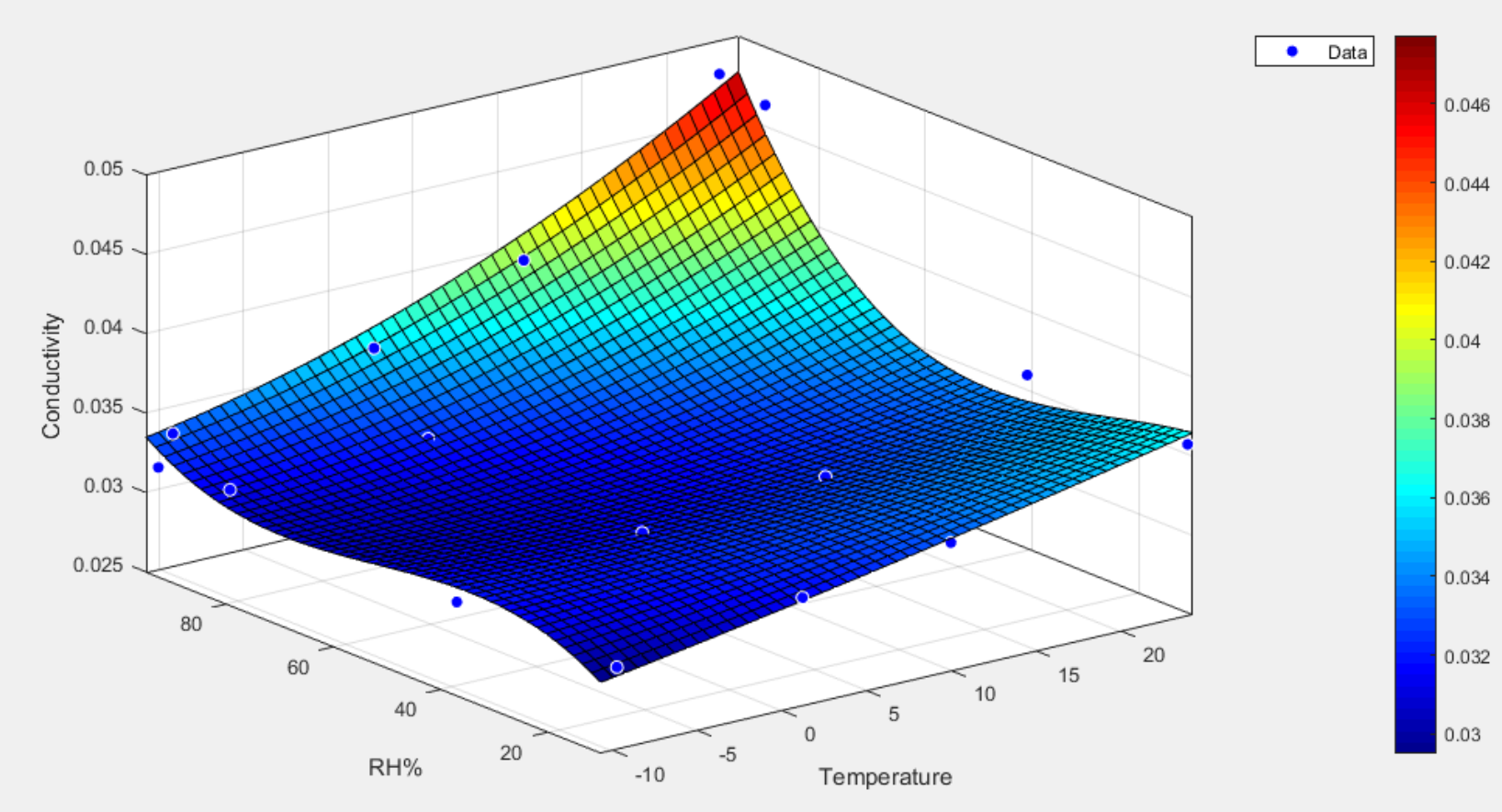

Figure 5.23: Effective Conductivity Model for Open Cell PU-D

The second open cell effective conductivity model presents a surface similar to what is expected from PU-C. The majority of the surface is relatively flat, with a slight linear relationship between temperature and conductivity. At the highest moisture levels, especially combined with higher temperatures, the increase in conductivity becomes very great. 


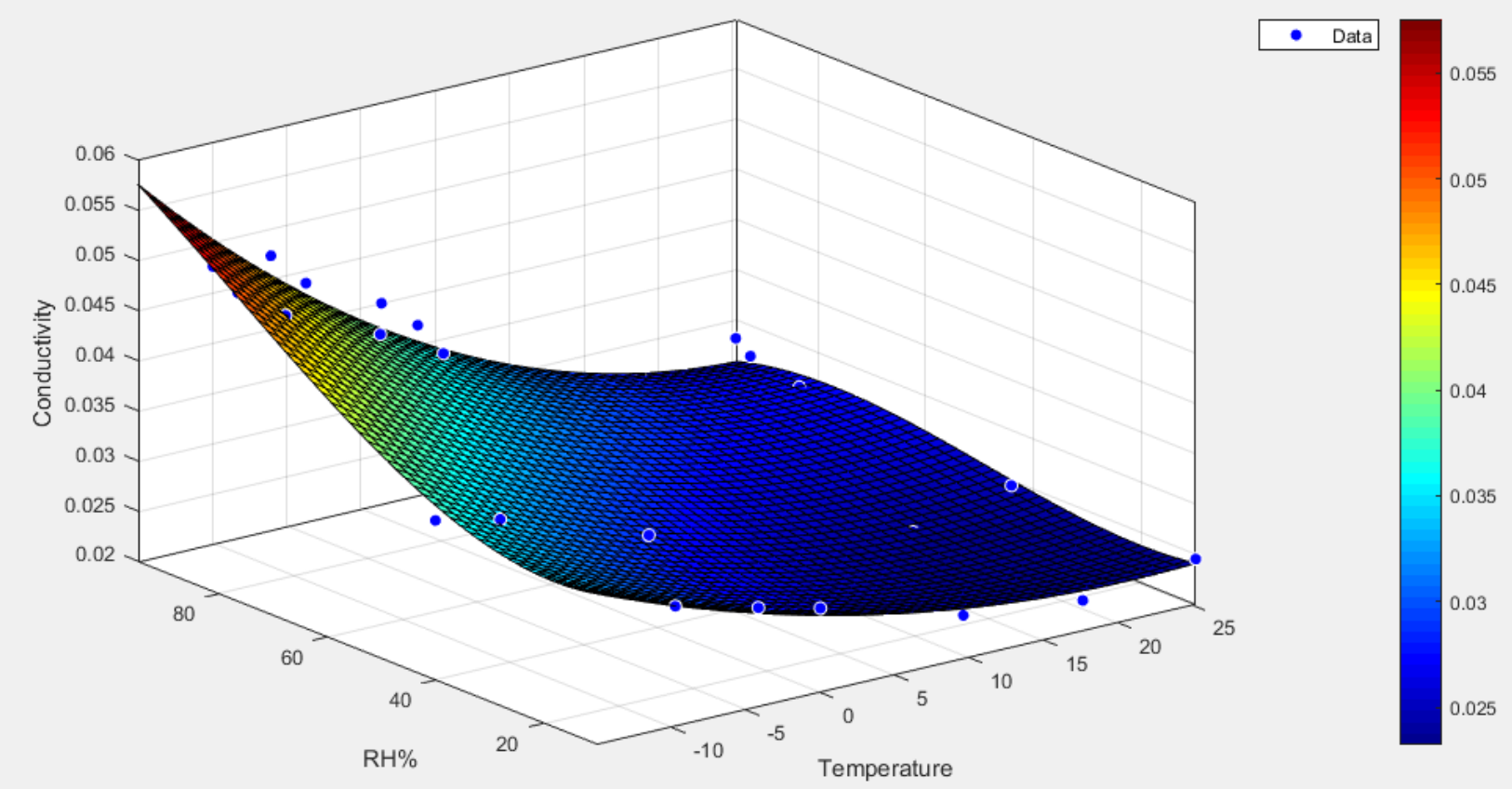

Figure 5.24: Effective Conductivity Model for Closed Cell PI-A

For the polyiso materials (Fig 5.24 - 5.25) both present very similar effective conductivity models. These materials have incredibly large increases in conductivity at low temperatures, and this effect is revealed in the model to be further increased when paired with high moisture levels. While all of the polyurethane materials held relatively consistent conductivities under most conditions other than very high humidity, both PI-A and PI-B experience massive increases in conductivity at low temperatures due to effects caused by blowing agents within the closed cells. 


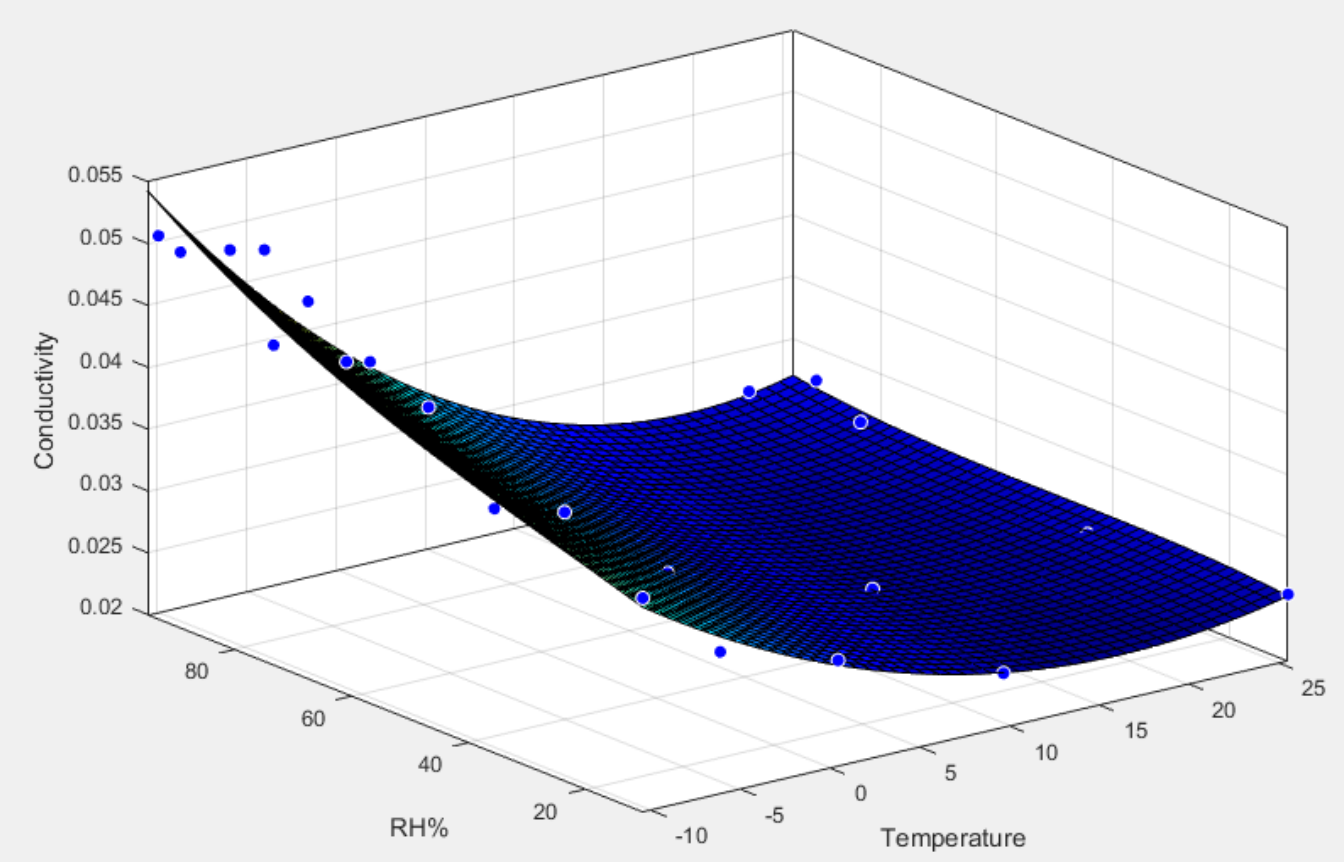

- Data

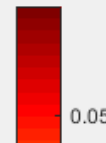

Figure 5.25: Effective Conductivity Model for Closed Cell PI-B

Through the development of these polynomial equations, the trends found in material behavior have been simplified enough to obtain a surface fit formula, while still providing a detailed enough function that the behaviours of the material under specific environmental conditions are accurately presented. Using the surface equations obtained through the MATLAB software from these surface models, a simulation result analysis similar to the analysis presented in section 5.2 has been completed.

In this simulation analysis, instead of comparing the performance of new and aged materials, the objective is to present a more accurate determination of the material effective conductivity as a function of both temperature and humidity, rather than temperature dependency alone as presented in section 5.2. In Fig 5.26-5.34, graphs present the temperature and humidity at the midpoint of the insulation layer in the assemblies, as well as the calculated effective conductivity over the course of a typical January and July in a Toronto, ON climate. Similarly to the graphs in section 5.2, the effective conductivity is calculated not only at the center point of the material layer, but at each face as well, in order to understand the range of effective performance through the layer. The solid lines present the 
calculated effective conductivity at the center point of the insulation layer, while the dashed lines present the calculated effective conductivity at each surface of the insulation layer. Additionally, for ease of comparing material samples, the y-axis is consistent between graphs, more clearly presenting the differences in how these materials are affected by both temperature and humidity.

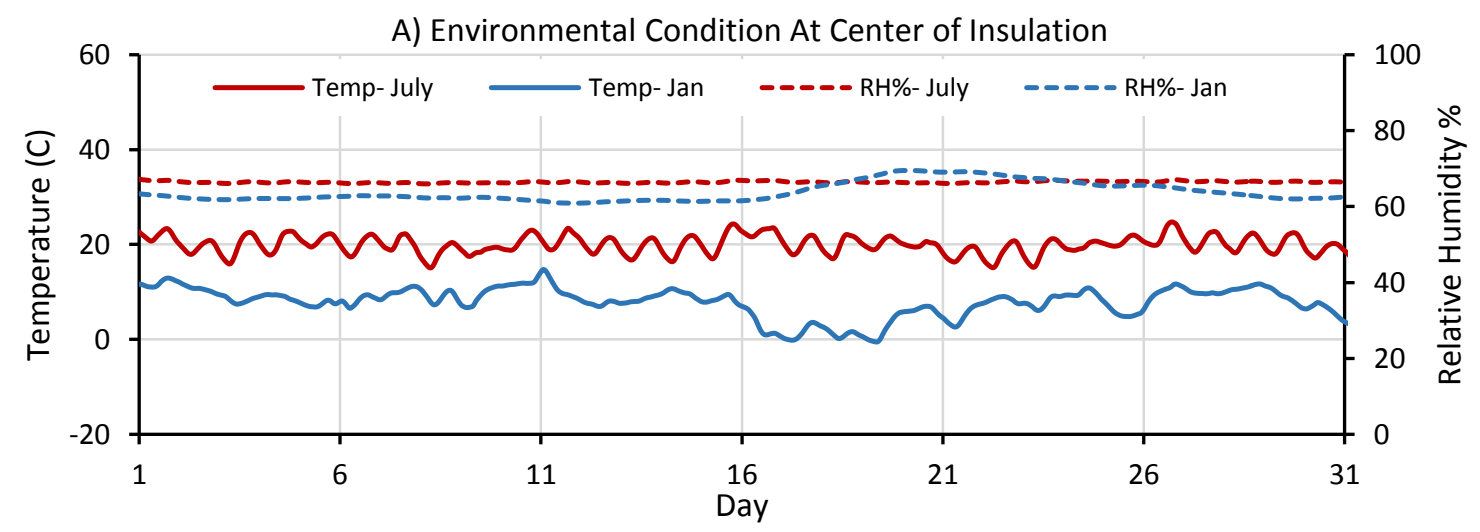

B) Effective Conductivity Of Insulation Layer

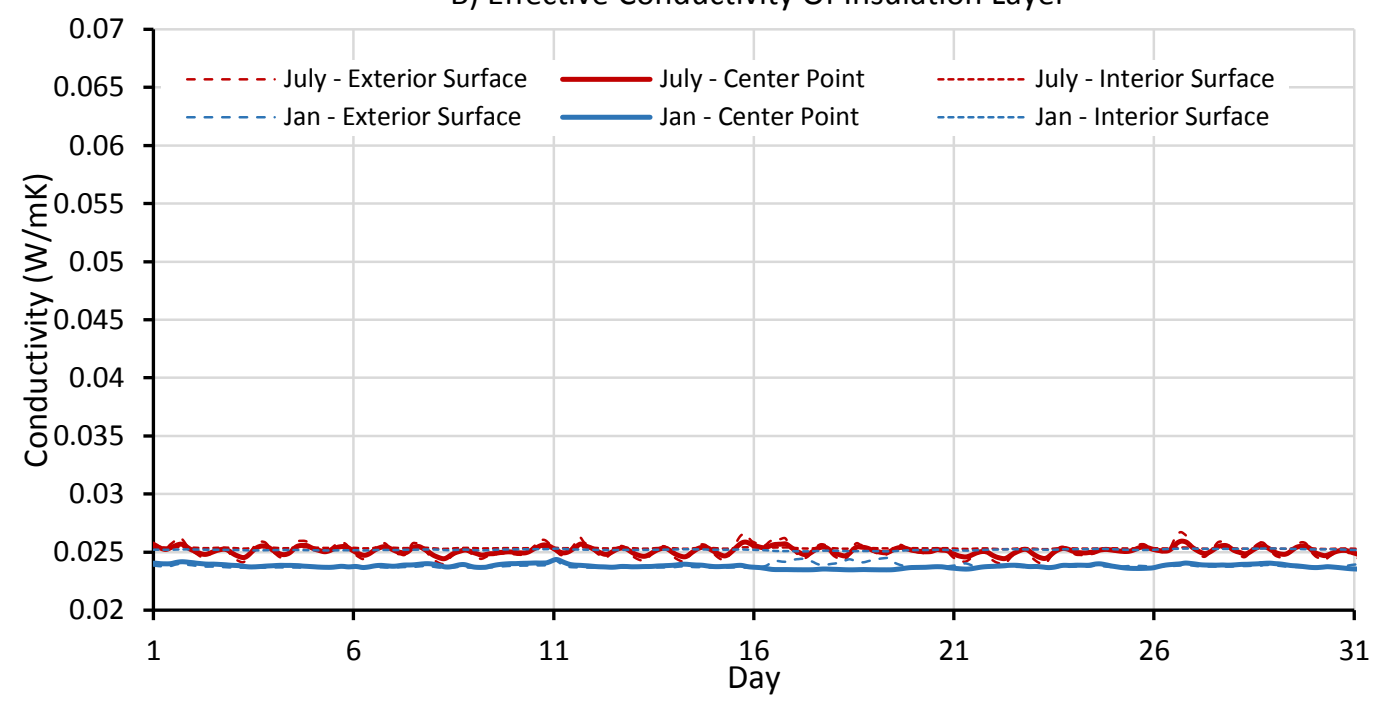

5.26: Effective Conductivity for Interior Insulation Layer of Material PU-A

In Fig 5.26, calculated effective conductivity of PU-A is presented based on a simulation using the basic wall assembly in Fig 5.2. It can be seen that when both temperature and humidity are considered, the effective conductivity of PU-A remains very consistent. The surface model for this material presented a very consistent performance that is minimally affected by temperature and humidity levels relative to the other materials. As shown in Fig 5.27, it is clear that the trend of consistent performance remains when material PU-A is used as an exterior insulation layer for the wall 
assembly in Fig 5.3. Even though in this simulation, it can be seen that the relative humidity levels surpass $80 \%$ in the winter, the performance of the material remains consistent.

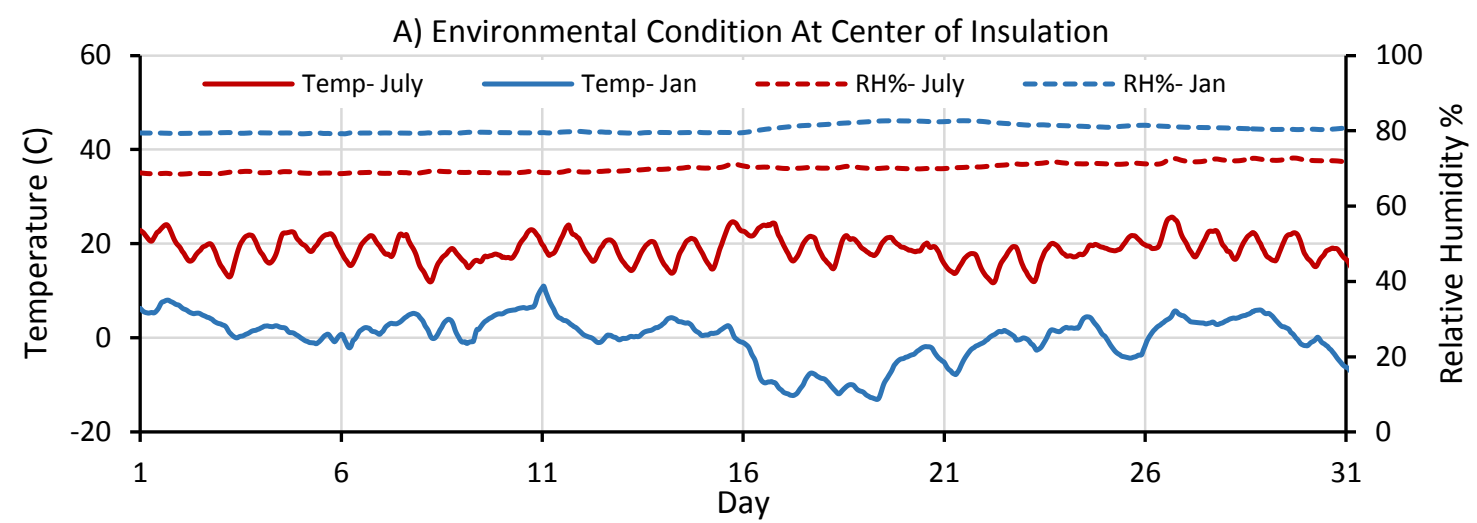

B) Effective Conductivity Of Insulation Layer

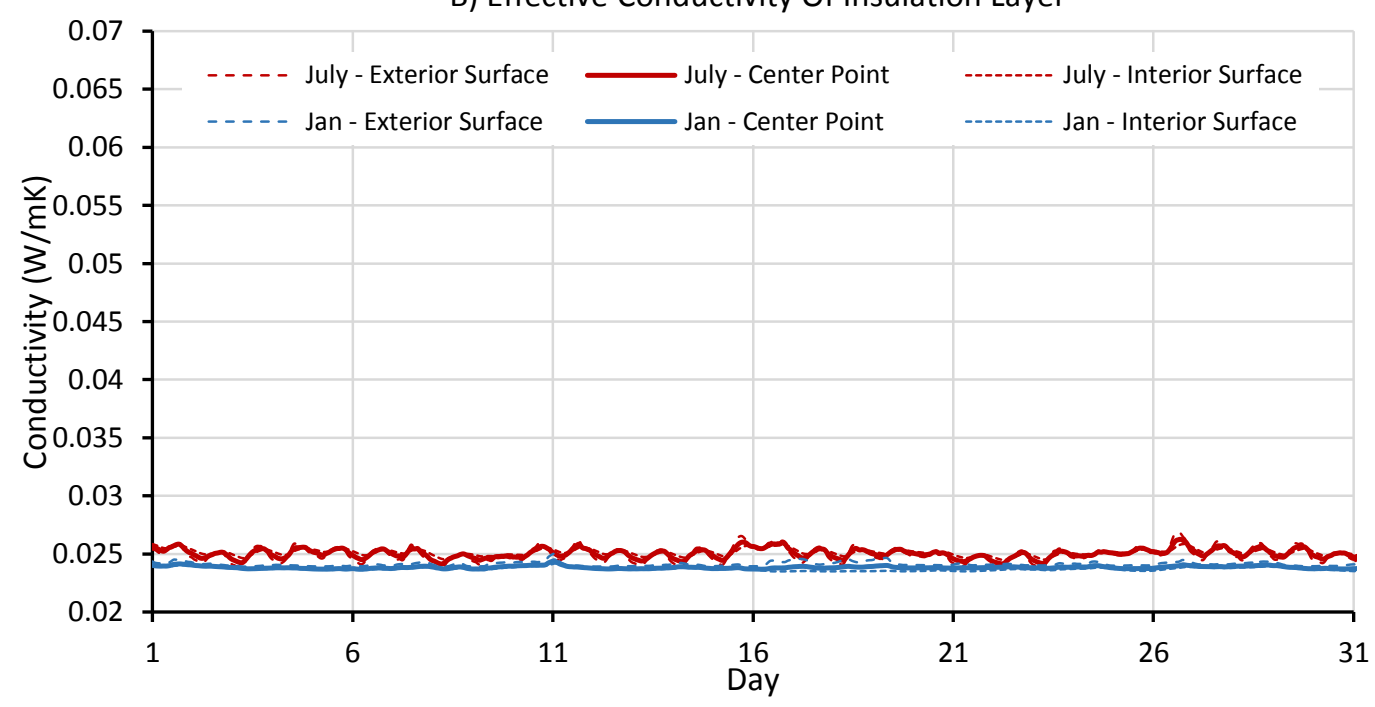

5.27: Effective Conductivity for Exterior Insulation Layer of Material PU-A 


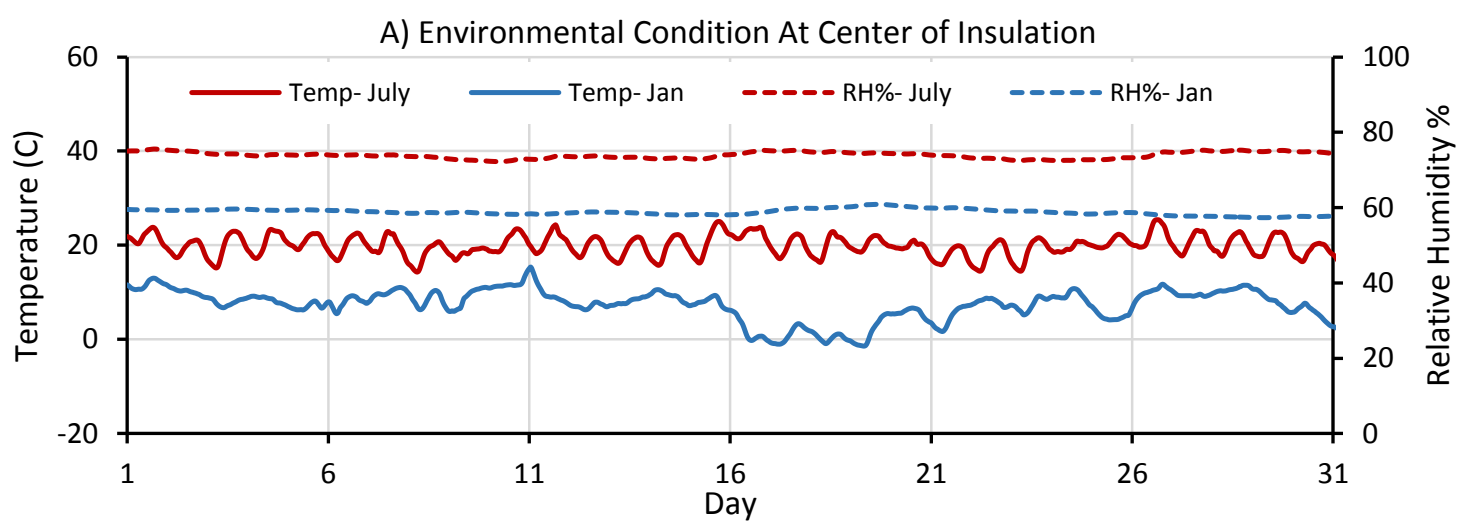

B) Effective Conductivity Of Insulation Layer

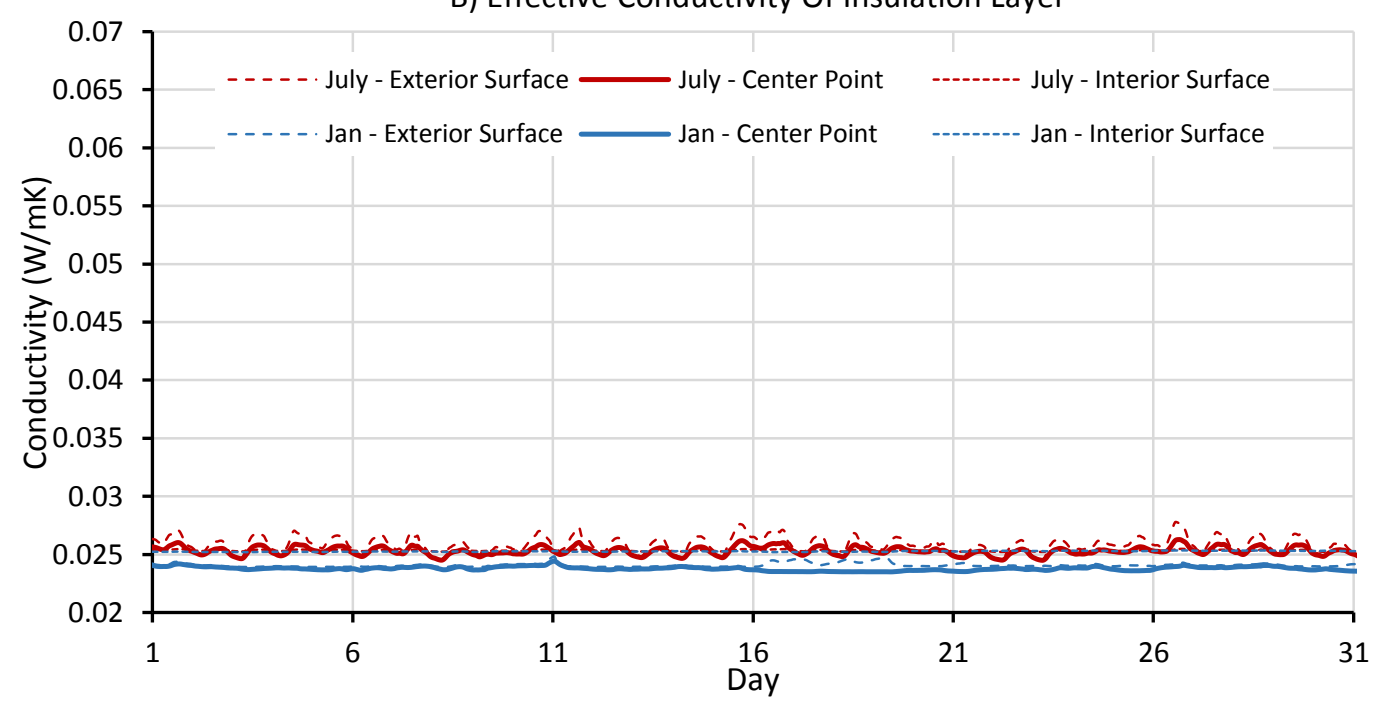

5.28: Effective Conductivity for Roof Insulation Layer of Material PU-A

The result presented in Fig 5.28, the simulation of the roof assembly (Fig. 5.4), unsurprisingly reveals very consistent performance from material PU-A. Through all three assemblies, it is seen that PU-A has minimal fluctuation in performance, and the range of performance between the interior and exterior surface at any given moment is quite minimal, evidenced by the fact that the dashed lines presenting surface values are very close to the values of the solid line presenting the center point conductivity value. 
As a contrast to the result of PU-A, when taking into account both temperature and humidity, the results of PU-D, an open cell polyurethane, a larger delta of performance is seen due to the effects of high moisture levels. In section 5.2, it was shown that when considering temperature dependent conductivity alone, the material was calculated to have a relatively consistent conductivity. When taking into account humidity level as well, it is clear that the open cell material is greatly affected by changing moisture levels, especially the outside surface of the material. In Fig 5.29, the basic wall assembly simulation reveals that especially in the summer, the humidity levels are increasing the moisture levels in the material, causing an overall decrease in performance, specifically towards the outer layer, where moisture will infiltrate the material quickly.

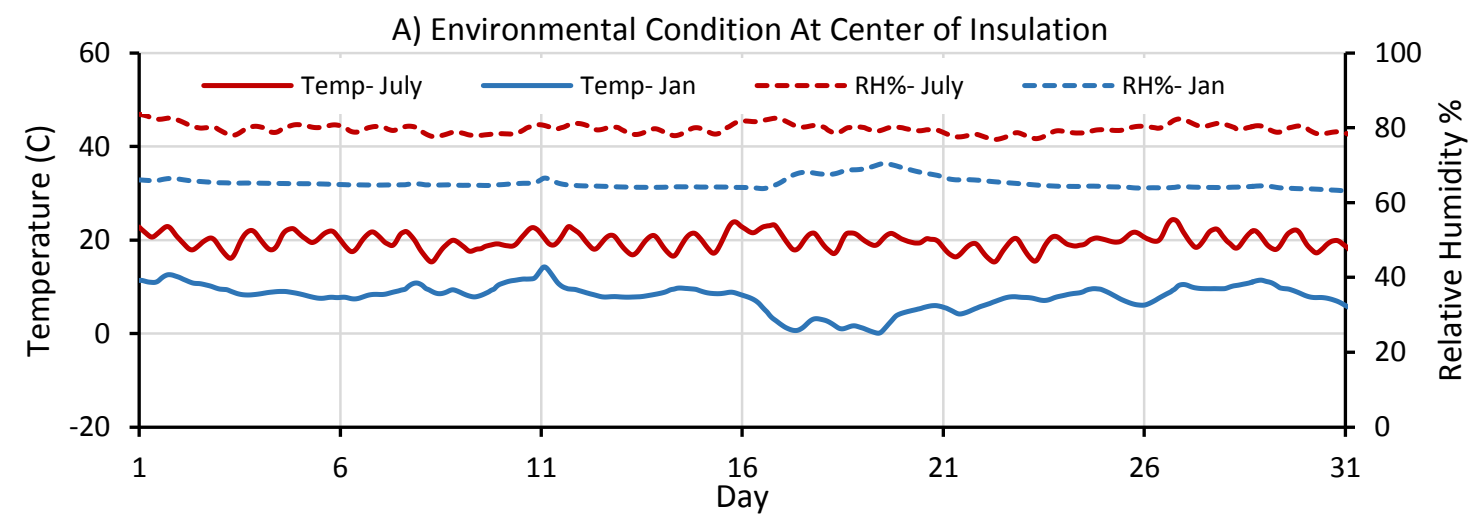

B) Effective Conductivity Of Insulation Layer

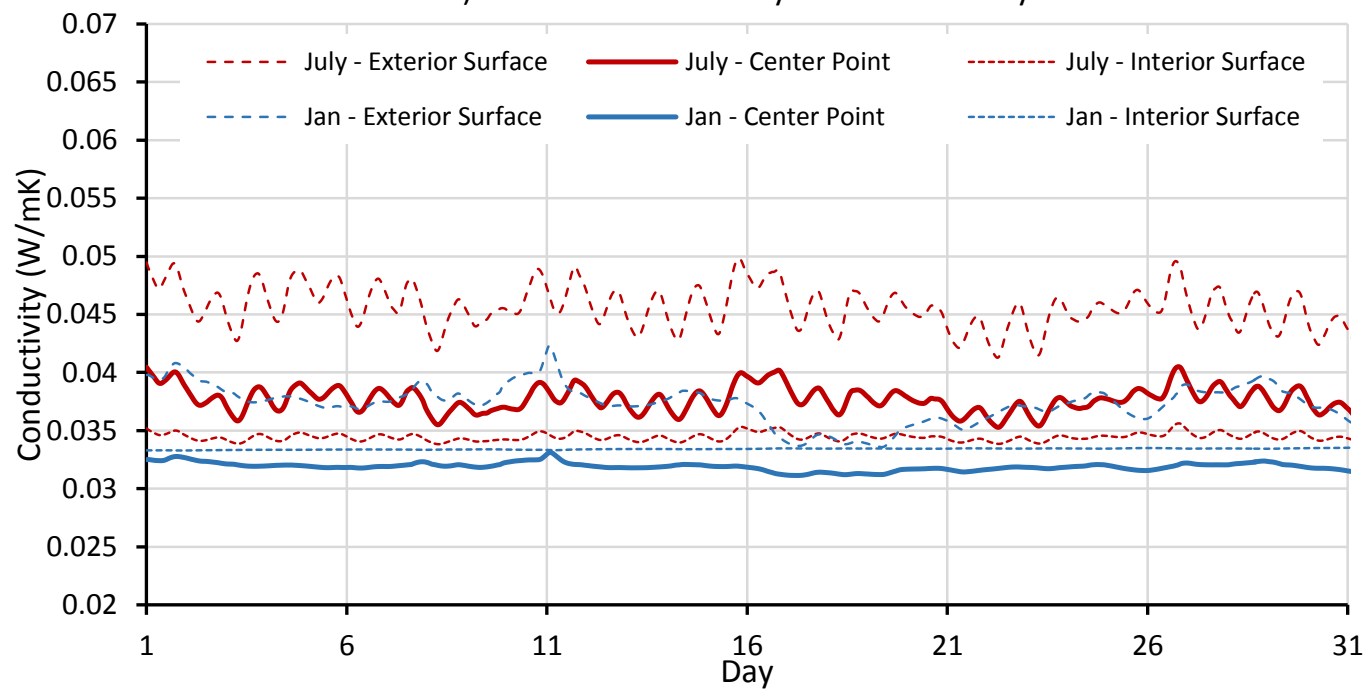

5.29: Effective Conductivity for Interior Insulation Layer of Material PU-D 
The effect of moisture increasing the conductivity and decreasing the consistency in performance is further exaggerated when the open cell material PU-D is used as an exterior insulation layer. It was measured that for most of the summer, the humidity level towards the outside surface of the material was constantly above $95 \%$, allowing much more moisture to infiltrate the foam. In comparison to Fig 5.29, the result of the exterior insulation simulation in Fig 5.30 shows that the increased moisture levels affect the material over the entire layer, not just towards the exterior surface, evidenced by the interior surface conductivity matching the trends seen at the center point and exterior surface much more closely than in Fig 5.29, where the interior surface still remained relatively consistent.

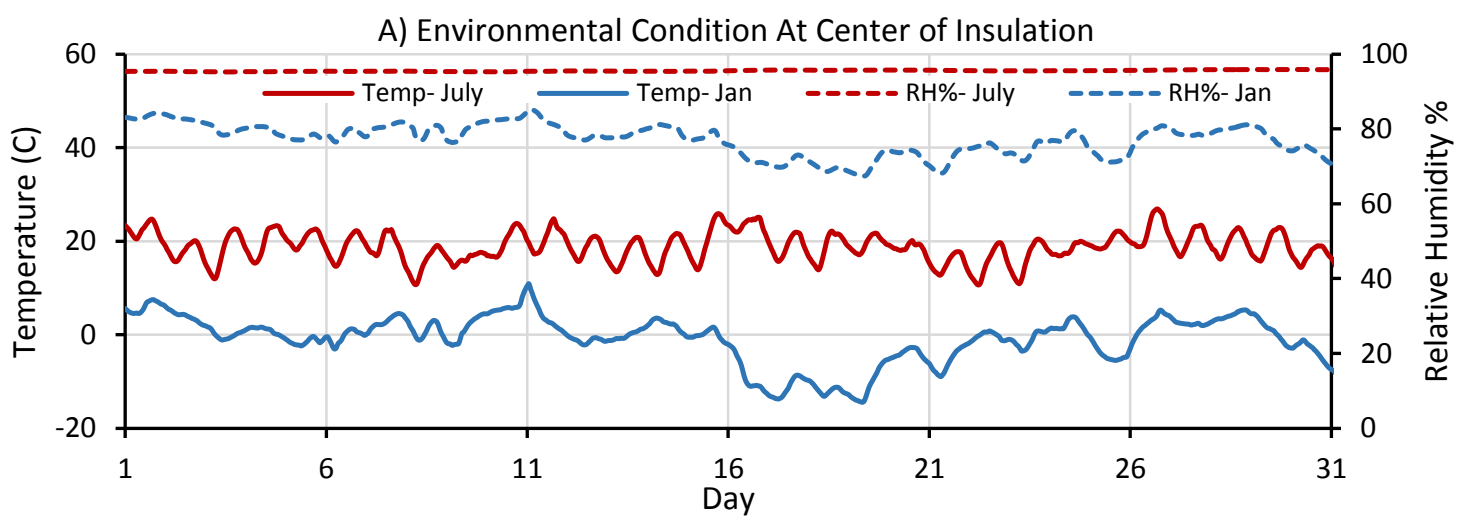

B) Effective Conductivity Of Insulation Layer

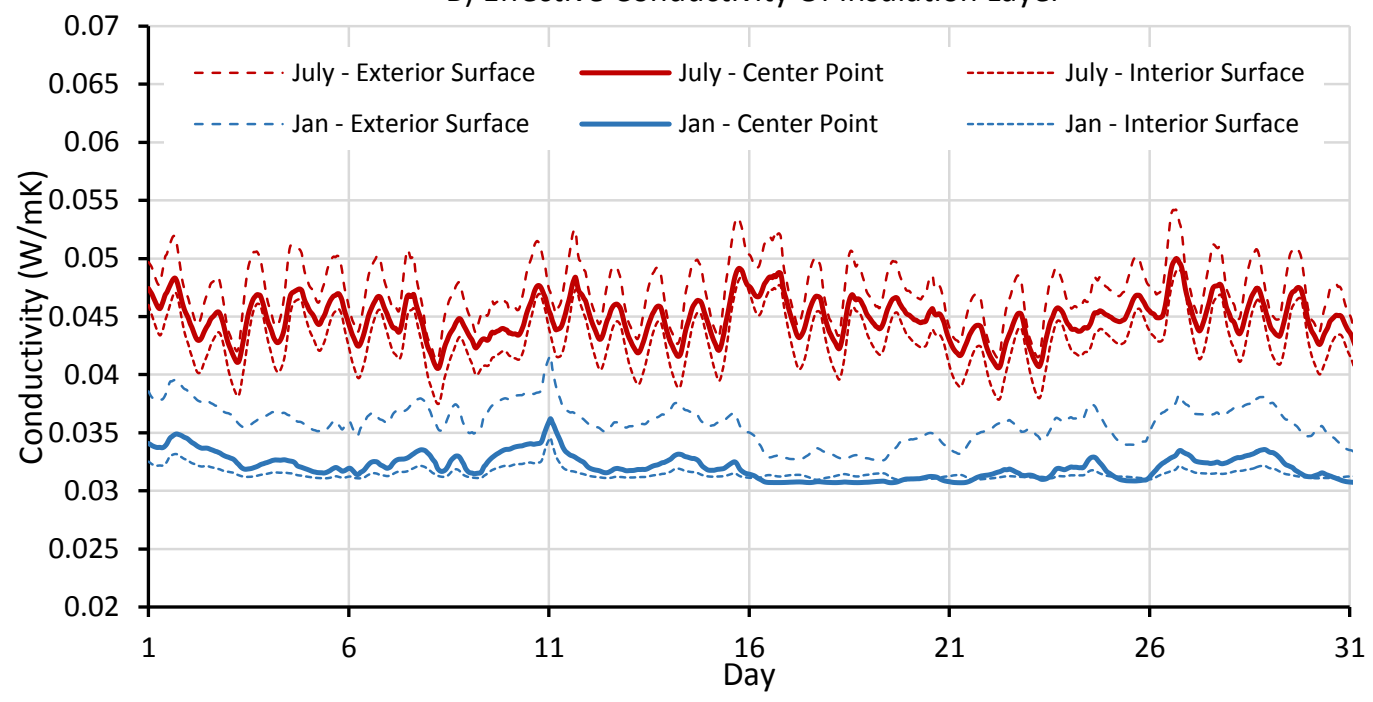

5.30: Effective Conductivity for Exterior Insulation Layer of Material PU-D 


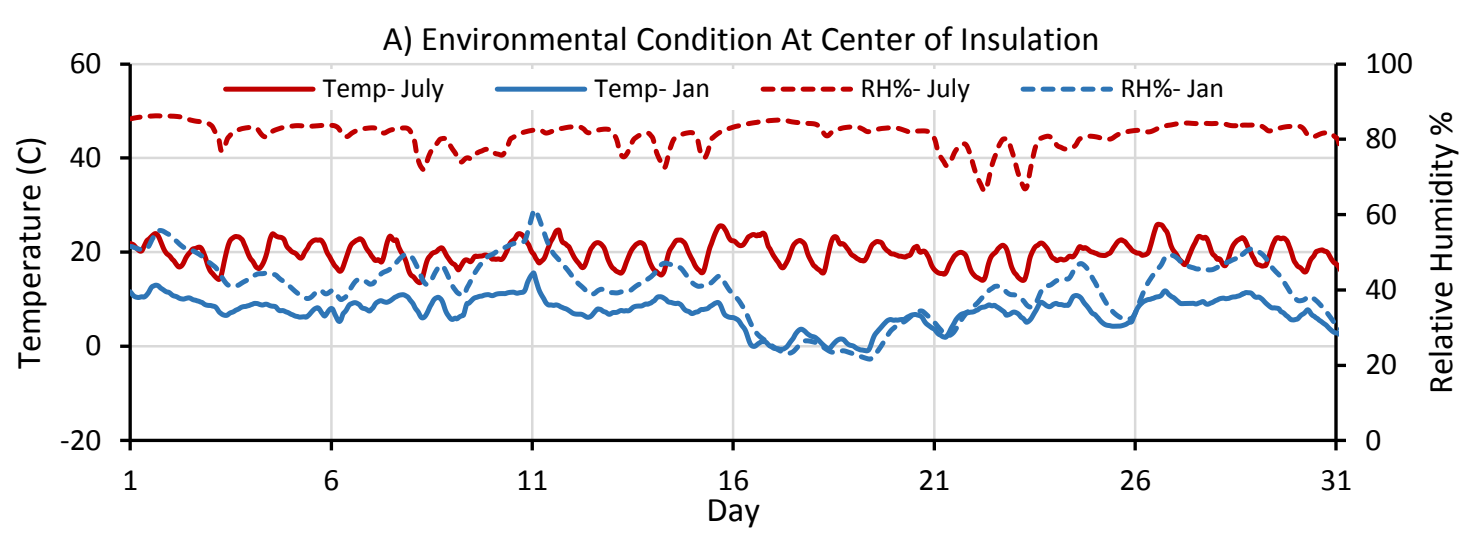

B) Effective Conductivity Of Insulation Layer

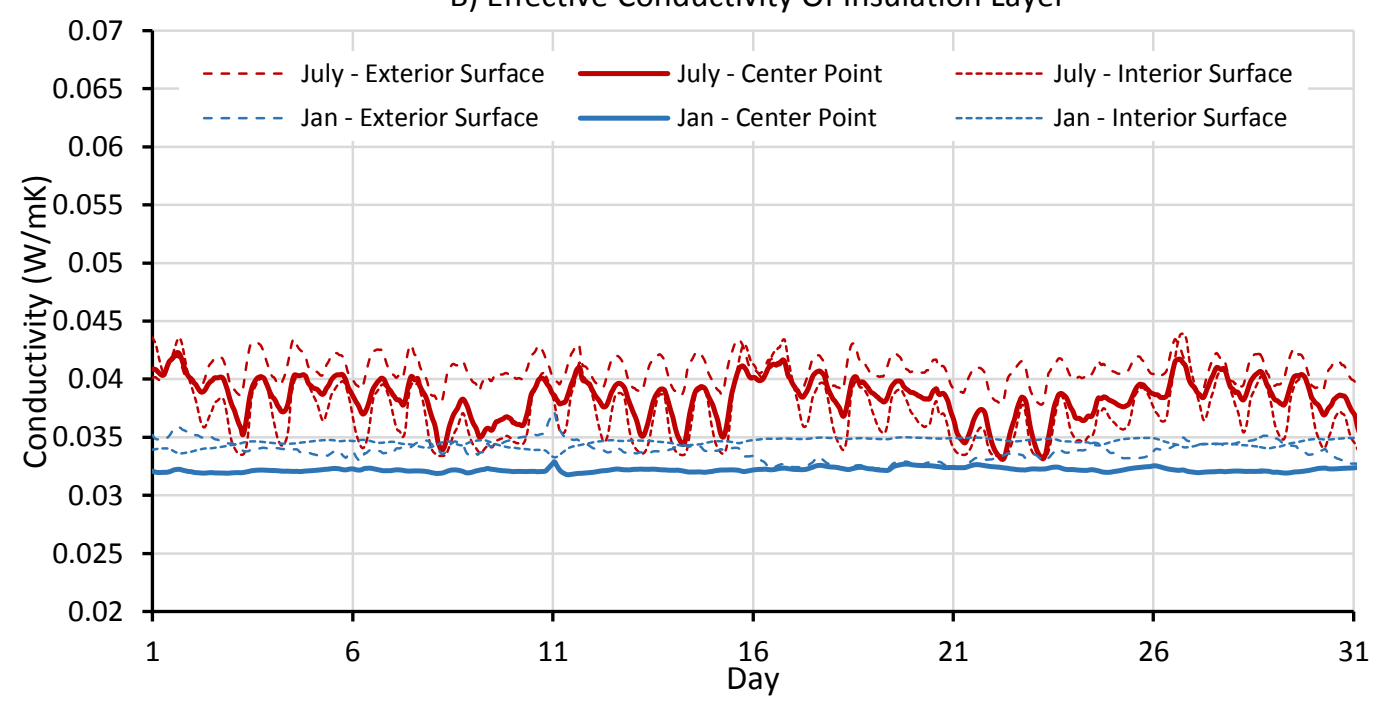

5.31: Effective Conductivity for Roof Insulation Layer of Material PU-D

While the roof assembly simulation in Fig 5.31, shows that the moisture levels are not quite as high as in the exterior insulation simulation (Fig 5.30), the conductivity of the material is much more volatile than the closed cell PU-A. It is important to note, that generally, open cell foams are not generally used in locations where high moisture levels may be present due to the fact that moisture will infiltrate the material so easily and compromise the performance. While the use of open cell foam as an exterior or roof insulation is generally ill-advised, it is valuable to see the impact that moisture has on the performance of PU-D, reinforcing the reasoning behind avoiding use in these types of scenarios. 


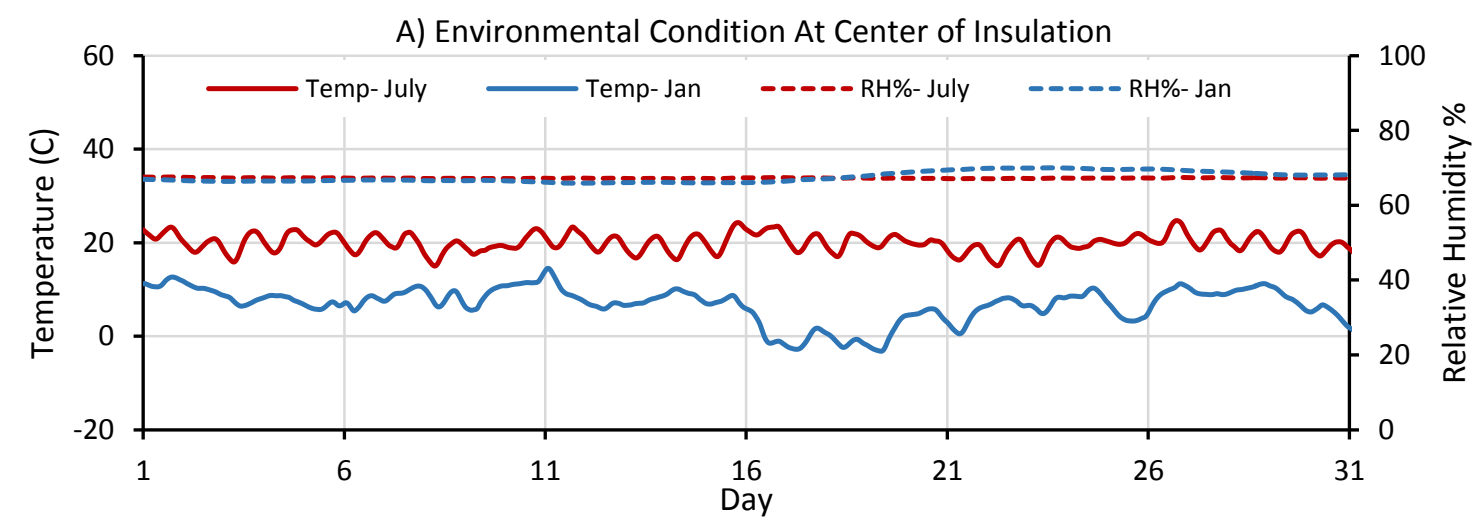

B) Effective Conductivity Of Insulation Layer

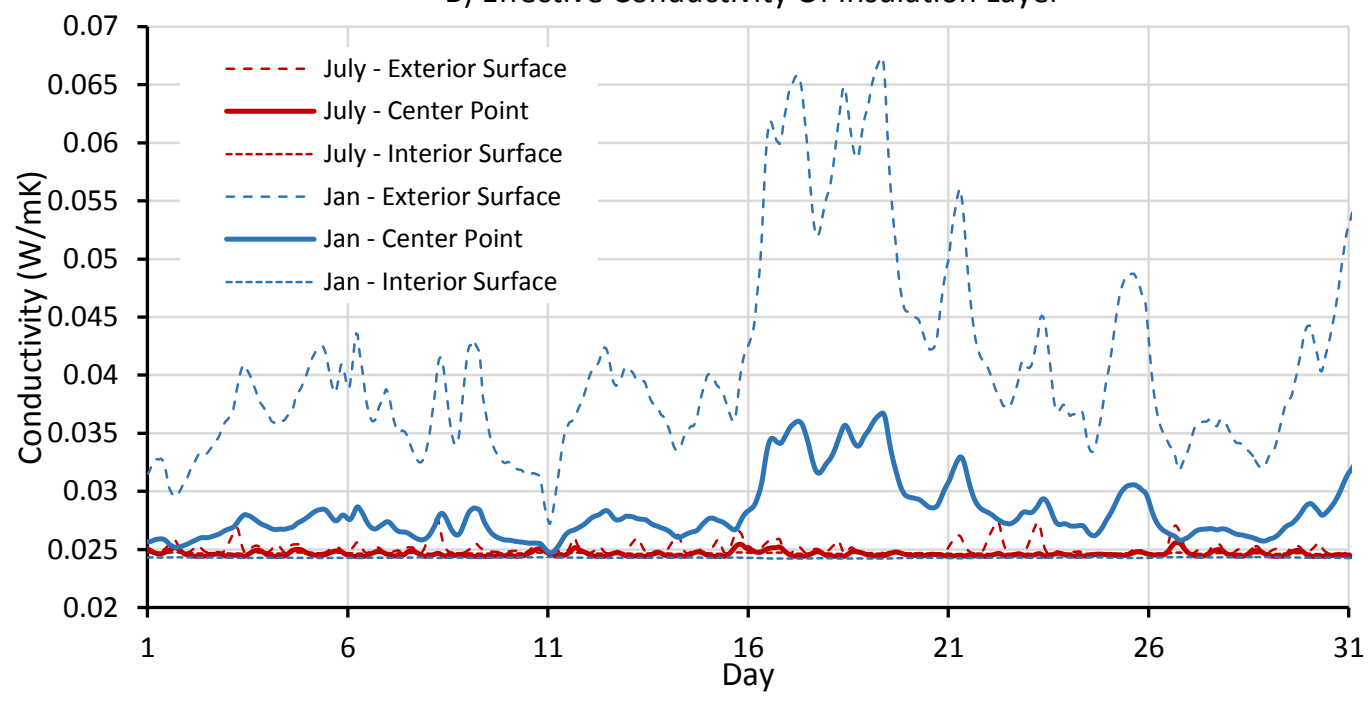

\subsection{2: Effective Conductivity for Interior Insulation Layer of Material PI-B}

For the polyisocyanurate material, very similar trends are seen when taking into account both temperature and humidity, compared to the results in section 5.2 which only consider temperature. The major difference is that while, the trends are similar, the extent of the effect on performance is greatly increased. During the coldest points in January, the calculated conductivity exceeds $150 \%$ increase compared to the performance in summer as shown in the simulation of a basic wall assembly using PIB in Fig 5.32. The large range between the solid line and the dashed line above in January, suggests that about half of the thickness from the center to the exterior surface of the insulation layer will be performing far worse than expected. This is seen to an even larger extent when the material PI-B is used as an exterior insulation layer (Fig 5.33). In this simulation, it is calculated that even the interior 
surface of the insulation is greatly affected by the combination of cold temperatures and higher humidity. The material conductivity at the center of the insulation layer is over $60 \%$ higher than the relatively consistent summer performance for nearly all of January, with some points spiking up to $100 \%$ higher conductivity at the center point and a near $200 \%$ increase in conductivity at the worst case towards the outer surface.

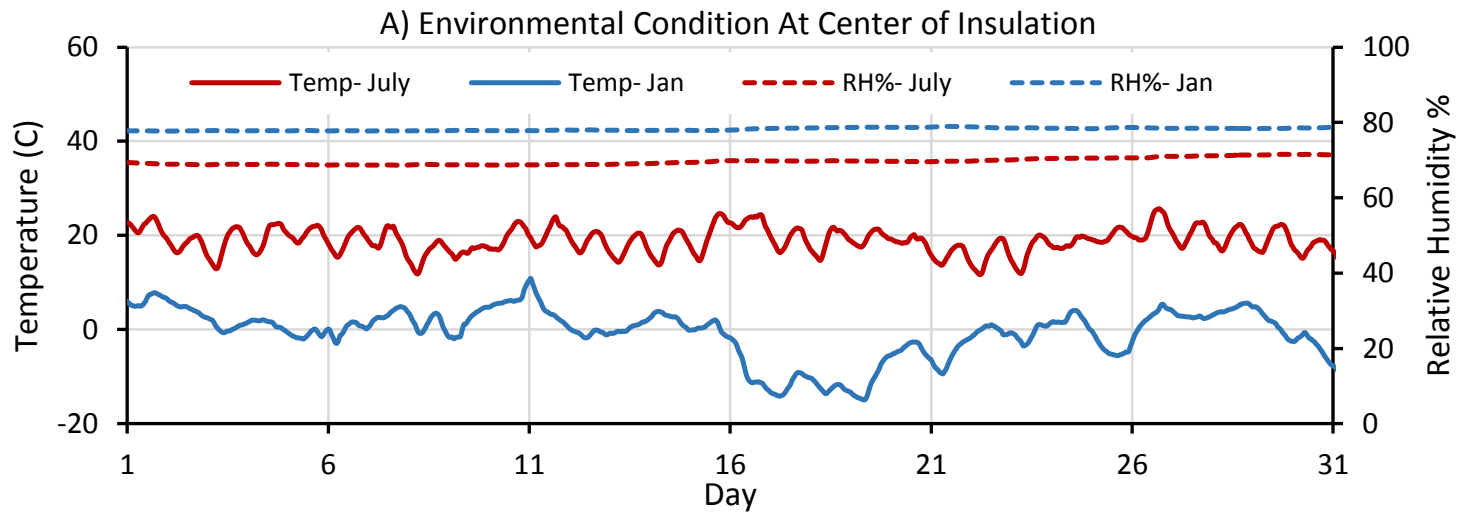

B) Effective Conductivity Of Insulation Layer

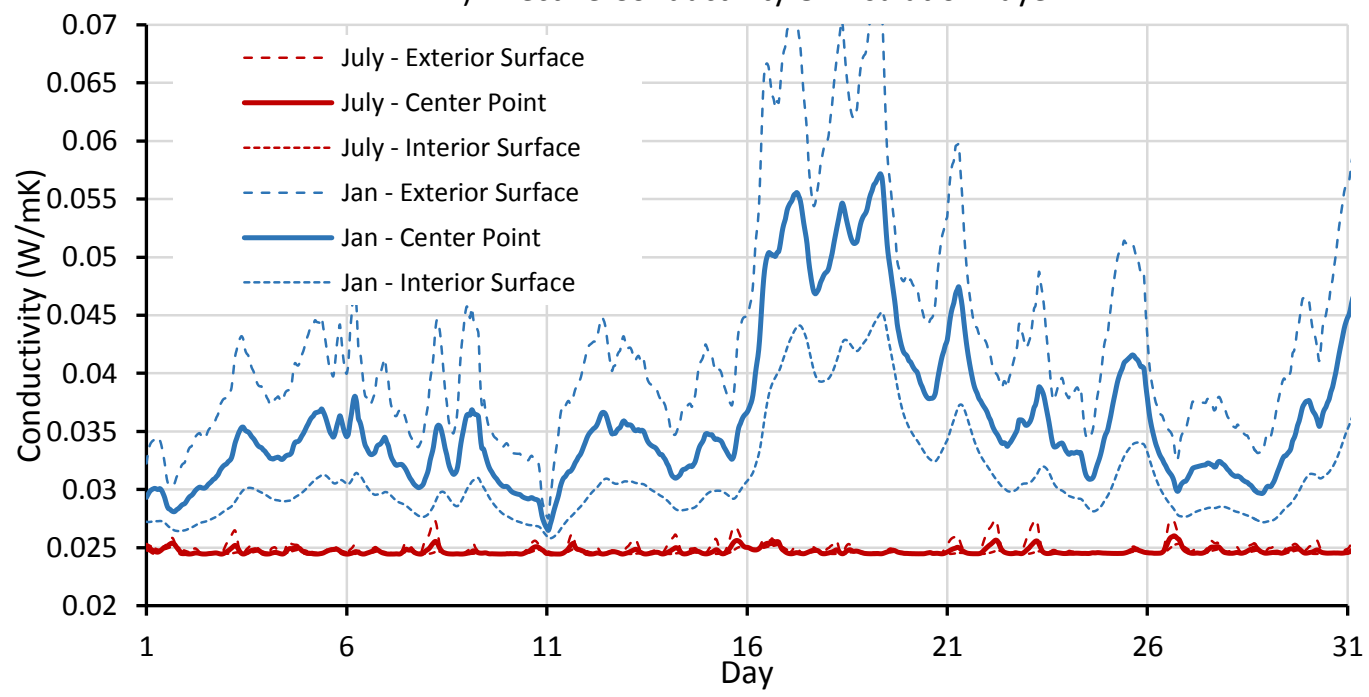

\subsection{3: Effective Conductivity for Exterior Insulation Layer of Material PI-B}




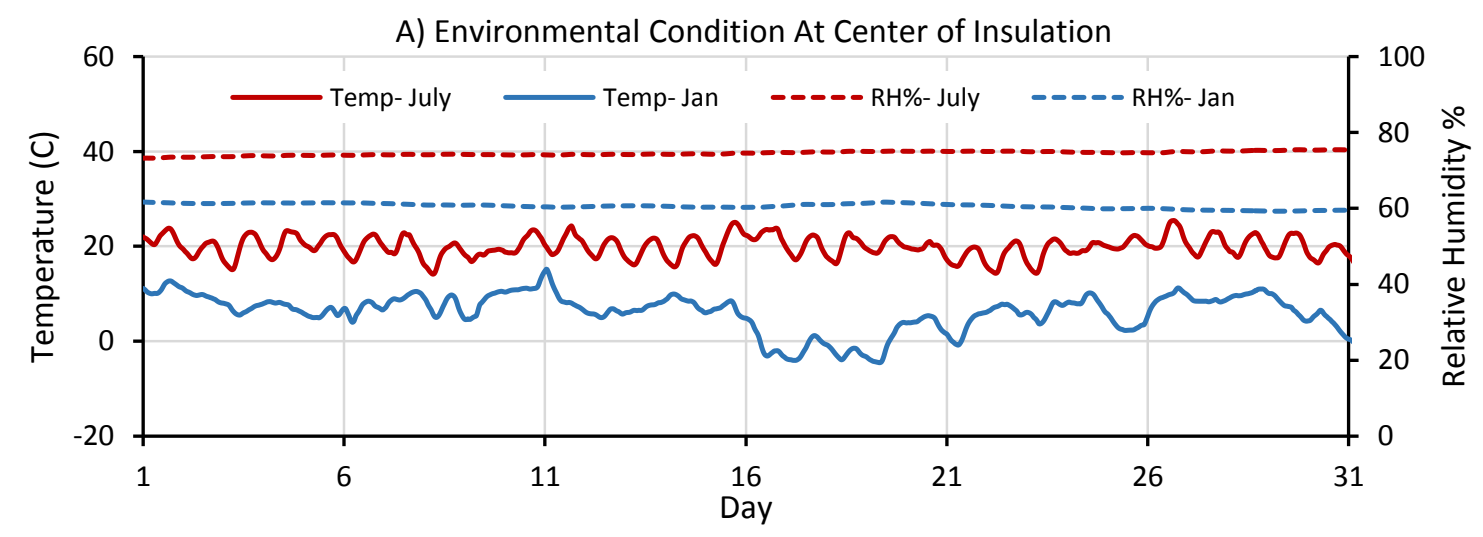

B) Effective Conductivity Of Insulation Layer

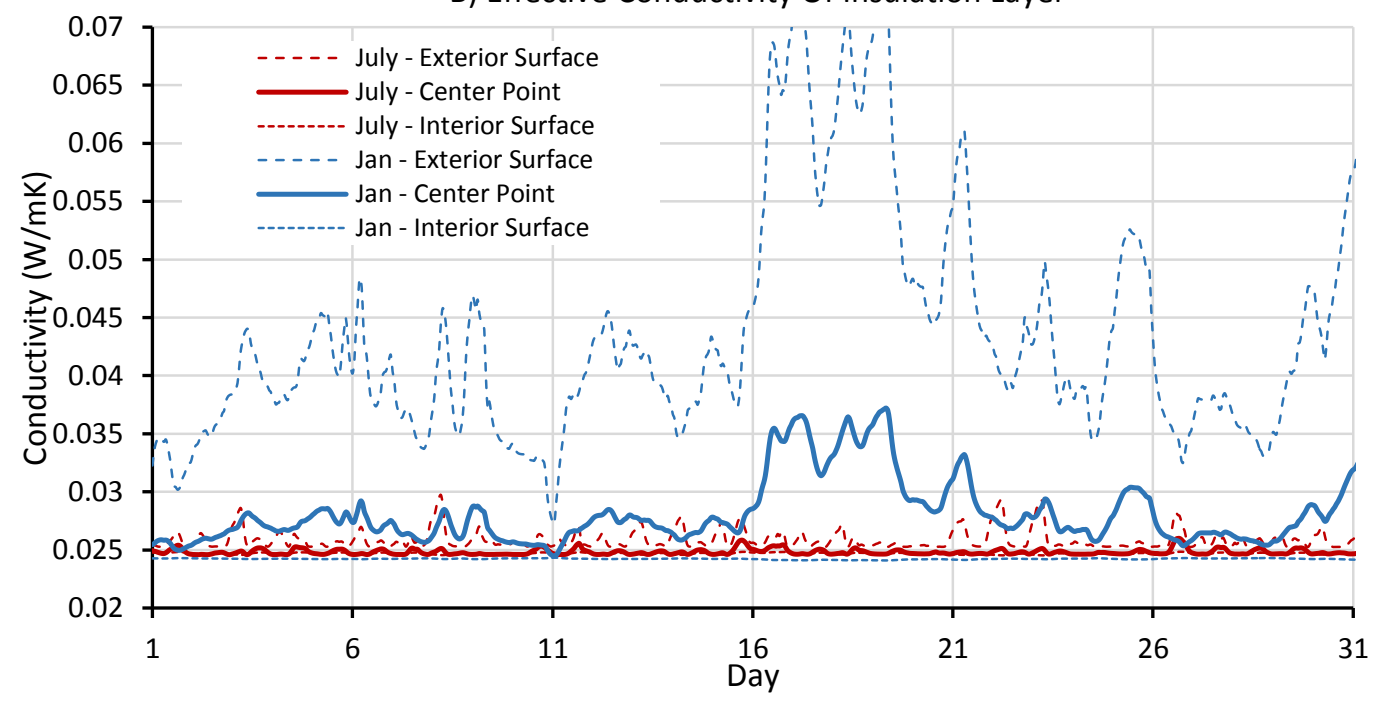

5.34: Effective Conductivity for Roof Insulation Layer of Material PI-B

In all three simulations using the PI-B material, the winter causes high variability in the performance, while the material is relatively consistent during the summer environment. This is a major issue for two reasons; in a cold climate such as in Toronto, ON, the most important time of the year regarding insulation performance is in the winter heating season. Secondly, due to the standards for determining advertised material values, the material performance at lower temperatures is often ignored by or unknown to designers and consumers. 


\subsection{Conclusion}

Singular conductivity values of insulating materials are typically used in building design and assessment. However, the thermal conductivity of insulating materials is affected by the environmental operating conditions. Attention has focused on foam insulation such as polyurethane and polyisocyanurate, which exhibit less regular behavior, and experience larger changes in performance under varying conditions. Using singular thermal resistance values in design will result in actual building envelope performance that will vary from the calculated level, as it has been shown both through lab measurements and hygrothermal simulations of the results. The results of simulations have also shown the importance of understanding how a material performs within a given assembly, emphasizing the importance of simulating assemblies with accurate data for the purposes of high efficiency building. Decisions and calculations made during the design phase must accurately reflect material properties in order to have buildings performing at the expected level.

In building design, the type of measured values presented in this thesis should be far more accurate in determining the actual performance of a given material in specific climate conditions than if a singular value was given for a material conductivity, which would only provide a snapshot of the potential performance range. Through these findings, it can also be understood that there is no strict rule for how a given material will perform under a range of environmental conditions. Models developed through lab testing with one material cannot be used as a general rule applied to other materials. This emphasizes the idea that in simulations for designs where a high degree of accuracy is required, software calculations must be carried out with data accurate to the specific materials used, not only under the assumption of a single conductivity value or even an assumed linear effective conductivity, but with the understanding of how the material will be affected by the environment (temperature and humidity), as well as aging effects. 
Through the development of 3-D effective conductivity models, it is clear that between different types of materials, the thermal behaviour varies greatly when considering the effects of temperature, moisture and aging effects. Design based on accurate modelling would aid in reducing building performance failures in designs where a high degree of accuracy is required in building systems, such as Passive House, and Net Zero design.

This thesis aims to start a critical discussion about how the impact of temperature, moisture levels and material aging on the effective thermal conductivity affect (foam) insulation performance in building envelopes, and how issues that may arise from the dynamic effective conductivity may be mitigated in the design phase through the use of more accurate simulations taking into account extensive laboratory measured data. 


\section{Part 7: Limitations and Future Development}

Limitations of this thesis include equipment limits and constraints, material availability, software uncertainties, and the need for further software analysis.

Two aspects of equipment limitation have had an effect on the reported results. The heat flow meter used for measuring material conductivity experienced issues when testing at low temperatures. This meant that the plates of the heat flow meter could not feasibly be reduced below approximately $-15^{\circ} \mathrm{C}$ without risk. Additionally, some of the test chambers had limitations on the consistency of environment set points. The custom environmental chamber used for moisture exposure fluctuated up to $+/-2.5^{\circ} \mathrm{C}$ and $+/-4 \% \mathrm{RH}$ from the set points. Additionally, other issues such as dripping from the chamber lids may have been a factor in the moisture exposure tests.

Some material samples were only possible to obtain in small quantities, meaning few extra samples were available of some materials. The availability of samples, along with limited space in the test chambers and equipment, impeded the ability to test many samples at once for greater sample size in the data sets, rather than relying on 1 sample per test, where outlier results may cause some unclear understanding of the material behavior.

In hygrothermal simulation using WUFI software, uncertainties in the simulation process limit the understanding of exactly how the temperature and humidity at each given point in the wall assembly are calculated, as well as how the simulation process interpolates the input temperature and moisture dependent conductivities from the custom material files.

Additionally, when assessing the simulation results in the context of measured effective conductivity, only temperature was taken into account when calculating the effective conductivity based on the temperature dependent curves before and after aging in section 5.2. Scheduling constraints prevented the ability to conduct a full moisture investigation on the samples that underwent elevated temperature accelerated aging, therefore only allowing the comparison of temperature dependencies before and after the accelerated aging process. While these results presented valuable information 
regarding the effective performance as a function of temperature as before and after aging effects, the results are limited by not considering moisture dependent conductivity as well, in contrast to the results presented in section 5.4 which investigate the effective conductivity as a function of both temperature and humidity, though only for the unaged samples.

Additional future developments of this research should include further aging analysis, including full moisture testing on materials that have been aged through elevated temperature exposure. This will allow a fuller understanding of the effective conductivity after aging, allowing for simulations and calculations that may more accurately present an estimated performance of a building assembly farther along in its life cycle. 


\section{APPENDIX}

\section{WUFI Material Inputs}

Polyurethane A - Material Data
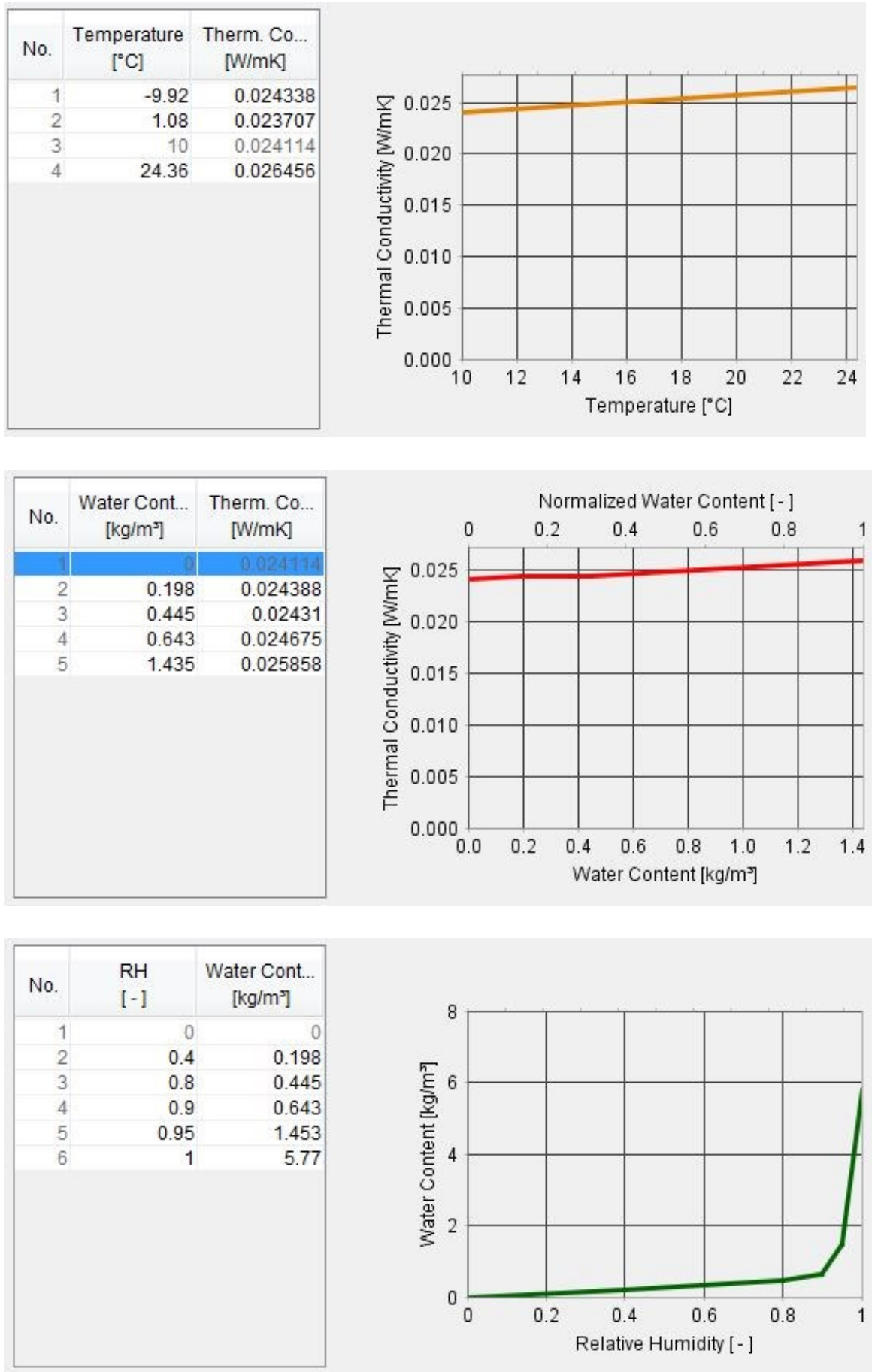
Polyurethane B - Material Data
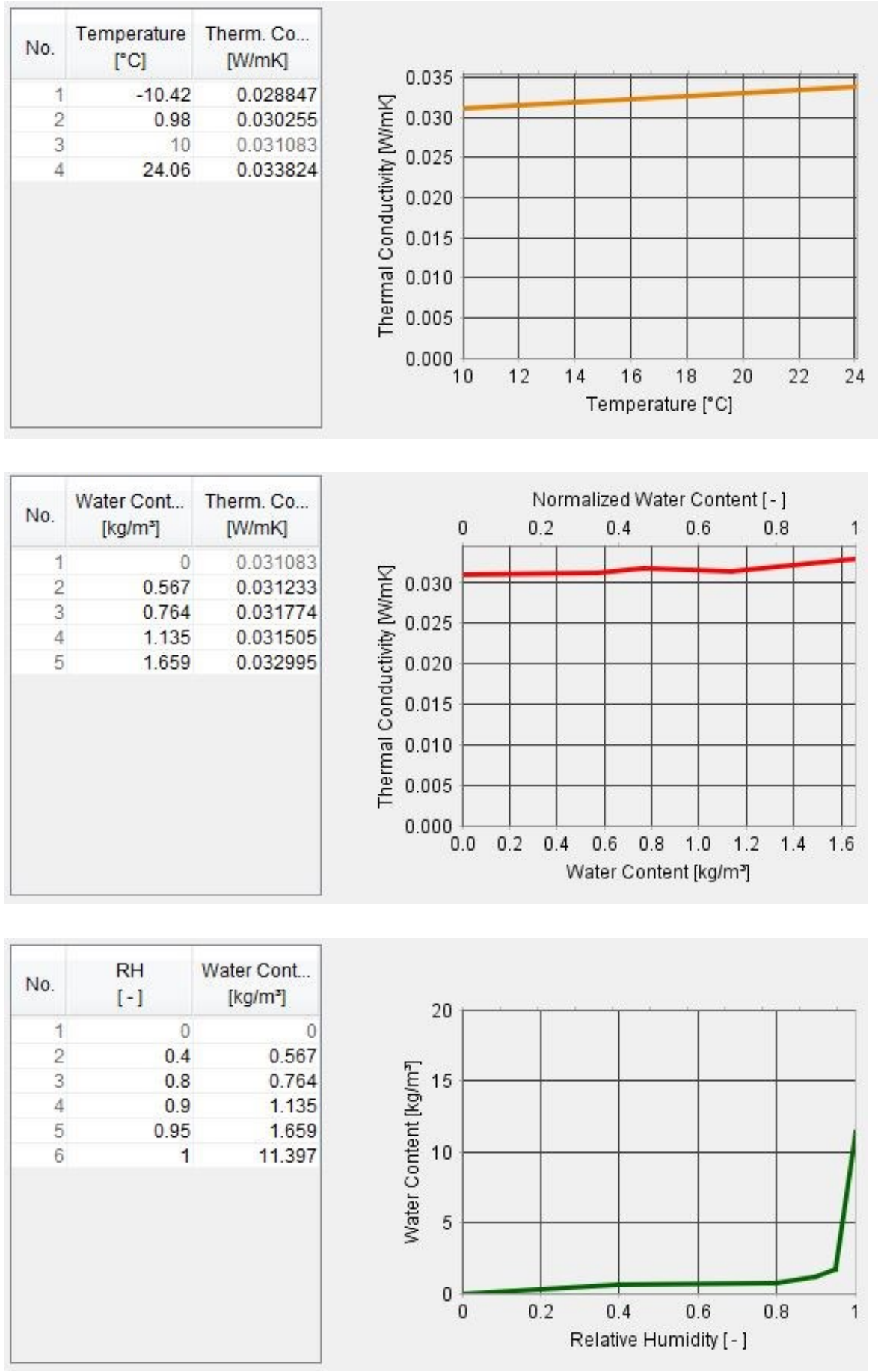
Polyurethane C - Material Data
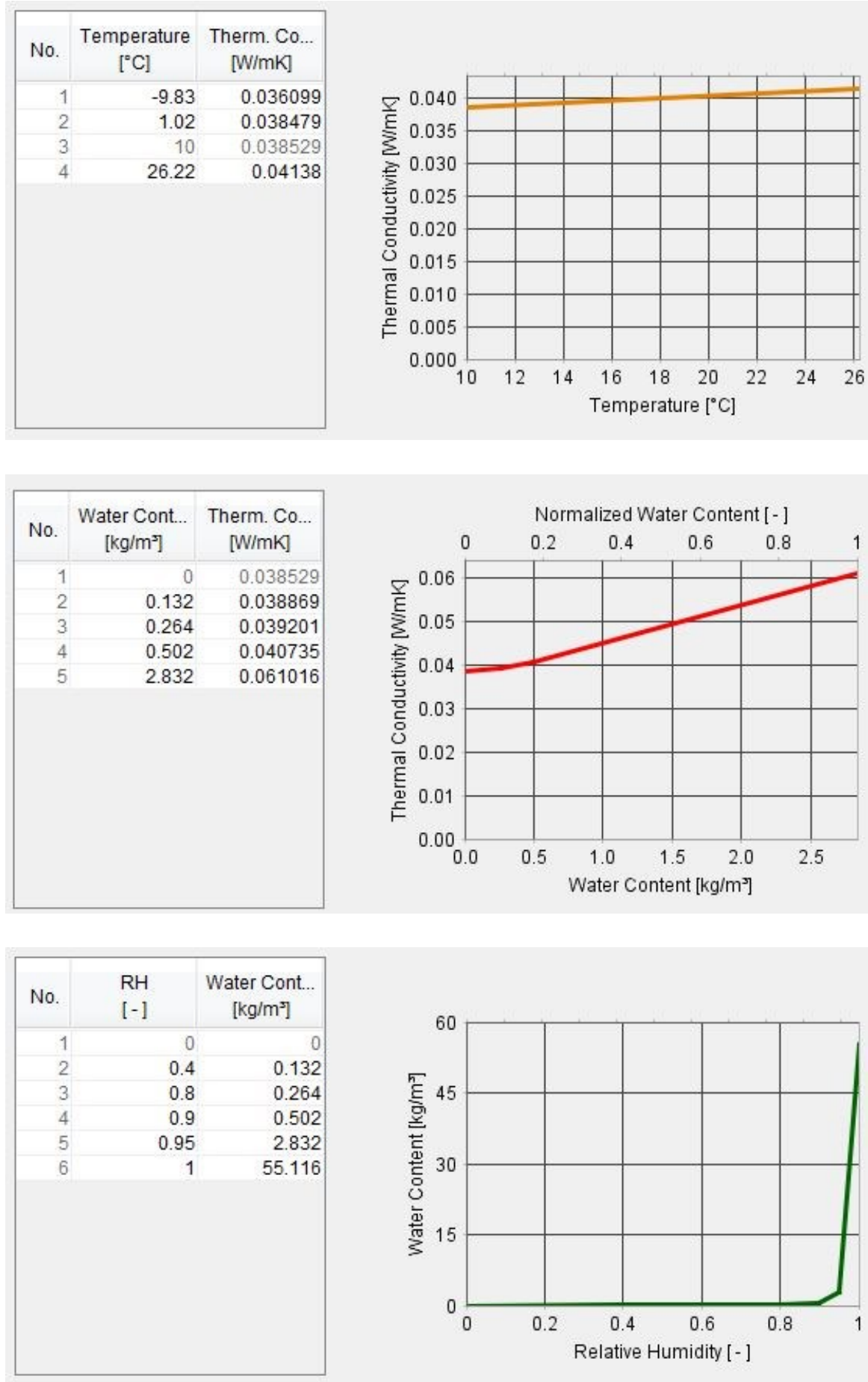


\section{Polyurethane D - Material Data}
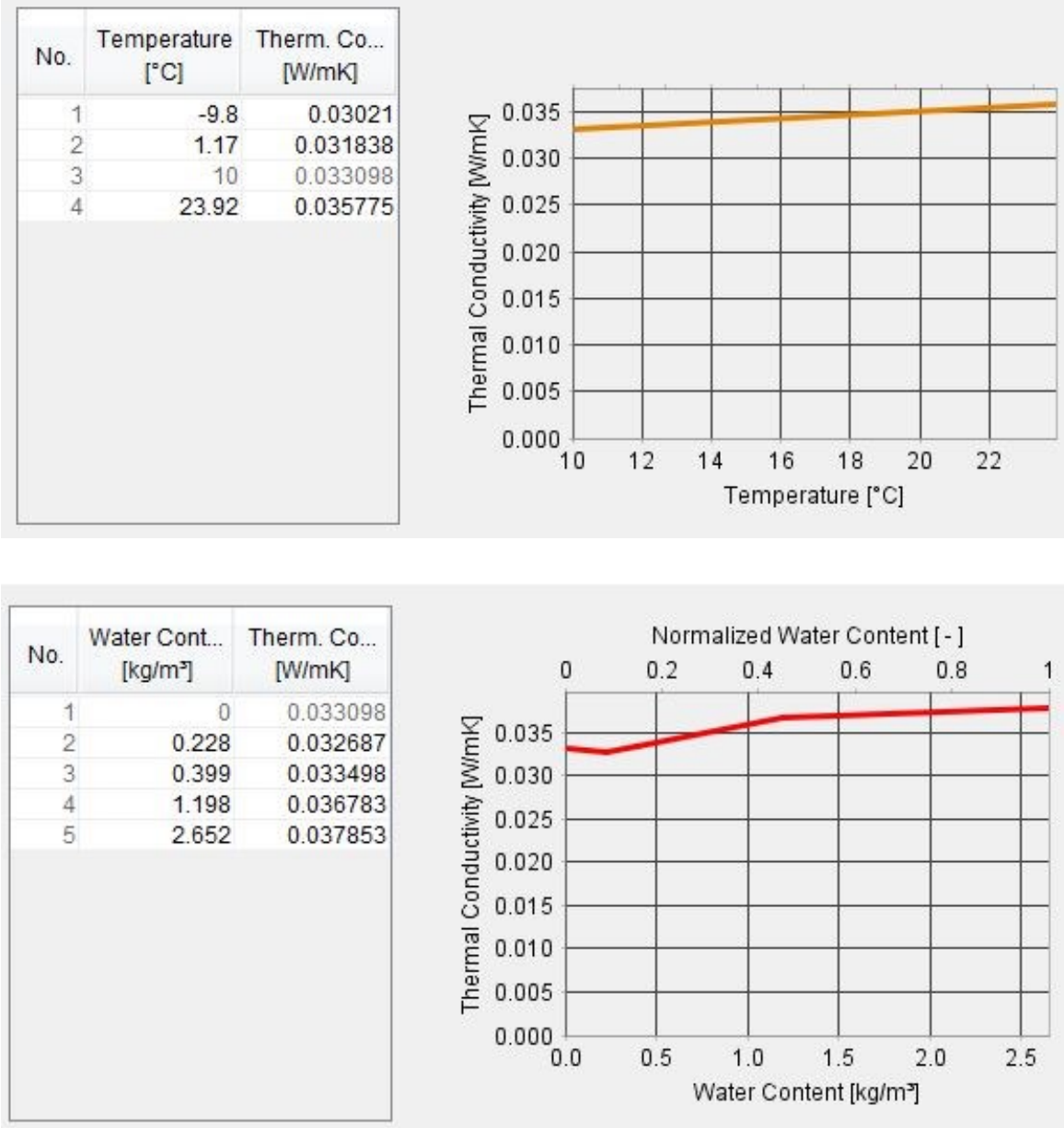

\begin{tabular}{|c|c|c|}
\hline No. & $\begin{array}{l}\mathrm{RH} \\
{[-]}\end{array}$ & $\begin{array}{c}\text { Water Cont... } \\
{\left[\mathrm{kg} / \mathrm{m}^{3}\right]}\end{array}$ \\
\hline 1 & 0 & 0 \\
\hline 2 & 0.4 & 0.228 \\
\hline 3 & 0.8 & 0.399 \\
\hline 4 & 0.9 & 1.198 \\
\hline 5 & 0.95 & 2.652 \\
\hline 6 & 1 & 30.435 \\
\hline
\end{tabular}

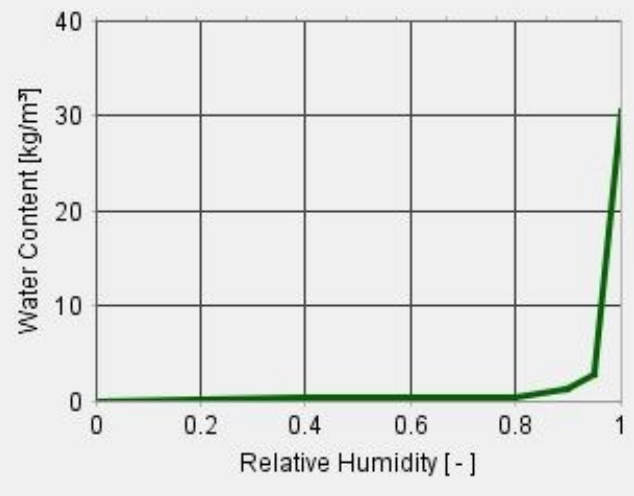




\section{Polyisocyanurate A - Material Data}
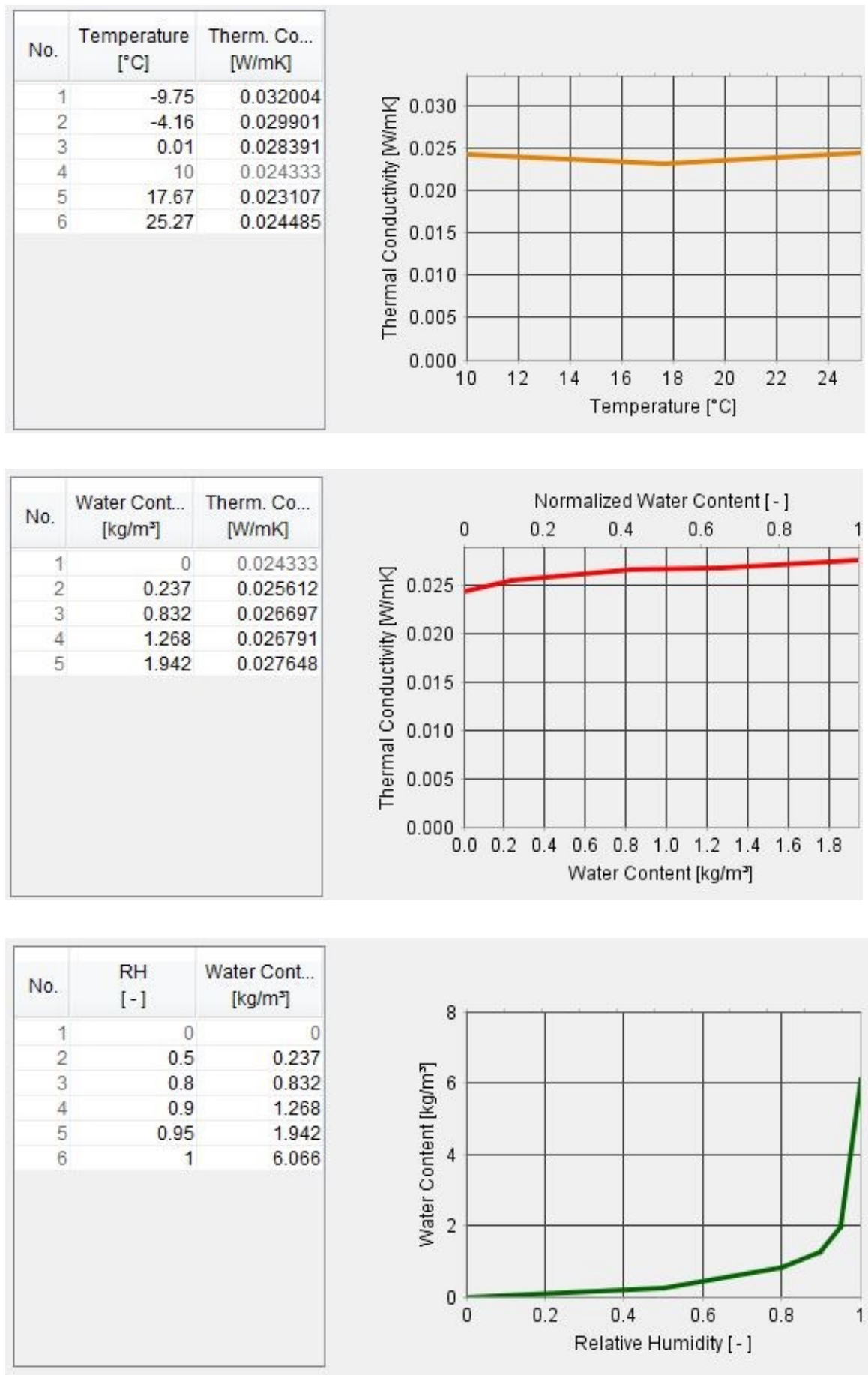
Polyisocyanurate B - Material Data
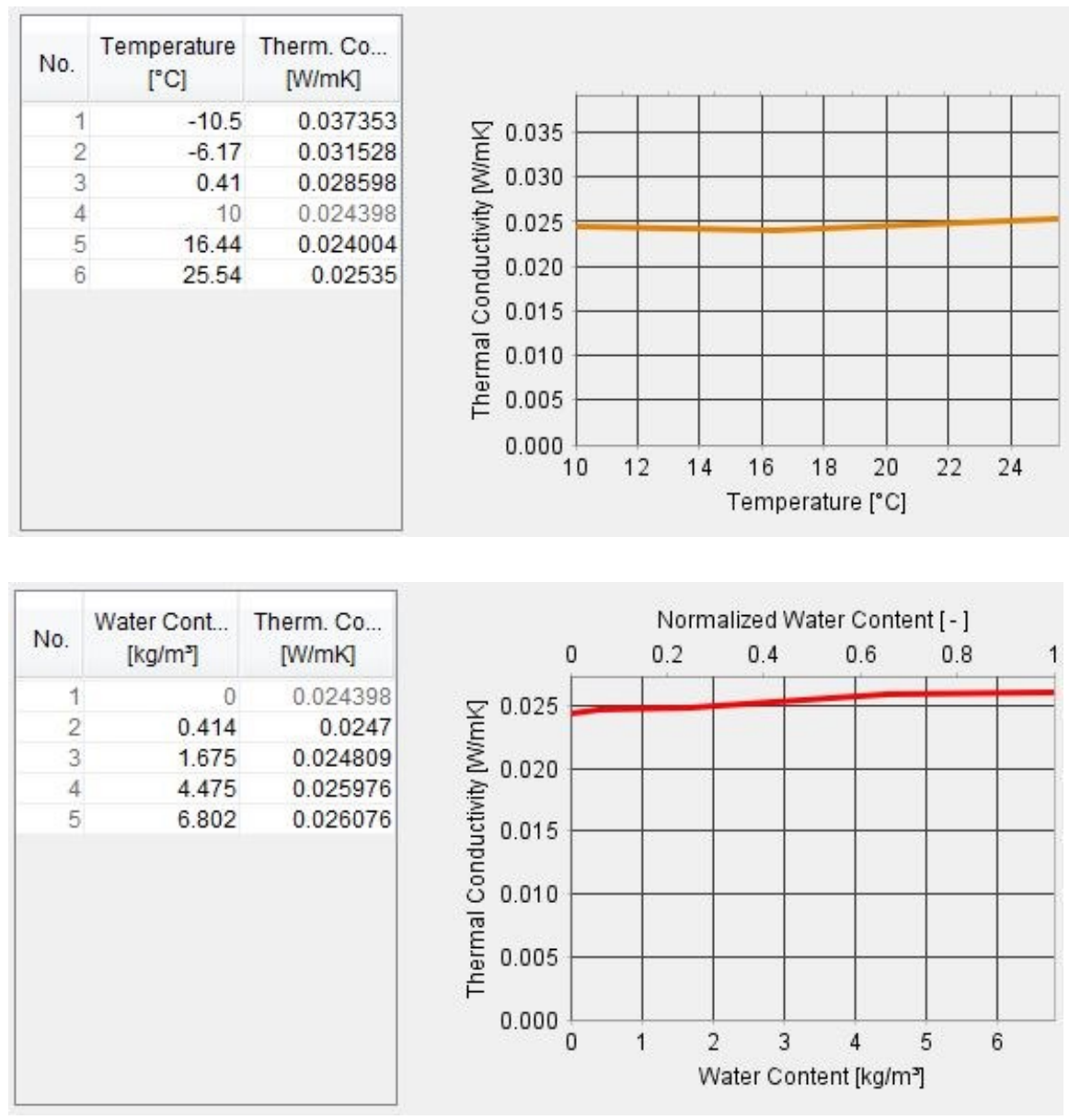

\begin{tabular}{|c|c|c|}
\hline No. & $\begin{array}{l}\mathrm{RH} \\
{[-]}\end{array}$ & $\begin{array}{c}\text { Water Cont... } \\
{\left[\mathrm{kg} / \mathrm{m}^{2}\right]}\end{array}$ \\
\hline 1 & 0 & 0 \\
\hline 2 & 0.5 & 0.414 \\
\hline 3 & 0.8 & 1.675 \\
\hline 4 & 0.9 & 4.475 \\
\hline 5 & 0.95 & 6.802 \\
\hline 6 & 1 & 10.094 \\
\hline
\end{tabular}

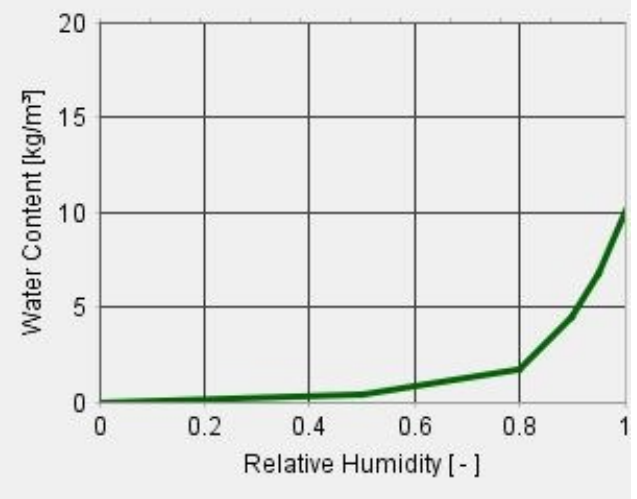




\subsection{3-D Surface Model Equations (MATLAB Outputs)}

The models developed here allow for the calculation of the effective conductivity given the temperature and humidity level. In the model equations, $\mathrm{x}$ is equal to the normalized temperature, and $\mathrm{y}$ is equal to the normalized relative humidity $\%$.

\section{PU-A - Formula Coefficients}

Linear model Poly23:

$$
\begin{aligned}
\operatorname{spf}(x, y) & =\text { p } 00+\text { p } 10 * x+p 01 * y+p 20 * x^{\wedge} 2+p 11 * x * y+p 02 * y^{\wedge} 2+p 21 * x^{\wedge} 2 * y \\
& + \text { p } 12 * x^{*} y^{\wedge} 2+p 03 * y^{\wedge} 3
\end{aligned}
$$

where $\mathrm{x}$ is normalized by mean 6.01 and std 13.14

and where $y$ is normalized by mean 63 and std 33.65

Coefficients (with $95 \%$ confidence bounds):

$$
\begin{aligned}
& \text { p00 }=0.02365(0.02309,0.0242) \\
& \text { p10 }=0.0006527(0.0002345,0.001071) \\
& \text { p01 }=-2.542 \mathrm{e}-05(-0.0007156,0.0006647) \\
& \text { p20 }=0.0006556(0.0003934,0.0009178) \\
& \text { p11 }=0.0003285(1.579 \mathrm{e}-05,0.0006411) \\
& \text { p02 }=0.001188(0.0004781,0.001898) \\
& \text { p21 }=-0.0001682(-0.0004398,0.0001033) \\
& \text { p12 }=0.0002362(-0.0001444,0.0006168) \\
& \text { p03 }=0.0007283(0.0001643,0.001292)
\end{aligned}
$$

err $=$

struct with fields:

sse: $1.9447 \mathrm{e}-06$

rsquare: 0.9372

dfe: 11

adjrsquare: 0.8916

rmse: $4.2047 \mathrm{e}-04$ 


\section{PU-B - Formula Coefficients}

\section{Linear model Poly23:}

$\operatorname{spf}(x, y)=\mathrm{p} 00+\mathrm{p} 10^{*} \mathrm{x}+\mathrm{p} 01 * \mathrm{y}+\mathrm{p} 20 * \mathrm{x}^{\wedge} 2+\mathrm{p} 11 * \mathrm{x}^{*} \mathrm{y}+\mathrm{p} 02 * \mathrm{y}^{\wedge} 2+\mathrm{p} 21 * \mathrm{x}^{\wedge} 2 * \mathrm{y}$

$$
+\mathrm{p} 12 * \mathrm{x}^{*} \mathrm{y}^{\wedge} 2+\mathrm{p} 03 * \mathrm{y}^{\wedge} 3
$$

where $\mathrm{x}$ is normalized by mean 6.039 and std 13

and where $y$ is normalized by mean 63 and std 33.65

Coefficients (with 95\% confidence bounds):

$$
\begin{aligned}
& \text { p00 }=0.03169(0.03102,0.03237) \\
& \text { p10 }=0.001762(0.001239,0.002285) \\
& \text { p01 }=0.001131 \quad(0.000282,0.00198) \\
& \text { p20 }=0.0002407 \quad(-7.707 \mathrm{e}-05,0.0005584) \\
& \text { p11 }=-0.0001094(-0.0004942,0.0002754) \\
& \text { p02 }=-0.0009289(-0.001814,-4.345 \mathrm{e}-05) \\
& \text { p21 }=-7.467 \mathrm{e}-05(-0.0004011,0.0002518) \\
& \text { p12 }=-5.579 \mathrm{e}-05(-0.0005242,0.0004126) \\
& \text { p03 }=-0.0007815(-0.001485,-7.816 \mathrm{e}-05)
\end{aligned}
$$

err $=$

struct with fields:

sse: $3.0316 \mathrm{e}-06$

rsquare: 0.9539

dfe: 11

adjrsquare: 0.9204

rmse: $5.2497 \mathrm{e}-04$ 


\section{PU-C - Formula Coefficients}

Linear model Poly23:

$\operatorname{spf}(x, y)=\mathrm{p} 00+\mathrm{p} 10^{*} \mathrm{x}+\mathrm{p} 01 * \mathrm{y}+\mathrm{p} 20^{*} \mathrm{x}^{\wedge} 2+\mathrm{p} 11^{*} \mathrm{x}^{*} \mathrm{y}+\mathrm{p} 02 * \mathrm{y}^{\wedge} 2+\mathrm{p} 21^{*} \mathrm{x}^{\wedge} 2^{*} \mathrm{y}$ $+\mathrm{p} 12 * \mathrm{x}^{*} \mathrm{y}^{\wedge} 2+\mathrm{p} 03 * \mathrm{y}^{\wedge} 3$

where $\mathrm{x}$ is normalized by mean 6.285 and std 13.06

and where $y$ is normalized by mean 63 and std 33.65

Coefficients (with 95\% confidence bounds):

$$
\begin{aligned}
& \text { p00 }=0.0312(0.01855,0.04385) \\
& \text { p10 }=0.0008619(-0.009102,0.01083) \\
& \text { p01 }=-0.003712(-0.01975,0.01233) \\
& \text { p20 }=-1.766 \mathrm{e}-05(-0.006246,0.00621) \\
& \text { p11 }=0.007204(-9.271 \mathrm{e}-05,0.0145) \\
& \text { p02 }=0.02069(0.003994,0.03738) \\
& \text { p21 }=0.0001139(-0.00598,0.006208) \\
& \text { p12 }=0.005488(-0.003264,0.01424) \\
& \text { p03 }=0.01276(-0.000507,0.02602)
\end{aligned}
$$

err $=$

struct with fields:

sse: 0.0011

rsquare: 0.6787

dfe: 11

adjrsquare: 0.4451

rmse: 0.0099 


\section{PU-D - Formula Coefficients}

Linear model Poly23:

$\operatorname{spf}(x, y)=\mathrm{p} 00+\mathrm{p} 10^{*} \mathrm{x}+\mathrm{p} 01 * \mathrm{y}+\mathrm{p} 20^{*} \mathrm{x}^{\wedge} 2+\mathrm{p} 11^{*} \mathrm{x}^{*} \mathrm{y}+\mathrm{p} 02 * \mathrm{y}^{\wedge} 2+\mathrm{p} 21^{*} \mathrm{x}^{\wedge} 2^{*} \mathrm{y}$

$$
+\mathrm{p} 12 * \mathrm{x}^{*} \mathrm{y}^{\wedge} 2+\mathrm{p} 03 * \mathrm{y}^{\wedge} 3
$$

where $\mathrm{x}$ is normalized by mean 6.133 and std 12.88

and where $y$ is normalized by mean 63 and std 33.65

Coefficients (with 95\% confidence bounds):

$$
\begin{aligned}
& \text { p00 }=0.03154(0.02875,0.03433) \\
& \text { p10 }=0.001511(-0.0006117,0.003634) \\
& \text { p01 }=0.001194(-0.002304,0.004692) \\
& \text { p20 }=0.0007243(-0.0005909,0.002039) \\
& \text { p11 }=0.002135(0.0005583,0.003711) \\
& \text { p02 }=0.00437(0.0007581,0.007981) \\
& \text { p21 }=0.000377(-0.0009887,0.001743) \\
& \text { p12 }=0.001771(-0.0001545,0.003697) \\
& \text { p03 }=0.002022(-0.000848,0.004892)
\end{aligned}
$$

err $=$

struct with fields:

sse: $5.0383 \mathrm{e}-05$

rsquare: 0.8846

dfe: 11

adjrsquare: 0.8007

rmse: 0.0021 


\section{PI-A - Formula Coefficients}

Linear model Poly23:

$\operatorname{spf}(x, y)=p 00+$ p $10 * x+p 01 * y+p 20 * x^{\wedge} 2+$ p $11 * x^{*} y+p 02 * y^{\wedge} 2+p 21 * x^{\wedge} 2 * y$ $+\mathrm{p} 12 * \mathrm{x}^{*} \mathrm{y}^{\wedge} 2+\mathrm{p} 03 * \mathrm{y}^{\wedge} 3$

where $\mathrm{x}$ is normalized by mean 5.981 and std 13.03

and where $y$ is normalized by mean 63 and std 33.36

Coefficients (with 95\% confidence bounds):

$$
\begin{aligned}
& \text { p00 }=0.02947(0.02735,0.03159) \\
& \text { p10 }=-0.005588(-0.007105,-0.004071) \\
& \text { p01 }=0.00582(0.00315,0.008489) \\
& \text { p20 }=0.002495(0.001411,0.003579) \\
& \text { p11 }=-0.003298(-0.004416,-0.00218) \\
& \text { p02 }=-0.0001285(-0.002751,0.002494) \\
& \text { p21 }=0.0006449(-0.0004787,0.001769) \\
& \text { p12 }=-0.0012(-0.0026,0.0002005) \\
& \text { p03 }=-0.001479(-0.00356,0.0006021)
\end{aligned}
$$

err $=$

struct with fields:

sse: $8.8164 \mathrm{e}-05$

rsquare: 0.9545

dfe: 21

adjrsquare: 0.9372

rmse: 0.0020 


\section{PI-B - Formula Coefficients}

Linear model Poly23:

$\operatorname{spf}(\mathrm{x}, \mathrm{y})=\mathrm{p} 00+\mathrm{p} 10^{*} \mathrm{x}+\mathrm{p} 01 * \mathrm{y}+\mathrm{p} 20^{*} \mathrm{x}^{\wedge} 2+\mathrm{p} 11^{*} \mathrm{x}^{*} \mathrm{y}+\mathrm{p} 02^{*} \mathrm{y}^{\wedge} 2+\mathrm{p} 21^{*} \mathrm{x}^{\wedge} 2 * \mathrm{y}$ $+\mathrm{p} 12 * \mathrm{x}^{*} \mathrm{y}^{\wedge} 2+\mathrm{p} 03 * \mathrm{y}^{\wedge} 3$

where $\mathrm{x}$ is normalized by mean 4.002 and std 12.54

and where $y$ is normalized by mean 63 and std 33.48

Coefficients (with $95 \%$ confidence bounds):

$$
\begin{aligned}
& \text { p00 }=0.02936(0.02702,0.0317) \\
& \text { p10 }=-0.009105(-0.01107,-0.007141) \\
& \text { p01 }=0.002745(-0.0001665,0.005656) \\
& \text { p20 }=0.004121 \quad(0.002925,0.005318) \\
& \text { p11 }=-0.002872(-0.004329,-0.001415) \\
& \text { p02 }=0.001298(-0.001732,0.004327) \\
& \text { p21 }=0.000803(-0.00035,0.001956) \\
& \text { p12 }=-0.0004008(-0.002005,0.001203) \\
& \text { p03 }=0.0004769(-0.001916,0.00287)
\end{aligned}
$$

err $=$

struct with fields:

sse: $7.0744 \mathrm{e}-05$

rsquare: 0.9639

dfe: 16

adjrsquare: 0.9459

rmse: 0.0021 


\section{BIBLIOGRAPHY}

ASTM 518, Standard Test Method for Steady-State Thermal Transmission Properties by Means of the Heat Flow Meter Apparatus (2015).

ASTM C1058, Standard Practice for Selecting Temperatures for Evaluating and Reporting Thermal Properties of Thermal Insulation (2015).

ASTM C1303, Standard Test Method for Predicting Long-Term Thermal Resistance of Closed-Cell Foam Insulation (2012).

Abdou, A., Budaiwi, I. The impact of thermal conductivity change of moist fibrous insulation on energy performance of buildings under hot-humid conditions. Architectural Engineering Department, King Fahd University of Petroleum and Minerals (KFUPM), Dhahran, Saudi Arabia. 2013.

Abdou, A., Budaiwi, I. The variation of thermal conductivity of fibrous insulation materials under different levels of moisture content. King Fahd University of Petroleum and Minerals (KFUPM), Dhahran, Saudi Arabia. 2013.

Beentjes, Ivan. Wright, John. Thermal Conductivity Measurements of Building Insulation Using a Guarded Heater Plate Apparatus. University of Waterloo, Waterloo, ON. 2012.

Berardi U., Naldi M., The impact of the temperature dependent thermal conductivity of insulating materials on the effective building envelope performance, Energy and Building(2017) 262-275.

Berardi U., Nosrati R., Long-term behaviour of aerogel-enhanced insulating materials under different aging laboratory conditions, Energy, 147 (2018) 1188-1202.

Berardi U., The temperature dependency of the building insulation thermal conductivity in the Canadian climate, Energy Procedia, 132 (2017) 237-242.

Bhattacharjee, D., Irwin, P.W., Booth, J.R., Grimes, J.T., The Acceleration of Foam Aging by ThinSlicing: Some Interpretations and Limitations, Journal of Building Physics, 17(3) (1994) 219237.

Bogdan, M., Gittere, C.,Ross, M., Honeywell's next generation (LGWP) of blowing agents for global spray foam applications, Honeywell Technical Presentation, Center for the Polyurethanes Industry conference (2011).

CAN/ULC-S770-15. Standard test method for determination of long-term thermal resistance of closedcell thermal insulating foams (2015).

CUFCA, Use of LTTR in determining RSI of spray polyurethane foam, Technical Bulletin, Canadian Urethane Foam Contractors Association (2005)

Desjarlais, A., Kyle, D. Assessment of technologies for constructing self-drying low-slope roofs, Office of Scientific and Technical Information, U.S. Dept. of Energy (1994). 
Garber-Slaght R., Moisture Measurement in Polyurethane Foam Insulation, Cold Climate Research Center, Report for Alaska House Financing Corp. (2012).

Grin A., Schumacher C., Smegal J., The Implications of Temperature-Dependent Thermal Conductivity for Commercial Roof Systems. RDH Building Science Consulting Inc. (2014).

Government of Canada, Montreal Protocol on Substances that Deplete the Ozone Layer (Protocol to the Vienna Convention for the Protection of the Ozone Layer, Environment Canada (1987)

Hunter, L., White, J., Cohen, P., Biermann, P., A Materials Aging Problem in Theory and Practice. John Hopkins APL Technical Digest, Vol 21, No 4, (2000)

Jelle, Petter. Evaluation of Building Products by Conducting Accelerated Climate Ageing in the Laboratory, XII DBMC, Porto, PORTUGAL, (2011)

Khoukhi, M., Draoui, B., Fezzioui N., Salah, L. The impact of changes in thermal conductivity of polystyrene insulation material under different operating temperatures on the heat transfer through the building envelope. College of Engineering, Sultan Qaboos University, Muscat, Oman. 2016

Lepage R., Schumacher C., Straube J., Luxachko A., The implications of temperature dependent thermal conductivity of exterior wall using insulated sheathing, RDH Building Science Consulting Inc. (2013).

Ludwick A., Aglan H., Abdalla M., Calhoun M., Degradation Behavior of and Ultraviolet and Hygrothermally Aged Polyurethane Elastomer: Fourier Transform Infrared and Differential Scanning Calorimetry Studies, Journal of Applied Polymer Science, 110 (2008) 712-718.

Muller-Steinhagen, H., Ochs, F. Temperature and Moisture Dependence of the Thermal Conductivity of Insulation Materials. NATO Advanced Study Institute on Thermal Energy Storage for Sustainable Energy Consumption (TESSEC), Izmir, Cesme, (2005)

Ross L., A blowing agent update - Learn why the change to pentane was made and what it means for polyiso, NRCA Professional Roofing Magazine (2005).

Shukla, N., Reducing the Thermal Aging Effect in Polyisocyanurate Foams. Fraunhofer USA, (2017).

Stovall T., Closed Cell Foam Insulation: A Review of Long Term Thermal Performance Research, Oak Ridge National Laboratory, ORNL/TM-2012/583 (2012).

Tseng, C., Yamaguchi, M., Ohmori, T. Thermal Conductivity of Polyurethane foams from room temperature to 20K. The New Energy and Industrial Technology Development Organization. Tokyo, Japan. 1997.

Yarbrough, D. Apparent Thermal Conductivity of Polyisocyanurate Foam Board Insulation as a Function of Temperature - Supplementary Report. R\&D Services Inc. Cookeville, TN. 2014. 